Aus dem Institut für Agrikulturchemie

der Georg-August-Universität Göttingen

\title{
Phosphordüngewirkung von Klärschlämmen aus Klärwerken mit Phosphateliminierung durch Eisensalze
}

(Phosphate fertilization effects of sewage sludges from waste water processing plants with phosphate elimination by iron salts)

\author{
Dissertation \\ zur Erlangung des Doktorgrades \\ der Fakultät für Agrarwissenschaften \\ der Georg-August-Universität Göttingen
}

vorgelegt von

M. Sc. agr. Ihab Mohamed Farid Abd El-Samie geboren in Kairo, Ägypten

Göttingen, 6. Februar 2003

Gefördert durch die ägyptische Regierung,

das Niedersächsische Ministerium für Ernährung, Landwirtschaft und Forsten sowie die Niedersächsische Umweltstiftung 
Abd El-Samie, Ihab Mohamed Farid

Phosphordüngewirkung von Klärschlämmen aus Klärwerken mit Phosphateliminierung durch Eisensalze

Göttingen, Universität, Dissertation, 2003

D7

Referent:

Prof. Dr. W. Römer

Korreferent:

Prof. Dr. B. Meyer

Tag der mündlichen Prüfung:

6. Februar 2003 
1. Referent: $\quad$ Prof. Dr. W. Römer

2. Korreferent: Prof. Dr. B. Meyer

Tag der mündlichen Prüfung: 6.02.2003 


\section{WIDMUNG}

Diese Arbeit widme ich

meiner Familie

besonders im Gedenken

an meinen Vater

\section{DEDICATION}

To my late father,

to my mother,

to my brothers,

to my wife and children 


\section{Inhaltsverzeichnis}

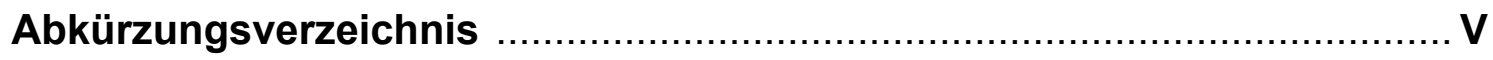

Abbildungsverzeichnis ……............................................................. VII

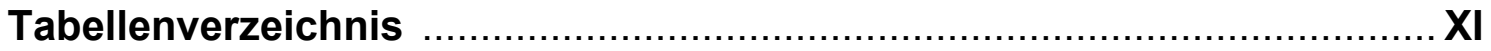

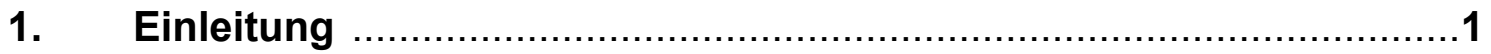

\section{Teil I}

2. Mehrjährige Experimente zur P-Aufnahme von Kulturpflanzen und zur P- Verfügbarkeit in Böden nach Applikation von zwei

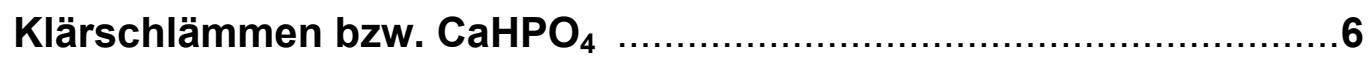

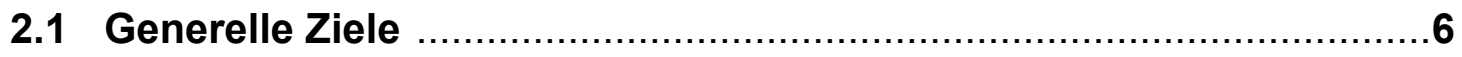

2.2 Dreijähriges Experiment zur P - Aufnahme der Pflanzen …............6

2.2.1 Vorbemerkungen und Versuchsziele ……...............................6

2.2.2 Versuchsanlage und Durchführung ………….........................

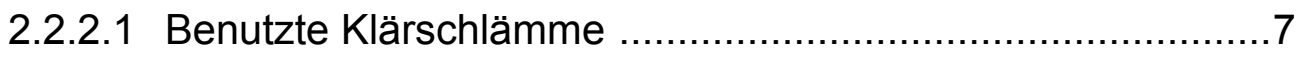

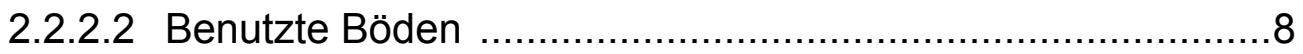

2.2.2.3 Anlage des Gefäßversuchs zur P-Aufnahme ....................

1. Versuchsjahr 1999 (Mais) ...............................................

2. Versuchsjahr 2000 (Rotklee) ………………….........11

3. Versuchsjahr 2001 (Weidelgras) …...............................12 
2.2.2.4 Pflanzenanalyse auf Phosphor

Gefäßversuche mit Mais

Maisanbau auf Boden mit hohem P-Gehalt

Maisanbau bei niedrigem und mäßigem P- Gehalt der Böden bzw. Substrate

Gefäßversuch mit Rotklee 19

Gefäßversuch mit Weidelgras

2.2.2.6 Überblick über die $P$ - Entzüge in den drei Versuchsjahren .23

2.3 Zweijähriges Experiment zur Prüfung der P- Aufnahme der Pflanzen

2.3.1 Vorbemerkungen und Versuchsziele 25

2.3.2 Versuchsanlage und Durchführung .25

2.3.3 Ergebnisse: Sprossmassebildung und P- Entzüge .28

\subsection{Einjähriges Experiment zur Verfolgung der P- Verfügbarkeit in} neun Böden nach Klärschlammdüngung

2.4.1 Vorbemerkungen und Versuchsziele .33

2.4.2 Versuchsanlage und Durchführung .34

2.4.3 Analysenmethoden in Böden .35 


\section{III}

2.4.4 Ergebnisse des Inkubationsversuches zu Verfolgung der PVerfügbarkeit über 12 Monate

2.4.4.1 Orthophosphatkonzentration $\left(\mathrm{P}_{\mathrm{i}}\right)$ in den Bodenlösungen ....37

2.4.4.2 P-Sorption der Böden .40

2.4.4.3 Beziehung zwischen den Bodengehalten an $\mathrm{Fe}_{\mathrm{ox}}$ und der P- Sorption

2.5 Diskussion und Schlussfolgerungen .47

Teil II

3. Einjährige Experimente zur P- Aufnahme von Kulturpflanzen und P- Verfügbarkeit im Boden nach Applikation von 13 Klärschlämmen und verschiedener $P$ - Dünger in einen humosen Sandboden

3.1 Vorbemerkungen und Versuchsziel

3.1.1 Verwendete Klärschlämme .58

3.1.2 Verwendeter Boden .59

3.1.3 Genereller Versuchsaufbau 60

3.1.3.1 Durchführung des Gefäßversuches mit Weidelgras unter Freilandbedingungen (Drahthaus)

3.1.4 Ergebnisse

3.1.4.1 Erträge und P- Entzüge des Grases 62

3.1.4.2 Bodenkenngrößen .69 


\begin{abstract}
IV
3.1.4.3 Beziehungen zwischen den P- Entzügen und Bodenkenngrößen bzw. zwischen verschiedenen Bodenkenngrößen (Regressionsanalysen)
\end{abstract} .72

3.1.5 Diskussion und Schlussfolgerungen .83

Teil III

4. Kleingefäßversuch zur Prüfung der P- Aufnahme aus drei Klärschlämmen mit unterschiedlichen Werten des molaren Fe:

P- Verhältnisses auf zwei verschiedenen ägyptischen Böden .87

4.1 Vorbemerkungen und Versuchsziele 87

4.2 Versuchsanlage und Durchführung .87

4.3 Ergebnisse .90

5. Abschließende Bemerkungen .93

6. Zusammenfassung .94

7. Literaturverzeichnis 100

8. Anhang .104

Danksagung

Lebenslauf

Publikationen

Englische Zusammenfassung (Three Abstracts) 


\section{V}

\section{Abkürzungsverzeichnis}

AAS

AbfKlärV

ATV

CAL- $P$

Ca-P- Variante

DL- P

EU

EWG

$\mathrm{Fe}_{\mathrm{ox}}$

GD

GK

Hy

ICP

KO

KS Gö

KS Sh

KS

Mill

Mol. Fe : P

P- Sorp

$\mathrm{P}$

$\mathrm{P}_{\mathrm{i}}-$ Konz

$\mathrm{P}_{\text {ox }}$

Qs
Atomabsorptionsspektrometer

Klärschlammverordnung

Abwassertechnische Vereinigung

Calciumacetatlactat- P

$\mathrm{CaHPO}_{4}$ - Variante

Doppellactat- $\mathrm{P}$

Europäische Union

Europäische Wirtschaftsgemeinschaft

oxalatlösliches Eisen

Grenzdifferenz

Gehaltsklasse

Hyperphosphat

Induced Coupled Plasma

Kontrolle

Klärschlamm Göttingen

Klärschlamm Salzhausen

Klärschlamm, Klärschlämme

Million

molares Fe : P- Verhältnis

Phosphat-Sorption

Phosphat, Phosphor

Orthophosphat-Konzentration

oxalatlösliches Phosphat

Quarzsand 


\begin{tabular}{ll} 
& \multicolumn{1}{c}{ VI } \\
$r$ & Korrelationskoeffizient \\
$r^{2}$ & Bestimmtheitsmaß \\
TM & Trockenmasse \\
TSP & Triplesuperphosphat \\
Tukey 0,05 & signifikante Unterschiede nach Durchführung eines \\
& Tukeytestes bei $5 \%$ Irrtumswahrscheinlichkeit \\
VDLUFA & Verband Deutscher Landwirtschaftlicher Untersuchungs- \\
& und Forschungsanstalten \\
WG & Weidelgras \\
WK & Wasserkapazität
\end{tabular}




\section{VII}

\section{Abbildungsverzeichnis}

Abbildung 1: Abhängigkeit des Maisertrages und des P- Entzuges von der P- Applikation zum Boden Börry P-80 ............................14

Abbildung 2: Abhängigkeit des Maisertrages und des P- Entzuges von der P- Applikation zum Quarzsand 15

Abbildung 3: Abhängigkeit des Maisertrages und des P- Entzuges von der P- Applikation zum Boden Elliehausen

Abbildung 4: Abhängigkeit des Maisertrages und des P- Entzuges von der P- Applikation zum Boden Rehburg-Loccum

Abbildung 5: Abhängigkeit des Maisertrages und des P- Entzuges von der P- Applikation zum Boden Düshorn (Brache)

Abbildung 6: Abhängigkeit des Maisertrages und des P- Entzuges von der P- Applikation zum Boden Börry P-0 19

Abbildung 7: Beziehungen zwischen den Bodengehalten an oxalatlöslichem Eisen (Feox) und der P- Sorption in zwei P- Varianten eines Schluffbodens (Börry P-0, P-80) 1 Monat nach der KS- Applikation

Abbildung 8: Beziehungen zwischen den Bodengehalten an oxalatlöslichem Eisen (Feox) und der P- Sorption in zwei P- Varianten eines Lehmbodens (Dorstadt P-0, P-20) ein Monat nach der KS- Applikation

Abbildung 9: Beziehungen zwischen den Bodengehalten an oxalatlöslichem Eisen (Feox) und der P- Sorption in drei Sandböden (Düshorn (Brache), P-22; Hodenhagen P-22) ein Monat nach der KS- Applikation

Abbildung 10: Abhängigkeit des P- Entzuges des Weidelgrases vom DL-P-Gehalt des Bodens nach KS- Applikation

Abbildung 11: Abhängigkeit des P- Entzuges des Weidelgrases von der

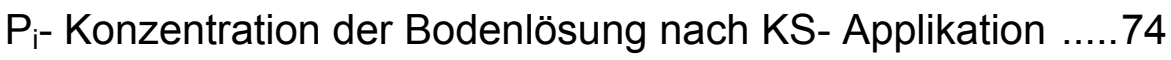




\section{VIII}

Abbildung 12: Abhängigkeit des P- Entzuges des Weidelgrases von der

P-Sorption des Bodens nach KS- Applikation .75

Abbildung 13: Abhängigkeit des P- Entzuges des Weidelgrases vom $\mathrm{Fe}_{\text {ox}}-$ Gehalt des Bodens nach KS- Applikation .75

Abbildung 14: Abhängigkeit des P- Entzuges des Weidelgrases vom $\mathrm{Fe}_{o x}-$ Gehalt des Bodens nach KS- Applikation .76

Abbildung 15: Abhängigkeit des P- Entzuges des Weidelgrases vom molaren Fe: P- Verhältnis der Klärschlämme

Abbildung 16: Abhängigkeit der Pi- Konzentration vom DL-P-Gehalt des Bodens nach KS- Applikation

Abbildung 17: Abhängigkeit der $\mathrm{P}_{\mathrm{i}}$ - Konzentration von der $\mathrm{P}$ - Sorption des Bodens nach KS- Applikation

Abbildung 18: Abhängigkeit der $\mathrm{P}_{\mathrm{i}}$ - Konzentration vom Bodengehalt an $\mathrm{Fe}_{\mathrm{ox}}$ nach der KS- Applikation 80

Abbildung 19: Abhängigkeit der $\mathrm{P}_{\mathrm{i}}-$ Konzentration in der Bodenlösung vom Boden- $\mathrm{pH}$ nach der KS- Applikation

Abbildung 20: Abhängigkeit der $\mathrm{P}_{\mathrm{i}}-$ Konzentration vom molaren $\mathrm{Fe}$ : $\mathrm{P}-$ Verhältnis der Klärschlämme

Abbildung 21: Abhängigkeit der $\mathrm{P}$ - Sorption vom $\mathrm{Fe}_{\mathrm{ox}}-$ Gehalt des Bodens nach der KS- Applikation

Abbildung 22: Abhängigkeit der P-Sorption vom Boden- $\mathrm{pH}$ nach der KS- Applikation

Abbildung 23: Abhängigkeit der P- Sorption vom molaren Fe: P- Verhältnis der Klärschlämme

Abbildung 24: Abhängigkeit des Maisertrages und des P- Entzuges von der P- Applikation zum Lehmboden Moschtohor .90

Abbildung 25: Abhängigkeit des Maisertrages und des P- Entzuges von der P- Applikation zum Sandboden Meetkenana 


\section{IX}

Abbildung 8.1: Abhängigkeit des Rotkleeertrages und des P- Entzuges von der P- Applikation zum Quarzsand

Abbildung 8.2: Abhängigkeit des Rotkleeertrages und des P- Entzuges von der P-Applikation zum Boden Elliehausen 120

Abbildung 8.3: Abhängigkeit des Rotkleeertrages und des P- Entzuges von der P-Applikation zum Boden Rehburg-Loccum

Abbildung 8.4: Abhängigkeit des Rotkleeertrages und des P- Entzuges von der P-Applikation zum Boden Düshorn (Brache)

Abbildung 8.5: Abhängigkeit des Rotkleeertrages und des P- Entzuges von der P-Applikation zum Boden Börry P-0

Abbildung 8.6: Abhängigkeit des Rotkleeertrages und des P- Entzuges von der P-Applikation zum Boden Börry P-80

Abbildung 8.7: Abhängigkeit des Weidelgrasertrages und des PEntzuges von der P- Applikation zum Quarzsand 125

Abbildung 8.8: Abhängigkeit des Weidelgrasertrages und des PEntzuges von der P- Applikation zum Boden Elliehausen ...126

Abbildung 8.9: Abhängigkeit des Weidelgrasertrages und des PEntzuges von der P- Applikation zum Boden RehburgLoccum

Abbildung 8.10: Abhängigkeit des Weidelgrasertrages und des PEntzuges von der P- Applikation zum Boden Düshorn (Brache) 128

Abbildung 8.11: Abhängigkeit des Weidelgrasertrages und des PEntzuges von der P- Applikation zum Boden Börry P-0 ......129

Abbildung 8.12: Abhängigkeit des Weidelgrasertrages und des PEntzuges von der P- Applikation zum Boden Börry P-80 ....130 


\section{$\mathbf{X}$}

\section{Tabellenverzeichnis}

Tabelle 1: Kenndaten der gefriergetrockneten applizierten

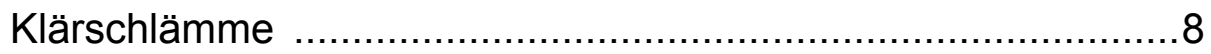

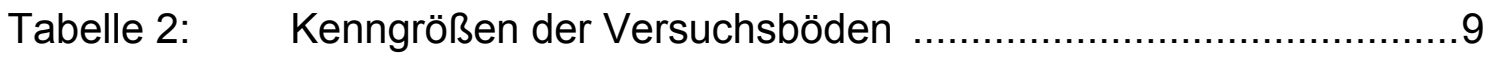

Tabelle 3: P- Düngungsvarianten des dreijährigen Experimentes ...........10

Tabelle 4: $\quad$ Relativerträge der Rotkleetrockenmasse (Summe von 3 Schnitten) nach der P-Düngung in Höhe von $187 \mathrm{mg} \mathrm{P} \mathrm{je}$ Gefäß zum Mais (Vorfrucht) ...... .20

Tabelle 5: $\quad$ Relative P-Entzüge der Rotkleepflanzen (Summe von 3

Schnitten) als 2. Kultur nach einer P-Düngung von $187 \mathrm{mg}$

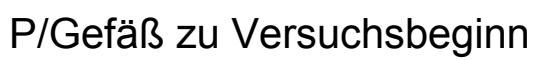
.20

Tabelle 6: $\quad$ Relativerträge der Weidelgrastrockenmasse (Summe von 3 Schnitten) nach der P-Düngung in Höhe von $187 \mathrm{mg} \mathrm{P} \mathrm{je}$ Gefäß zu Versuchsbeginn 21

Tabelle 7: $\quad$ Relative P-Entzüge der Weidelgraspflanzen (Summe von 3 Schnitten) als 3. Kultur nach einer P-Düngung von $187 \mathrm{mg}$

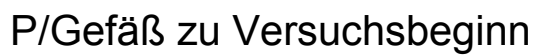
22

Tabelle 8: $\quad$ Übersicht über die relativen P-Entzüge nach

Klärschlammdüngung mit einer $\mathrm{P}$ - Gabe von $187 \mathrm{mg} \mathrm{P}$

Gefäß durch Mais, Rotklee und Weidelgras (WG) auf verschiedenen Böden $\left(\mathrm{P}\right.$-Entzug nach $\mathrm{CaHPO}_{4}$-Düngung $=$ $100 \%)$ 24

Tabelle 9: Kenndaten der verwendeten Böden (Korngrößenanteile, $\mathrm{CaCO}_{3}-$ Gehalt und Humusgehalt nach Angaben der LUFA Hameln) 26

Tabelle 10: Versuchsaufbau des zweijährigen Experimentes .27

Tabelle 11: Absolut- und Relativerträge der Gesamttrockenmasse je Gefäß des Weidelgrases (3 Schnitte) .29 
Tabelle 12: Absolute und relative P-Entzüge je Gefäß des

Weidelgrases (3 Schnitte)

Tabelle 13: Einfluss einer Kalkung $(0,72 \mathrm{~g} \mathrm{CaO} / \mathrm{kg}$ Boden) auf den

Ertrag (Summe von 3 Schnitten) und P-Entzug von

Weidelgras ohne P-Düngung (Mittelwerte von 3

Wiederholungen)

32

Tabelle 14: Orthophosphatkonzentration $\left(P_{\mathrm{i}}\right)$ in der Bodenlösung $\left(\mu \mathrm{g} \mathrm{I}^{-1}\right)$

von 5 Schluffböden nach Inkubation von 1, 6 und 12

Monaten, P-Zugabe: $30 \mathrm{mg} \mathrm{P} / \mathrm{kg}$ Boden entsprechend 187

$\mathrm{mg} P$ / Gefäß

Tabelle 15: Orthophosphatkonzentration $\left(P_{\mathrm{i}}\right)$ in der Bodenlösung $\left(\mu \mathrm{g} \mathrm{I}^{-1}\right)$

von 4 Sandböden nach Inkubation von 1, 6 und 12

Monaten, P-Zugabe: 30mg P / kg Boden entsprechend 187

mg P / Gefäß

Tabelle 16: $\quad$ P-Sorption von 5 Schluffböden 1 Monat bzw. 6 und 12

Monate nach der P-Düngung, $30 \mathrm{mg} \mathrm{P} / \mathrm{kg}$ Boden

entsprechend $187 \mathrm{mg} P$ / Gefäß

Tabelle 17: $\quad$ P-Sorption von 4 Sandböden 1 Monat bzw. 6 und 12

Monate nach der P-Düngung, $30 \mathrm{mg} P$ / kg Boden

entsprechend $187 \mathrm{mg}$ P / Gefäß .43

Tabelle 18: Einfluss von Klärschlamm (Höhe der P-Gabe) und Inkubationszeit (1, 6 und 12 Monate) auf die relative P-

Sorption der Böden (P-Sorption der Böden ohne $P=100)$

Tabelle 19: $\quad$ pH-Werte der Böden 1 Monat nach der Düngung mit 187

mg P pro Gefäß (Mittelwerte aus 4 Wiederholungen)

Tabelle 20: Wichtige Kenndaten der gefriergetrockneten 13

Klärschlämme .59

Tabelle 21: Kenngrößen des Versuchsbodens Düshorn (Brache) .60 


\section{XII}

Tabelle 22: Versuchsvarianten der Gefäßversuche zur Prüfung von 13 Klärschlämmen auf ihre $P$ - Düngewirksamkeit ( $P$ Applikation: $30 \mathrm{mg} \mathrm{P} / \mathrm{kg}$ Boden) 61

Tabelle 23: Trockenmasseertrag und P-Entzug des Weidelgrases in Abhängigkeit von der mineralischen P-Quelle (1. bis 3. Schnitt) sowie die Relativwerte bezogen auf die Kontrolle (K0) ohne P-Düngung

Tabelle 24: Trockenmasseerträge des Weidelgrases in Abhängigkeit von der P-Quelle (1. bis 3. Schnitt) sowie die Relativerträge bezogen auf die Kontrolle (KO) ohne P-Düngung 65

Tabelle 25: P-Entzüge des Weidelgrases in Abhängigkeit von den PQuellen (1. bis 3. Schnitt) sowie die Relativwerte bezogen auf die Kontrolle (KO) ohne P-Düngung 66

Tabelle 26: Relative P-Entzüge aus den 13 Klärschlämmen bezogen auf die P- Entzüge aus TSP (3 Gruppen) (P-Entzug aus Triplesuperphosphat $=100 \%$ ) .66

Tabelle 27: TM-Ertrag und P-Entzug von Weidelgras nach Applikation von $\mathrm{Fe}(\mathrm{OH})_{3}($ Variante $\mathrm{Fe} 1, \mathrm{Fe} 2), \mathrm{Ca}(\mathrm{OH})_{2}(\mathrm{Ca} 1, \mathrm{Ca} 2)$ bzw. $\mathrm{Fe}(\mathrm{OH})_{3} / \mathrm{Ca}(\mathrm{OH})_{2}($ Variante $\mathrm{Fe} / \mathrm{Ca}) ;$ Kontrolle $=100 \% \ldots .68$

Tabelle 28: Einfluss der P- Mineral- und Klärschlammdüngung (KS) auf den DL-Phosphatgehalt, die $\mathrm{P}_{\mathrm{i}}$ - Konzentration der Bodenlösung und die $\mathrm{pH}-$ Werte des humosen Sandbodens 30 Tage nach der Düngerapplikation .70

Tabelle 29: Einfluss der P- Mineral- und Klärschlammdüngung (KS) auf die P-Sorption, die Bodengehalte an oxalatlöslichem Fe ( Feox ) und Phosphor ( Pox ) des humosen Sandbodens 30 Tage nach der Düngerapplikation

Tabelle 30: Bestimmtheitsmaße für 6 lineare Regressionen 73

Tabelle 31: Bestimmtheitsmaße zwischen verschiedenen Parametern des Bodens bzw. der Klärschlämme .78 


\section{XIII}

Tabelle 32: Kenndaten der gefriergetrockneten, applizierten

Klärschlämme

Tabelle 33: Kenngrößen der ägyptischen Versuchsböden .88

Tabelle 34: Versuchsaufbau und Design des Gefäßversuches in der Klimakammer .89

Tabelle 8.1: EU-Grenzwertvorschläge für organische Schadstoffe im Klärschlamm bei landwirtschaftlicher Verwertung a) 104

Tabelle 8.2: EU-Grenzwertvorschläge für Schwermetalle im Klärschlamm zur landwirtschaftlichen Verwertung a), (Grenzwerte können alternativ auf Trockenrückstand oder auf Phosphorgehalt bezogen werden)

Tabelle 8.3: Analysendaten der zwei gefriergetrockneten, applizierten

Klärschlämme 105

Tabelle 8.4: P-Sorption von 5 Lehmböden (absolut und relativ) 1, 6 und 12 Monate nach der P-Düngung 106

Tabelle 8.5: P-Sorption von 4 Sandböden (absolut und relativ) 1, 6 und 12 Monate nach der P-Düngung 107

Tabelle 8.6: $\quad P_{\mathrm{i}-}$ Konzentration $\left(\mathrm{mg} \mathrm{L}^{-1}\right)$ in der Bodenlösung 1 Monat, 6 und 12 Monate nach der P-Applikation zu den 5 Lehmböden 108

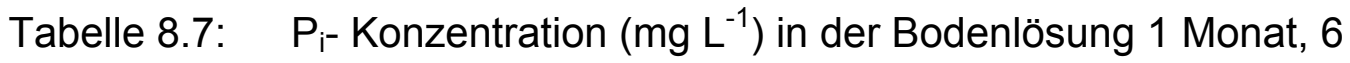
und 12 Monate nach der P-Applikation zu den 4 Sandböden 109

Tabelle 8.8: $\quad \mathrm{pH}$ - Werte der Lehmböden 1 Monat, 6 und 12 Monate nach der P-Applikation $\left(\mathrm{CaCl}_{2}\right.$ 0,01 M)

Tabelle 8.9: $\quad \mathrm{pH}$ - Werte der Sandböden 1 Monat, 6 und 12 Monate nach der P- Applikation $\left(\mathrm{CaCl}_{2}\right.$ 0,01 M)

Tabelle 8.10: P-Gehalte der Lehmböden (CAL- Extraktion) 1 Monat, 6 und 12 Monate nach der P-Applikation (mg P $100 \mathrm{~g}^{-1}$ ) 


\section{XIV}

Tabelle 8.11: P- Gehalte der Sandböden 1 Monat, 6 und 12 Monate nach der P- Applikation ( $\mathrm{mg} \mathrm{P}^{100 \mathrm{~g}^{-1}}$ )

Tabelle 8.12: Relativerträge an Maistrockenmasse je Gefäß

Tabelle 8.13: Relative P- Entzüge des Mais je Gefäß 115

Tabelle 8.14: Analysendaten zu den 13 Klärschlämmen (Gehalte in der TM), nach LUFA- Hameln 116

Tabelle 8.15: Mengen an gefriergetrocknetem Klärschlamm, die bei einer Applikation von ca. $30 \mathrm{mg} \mathrm{P} / \mathrm{kg}$ Boden in die Gefäße eingearbeitet wurden sowie die je $\mathrm{kg}$ Boden eingesetzten $\mathrm{Fe}-$ und $\mathrm{CaO}-$ Mengen

Tabelle 8.16: Absolute und relative Trockenmasseerträge und PEntzüge des Maises in Abhängigkeit von der P-Quelle auf dem Lehmboden Moschtohor 118

Tabelle 8.17: Absolute und relative Trockenmasseerträge und PEntzüge des Maises in Abhängigkeit von der P-Quelle auf dem Sandboden Meetkenana 
Gegenwärtig findet in der Europäischen Union (EU) und auch in Deutschland eine intensive Diskussion über die Verwertung von Klärschlämmen (KS) statt (Bannick, 2001, Hahn, 2001).

Ursache dafür ist, dass die EU- Klärschlammrichtlinie (86 / 278 / EWG) aus dem Jahr 1986 überarbeitet wird und dann die deutsche Klärschlammverordnung (Abf. Klär V) aus dem Jahr 1992 ebenfalls geändert werden muss. Bedeutsam an den geplanten Änderungen sind neue bzw. niedrigere Grenzwerte für organische Schadstoffe (Tabelle 8.1, Anhang) bzw. für Schwermetalle (Tabelle 8.2, Anhang) wie sie für die drei Etappen (2005, 2015, 2025) angestrebt werden (Bannick und Hahn, 2001).

Was diese neuen Richtlinien für die Verwertung der Klärschlämme in Deutschland bedeuten, ist noch unklar. In Deutschland fallen gegenwärtig (1993) ca. 2,4 Mill. t KS- Trockenmasse an, das sind innerhalb der EU 40 \%. In der EU gelangen ca. $34 \%$ in die Landwirtschaft, $37 \%$ auf Deponien, $11 \%$ werden verbrannt, ca. $5 \%$ werden kompostiert (Bannick et al., 2002). In Deutschland sind die Relationen ähnlich (Bergs, 1998, ATV 1996). In Niedersachsen gelangen $74 \%$ in den Landbau (Gunreben, 2000).

Sollte die KS- Menge ansteigen, die nicht mehr in die Landwirtschaft gelangen soll, so hat das mehrere ökonomische Folgen. Eine Verbrennung wird sehr teuer und der Preis für das Abwasser, den die Bevölkerung bezahlen muss, steigt. Landwirte, die den KS als Nährstoffquelle genutzt haben, müssten Dünger kaufen. Diese Problematik ist besonders für das Nährelement Phosphor bedeutsam.

Wenn die bisherige KS- Menge von ca. 2,4 Mill. t einen P- Gehalt von ca. 15 kg P / t enthält (Schaaf und Janssen, 2000), so wären dies 36000000 kg / Jahr. Wenn die mittlere P- Abfuhr ca. 25 kg P / ha Ackerfläche und Jahr beträgt, so könnte die P- Versorgung von 1,4 Millionen ha Ackerland allein über den KS erfolgen. Das ist eine ökonomisch relevante Größe. Diese Kalkulation gilt sicher nicht nur für Deutschland, sondern generell. 
Außerdem sind die P- Vorräte der Welt begrenzt (Wachtel, 1976) und schon aus Gründen des Ressourcenschutzes sollte soviel KS wie möglich als Nährstoffquelle genutzt werden.

Die Landwirte müssen davon ausgehen, dass die KS die festgelegten Grenzwerte für Schadstoffe einhalten. Für deren Kontrolle sind sie nicht zuständig. Aber sie haben eigene Forderungen an die Qualität der KS. Sie sind vorrangig an den Nährstoffen in den KS interessiert. Hier in dieser Arbeit geht es um das Phosphat. Durch die Einführung der P- Elimination aus dem Klärwasser und seine Überführung in den $\mathrm{KS}$ in den Klärwerken mit Chemikalien wie Eisen- und Aluminium- Salzen bzw. Kalk sind die P- Einträge in die Fließgewässer stark zurückgegangen. Der Anteil der Kommunen an den insgesamt in die Fließgewässer eingeleiteten Phosphatmengen beträgt nur noch 30 \% (Umweltbundesamt, 1997). Dieser Effekt war und ist erwünscht. Das eliminierte Phosphat ist nun in den KS gebunden. Die Landwirte sind sehr an diesem Phosphat interessiert. Es sollte eine hohe Löslichkeit besitzen, damit es von den Pflanzen gut aufgenommen werden kann. Hierbei gibt es jedoch ein Problem, das von einzelnen Autoren unterschiedlich gesehen wird. Interessant ist vor allem die Beurteilung der Phosphordüngewirkung von KS aus Klärwerken mit P- Elimination durch Eisen-Salze.

Bemerkenswert sind die Untersuchungen von Baran (1985). Er setzte Klärschlammsuspensionen aus mechanisch / biologischer Reinigung zur Phosphateliminierung bis auf $1-2 \mathrm{mg} \mathrm{P} \mathrm{/} \mathrm{I} \mathrm{im} \mathrm{Filtrat} \mathrm{folgende} \mathrm{Chemikalien} \mathrm{zu:}$

1. nur $\mathrm{FeCl}_{3}$ oder

2. $\mathrm{FeCl}_{3}$ und dann zum abgetrennten Schlamm $\mathrm{CaO}$.

Die separierten Klärschlämme wurden getrocknet, fein gemahlen und dann verschiedenen Böden ( $\mathrm{P}$ - Gehaltsklasse A und B sowie pH-Werten 5,4 bis 7,7 ) zugesetzt und mit Weidelgras besät. Während das Weidelgras (7 Schnitte) in der 2. Variante weitgehend unabhängig vom Boden die gleichen P- Mengen aufnahm (92 - $103 \%$ ) wie aus Superphosphat (100\%), wurden auf den 3 Böden mit KS, die nur $\mathrm{FeCl}_{3}$ zur P- Fällung erhalten hatten, nur $51 \%$ (Karbonathaltiger Boden) bzw. weniger als 11 \% (Sandboden, Parabraunerde 
mit $\mathrm{pH}$-Werten < 5,5) des Phosphates im Vergleich zu Superphosphat aufgenommen. Die alleinige $\mathrm{FeCl}_{3}-$ Applikation zu dem biologisch aufbereiteten KS reduzierte also dessen P- Verfügbarkeit für die Graspflanzen in allen drei Böden im Vergleich zur kombinierten Anwendung von $\mathrm{FeCl}_{3}$ und $\mathrm{Ca}(\mathrm{OH})_{2}$. Diesem Verhalten der Pflanzen widersprechen aber die chemischen Kenngrößen der KS (nach Tabelle 3.4 und 3.5 von Baran):

\begin{tabular}{|c|c|c|c|}
\hline Variante & $\begin{array}{c}\text { Desorbierte } \\
\text { P- Menge } \\
(\mu \mathrm{g} \mathrm{P} / \mathrm{I}) \\
\text { (nach } 6 \text { Extraktionen) }\end{array}$ & $\begin{array}{c}\mathrm{H}_{2} \mathrm{O} \text { lösliches- } \\
\mathrm{P} \\
\text { (\% vom ges. } \mathrm{P})\end{array}$ & $\begin{array}{c}\text { austauschbares } \\
{ }^{32} \mathrm{P} \\
\text { (\% vom ges. } \mathrm{P} \text { ) }\end{array}$ \\
\hline 1. $\mathrm{FeCl}_{3}$ & 29 & 0,13 & 3,0 \\
\hline $\begin{array}{l}\text { 2. } \mathrm{FeCl}_{3}+ \\
\mathrm{Ca}(\mathrm{OH})_{2}\end{array}$ & 19 & 0,02 & 2,1 \\
\hline
\end{tabular}

Das Phosphat in dem KS nur mit $\mathrm{FeCl}_{3}-$ Fällung (1. Variante) war nach diesen Angaben also sogar chemisch besser löslich als in der $\mathrm{FeCl}_{3} / \mathrm{Ca}(\mathrm{OH})_{2}-$ Variante, obwohl die Pflanzen aus ihr weniger P aufgenommen hatten. Warum das Phosphat aus den Varianten nur mit $\mathrm{FeCl}_{3}$ - Zusatz von den Pflanzen weniger gut aufgenommen wurde als bei einem Zusatz von $\mathrm{FeCl}_{3}+\mathrm{Ca}(\mathrm{OH})_{2}$, bleibt hier somit unklar.

Die vergleichende Untersuchung von Suntheim und Dittrich (1996) zur PAufnahme von Mais aus einem sandigen Lehmboden mit einem P- Gehalt von 2,7 mg DL- P / $100 \mathrm{~g}$ und der Applikation verschiedener Klärschlämme ergab eine deutliche Unterlegenheit der P- Aufnahme der Pflanzen nach Applikation von Fe- haltigen KS im Vergleich zu Al- haltigem KS oder zu Bioschlamm bzw. Superphosphat.

In vergleichenden Untersuchungen mit Klärschlämmen, bei denen $\mathrm{Al}$ - und $\mathrm{Fe}$ Salze zur P- Fällung eingesetzt waren, ergab sich, dass Hafer aus KS mit FeFällung deutlich weniger $\mathrm{P}$ aufnahm als aus $\mathrm{KS}$ mit Al- Fällung bzw. Dicalciumphosphat (Werner, 1976). So lag die P-Ausnutzung aus dem 
eisenhaltigen KS Eutin auf dem Boden Gleichungen mit pH 7 um $18 \%$ niedriger als nach Dicalciumphosphatdüngung!

Aus den Untersuchungen von Frossard et al. (1996) mit zwei Böden (Tonboden, Lehmboden) und vier KS unterschiedlicher Fe: P- Verhältnisse mit Lolium perenne als Versuchspflanze geht folgendes hervor: Im Vergleich zur PDüngung mit $\mathrm{Ca}\left(\mathrm{H}_{2} \mathrm{PO}_{4}\right)_{2}$ nahmen die Graspflanzen auf dem Tonboden nur 62 bis $77 \%$ der P-Menge auf, wenn sie mit vier eisenhaltigen KS gedüngt waren. Auf dem Lehmboden lag der relative Anteil der P-Aufnahme bei nur $72-86 \%$ nach KS- Düngung im Vergleich zu $\mathrm{Ca}\left(\mathrm{H}_{2} \mathrm{PO}_{4}\right)_{2}$.

Aus all diesen Beispielen leitet sich ab, dass die zur Prüfung verwendeten Kulturpflanzen Mais, Weidelgras und Hafer offenbar nicht in der Lage waren, die gleichen P- Mengen aus KS mit P- Eliminierung im Abwasser durch Eisensalze aufzunehmen wie aus solchen mit Al- oder Ca- Konzentrierung.

Man kann davon ausgehen, dass in Deutschland 1. Mill. t Chemikalien in der Abwasserbehandlung, davon ca. $500000 \mathrm{t}$ Eisensalze, eingesetzt werden (Hahn, 2000, Scheffer, 2000). In den KS sind also Eisenverbindungen, die vermutlich in sehr unterschiedlichem Umfang Phosphat gebunden haben, enthalten. Daraus ergibt sich die Frage, welche P- Düngewirkung solche KS haben, wenn sie zur Düngung eingesetzt werden. Dabei interessiert nicht nur die P- Menge, die die Pflanzen aufnehmen, sondern auch die P- Verfügbarkeit im Boden. Denn, wenn eisenreiche KS in den Boden gelangen, ist denkbar, dass diese auch Phosphationen des Bodens binden, also dessen PVerfügbarkeit sogar senken können. Es war deshalb das Ziel der geplanten Untersuchungen, KS mit unterschiedlichen Eisengehalten als $P$ - Dünger einzusetzen und deren P- Düngewirksamkeit zu prüfen. Dabei sollten keine KS durch gezielte Fe- Salzzugaben selbst produziert werden, sondern KS geprüft werden, wie sie ganz konkret in Klärwerken mit variierter P- Elimination anfallen. 
Im Teil I der Untersuchungen sollen folgende Fragen geklärt werden:

- Wie verlaufen Trockenmassebildung und P- Entzug von Mais, Rotklee und Weidelgras, wenn sie auf Böden (Schluffböden, Sandböden, Quarzsand) mit hohen und niedrigem $\mathrm{P}$ - Gehalt nacheinander angebaut werden und $\mathrm{P}$ als $\mathrm{CaHPO}_{4}$ oder in Form von KS mit engem bzw. weitem molaren Fe: PVerhältnis einen Monat vor der Saat zum Mais angewendet werden?

- Wie verlaufen Wachstum und P- Entzug von Weidelgras, wenn dieses auf verschiedenen Böden (siehe oben) wächst und die P- Quellen 13 Monate vor der Saat ausgebracht werden?

- Wie verändern sich Parameter der P- Verfügbarkeit in den Böden (PSorption, Orthophosphatkonzentration der Bodenlösung) 1, 6 bzw. 12 Monate nach der P- Applikation?

Im Teil II soll die Frage geklärt werden, ob sich mit einem Spektrum von 13 Klärschlämmen unterschiedlichen molaren Fe: P- Verhältnisses (1:0,24 bis 1:7,94 entsprechend $4.2: 1$ bis $0,13: 1$ ) ein für Sandböden tolerables Fe: PVerhältnis der KS ermitteln lässt. Als Versuchspflanze dient Weidelgras.

Im Teil III soll die P- Düngewirkung in zwei ägyptischen Böden an Mais in einem Kurzzeitversuch getestet werden. 


\section{Teil I}

\section{Mehrjährige Experimente zur P-Aufnahme von Kulturpflanzen und zur P- Verfügbarkeit in Böden nach Applikation von zwei Klärschlämmen bzw. $\mathrm{CaHPO}_{4}$}

\subsection{Generelle Ziele}

(A) Klärschlämme mit unterschiedlichem molaren Fe: P- Verhältnis und Orthophosphat sind in verschiedene Böden einzubringen und kurzfristig (1 Monat) und längerfristig (12 Monate) im Boden zu inkubieren. Danach ist die PAufnahme verschiedener Kulturpflanzen ein- bzw. mehrjährig vergleichend zu prüfen. (Kapitel 2.2 und 2.3)

(B) Parallel dazu sollen Parameter der P - Verfügbarkeit in den Böden in längeren Zeiträumen (1, 6 bzw. 12 Monate) gemessen werden. Diese Parameter sollen zur Erklärung möglicherweise auftretender Unterschiede in der P - Aufnahme der Pflanzen dienen (Kapitel 2.4)

\subsection{Dreijähriges Experiment zur P - Aufnahme der Pflanzen}

\subsubsection{Vorbemerkungen und Versuchsziele}

Das Ziel dieses Experimentes war, Wachstum (Trockenmassebildung) und PAufnahme von den drei aufeinander folgenden Kulturpflanzen Mais, Rotklee und Weidelgras in Abhängigkeit von der P- Düngung mit eisenhaltigen Klärschlämmen (KS) im Vergleich zum "P- Mineraldünger" $\mathrm{CaHPO}_{4}$ in den Jahren 1999, 2000 und 2001 zu prüfen. Dabei wurde so vorgegangen, dass die P- Dünger in die Böden eingebracht und 4 Wochen später die erste Kultur, der Mais, ausgesät wurde. Die Auswahl der Kulturpflanzen hat folgende Begründung. Mais ist eine Pflanze, die auf eine P- Mangelsituation stärker reagiert als z.B. andere Kulturpflanzen (Römer et al. 1998). Sie müsste als Testpflanze auf die Nutzbarkeit von KS - P gut geeignet sein. Rotklee scheidet nach Gerke (1995) organische Säuren aus, wenn die P- Versorgung niedrig ist. Diese Pflanze könnte eventuell $P$, das an Fe- Oxide gebunden ist, mobilisieren. Deshalb kam sie als Testpflanze in Frage. Weidelgras hat den Vorteil, dass 
man mehrere Schnitte ernten und eventuell die P- Nachlieferung innerhalb eines Jahres verfolgen kann. Da ein P- Dünger auf Böden mit niedrigen PGehalten sicher am besten auf seine P- Düngewirkung geprüft werden kann, sind Böden ausgesucht worden mit möglichst niedrigem Gehalt an lactatlöslichem Phosphat. Aber der VDLUFA war auch daran interessiert, mehr über die KS- Wirkung auf Böden mit ausreichend hohen P- Gehalten zu erfahren (Merkel,1999 pers. Mitt.). Deshalb wurde auch ein Boden mit hohem P- Gehalt (Börry P-80) mit untersucht.

\subsubsection{Versuchsanlage und Durchführung}

\subsubsection{Benutzte Klärschlämme}

Die zwei benutzten Klärschlämme stammten aus den Kläranlagen von Göttingen (KS Gö) und Salzhausen (KS Sh). Alle Klärschlammproben wurden aus frischem Material im März 1999 gewonnen. Für die Analysen und Gefäßversuche wurden Unterproben gefriergetrocknet. Wichtige Kenndaten der zwei benutzten Klärschlämme sind in Tabelle 1 wiedergegeben. Im Klärwerk Salzhausen wurde nur $\mathrm{FeCl}_{3}$ zur P- Fällung im Belebungsbecken eingesetzt, während im Klärwerk Göttingen $\mathrm{FeSO}_{4}$ zur Anwendung kam. Außerdem wurde dem Schlamm in Göttingen bei der Konditionierung $\mathrm{FeCl}_{3}$ und $\mathrm{Ca}(\mathrm{OH})_{2}$ zugesetzt. Der Klärschlamm Göttingen (KS Gö) wurde vor der Applikation in die Gefäße gefriergetrocknet und gemahlen, um eine Homogenisierung des grobstrukturierten Klärschlammes zu erreichen und um gleiche KS- Mengen mit identischen P- Gehalten für die jeweiligen Wiederholungen einwiegen zu können. Diese Vorbehandlung war für den Klärschlamm Salzhausen nicht erforderlich. Dessen KS- Suspension (3,6\% TM) war homogen und konnte direkt volumetrisch abgemessen im Originalzustand in die Böden eingearbeitet werden. Die Trocknung von KS soll bis zu Trocknungstemperaturen von $400{ }^{\circ} \mathrm{C}$ keine Auswirkung auf die P- Löslichkeit und P- Wirksamkeit haben (Werner, 1976). Deshalb wurde nicht erwartet, dass die Gefriertrocknung negative oder positive Auswirkungen auf die P-Verfügbarkeit hat. Im Klärschlamm Göttingen betrug das molare Fe: $P$ Verhältnis 1:0,3 und im Klärschlamm Salzhausen 1:1,1. Es wurde das molare Fe: $\mathrm{P}$ Verhältnis berechnet, da $1 \mathrm{Mol} \mathrm{Fe}(\mathrm{OH})_{3}$ theoretisch $3 \mathrm{Mol}$ Phosphat binden kann. Das Verhältnis 1:0,3 wird als ein 
weites, das von 1: 1,1 als relativ enges Verhältnis angesehen. Infolge der Zufuhr von $\mathrm{Ca}(\mathrm{OH})_{2}$ bei der Konditionierung des Klärschlammes Göttingen werden bei dieser Anwendung mit $30 \mathrm{mg} \mathrm{P}$ gleichzeitig $493 \mathrm{mg} \mathrm{Ca} \mathrm{kg}^{-1}$ Boden appliziert. Bei dem Klärschlamm Salzhausen sind es nur $25 \mathrm{mg}$ Ca. Die $493 \mathrm{mg}$ Ca sind wie Tabelle 1 zeigt im wesentlichen basisch wirksam $(\mathrm{pH}=11,5)$, da $\mathrm{Ca}(\mathrm{OH})_{2}$ zur Konditionierung benutzt wird. Diese hohe Basizität dürfte Auswirkungen auf die pH-Werte der Böden und eventuell die P- Verfügbarkeit haben, was später (Diskussion der Ergebnisse) zu bedenken ist. Als mineralische Vergleichssubstanz wurde bewusst $\mathrm{CaHPO}_{4}$ und nicht das wasserlösliche Triplephosphat benutzt, da die Schlämme kaum wasserlösliches Phosphat enthalten dürften.

Tabelle 1: Kenndaten der gefriergetrockneten applizierten Klärschlämme (weitere Analysendaten vgl. Tabelle 8.3, Anhang)

\begin{tabular}{llcc}
\hline & & Göttingen (Gö) & Salzhausen $(\mathrm{Sh})$ \\
\hline $\mathrm{TM}$ & $(\%)$ & 32,4 & 3,6 \\
$\mathrm{P}$ & $\left(\mathrm{kg} \mathrm{t}^{-1} \mathrm{TM}\right)$ & 12,0 & 25,0 \\
$\mathrm{Fe}$ & $\left(\mathrm{kg} \mathrm{t}^{-1} \mathrm{TM}\right)$ & 65,0 & 39,0 \\
$\mathrm{Ca}$ & $\left(\mathrm{kg} \mathrm{t}^{-1} \mathrm{TM}\right)$ & 197,0 & 21,0 \\
$\mathrm{pH}$ & $\left(\mathrm{H}_{2} \mathrm{O}\right)$ & 11,5 & 7,0 \\
$\mathrm{Fe}: \mathrm{P}$ & Molar & $1: 0,3$ & $1: 1,1$ \\
\hline
\end{tabular}

\subsubsection{Benutzte Böden}

In den Versuchen sollte die Phosphataufnahme von Pflanzen aus verschiedenen Böden, die mit Klärschlamm gedüngt waren, untersucht werden. Deshalb sollten die Versuchsböden möglichst unterschiedliche Bodenarten mit differenzierten Phosphatgehalten repräsentieren. So wurden drei bindige Böden (tonige Schluffe aus Börry P-0, P-80 und Elliehausen), zwei Sandböden (Rehburg, Düshorn) und Quarzsand als P- freies Substrat ausgewählt. Die Kenndaten der eingesetzten Böden gibt Tabelle 2 wieder. Die benutzten Böden entstammen landwirtschaftlich genutzten Ackerflächen. Das Bodenmaterial wurde meistens aus der Ackerkrume $(0-30 \mathrm{~cm})$ des jeweiligen Standortes 
entnommen. Die beiden Böden Börry P-0 und P-80 stammen aus einem längerfristigen statischen P- Düngungsversuch aus dem Gebiet um Hameln (Parabraunerde aus Löß) und enthalten mäßige $\left(46 \mathrm{mg} \mathrm{kg}^{-1}\right)$ bis hohe PGehalte $\left(110 \mathrm{mg} \mathrm{kg}^{-1}\right)$ nach der CAL- Methode. Der Boden Elliehausen ist ein sehr P- armes und humusarmes Material aus dem C - Horizont einer Parabraunerde aus Löß (westlich von Göttingen). Die Böden aus Rehburg (Kreis Nienburg) und Düshorn (Kreis Fallingbostel) sind anlehmige Sand- bzw. Sandböden pleistozänen Ursprungs mit relativ niedrigen P- Gehalten. Außerdem kam Quarzsand (Sandgrube Weferlingen, Kreis Haldensleben) als Substrat zur Anwendung. Die pH-Werte $\left(\mathrm{CaCl}_{2}\right)$ der drei schweren Böden liegen im neutralen $(6,3$ bis 7,2$)$ bzw. im basischen Bereich $(7,7)$. Die zwei Sandböden zeigen saure Reaktion ( $\mathrm{pH} 4,3$ bis 4,9$)$.

Tabelle 2: Kenngrößen der Versuchsböden

\begin{tabular}{lccccccc}
\hline Ort & $\begin{array}{c}\text { Ton } \\
\%\end{array}$ & $\begin{array}{c}\text { Schluff } \\
\%\end{array}$ & $\begin{array}{c}\text { Sand } \\
\%\end{array}$ & $\begin{array}{c}\mathrm{CaCO}_{3} \\
\%\end{array}$ & $\begin{array}{c}\mathrm{pH} \\
\mathrm{CaCl}_{2}\end{array}$ & $\begin{array}{c}\text { Humus } \\
\%\end{array}$ & $\begin{array}{c}\mathrm{CAL}^{1} / \mathrm{DL}^{2} \\
\mathrm{mg} \mathrm{P} \mathrm{kg}^{-1}\end{array}$ \\
\hline Börry P-0 & 20 & 75 & 5 & 1,3 & 7,2 & 2,22 & 46 \\
Börry P-80 & 20 & 75 & 5 & 0,9 & 6,3 & 2,22 & 110 \\
Elliehausen & 16 & 75 & 9 & 13,3 & 7,7 & 0,50 & 6 \\
Rehb.-Loccum & 8 & 13 & 79 & n.b. & 4,3 & 7,20 & 17 \\
$\begin{array}{l}\text { Düshorn } \\
\text { (Brache) }\end{array}$ & 3 & 13 & 84 & n.b. & 4,9 & 4,00 & 34 \\
\hline
\end{tabular}

1) bei Lehmböden, ${ }^{2)}$ bei Sandböden, n.b. = nicht bestimmt, ${ }^{3)}$ Jährliche P-Düngung der Parzellen in $\mathrm{kg} \mathrm{ha}^{-1}$

\subsubsection{Anlage des Gefäßversuchs zur P-Aufnahme}

\section{Versuchsjahr 1999 (Mais)}

Die fünf verschiedenen Böden und Quarzsand wurden mit dem fein gemahlenen und gefriergetrockneten Klärschlamm Göttingen bzw. mit der Suspension Klärschlamm Salzhausen in drei P- Stufen gedüngt. Als Bezugsbasis galten 5t TM des Klärschlamms Göttingen ha-1, weil in 
Deutschland nach der Klärschlammverordnung alle 3 Jahre 5 t TM ausgebracht werden dürfen. Nach Tab. 1 enthalten $5 \mathrm{t} \mathrm{KS} \mathrm{Gö} 60 \mathrm{~kg} \mathrm{P}$. Auf die Oberfläche eines Mitscherlichgefäßes $\left(314 \mathrm{~cm}^{2}\right)$ umgerechnet wären dies $187 \mathrm{mg} \mathrm{P} \mathrm{Geäß}^{-1}$ d.h. ca. $30 \mathrm{mg} \mathrm{P} \mathrm{kg}{ }^{-1}$ Boden. Vom KS Salzhausen wurde Schlamm mit der gleichen $\mathrm{P}$ - Menge eingesetzt.

Da auch sehr P- arme Substrate wie Quarzsand und der Boden Elliehausen genutzt werden sollten, war zu erwarten, dass auch schon geringere P- Gaben positive Effekte (Biomassebildung) bewirken könnten. Deshalb wurde auch mit kleineren P- Gaben: 44 bzw. 87 mg P Gefäß ${ }^{-1}$ gearbeitet. So ergab sich für die $P$ - Düngung folgendes Schema (Tabelle. 3)

Tabelle 3: P- Düngungsvarianten

\begin{tabular}{lcccc}
\hline Boden & \multicolumn{5}{c}{$\mathrm{P}-$-Gaben $\left(\mathrm{mg} P\right.$ Gefäß ${ }^{-1} / \mathrm{mg} \mathrm{P} \mathrm{kg}^{-1}$ Boden) } \\
& $0 / 0$ & $44 / 7$ & $87 / 15$ & $187 / 30$ \\
\hline Börry P-0 & $*$ & & & $*$ \\
Börry P-80 & $*$ & & & $*$ \\
Elliehausen & $*$ & $*$ & $*$ & $*$ \\
Rehburg- Loccum & $*$ & $*$ & $*$ & $*$ \\
Düshorn Brache & $*$ & & & $*$ \\
Quarzsand & & $*$ & $*$ & $*$ \\
\hline
\end{tabular}

Auf Basis der entsprechenden Schlammmengen und deren Fe- Gehalten ergaben sich für die Gefäße mit $187 \mathrm{mg} P$ folgende Fe- Mengen: im KS Gö: 157, im KS Sh: $44 \mathrm{mg} \mathrm{kg}^{-1}$ Boden. Vor dem Befüllen der Mitscherlichgefäße wurden in die jeweils $6 \mathrm{~kg}$ Boden folgende Nährstoffmengen eingearbeitet:

? 44, 87 bzw. 187 mg P (= 100, 200 bzw. 428 mg P $\left.{ }_{2} \mathrm{O}_{5}\right)$ als $\mathrm{CaHPO}_{4}$, oder KS Gö bzw. KS Sh,

? $0,6 \mathrm{~g} \mathrm{~N}$ als Calciumnitrat $\mathrm{Ca}\left(\mathrm{NO}_{3}\right)_{2} \cdot 4 \mathrm{H}_{2} \mathrm{O}$,

? $1,0 \mathrm{~g} \mathrm{~K}$ als Kaliumsulfat $\mathrm{K}_{2} \mathrm{SO}_{4}$, 
$0,2 \mathrm{~g} \mathrm{Mg}$ als Magnesiumsulfat $\mathrm{MgSO}_{4} \cdot 7 \mathrm{H}_{2} \mathrm{O}$,

Mikronährstoffe nach Hoagland 1:10 verdünnt, $10 \mathrm{ml}^{\text {Gefäß }}{ }^{-1}$ (Lösung $A+B$ ) (vgl. Schilling, 2000)

Fe als $\mathrm{Fe}$ - Sequestren, 6\%- ige Lösung, 1:20 Verdünnung, $10 \mathrm{ml} \mathrm{Gefäß}^{-1}$.

Während des Versuchsablaufs wurden alle Gefäße dreimal mit 0,3 g N als Calciumnitrat und die Gefäße mit den Böden Börry insgesamt viermal mit 0,3 g $N$ Gefäß ${ }^{-1}$ nachgedüngt. Die Varianten wurden in vierfacher Wiederholung angesetzt. 4 Wochen nach Versuchsansatz wurden die Gefäße mit Maissamen einer frühreifen Hybride (noch ohne Sortenname) pro Gefäß besät. Es wuchsen 4 Pflanzen je Gefäß. Die Pflanzen wuchsen 90 Tage. Gegossen wurde auf ca. $70 \%$ der maximalen Wasserkapazität. Die Wasserversorgung erfolgte täglich mit entmineralisiertem Wasser. Die Gefäße standen im Drahthaus des Instituts für Agrikulturchemie unter Freiluftbedingungen. Zu Versuchsende wurden die Pflanzen in Körner und Restpflanze getrennt, bei $105^{\circ} \mathrm{C}$ getrocknet, gemahlen und der P- Analyse zugeführt. Anschließend blieben die Gefäße in der Drahthalle stehen und der Witterung ausgesetzt, so dass mineralischer Stickstoff ausgewaschen werden konnte.

\section{Versuchsjahr 2000 (Rotklee)}

Die P- Nachwirkung der KS wurde im zweiten Versuchsjahr mit Rotklee untersucht. Am 13. März 2000 wurden die Gefäße entleert, grobe Wurzelreste des Maises entfernt und die folgenden Nährstoffmengen in die Böden eingemischt:

$0,6 \mathrm{~g} \mathrm{~N}$ als $\mathrm{NH}_{4} \mathrm{NO}_{3}$,

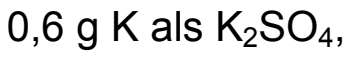

$0,1 \mathrm{~g} \mathrm{Mg}$ als $\mathrm{MgSO}_{4} \cdot 7 \mathrm{H}_{2} \mathrm{O}$. 
Am 24. April 2000 erfolgte die Aussaat des Rotklees Sorte TITUS ( $0,28 \mathrm{~g}$ pro Gefäß). Die N- Düngung erfolgte in allen Gefäßen, da unklar war, inwieweit eine Inokulation der Böden mit Rhizobien (Elliehausen, Quarzsand) erfolgreich sein würde oder nicht. Alle Pflanzen sollten gleiche Startbedingungen haben. Ausgesät wurde aber nur in die Gefäße ohne $P$ und mit $187 \mathrm{mg} \mathrm{P}$ Gefäß $^{-1}$, weil die niedrigen $\mathrm{P}$ - Gaben von 44 bzw. $88 \mathrm{mg} \mathrm{P} \mathrm{Gefäß}^{-1}$ im ersten Anbaujahr auf den Böden mit niedrigem P- Gehalt Pflanzen mit deutlichem P- Mangel hervorgebracht hatten. Die Pflanzen wurden wieder in der Vegetationshalle wie oben beschrieben kultiviert. Es wurden 3 Schnitte geerntet (am 13.07, am 23.08 und am 24.10.2000), die ebenfalls getrocknet, gewogen und gemahlen zur Analyse gelangten. Anschließend blieben die Gefäße in der Drahthalle stehen.

\section{Versuchsjahr 2001 (Weidelgras)}

Im Jahr 2001 erfolgte in den Gefäßen, die in der Drahthalle standen, ein Nachbau mit Weidelgras, um weiter die Nachwirkung der P-Düngung im dritten Anbaujahr zu prüfen. Die Gefäße wurden entleert. Die Wurzelreste verblieben im Boden. Am 11. April 2001 wurden folgende Nährstoffmengen in die Böden eingemischt:

$0,6 \mathrm{~g} \mathrm{~N}$ als $\mathrm{NH}_{4} \mathrm{NO}_{3}$

$0,8 \mathrm{~g} \mathrm{~K}$ als $\mathrm{K}_{2} \mathrm{SO}_{4}$,

$0,15 \mathrm{~g} \mathrm{Mg}$ als $\mathrm{MgSO}_{4} \cdot 7 \mathrm{H}_{2} \mathrm{O}$.

Dann erfolgte die Aussaat des Weidelgrases Sorte LIRASAND $(0,14 \mathrm{~g}$ pro Gefäß). Ausgesät wurde nur in die Gefäße ohne $P$ und mit $187 \mathrm{mg} \mathrm{P}$ Gefäß $^{-1}$. Die Pflanzen standen unter den gleichen äußeren Bedingungen wie der Mais und der Rotklee. Es wurden 3 Schnitte geerntet (14. Juni, 14. Juli, 9. August). Nach dem ersten und zweiten Schnitt sind 0,6 g N in Form von Ammoniumnitrat nachgedüngt worden. Nach Ermittlung der Trockenmasse kam das gemahlene Material zu P- Analyse. 


\subsubsection{Pflanzenanalyse auf Phosphor}

Zur Bestimmung der Phosphatgehalte wurden die getrockneten Pflanzenproben fein vermahlen und nass verascht. $200 \mathrm{mg}$ Pflanzenmaterial wurden in einem Erlenmeyerkolben mit $5 \mathrm{ml}$ eines Gemisches aus konzentrierter $\mathrm{HNO}_{3}: \mathrm{HCLO}_{4}$ : $\mathrm{H}_{2} \mathrm{SO}_{4}$ im Verhältnis 7:2:1 (v: v: v) versetzt und auf dem Sandbad mit abgestuften Temperaturerhöhungen von $80-220^{\circ} \mathrm{C}$ aufgeschlossen. Die Aufschlusslösung wurde in $25 \mathrm{ml}$ Messkolben aufgenommen und daraus $5 \mathrm{ml}$ Aliquot zur Analyse benutzt. Die P- Gehalte wurden spektralphotometrisch mit der Molybdat- Vanadat- Methode nach Scheffer und Pajenkamp (1952) ermittelt. Die Messung erfolgte bei einer Wellenlänge von $436 \mathrm{~nm}$.

\subsubsection{Ergebnisse}

Gefäßversuche mit Mais (1. Versuchsjahr): Sprossmassebildung und PEntzüge

\section{Maisanbau auf Boden mit hohem P-Gehalt}

Abbildung 1 zeigt, dass zwar durch die Zufuhr von $\mathrm{CaHPO}_{4}$ bzw. durch Klärschlamm der Ertrag auf dem hoch mit $P$ versorgten Boden Börry P-80 um 7 bis $12 \%$ gegenüber der ungedüngten Kontrolle anstieg, dass diese Anstiege aber statistisch nicht signifikant waren.

Die statistische Analyse der P-Entzüge zeigt ein interessantes Ergebnis, denn die P-Aufnahme aus der Variante KS Gö war identisch mit der der Kontrolle (0Variante) und gleichzeitig signifikant geringer als die der Ca-P-Variante. Das heißt, der Mais hatte offenbar aus dem KS Gö zumindest rechnerisch kein $\mathrm{P}$ aufgenommen. Dessen P-Verfügbarkeit für die Maispflanzen war also offenbar die gleiche oder eine geringere wie die des Bodenphosphates, und sie war deutlich geringer als die von $\mathrm{CaHPO}_{4}$. Die Verfügbarkeit des $\mathrm{P}$ aus KS Sh lag zwischen den beiden anderen P-Düngern. Die P-Verfügbarkeit der 3 P-Quellen zeigte also folgende Reihenfolge: $\mathrm{CaHPO}_{4}>\mathrm{KS} \mathrm{Sh}>\mathrm{KS}$ Gö. 

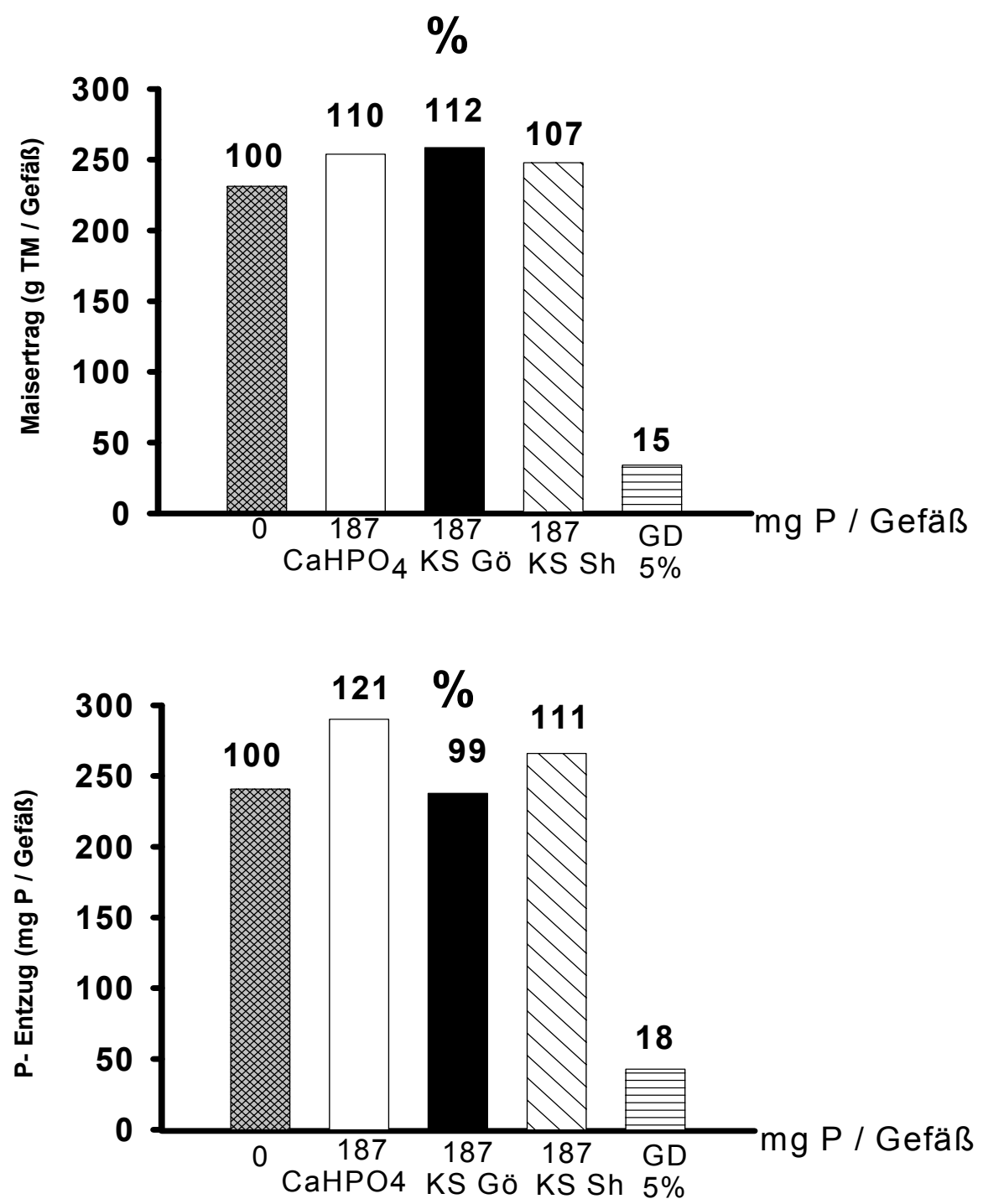

Abbildung 1: Abhängigkeit des Maisertrages und des $P$ - Entzuges von der $P$ - Applikation zum Boden Börry $P$ - 80

Maisanbau bei niedrigem und mäßigem P-Gehalt der Böden bzw. Substrate

Während, wie zu erwarten, auf einem Boden der Gehaltsklasse C/D durch P. Düngung trotz eventuell erhöhter P-Aufnahmen keine signifikanten Ertragswirkungen zu erwarten sind, sollten diese aber auf Böden bzw. Substraten mit niedriger bzw. mäßiger P-Versorgung auftreten. Das trifft für alle 
$\mathrm{CaHPO}_{4}$-Varianten tatsächlich zu, sowohl auf den sehr P-armen Substraten Quarzsand und dem Lößboden Elliehausen (Abbildung 2, 3) als auch auf den Böden Rehburg, Düshorn und Börry P-0 (Abbildung 4, 5, 6) im Vergleich zur ungedüngten Variante (bei Quarzsand die P-44 Variante). Dabei verhalten sich die Zuwächse der Erträge meist sehr ähnlich wie die P-Entzüge. Damit kann die P-Düngewirkung der Klärschlämme (TM-Bildung und P-Entzug) mit der der $\mathrm{CaHPO}_{4}$-Variante verglichen und beurteilt werden. Lässt man den Quarzsand zunächst aus der Betrachtung der übrigen Vergleichsglieder heraus - bei beiden Klärschlämmen war dort die relative P-Aufnahme (20-37\%) und die relative TM-Bildung (15-37\%) sehr niedrig - und betrachtet zunächst die Wirkung des KS Sh mit seinem engen Fe:P - Verhältnis von 1:1,2, so zeigt sich folgendes:
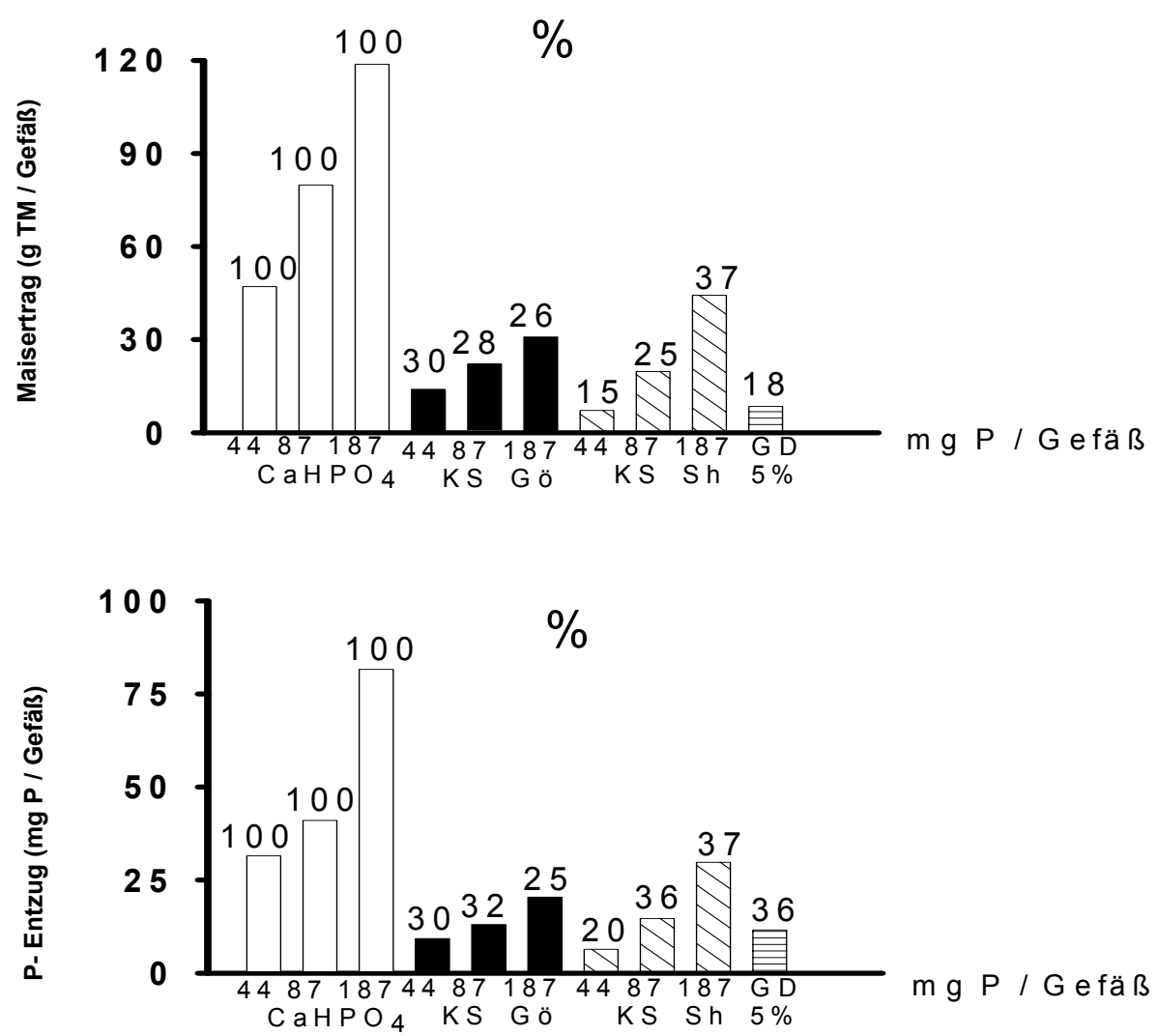

A bbildung 2: Abhängigkeit des $M$ aisertrages und des $P$ Entzuges von derP - Applikation zum Quarzsand 
Mit KS Sh wurden sowohl bei gestaffelter P-Zufuhr (Elliehausen und Rehburg, Abbildung 3,4$)$ als auch bei dem besser mit $P$ versorgten Boden Düshorn (Abbildung 5) stets TM-Erträge erzielt, die dem mit $\mathrm{CaHPO}_{4}$-Zufuhr erzielten sehr ähnlich waren. Nur auf dem Boden Börry P-0 (Abbildung 6) reichten die Erträge nicht an die der $\mathrm{CaHPO}_{4}$-Variante heran, obwohl sie auch hier signifikant über der der Kontrolle lagen. Die prinzipiell gleiche Aussage gilt auch für die P-Entzüge. Bei vergleichbaren Düngungsvarianten wurden auch vergleichbare P-Entzüge erreicht - auch im Boden Börry P-0.
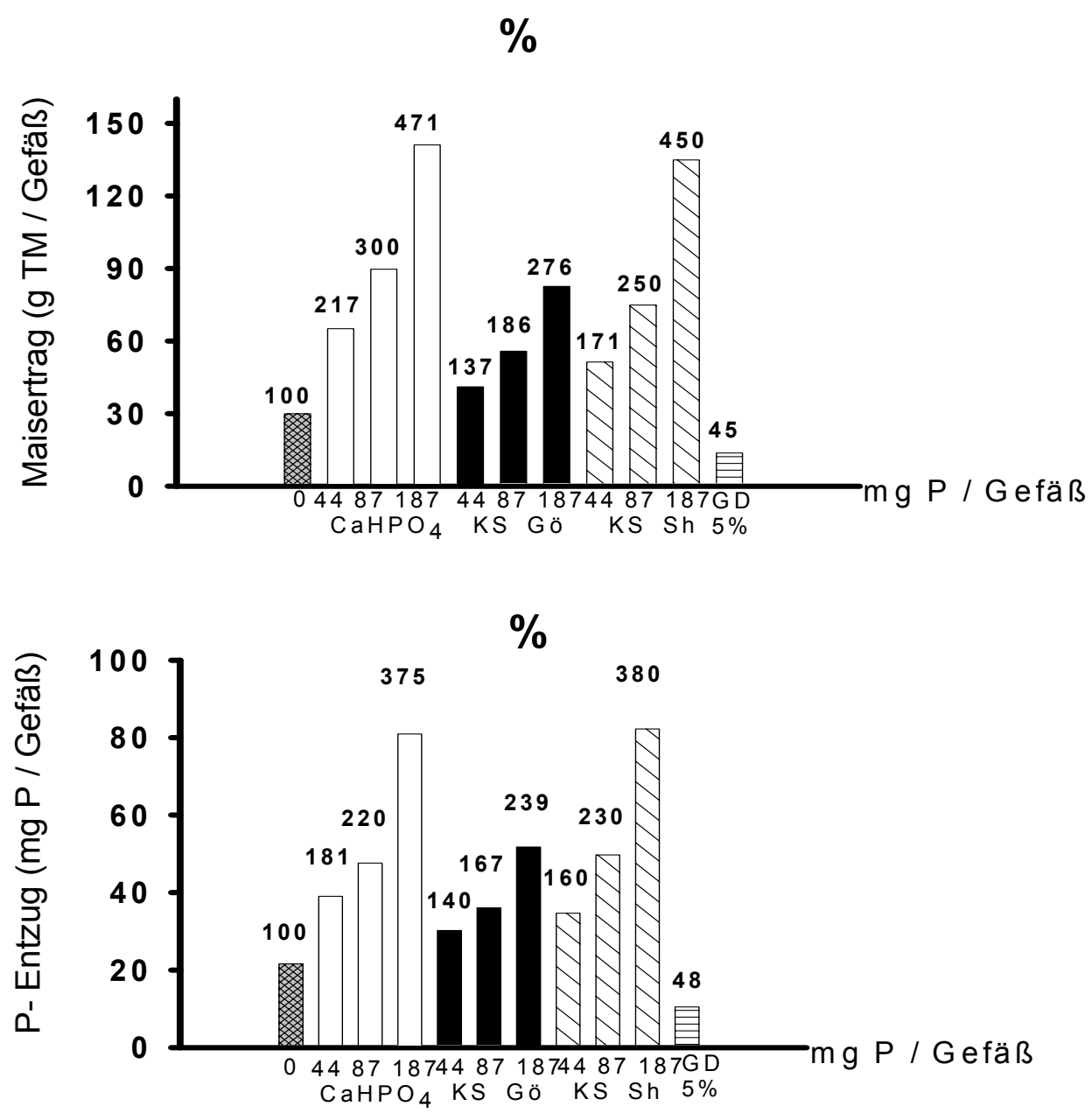

Abbildung 3: Abhängigkeit des Maisertrages und des $P$ - Entzuges von der P - Applikation zum Boden Elliehausen 

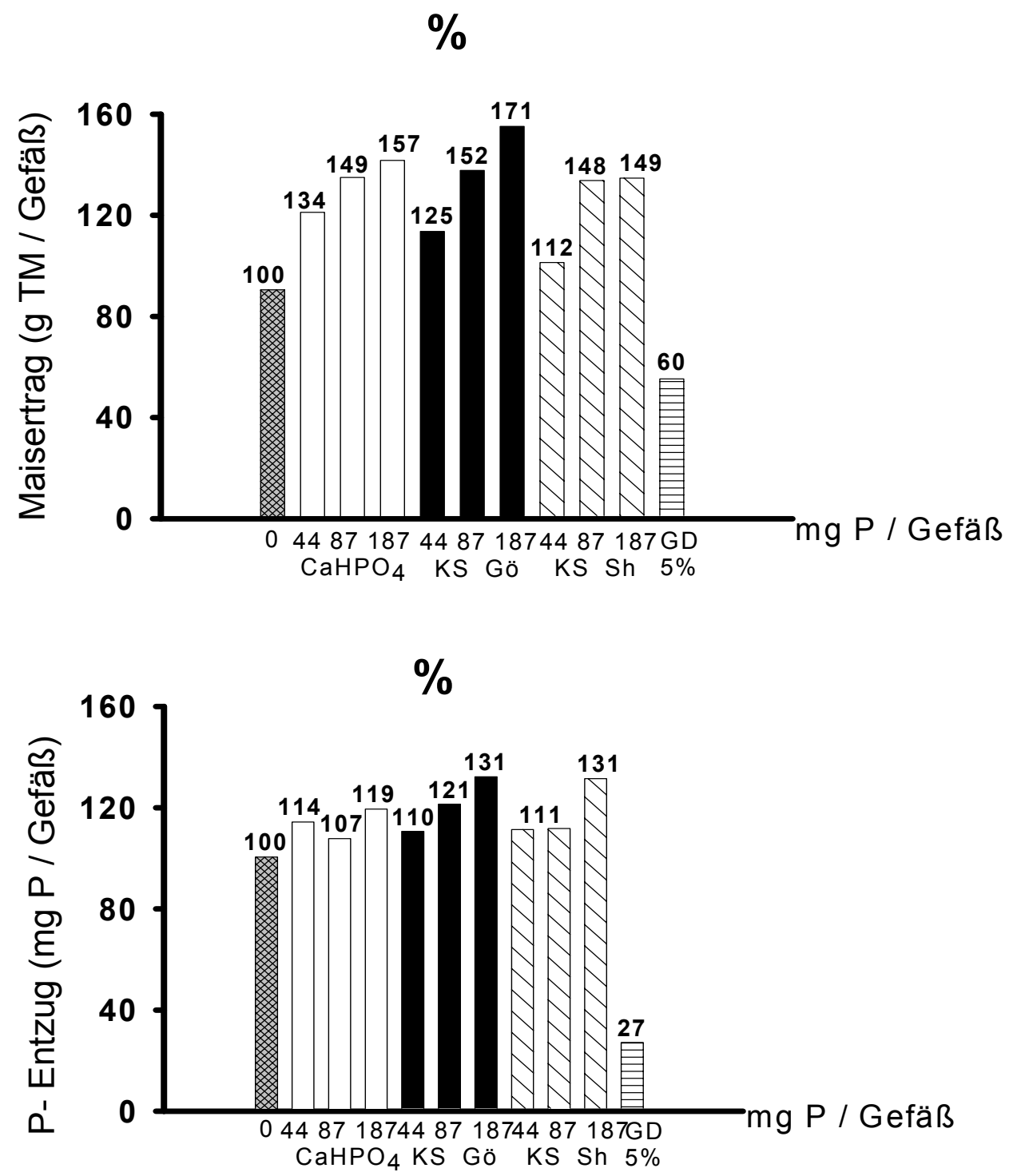

Abbildung 4: Abhängigkeit des Maisertrages und des $P$ - Entzuges von der P - Applikation zum Boden Rehburg-Loccum

Der Vergleich der P-Düngungsvarianten zwischen $\mathrm{CaHPO}_{4}$ und $\mathrm{KS}$ Gö fällt viel differenzierter aus. Bezüglich des TM- Ertrages bleibt die KS Gö - Variante nicht nur auf dem Quarzsand, sondern auch auf den Lößböden Elliehausen (Abbildung 3) und Börry P-0 (Abbildung 6) signifikant hinter der $\mathrm{CaHPO}_{4}$ Variante zurück. Sie übertrifft diese aber auf dem Boden Rehburg (P-187 mgVariante, Abbildung 4) tendenziell und auf dem Boden Düshorn (Abbildung 5) signifikant um $21 \%$. Auch die P-Entzüge zeigen ein differenziertes Bild. Während auf dem Quarzsand, den Lößböden Elliehausen und Börry P-0 
(Abbildung 2, 3, 6) die P-Aufnahme stets hinter der aus $\mathrm{CaHPO}_{4}$ zurück bleibt (wie die Erträge), ist sie auf den Böden Rehburg und Düshorn (Abbildung 4, 5) der $\mathrm{CaHPO}_{4}$-Variante sehr ähnlich. Diese Resultate sind später zu diskutieren.
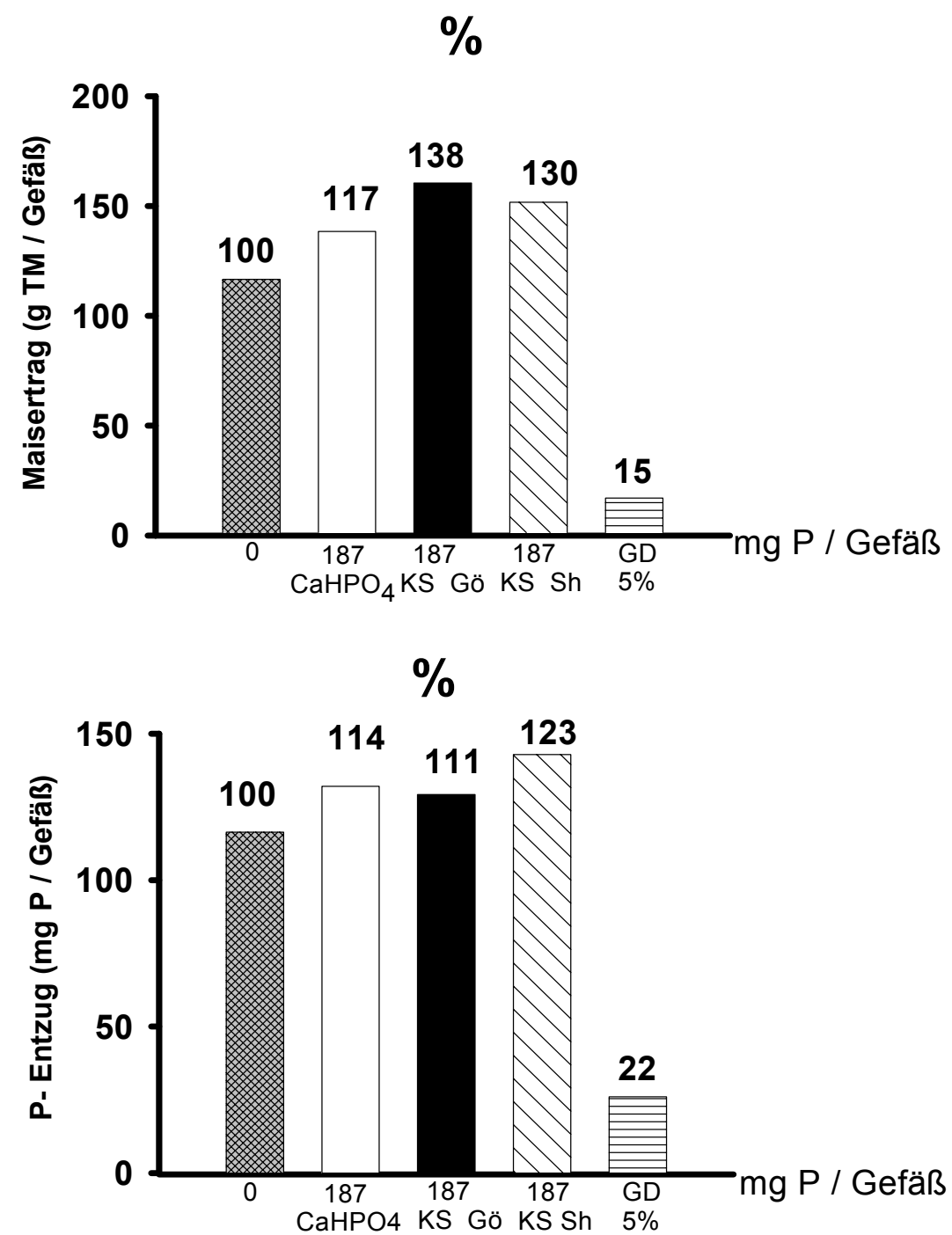

Abbildung 5: Abhängigkeit des Maisertrages und des $P$ - Entzuges von der P - Applikation zum Boden Düshorn (Brache) 

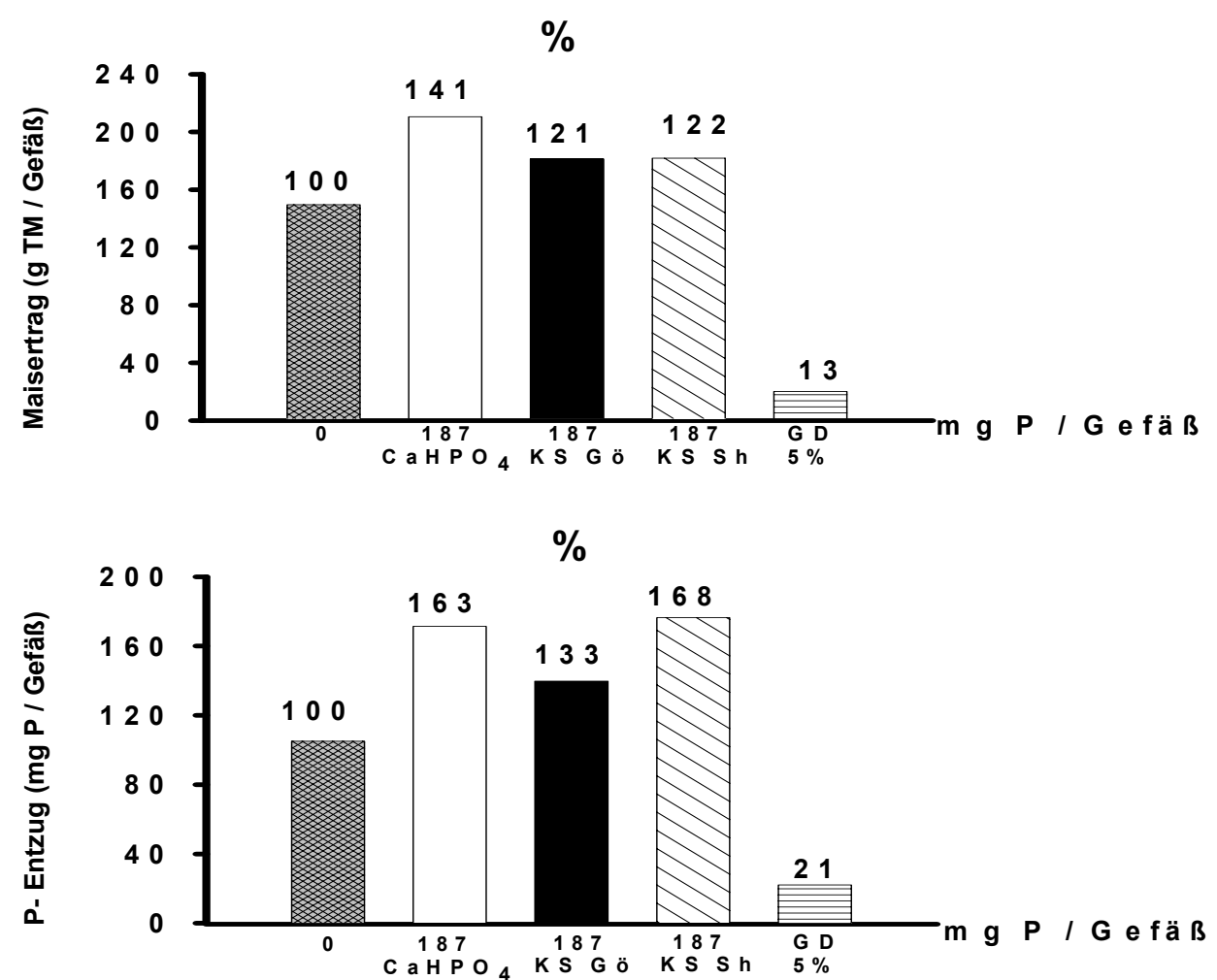

A b b ildung 6: A bhängigkeit des $M$ a is ertrages und des $P$ - Entzuges von derP-Applikation zum Boden Börry $P$ - O

Gefäßversuch mit Rotklee (2. Versuchsjahr): Sprossmassebildung und P. Entzüge

Tabelle 4 zeigt die Erträge von Rotklee im zweiten Versuchsjahr. Auf dem Boden in GK C/D (Börry P-80) trat wie beim Mais keine Ertragssteigerung ein. Die Erträge in der KS Sh- Variante und in der Ca-P-Variante waren genauso hoch wie die der Kontrolle (95 bzw. 96 \%). Dagegen fiel die KS Gö- Variante deutlich ab (87\%).

Prinzipiell ähnlich ist das Ergebnis mit den Böden in GK A und B. In allen Fällen lagen die Erträge der KS Gö- Variante niedriger als die der Ca-P- Variante und signifikant niedriger als die der KS Sh- Variante. Die Erträge sind ein Spiegelbild der vorliegenden P-Entzüge (Tabelle 5). 
Tabelle 4: Relativerträge der Rotkleetrockenmasse (Summe von 3 Schnitten) nach der P-Düngung in Höhe von $187 \mathrm{mg} P$ je Gefäß zum Mais (Vorfrucht)

\begin{tabular}{|c|c|c|c|c|c|c|c|c|}
\hline \multirow{2}{*}{$\begin{array}{l}\text { Bodenart/ } \\
\text { Substrat }\end{array}$} & \multirow[t]{2}{*}{ Standort } & \multirow[t]{2}{*}{$\mathrm{GK}^{1)}$} & \multicolumn{2}{|c|}{ Ohne P } & \multirow{2}{*}{$\begin{array}{l}\mathrm{CaHPO}_{4} \\
\text { relativ }\end{array}$} & \multirow{2}{*}{$\begin{array}{l}\text { KS Gö } \\
\text { relativ }\end{array}$} & \multirow{2}{*}{$\begin{array}{l}\text { KS Sh } \\
\text { relativ }\end{array}$} & \multirow{2}{*}{$\begin{array}{l}\mathrm{GD}^{2)} \\
5 \%\end{array}$} \\
\hline & & & $g$ & relativ & & & & \\
\hline Ton.Schluff & $\begin{array}{l}\text { Börry } \\
\text { P-80 }\end{array}$ & C/D & 78 & 100 & 96 & 87 & 95 & 7 \\
\hline Ton.Schluff & $\begin{array}{l}\text { Börry } \\
\text { P-0 }\end{array}$ & $\mathrm{B} / \mathrm{C}$ & 50 & 100 & 130 & 121 & 136 & 14 \\
\hline Ton.Schluff & $\begin{array}{l}\text { Ellie- } \\
\text { hausen }\end{array}$ & A & 20 & 100 & 126 & 123 & 167 & 32 \\
\hline Lehm.Sand & Rehburg & $A$ & 50 & 100 & 113 & 98 & 106 & 8 \\
\hline Sand & Düshorn & B & 54 & 100 & 109 & 106 & 113 & 5 \\
\hline Quarzsand & $\begin{array}{l}\text { Wefer } \\
\text { lingen }\end{array}$ & $A$ & --- & & $100^{3)}$ & 45 & 72 & 25 \\
\hline
\end{tabular}

1) Gehaltsklasse, ${ }^{2)}$ Tukey test, ${ }^{3)}$ absolut $=14 \mathrm{~g} /$ Gefäß

Tabelle 5: Relative P-Entzüge der Rotkleepflanzen (Summe von 3 Schnitten) als 2. Kultur nach einer P-Düngung von $187 \mathrm{mg} P / G e f a ̈ ß ~ z u$ Versuchsbeginn

\begin{tabular}{|c|c|c|c|c|c|c|c|}
\hline \multirow{2}{*}{$\begin{array}{l}\text { Boden/ } \\
\text { Substrat }\end{array}$} & \multirow[t]{2}{*}{ Standort } & \multicolumn{2}{|c|}{ Ohne P } & \multirow{2}{*}{$\begin{array}{c}\mathrm{CaHP} \\
\mathrm{O}_{4} \\
\text { relativ }\end{array}$} & \multirow{2}{*}{$\begin{array}{l}\text { KS Gö } \\
\text { relativ }\end{array}$} & \multirow{2}{*}{$\begin{array}{l}\text { KS Sh } \\
\text { relativ }\end{array}$} & \multirow{2}{*}{$\begin{array}{l}\mathrm{GD}^{1)} \\
5 \%\end{array}$} \\
\hline & & $\mathrm{mg}$ & relativ & & & & \\
\hline Ton.Schluff & Börry P-80 & 204 & 100 & 100 & 77 & 98 & 10 \\
\hline Ton.Schluff & Börry P-0 & 61 & 100 & 159 & 131 & 158 & 17 \\
\hline Ton.Schluff & Elliehausen & 15 & 100 & 167 & 181 & 213 & 21 \\
\hline Lehm.Sand & Rehburg & 63 & 100 & 126 & 104 & 133 & 13 \\
\hline Sand & Düshorn & 70 & 100 & 132 & 115 & 129 & 11 \\
\hline Quarzsand & Weferlingen & - & - & $100^{2)}$ & 49 & 90 & 34 \\
\hline \multicolumn{8}{|c|}{ Mittelwert der relativen P-Entzüge: } \\
\hline \multicolumn{3}{|c|}{5 Böden und Quarzsand } & - & 100 & 84 & 105 & \\
\hline \multicolumn{3}{|c|}{5 Böden ohne Quarzsand } & - & 100 & 89 & 107 & \\
\hline
\end{tabular}

1) Tukey test, ${ }^{2)}$ absolut $=8,5 \mathrm{mg} \mathrm{P} / \mathrm{Gefäß}$ 
Auf allen Böden, außer Elliehausen, hatte der Rotklee nach KS Gö- Düngung deutlich weniger $\mathrm{P}$ aufgenommen als aus $\mathrm{CaHPO}_{4}$ bzw. aus KS Sh. Der Boden Elliehausen machte eine Ausnahme. Hier gab es im Vergleich zu $\mathrm{CaHPO}_{4}$ weder signifikante Differenzen beim Ertrag noch bei den P-Entzügen.

\section{Gefäßversuch mit Weidelgras (3. Versuchsjahr): Sprossmassebildung und P- Entzüge}

In 3. Versuchsjahr wurde in den Gefäßen, die zu Beginn die höchste P- Gabe erhalten hatten (187 mg P Gefäß ${ }^{-1}$ d.h. ca. $30 \mathrm{mg} \mathrm{P} \mathrm{kg}^{-1}$ Boden) Weidelgras angebaut. Tabelle 6 zeigt die Gesamttrockenmasseerträge der 3 Schnitte und Tabelle 7 die Gesamt P- Entzüge.

Tabelle 6: Relativerträge der Weidelgrastrockenmasse (Summe von 3 Schnitten) nach der P-Düngung in Höhe von $187 \mathrm{mg} P$ je Gefäß zu Versuchsbeginn

\begin{tabular}{llccccccc}
\hline $\begin{array}{l}\text { Bodenart/ } \\
\text { Substrat }\end{array}$ & Standort & $\mathrm{GK}^{1)}$ & Ohne P & $\mathrm{CaHPO}_{4}$ & $\begin{array}{l}\mathrm{KS} \\
\mathrm{Gö}\end{array}$ & $\begin{array}{c}\mathrm{KS} \\
\text { Sh }\end{array}$ & $\mathrm{GD}^{2)}$ \\
& & & $\mathrm{g}$ & relativ & relativ & relativ & relativ & $5 \%$ \\
\hline Ton.Schluff & Börry P-80 & $\mathrm{C} / \mathrm{D}$ & 84 & 100 & 101 & 96 & 102 & 14 \\
Ton.Schluff & Börry P-0 & $\mathrm{B} / \mathrm{C}$ & 25 & 100 & 149 & 120 & 140 & 33 \\
Ton.Schluff & Elliehausen & $\mathrm{A}$ & 4 & 100 & 170 & 195 & 295 & 29 \\
Lehm.Sand & Rehburg & $\mathrm{A}$ & 22 & 100 & 149 & 188 & 154 & 19 \\
Sand & Düshorn & $\mathrm{B}$ & 36 & 100 & 143 & 133 & 138 & 9 \\
Quarzsand & Weferlingen & A & --- & --- & $100^{3)}$ & 128 & 202 & 42 \\
\hline
\end{tabular}

1) Gehaltsklasse, ${ }^{2)}$ Tukey test, ${ }^{3)}$ absolut $=14 \mathrm{~g} /$ Gefäß

Wie erwartet trat nach $\mathrm{P}$ - Düngung auf dem hoch mit $\mathrm{P}$ versorgten Boden Börry P-80 keine Ertragssteigerung ein. Dagegen gab es auf den 4 Ackerböden mit niedriger P- Versorgung (Gehaltsklasse $A$ bis $B / C$ ) stets signifikante Mehrerträge durch $\mathrm{CaHPO}_{4}$ - Düngung. In ähnlicher Größenordnung lagen auch die Mehrerträge nach KS Sh. Im Boden Elliehausen übertraf KS Sh sogar die Ca-Phosphat-Variante. Nach KS Gö- Düngung sind die TM- Erträge stärker differenziert. Während auf den Böden Rehburg-Loccum und Elliehausen (beide 
GK A) der Trockenmasseertrag sogar über dem der Ca-Phosphat-Variante lag, war er auf den Böden Börry P-0 und Düshorn (Brache) deutlich geringer. Auf dem Quarzsand war KS Sh dem KS Gö deutlich überlegen.

Die P- Entzüge (Tabelle 7) waren auf dem gut mit P- versorgten Boden Börry P80 trotz P- Düngung in allen Varianten gleich. In den 4 Ackerböden mit GK A bis $\mathrm{B} / \mathrm{C}$ stieg der $\mathrm{P}$ - Entzug nach $\mathrm{CaHPO}_{4}$ - Zufuhr immer deutlich, in 3 Fällen signifikant an. Eine gleiche Wirkung wurde auch mit KS Sh erreicht. Auf dem Boden Elliehausen mit seinem extrem niedrigen P- Gehalt war die P- Wirkung sogar deutlich höher. Klärschlamm Göttingen hatte eine differenziertere Wirkung. Gegenüber der Nullvariante war der P- Entzug nach KS Gö- Zufuhr stets, meist signifikant angestiegen. Aber im Vergleich zur Ca-P-Variante erreichte die Variante KS Gö nur die gleiche Wirkung in den Böden Elliehausen und Rehburg-Loccum.

Tabelle 7: Relative P-Entzüge der Weidelgraspflanzen (Summe von 3 Schnitten) als 3. Kultur nach einer P-Düngung von $187 \mathrm{mg} P / G e f a ̈ ß$ zu Versuchsbeginn

\begin{tabular}{|c|c|c|c|c|c|c|c|c|}
\hline \multirow{2}{*}{$\begin{array}{l}\text { Boden/ } \\
\text { Substrat }\end{array}$} & \multirow[t]{2}{*}{ Standort } & \multirow[t]{2}{*}{$\mathrm{GK}^{1)}$} & \multicolumn{2}{|c|}{ Ohne P } & \multirow{2}{*}{$\begin{array}{c}\mathrm{CaHPO}_{4} \\
\text { relativ }\end{array}$} & \multirow{2}{*}{$\begin{array}{l}\text { KS Gö } \\
\text { relativ }\end{array}$} & \multirow{2}{*}{$\begin{array}{l}\text { KS Sh } \\
\text { relativ }\end{array}$} & \multirow{2}{*}{$\begin{array}{l}G^{1)} \\
5 \%\end{array}$} \\
\hline & & & $\mathrm{mg}$ & relativ & & & & \\
\hline Ton.Schluff & $\begin{array}{l}\text { Börry } \\
\text { P-80 }\end{array}$ & $C / D$ & 182 & 100 & 110 & 105 & 106 & 14 \\
\hline Ton.Schluff & $\begin{array}{l}\text { Börry } \\
\text { P-0 }\end{array}$ & $\mathrm{B} / \mathrm{C}$ & 43 & 100 & 139 & 120 & 141 & 20 \\
\hline Ton.Schluff & $\begin{array}{l}\text { Ellie- } \\
\text { hausen }\end{array}$ & $A$ & 5 & 100 & 183 & 211 & 332 & 37 \\
\hline Lehm.Sand & Rehburg & A & 39 & 100 & 163 & 183 & 164 & 19 \\
\hline Sand & Düshorn & B & 61 & 100 & 147 & 134 & 170 & 41 \\
\hline Quarzsand & $\begin{array}{l}\text { Wefer- } \\
\text { lingen }\end{array}$ & A & --- & --- & $100^{2)}$ & 155 & 251 & 39 \\
\hline \multicolumn{9}{|c|}{ Mittelwert der relativen P-Entzüge: } \\
\hline \multicolumn{4}{|c|}{5 Böden ohne Quarzsand } & --- & 100 & 101 & 123 & \\
\hline \multicolumn{4}{|c|}{$\begin{array}{l}4 \text { Böden ohne Elliehausen und } \\
\text { Quarzsand }\end{array}$} & --- & 100 & 98 & 104 & \\
\hline
\end{tabular}

1) Tukey test, ${ }^{2)}$ absolut $=8,5 \mathrm{mg} \mathrm{P} / \mathrm{Gefäß}$ 
Auf den Böden Börry P-0 und Düshorn (Brache) war die P- Aufnahme deutlich geringer als in der Ca-P- Variante. Auf dem Quarzsand konnte das Weidelgras aber nach KS Gö 55\% und nach KS Sh 151\% mehr P aufnehmen als aus $\mathrm{CaHPO}_{4}$.

Lässt man die Extremvarianten mit Quarzsand und den Boden von Elliehausen (Material des C- Horizontes einer Parabraunerde) außer Betracht, so lagen im 3. Versuchsjahr die mittleren P- Entzüge des Weidelgrases bei allen 3 PQuellen auf nahezu gleichem Niveau (Mittelwerte $\mathrm{CaHPO}_{4}: \mathrm{KS}$ Gö : KS Sh = 100: 98: 104).

\subsubsection{6 Überblick über die P- Entzüge in den drei Versuchsjahren}

Um einen Gesamtüberblick über die P- Entzüge der drei Kulturpflanzen in den drei Versuchsjahren zu gewinnen, werden die P- Entzüge in Tabelle 8 zusammengefasst. Dabei werden aber Relativwerte angegeben, wobei die PEntzüge der $\mathrm{CaHPO}_{4}$ - Variante gleich $100 \%$ gesetzt werden. Damit soll klar werden, wie der Phosphor in den Klärschlämmen relativ zum $\mathrm{P}$ des $\mathrm{CaHPO}_{4}$ wirkt. Es werden auch nur die P- Varianten aufgeführt, in denen $187 \mathrm{mg} \mathrm{P}$ Gefäß ${ }^{-1}$ (30 $\mathrm{mg} \mathrm{P} \mathrm{kg}^{-1}$ Boden) zu Versuchsbeginn angewendet worden sind. Das war die P- Menge, die sich aus den $5 \mathrm{t} \mathrm{TM} \mathrm{ha}^{-1}$ des KS Gö ergeben hatte. Damit werden in der Praxis ca. $60 \mathrm{~kg} \mathrm{P}^{-1}$ ausgebracht. Das ist etwa die PMenge, die durch 3 Ernten abgeführt wird. Tabelle 8 zeigt Folgendes:

Über alle Böden bzw. Substrate hinweg werden bei Anwendung von KS Sh $12 \%$ mehr $\mathrm{P}$ und bei KS Gö $11 \%$ weniger $\mathrm{P}(89 \%)$ als aus $\mathrm{CaHPO}_{4}$ aufgenommen. Das weist auf eine deutliche Differenz in der Ausnutzung des $\mathrm{P}$ bei den zwei Klärschlämmen hin. Bemerkenswert sind die Unterschiede im Verhalten der zwei Klärschlämme auf den verschiedenen Böden. Während alle 3 Kulturpflanzen auf dem tonigen Schluffboden des Standortes Börry unabhängig vom P- Gehalt der 2 Böden aus dem KS Sh nahezu die relativ gleichen P- Mengen wie aus $\mathrm{CaHPO}_{4}$ aufnehmen (95-101\%) sind es aus dem KS Gö nur 83 bzw. $84 \%$. 
Auf dem extrem P- armen kalkhaltigen Löß (Elliehausen) steigen die PAufnahmewerte bei beiden KS vom Mais über Rotklee zum Weidelgras hin an. Aber auch hier wird der Wert der P- Aufnahme aus KS Gö nur im Mittel zu 96\% der aus $\mathrm{CaHPO}_{4}$ erreicht, während es beim KS Sh $137 \%$ sind.

Tabelle 8: Übersicht über die relativen P-Entzüge nach Klärschlammdüngung mit einer P- Gabe von $187 \mathrm{mg} P$ Gefäß ${ }^{-1}$ durch Mais, Rotklee und Weidelgras (WG) auf verschiedenen Böden ( $P$-Entzug nach $\mathrm{CaHPO}_{4}$-Düngung $=100 \%$ )

\begin{tabular}{|c|c|c|c|c|c|c|c|c|c|}
\hline \multirow[t]{2}{*}{$\begin{array}{l}\text { Boden/ } \\
\text { Substrate }\end{array}$} & \multirow[t]{2}{*}{ GK } & \multicolumn{4}{|c|}{$\begin{array}{l}\text { KS Göttingen } \\
\qquad \text { Relativer P-Entzug }\end{array}$} & \multicolumn{4}{|c|}{$\begin{array}{l}\text { KS Salzhausen } \\
\text { Relativer P-Entzug }\end{array}$} \\
\hline & & Mais & Rotklee & WG & Mittel & Mais & Rotklee & WG & Mitte \\
\hline Börry P-80 & $C / D$ & 78 & 76 & 95 & 83 & 90 & 98 & 96 & 95 \\
\hline Börry P-0 & $B / C$ & 82 & 82 & 87 & 84 & 103 & 99 & 101 & 101 \\
\hline Elliehausen & A & 64 & 108 & 116 & 96 & 101 & 128 & 182 & 137 \\
\hline Schluffböden & & 75 & 89 & 99 & 88 & 98 & 108 & 126 & 111 \\
\hline Rehburg & A & 110 & 83 & 113 & 102 & 110 & 106 & 102 & 106 \\
\hline Düshorn & $B$ & 97 & 87 & 91 & 92 & 108 & 98 & 116 & 107 \\
\hline Sandböden & & 104 & 85 & 102 & 97 & 109 & 102 & 109 & 107 \\
\hline Quarzsand & A & 25 & 49 & 155 & 76 & 37 & 90 & 251 & 126 \\
\hline Alle Varianten & & 76 & 81 & 110 & 89 & 92 & 103 & 141 & 112 \\
\hline $\begin{array}{l}\text { Alle Varianten } \\
\text { ohne QS }\end{array}$ & & 86 & 87 & 100 & 91 & 102 & 106 & 119 & 109 \\
\hline $\begin{array}{l}\text { Alle Varianten } \\
\text { ohne QS und } \\
\text { Elliehausen }\end{array}$ & & 92 & 82 & 96 & 90 & 103 & 100 & 104 & 102 \\
\hline
\end{tabular}

Auf den Sandböden Rehburg-Loccum und Düshorn (Brache) werden von beiden KS im Vergleich zu $\mathrm{CaHPO}_{4}$ sehr ähnliche P- Entzüge (KS Gö 97\%, KS Sh 107\%) erreicht, trotzdem übertrifft auch hier der KS Sh auf dem Boden Düshorn den KS Gö im Mittel mit 15\% mehr P- Entzug. Beim Weidelgras sind 
es sogar $24 \%$. Auch auf dem Quarzsand ist der KS Sh dem KS Gö bei jeder Kulturart überlegen. Im Mittel sind aus KS Sh 26\% mehr P als aus $\mathrm{CaHPO}_{4}$ aufgenommen worden, aus KS Gö aber $24 \%$ weniger!

\subsection{Zweijähriges Experiment zur Prüfung der P- Aufnahme der Pflanzen}

\subsubsection{Vorbemerkungen und Versuchsziele}

Im vorausgegangenen Experiment wurden die Klärschlämme bzw. $\mathrm{CaHPO}_{4}$ in die Böden eingebracht und die ersten Pflanzen (Mais) 4 Wochen später ausgesät. In dem nun folgenden Experiment sollte die P- Aufnahme von Pflanzen erst nach längerer Reaktion der P- Dünger mit dem Boden geprüft werden. Die Klärschlämme mit ihren Eisensalz - bzw. $\mathrm{Ca}(\mathrm{OH})_{2}-$ Zugaben enthalten neu gebildete, also wenig gealterte Fe- Verbindungen. Es war zu erwarten, dass sich diese im Boden noch veränderten. Damit könnte sich auch die P- Verfügbarkeit ändern. Um dies zu prüfen, wurde wie folgt vorgegangen: Von den gleichen $\mathrm{KS}$ wie im obigen Experiment sowie von $\mathrm{CaHPO}_{4}$ wurden gestaffelte P- Mengen in die Böden eingemischt (Mitscherlich- Gefäße), diese mit Wasser auf ca. 50\% der maximalen Wasserkapazität eingestellt und in der Klimakammer bei ca. $20^{\circ} \mathrm{C}$ für $1 \mathrm{Jahr}$ inkubiert. Nach dieser Zeit wurden die Böden je eines Mitscherlichgefäßes in 3 Portionen geteilt und in kleinere Gefäße eingefüllt. Diese wurden mit Weidelgras bepflanzt, das dreimal geschnitten wurde. Die P- Aufnahme des Grases sollte zur Beurteilung der PVerfügbarkeit der verschiedenen P- Quellen nach 1- jährigem Umsatz im Boden dienen.

\subsubsection{Versuchsanlage und Durchführung}

Am 20.04.1999 wurde dieser Gefäß-Versuch angesetzt. Auf neun verschiedenen Böden, die sich im verfügbaren Boden $\mathrm{P}$ - Gehalt und im $\mathrm{pH}-$ Wert unterscheiden, wird die Wirkung von zwei eisenhaltigen Klärschlämmen (Tabelle 1) und $\mathrm{CaHPO}_{4}$ untersucht. Die Kenndaten der eingesetzten Böden gibt Tabelle 9 wieder. Es handelt sich um die 5 Böden des ersten Experimentes (Tabelle 2) sowie zusätzlich um 2 lössbürtige tonige Schluffböden aus Dorstadt 
(P-0, P-20) mit niedrigem und hohem P- Gehalt sowie um zwei Sandböden Düshorn P-22 und Hodenhagen P-22 (beide aus dem Raum Walsrode). Sie haben mittlere $\mathrm{P}$ - Gehalte und $\mathrm{pH}$-Werte unter 5.

Tabelle 9: Kenndaten der verwendeten Böden (Korngrößenanteile, $\mathrm{CaCO}_{3}$ Gehalt und Humusgehalt nach Angaben der LUFA Hameln)

\begin{tabular}{|c|c|c|c|c|c|c|c|}
\hline \multirow[t]{2}{*}{ Ort } & Ton & Schluff & Sand & $\mathrm{CaCO}_{3}$ & $\mathrm{pH}$ & Humus & $\mathrm{CAL}^{1)} / \mathrm{DL}^{2)}$ \\
\hline & $\%$ & $\%$ & $\%$ & $\%$ & $\mathrm{CaCl}_{2}$ & $\%$ & $\mathrm{mg} \mathrm{P} / \mathrm{kg}$ \\
\hline Börry P-0 & 20 & 75 & 5 & 1,3 & 7,2 & 2,22 & 46 \\
\hline Börry P-803) & 20 & 75 & 5 & 0,9 & 6,3 & 2,22 & 110 \\
\hline Dorstadt P-0 & 13 & 73 & 14 & 0,7 & 7,0 & 1,89 & 33 \\
\hline Dorstadt P-203) & 13 & 73 & 14 & 0,7 & 7,1 & 1,90 & 98 \\
\hline Elliehausen & 16 & 75 & 9 & 13,3 & 7,7 & 0,50 & 6 \\
\hline Rehb.-Loccum & 8 & 13 & 79 & n.b. & 4,3 & 7,20 & 17 \\
\hline Düshorn & 3 & 13 & 84 & n.b. & 4,9 & 4,00 & 34 \\
\hline \multicolumn{8}{|l|}{ (Brache) } \\
\hline Düshorn P-22 ${ }^{3)}$ & 5 & 11 & 84 & n.b. & 4,9 & 2,20 & 69 \\
\hline Hodenh. P-22 ${ }^{3)}$ & 3 & 6 & 91 & n.b. & 4,7 & 5,50 & 93 \\
\hline
\end{tabular}

\footnotetext{
${ }^{1)}$ bei Lehmböden, ${ }^{2)}$ bei Sandböden, n.b.= nicht bestimmt

3) Jährliche P-Düngung der Parzellen in $\mathrm{kg} \mathrm{ha}^{-1}$
}

Bezugsbasis für die KS- Applikation waren wieder die $5 \mathrm{t} \mathrm{TM}$ ha-1 des KS Göttingen. Damit wurden wie im Kapitel 1.2.2.3 dargelegt $30 \mathrm{mg} \mathrm{P} \mathrm{kg}^{-1}$ Boden angewendet. Hier wurden nun zusätzlich Varianten mit der doppelten Schlammmenge, d.h. auch P- Mengen von $60 \mathrm{mg} \mathrm{P} \mathrm{kg}^{-1}$ und der dreifachen Menge von $90 \mathrm{mg} \mathrm{P} \mathrm{kg}^{-1}$ angelegt. Gleiche $\mathrm{P}$ - Mengen wurden auch vom KS Salzhausen angewendet. Vom $\mathrm{CaHPO}_{4}$ gab es nur die einfache Gabe von 30 $\mathrm{mg} \mathrm{P} \mathrm{kg}{ }^{-1}$, da dessen Löslichkeit als hoch angesehen wurde. Entsprechend den P- Gaben und Fe: P-Verhältnissen der Schlämme wurden folgende Fe-Mengen zugegeben: KS- Gö 157, 314 und $471 \mathrm{mg} \mathrm{Fe}, \mathrm{KS}-\mathrm{Sh}$ 44, 88 und $132 \mathrm{mg} \mathrm{Fe}$ $\mathrm{kg}^{-1}$ Boden. Die Böden $(6 \mathrm{~kg})$ wurden nach Einmischung der Schlämme bzw. 
des $\mathrm{CaHPO}_{4}$ in die Mitscherlichgefäße eingefüllt und inr Wassergehalt auf ca. $50 \%$ der maximalen Wasserkapazität eingestellt. Unter Abdeckung wurden sie 12 Monate bei $20^{\circ} \mathrm{C}$ in einer Klimakammer inkubiert.

Tabelle 10: Versuchsaufbau

Versuchsaufbau Neun Böden (sieh Tab. 9), 3 Wiederholungen

\begin{tabular}{ll}
\hline P- Varianten & (1) ohne P \\
& (2) $\mathrm{CaHPO}_{4}$ \\
& (3) Klärschlamm Göttingen (KS Gö) \\
& (4) Klärschlamm Salzhausen, KS Sh
\end{tabular}

P-Applikationsstufen (1) P0 (ohne P)

(2) $\mathrm{P} 1$ (ca. $30 \mathrm{mg} \mathrm{P} / \mathrm{kg}$ Boden) $=187 \mathrm{mg} \mathrm{P} /$ Gefäß

(3) $\mathrm{P} 2$ (ca. $60 \mathrm{mg} \mathrm{P} / \mathrm{kg}$ Boden) $=374 \mathrm{mg} \mathrm{P} /$ Gefäß

(4) $\mathrm{P} 3$ (ca. $90 \mathrm{mg} \mathrm{P} / \mathrm{kg}$ Boden) $=560 \mathrm{mg} P /$ Gefäß

Am 07.06.2000, also nach 13,5 Monaten, begann ein Vegetationsversuch mit diesen Böden. Um die P- Aufnahme von Pflanzen aus diesen Böden zu prüfen, wurde das Bodenmaterial aus den Mitscherlichgefäßen entnommen, in 3 Portionen von je $1,5 \mathrm{~kg}$ Boden geteilt und in Plastikrohre überführt. Die Rohre hatten einen Durchmesser von 10,5 cm und eine Höhe von $38 \mathrm{~cm}$. Da die 1,5 $\mathrm{kg}$ Boden nicht zur Befüllung der Gefäße ausreichten, wurde der untere Teil der Gefäße mit 2,8 kg Quarzsand befüllt. Vor dem Befüllen der Rohre wurden in die Böden folgende Nährstoffmengen eingearbeitet:

$0,2 \mathrm{~g} \mathrm{~N}$ als $\mathrm{Ca}\left(\mathrm{NO}_{3}\right)_{2} \cdot 4 \mathrm{H}_{2} \mathrm{O}$,

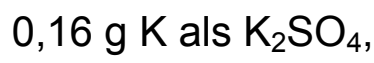

$0,03 \mathrm{~g} \mathrm{Mg}$ als $\mathrm{MgSO}_{4} .7 \mathrm{H}_{2} \mathrm{O}$. 
$150 \mathrm{~g} Q \mathrm{~S}$

$1,5 \mathrm{~kg}$ Boden

$2,8 \mathrm{~kg}$ QS

\section{Befüllung der Gefäße (QS = Quarzsand)}

Die Varianten wurden in dreifacher Wiederholung angesetzt. In diesem Versuch gab es ferner drei Varianten mit den Böden Dorstadt P-0, Rehburg-Loccum und Düshorn (Brache) ohne P- bzw. ohne KS- Düngung aber mit CaO- Gaben, um den möglichen Kalkungseffekt zu erfassen, denn der KS Gö enthält hohe CaOMengen. Es wurden 0,72g CaO pro kg Boden eingesetzt. Am 15.06.2000, also 1 Woche nach der Präparation der Gefäße, erfolgte die Aussaat des Weidelgrases der Sorte LIRASAND (0,05 g pro Gefäß). Die Pflanzen wurden im Drahthaus des Institutes kultiviert. Die Wasserzufuhr erfolgte nicht durch Gießen. Die Gefäße standen in großen Plastikwannen und wurden mit entmineralisiertem Wasser eingestaut (ca. $10 \mathrm{~cm}$ hoch). Dieser Wasserstand wurde alle 2Tage kontrolliert und wenn nötig ergänzt. Diese Wasserversorgung für die Pflanzen erschien ausreichend. Die Pflanzen wurden am 27.07, am 29.08 und am 12.10.2000 geschnitten. Nach dem 1.und 2. Schnitt wurden 0,2 g $\mathrm{N}$ in Form von Ammoniumnitrat nachgedüngt. Anschließend wurden die Trockenmasse und die P- Konzentration in der Trockenmasse bestimmt und der P- Entzug ermittelt.

\subsubsection{Ergebnisse: Sprossmassebildung und P- Entzüge}

Tabelle 11 zeigt die gebildete Sprossmasse als Summe der drei Schnitte. Auf den mit $\mathrm{P}$ hoch versorgten Böden Börry P-80 und Dorstadt P-20 erbrachte die $\mathrm{CaHPO}_{4}$ - Düngung keine Ertragssteigerung. Auf den Böden mit geringerer PVersorgung traten nur auf den zwei Böden mit sehr niedrigem Lactat-P- Gehalt (Rehburg-Loccum, Elliehausen) deutliche Ertragssteigerungen nach PDüngung mit $\mathrm{CaHPO}_{4}$ auf. 
Tabelle 11: Absolut und Relativerträge der Gesamttrockenmasse je Gefäß des Weidelgrases (3 Schnitte)

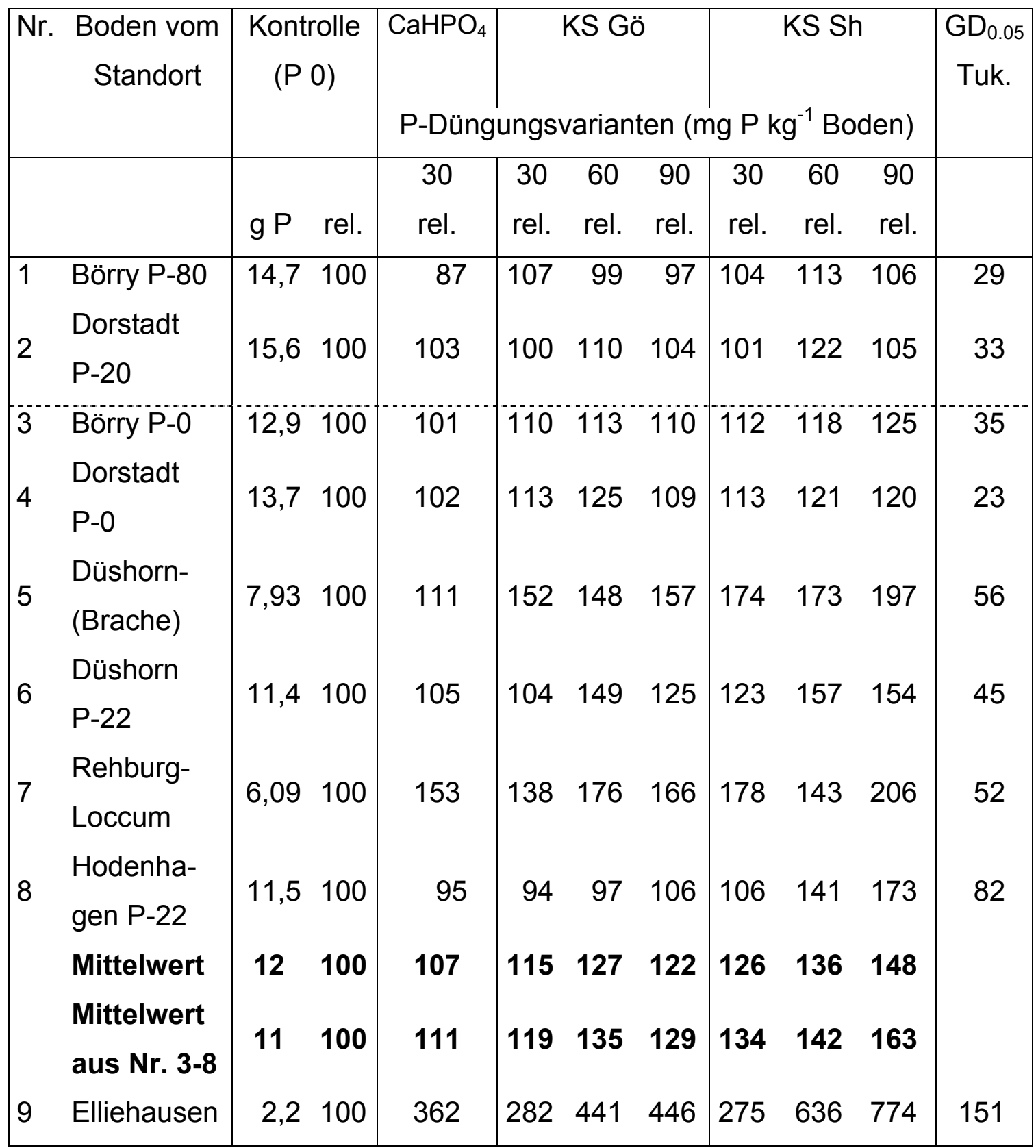

Wichtiger als die Ertragssteigerungen sind die P- Entzüge (Tabelle 12) zur Beurteilung der P- Düngewirkung der Klärschlämme, denn das Wachstum der Pflanzen hängt neben der P-Versorgung von vielen anderen Faktoren ab. 
Tabelle 12: Absolute und relative P-Entzüge je Gefäß des Weidelgrases (3 Schnitte)

\begin{tabular}{|c|c|c|c|c|c|c|c|c|c|c|c|}
\hline \multirow{2}{*}{\multicolumn{2}{|c|}{$\begin{array}{c}\text { Nr. Boden vom } \\
\text { Standort }\end{array}$}} & \multicolumn{2}{|c|}{$\begin{array}{c}\text { Kontrolle } \\
\text { (P 0) }\end{array}$} & $\begin{array}{c}\mathrm{CaHPO}_{4} \\
\text { P-Düng }\end{array}$ & \multicolumn{3}{|c|}{ KS Gö } & \multicolumn{3}{|c|}{ g P kg ${ }^{-1}$ Boden) } & $\mathrm{GD}_{0.05}$ \\
\hline & & $\mathrm{mg} P$ & rel. & $\begin{array}{l}31 \\
\text { rel. }\end{array}$ & $\begin{array}{l}31 \\
\text { rel. }\end{array}$ & $\begin{array}{r}62 \\
\text { rel. }\end{array}$ & $\begin{array}{l}93 \\
\text { rel. }\end{array}$ & $\begin{array}{r}31 \\
\text { rel. }\end{array}$ & $\begin{array}{l}62 \\
\text { rel. }\end{array}$ & $\begin{array}{r}93 \\
\text { rel. }\end{array}$ & \\
\hline 1 & Börry P-80 & 46 & 100 & 101 & 103 & 92 & 95 & 115 & 123 & 128 & 12 \\
\hline 2 & $\begin{array}{l}\text { Dorstadt } \\
\text { P-20 }\end{array}$ & 54 & 100 & 99 & 98 & 99 & 106 & 106 & 118 & 107 & 18 \\
\hline 3 & Börry P-0 & 23 & 100 & 146 & 117 & 129 & 150 & 141 & 173 & 188 & 25 \\
\hline 4 & $\begin{array}{l}\text { Dorstadt } \\
\text { P-O }\end{array}$ & 24 & 100 & 160 & 126 & 138 & 168 & 152 & 206 & 221 & 29 \\
\hline 5 & $\begin{array}{l}\text { Düshorn- } \\
\text { (Brache) }\end{array}$ & 14 & 100 & 122 & 115 & 120 & 111 & 174 & 213 & 307 & 59 \\
\hline 6 & $\begin{array}{l}\text { Düshorn } \\
\text { P-22 }\end{array}$ & 32 & 100 & 118 & 89 & 138 & 139 & 135 & 170 & 192 & 35 \\
\hline 7 & $\begin{array}{l}\text { Rehburg- } \\
\text { Loccum }\end{array}$ & 12 & 100 & 151 & 130 & 146 & 172 & 176 & 200 & 226 & 58 \\
\hline 8 & $\begin{array}{l}\text { Hodenha- } \\
\text { gen P-22 }\end{array}$ & 28 & 100 & 123 & 96 & 102 & 118 & 118 & 164 & 194 & 51 \\
\hline & Mittelwert & 29 & 100 & 128 & 109 & 121 & 132 & 140 & 171 & 195 & \\
\hline & $\begin{array}{l}\text { Mittelwert } \\
\text { aus Nr. 3-8 }\end{array}$ & 22 & 100 & 137 & 112 & 129 & 143 & 149 & 188 & 221 & \\
\hline 9 & Elliehausen & 3 & 100 & 426 & 299 & 338 & 551 & 280 & 683 & 956 & 169 \\
\hline
\end{tabular}

Zunächst ist festzustellen, dass die Graspflanzen auf den 2 Böden Börry P-80 und Dorstadt P-20 nach P-Mineraldüngung mit $\mathrm{CaHPO}_{4}$ nicht mehr Phosphor als aus den Kontrollgefäßen aufgenommen haben. Das war auch nicht unbedingt $\mathrm{zu}$ erwarten, da beide Böden ausreichend mit $\mathrm{P}$ versorgt sind (Tabelle 9), die Pflanzen also im ungedüngten Boden genug $P$ fanden. Die gleiche Aussage gilt auch für den KS Gö und zwar unabhängig von der Höhe 
der P-Zugabe (auf Boden Börry P-80 z.B. 103, 92 bzw. 95\% zur Kontrolle). Interessant ist, dass das Weidelgras nach KS Sh- Düngung auf beiden Böden mehr $\mathrm{P}$ aufgenommen hat als nach $\mathrm{CaHPO}_{4}$-Düngung, signifikant zumindest auf Boden Börry P-80.

Aus diesem Resultat ergibt sich der Schluss, dass nach KS-Anwendung im Vergleich zu einer mineralischen P-Düngerform bei hohem P-Niveau im Boden und gleich hohen P-Zugaben (30 mg P / kg) sowie einem Jahr Einwirkungszeit kein verringerter P-Entzug der Pflanzen auftritt.

Auf allen übrigen Lehmböden, die in ihren Laktatwerten unter $4,6 \mathrm{mg} \mathrm{P} / 100 \mathrm{~g}$ Boden bzw. Sandböden mit Laktatwerten kleiner $9 \mathrm{mg}$ DL-P/100 g Boden liegen, erbringt die Düngung mit $\mathrm{CaHPO}_{4}$ signifikant höhere P-Entzüge von 9 bis $60 \%$. Das heißt, auf diesen Böden tritt eine klare P-Düngewirkung ein, und damit können auch die KS als P-Quelle gut getestet werden. Die Betrachtung der Einzelböden ergibt folgendes:

Boden Börry P-0: Der KS Gö war dem $\mathrm{CaHPO}_{4}$ bei gleicher P-Gabe deutlich unterlegen. Erst bei Applikation der 3-fachen P-Menge (90 $\left.\mathrm{mg} \mathrm{P} \mathrm{kg}^{-1}\right)$ wird der gleiche P-Entzug (150\%) wie mit $\mathrm{CaHPO}_{4}(146 \%)$ erreicht. Der KS Sh ist dagegen dem $\mathrm{CaHPO}_{4}$ nahezu gleichwertig, denn mit der niedrigsten P-Gabe wird fast das Resultat vom $\mathrm{CaHPO}_{4}$ erreicht (141\%).

Boden Dorstadt P-0: Mit der niedrigsten P-Stufe werden mit $\mathrm{CaHPO}_{4} 160 \%$ der P-Aufnahme der Kontrolle (P 0) erreicht, mit KS Gö nur $126 \%$, aber mit KS Sh $152 \%$, also fast das Ergebnis mit $\mathrm{CaHPO}_{4}$.

Die Ergebnisse beider Böden belegen eine fast gleiche Wirkung des KS Sh wie mit $\mathrm{CaHPO}_{4}$, aber eine klare Unterlegenheit des KS Gö.

Auf allen 4 Sandböden Düshorn (Brache), Düshorn P-22, Rehburg und Hodenhagen P-22 ergibt sich stets eine Unterlegenheit des KS Gö gegenüber der P-Mineraldüngerform $\mathrm{CaHPO}_{4}$. Vom KS Gö muss stets die doppelte P-Menge, bei Hodenhagen sogar die 3-fache P-Menge angewendet werden, um den gleichen P-Entzug zu erreichen wie mit der niedrigen P-Gabe 
in Form von $\mathrm{CaHPO}_{4}$. Dagegen wird mit dem KS Sh stets mit der niedrigen PGabe der gleiche bzw. sogar höhere P-Entzug wie mit $\mathrm{CaHPO}_{4}$ erzielt.

\section{Kalkwirkung}

Tabelle 13 vermittelt einen Eindruck von der Wirkung einer Kalkgabe zu drei Böden auf Ertragsbildung und P- Entzüge der Pflanzen.

Tabelle 13: Einfluss einer Kalkung $(0,72 \mathrm{~g} \mathrm{CaO} \mathrm{/} \mathrm{kg}$ Boden) auf den Trockenmasseertrag von Weidelgras (Summe von 3 Schnitten) und den PEntzug von Weidelgras ohne P-Düngung (Mittelwerte von 3 Wiederholungen).

\begin{tabular}{ccccccccc}
\hline Standort & Bodenart & \multicolumn{2}{c}{ Trockenmasse (g) } & \multicolumn{2}{c}{ P-Entzug (mg) } & \multicolumn{2}{c}{$\mathrm{PH}\left(\mathrm{CaCl}_{2}\right)$} \\
& & ohne & mit & ohne & mit & ohne & mit \\
& & Kalk & Kalk & Kalk & Kalk & Kalk & Kalk \\
\hline $\begin{array}{c}\text { Dorstadt } \\
\text { P-0 }\end{array}$ & t. Schluff & $13,7(100)$ & $12,9(94)$ & $24(100)$ & $22(92)$ & 7,0 & 6,8 \\
$\begin{array}{c}\text { Düshorn } \\
\text { Brache }\end{array}$ & Sand & $7,9(100)$ & $8,9(113)$ & $14(100)$ & $13(93)$ & 4,8 & 5,6 \\
$\begin{array}{c}\text { Rehburg- } \\
\text { Loccum }\end{array}$ & Sand & $6,1(100)$ & $9,9(162)$ & $12(100)$ & $12(100)$ & 4,3 & 5,2 \\
\hline
\end{tabular}

Auf dem gut gepufferten tonigen Schluffboden Dorstadt hatte die Kalkzugabe keinen Effekt auf die Pflanzen und das Boden- pH. Auf dem Boden Düshorn stieg das pH zwar von 4,8 auf 5,6 an, aber auch hier war die Auswirkung auf den P- Entzug gering. Das Wachstum bei pH 4,8 wird offenbar nur wenig durch eventuell höhere $\mathrm{Mn}$ - Ionenkonzentrationen beeinflusst. Der Boden RehburgLoccum zeigt einen pH- Anstieg von 4,3 (Al und Mn Toxizität möglich) auf 5,2. Das wirkt sich positiv auf das Wachstum, aber wenig auf die P- Aufnahme aus. Die geringe Anhebung des $\mathrm{pH} 1 \mathrm{Jahr}$ nach der CaO-Zugabe zeigt, dass das zugegebene $\mathrm{CaO}$ - so wie auch das $\mathrm{Ca}(\mathrm{OH})_{2}$ des $\mathrm{KS}$ Gö - während der Inkubation $\mathrm{zu} \mathrm{CaCO}_{3}$ neutralisiert worden sind. 


\section{Zusammenfassend ergibt sich nach 1- jähriger Einbringung der Dünger in die Böden Folgendes:}

Im Mittel der 2 Lehmböden mit niedrigem P-Gehalt ( Börry P-0, Dorstadt P-0 ) und den 4 Sandböden entzogen die Pflanzen bei Düngung mit

$\mathrm{CaHPO}_{4} \quad 137 \% \mathrm{P}$ gegenüber den ungedüngten Varianten,

KS Gö $\quad 112$ \% P gegenüber den ungedüngten Varianten,

KS Sh $\quad 149$ \%P gegenüber den ungedüngten Varianten.

Daraus ergibt sich eine klare Unterlegenheit des KS Göttingen gegenüber dem P-Mineraldünger, während der KS Salzhausen nach 1-jährigem Umsatz im Boden sogar eine bessere P-Anlieferung als $\mathrm{CaHPO}_{4}$ an die Pflanzen gewährleistet.

\subsection{Einjähriges Experiment zur Verfolgung der P- Verfügbarkeit in neun} Böden nach Klärschlammdüngung

\subsubsection{Vorbemerkungen und Versuchsziele}

Pflanzen nehmen nur gelöste Nährstoffe so auch Phosphat auf. Infolge niedriger P- Konzentration in der Bodenlösung liegen nur einige Hundert Gramm P ha ${ }^{-1}$ im Oberboden gelöst vor. Die Pflanzen nehmen aber bei hohen Erträgen bis ca. $30 \mathrm{~kg} \mathrm{P}^{-1}$ auf. D.h., die P-Konzentration der Bodenlösungen muß immer wieder ergänzt werden durch P- Freisetzung von der Festphase. Man spricht von der P- Pufferung. Für die P-Aufnahme der Pflanzen ist also die Orthophosphat $\left(\mathrm{P}_{\mathrm{i}}\right)$-Konzentration der Bodenlösung und die P- Nachlieferung aus der Bodenfestphase wichtig. Gibt man mineralische P-Verbindungen wie $\mathrm{Ca}\left(\mathrm{H}_{2} \mathrm{PO}_{4}\right)_{2}$ oder $\mathrm{CaHPO}_{4}$ in den Boden, so erwartet man einen Anstieg der $\mathrm{P}_{\mathrm{i}}$ - Konzentration bis zur Höhe der Löslichkeit dieser Verbindungen. Für Klärschlämme, wenn sie mit Fe eliminiertes Phosphat enthalten, ist das nicht unbedingt zu erwarten. Es ist sogar denkbar, dass wenig mit $P$ gesättigte FeVerbindungen die P-Sorption in Böden erhöhen. Aus diesen Überlegungen resultierte das Versuchsziel, die Klärschlämme und zum Vergleich $\mathrm{CaHPO}_{4}$ 
in Böden einzubringen, um nach einigen Wochen bzw. Monaten mehrere verschiedene Parameter der P- Verfügbarkeit zu messen:

$\mathrm{P}_{\mathrm{i}}$ - Konzentration der Bodenlösung,

P- Sorption,

pH-Werte der Böden.

Die gefundenen Werte sollen später mit den P- Aufnahmen der Pflanzen in den Experimenten unter 2.2 und 2.3 in Beziehung gesetzt werden, da diese gleichzeitig auf einigen Böden angezogen wurden. In den Experimenten des Kapitel 2.2 waren die Schlämme bzw. $\mathrm{CaHPO}_{4}$ in die Böden eingebracht und die Maissamen 4 Wochen später ausgesät worden. Somit wurde also die PDüngewirkung geprüft, wenn die P- Quellen nur kurzfristig (4 Wochen) Kontakt zum Boden hatten. Die Zeit der Umsetzung der P- Quellen im Boden dürfte aber Auswirkungen auf die P- Verfügbarkeit für die Pflanzen haben. Daraus ergab sich die Frage nach der P-Verfügbarkeit, bei der sich die P- Quellen eine längere Zeit, also z.B. 1 Jahr im Boden umsetzen konnten, bevor die Gefäße bepflanzt wurden. Diese Frage sollte beantwortet werden, indem die Böden mit den zwei Klärschlämmen bzw. $\mathrm{CaHPO}_{4}$ für 1 Monat, 6 Monate bzw. 12 Monate inkubiert und dann auf ihre P- Verfügbarkeit überprüft wurden.

\subsubsection{Versuchsanlage und Durchführung}

Zur Erreichung des Versuchszieles wurde derselbe Versuchsansatz wie in Kapitel 2.3.2 verwendet. Dort ist beschrieben, welche P- Mengen (ca. 30, 60, 90 $\mathrm{mg} \mathrm{P} / \mathrm{kg}$ Boden) in die neun Böden eingebracht wurden und unter welchen Bedingungen sie inkubiert wurden (ca. $50 \%$ max. WK, ca. $20^{\circ} \mathrm{C}$, Klimakammer). Nach einem, 6 und 12 Monaten Inkubationszeit wurden die Gefäße beprobt, ihr Wassergehalt überprüft, wieder abgedeckt und weiter inkubiert.

Anschließend wurden die feuchten Bodenproben direkt zur Gewinnung der Bodenlösung nach Adams (1974) genutzt und deren $\mathrm{P}_{\mathrm{i}}$-Konzentration nach Murphy und Riley (1962) bestimmt. Parallel wurden aliquote Anteile des Bodens 
luftgetrocknet und die P-Sorption mit einer definierten P-Vorlage nach Fritsch und Werner (1989) bestimmt. Die CAL/ DL- P- Gehalte wurden nach Schüller (1969), bzw. Egner et al., (1960) ermittelt. Die pH-Werte wurden in $\mathrm{CaCl}_{2}$ gemessen. Die Methoden sind in Kapitel 2.4 .3 beschrieben. Den Versuchsaufbau und die Probenahme gibt Tabelle 10 wieder.

Es ist bekannt, dass die Al / Fe- Oxidhydrate eine wesentliche Rolle bei der Phosphatsorption spielen (Parfitt 1978). Nach Schwertmann (1964) können mit einer sauren Oxalatlösung die wenig geordneten, amorphen Eisenverbindungen, die eine hohe P- Sorptionsfähigkeit haben, extrahiert werden. Deshalb wurden in mehreren Böden nach der KS- Anwendung diese Fe- Fraktionen bestimmt, um zu sehen, ob ein Zusammenhang zwischen eventuell erhöhter P- Sorption und diesen Eisenverbindungen besteht. Außerdem wurde im Oxalatextrakt auch das darin gelöste Phosphat bestimmt.

\subsubsection{Analysenmethoden in Böden}

\section{Gewinnung der Bodenlösung}

Nach der sogenannten Verdrängungsmethode (Adams, 1974) erfolgte die Gewinnung der Bodenlösung. Dabei wurde frischer feuchter Boden in einen 250 $\mathrm{ml}$ Standzylinder gefüllt, der am Boden eine mit einem Filterpapier abgedeckte Öffnung aufwies. Durch leichtes Aufstauchen des Zylinders wurde der Boden so gelagert, dass keine größeren Hohlräume zu sehen waren. Mit Hilfe einer Schlauchpumpe wurde tropfenweise entmineralisiertes Wasser auf die Bodensäulen getropft. Die Bodenlösung wurde nach unten verdrängt und in Bechergläsern aufgefangen (Lehmböden: 20 ml, Sandböden: $15 \mathrm{ml}$ ).

\section{$P_{i}-$ Konzentration in der Bodenlösung}

Die Bodenlösung wurde durch eine 0,45 $\mu \mathrm{m}$ Membran filtriert, um eine von größeren Kolloiden weitgehend freie Lösung zu erhalten. Nach Murphy und Riley (1962) wurde die quantitative Bestimmung des anorganischen Phosphates in der Bodenlösung durchgeführt. Mit dieser Methode bildet Phosphat in Gegenwart von Molybdat und Kaliumantimonyltartrat einen blauen Farbkomplex. Die Menge des blauen Reaktionsproduktes ist proportional der 
$\mathrm{P}_{\mathrm{i}-}$ Konzentration in der Bodenlösung. Die Messung erfolgte am Spektralphotometer bei $882 \mathrm{~nm}$.

\section{P-Sorptionsvermögen des Bodens}

Um ein Maß für die P- Sorption zu erhalten, wurde die luftgetrocknete Bodenprobe auf $1 \mathrm{~mm}$ gesiebt. $10 \mathrm{~g}$ luftgetrockneter Boden wurden in eine 500 $\mathrm{ml}$ Plastikflasche eingewogen, mit $100 \mathrm{ml} \mathrm{P}$ - Lösung $\left[10 \mathrm{mg} \mathrm{P} \mathrm{L}^{-1}\right.$ als $\mathrm{Ca}\left(\mathrm{H}_{2} \mathrm{PO}_{4}\right)_{2}$ ] versetzt, 2 Stunden auf dem Überkopfschüttler geschüttelt, mit aschefreiem Rundfilter Nr.589 ${ }^{3}$ filtriert. Aus dem Filtrat wurden einige $\mathrm{ml}$ Aliquot zur P- Bestimmung genommen. Nach Murphy und Riley (1962) wurde die PKonzentration der Lösung gemessen. Durch die Differenzbildung von definiert zugegebener und gemessener (nicht gebundener P- Menge) wurde das PSorptionsvermögen des Bodens errechnet.

\section{Bestimmung des oxalatlöslichen Eisens ( $\left.\mathrm{Fe}_{\mathrm{ox}}\right)$ im Boden}

Nach Schwertmann (1964) wurde das oxalatlösliche Fe bestimmt. Dazu wurden $2 \mathrm{~g}$ luftgetrockneter Boden (1mm gesiebt) mit $100 \mathrm{ml}$ eines Gemisches aus $0,2 \mathrm{~mol} \mathrm{NH}_{4}$ - Oxalat und $0,2 \mathrm{~mol}$ Oxalsäure $(\mathrm{pH} 3)$ versetzt, 2 Stunden auf dem Überkopfschüttler im Dunkelraum geschüttelt und mit aschefreiem Filterpapier filtriert. Im Filtrat wurde die Fe- und P-Bestimmung mittels ICP vorgenommen. Die Analysen führte das Institut für Geochemie der Universität Göttingen durch.

\section{Phosphatgehalt des Bodens (CAL-P / DL- P)}

Nach Schüller (1969) wurde mit der Calcium- Acetat- Lactat- Methode der pflanzenverfügbare Phosphatgehalt in den Lehmböden bestimmt. Dazu wurden $5 \mathrm{~g}$ luftgetrockneter Boden (2 mm gesiebt) mit 100ml CAL- Gebrauchslösung $(\mathrm{pH} 4,1)$ und einer Spatelspitze Aktivkohle versetzt, 2 Stunden auf dem Horizontalschüttler geschüttelt und durch 5951/2 Faltenfilter filtriert. Aus dem Filtrat wurden einige $\mathrm{ml}$ Aliquot zur P- Bestimmung abpipettiert und der PGehalt nach Murphy und Riley (1962) bestimmt. In den Sandböden wurde nach Egner et al., (1960) mit der Doppellaktat- Methode der pflanzenverfügbare Phosphatgehalt bestimmt. Dazu wurden $5 \mathrm{~g}$ luftgetrockneter Boden $(2 \mathrm{~mm}$ gesiebt) mit 250ml DL- Gebrauchslösung (pH 3,6) versetzt, 1 1/2 Stunden auf 
dem Überkopfschüttler geschüttelt und mit 5951/2 Faltenfilter filtriert. Aus dem Filtrat wurden einige $\mathrm{ml}$ Aliquot zur P- Bestimmung abpipettiert und darin der PGehalt nach Murphy und Riley (1962) bestimmt.

\section{pH-Wert Bestimmung}

Zur Bestimmung der pH-Werte wurde eine Bodensuspension aus $10 \mathrm{~g}$ luftgetrocknetem Boden und $25 \mathrm{ml} \mathrm{0,01} \mathrm{mol} \mathrm{CaCl}_{2}$ - Lösung hergestellt und mit einem Glasstab mehrmals umgerührt. Nach 30 Minuten erfolgte die $\mathrm{pH}$ Wertmessung mit einer Einstabmesselektrode.

\subsubsection{Ergebnisse des Inkubationsversuches zur Verfolgung der P-Verfügbarkeit über 12 Monate}

\subsubsection{Orthophosphatkonzentrationen ( $P_{\mathrm{i}^{-}}$Konzentration) in den Bodenlösungen}

Tabelle 14 und 15 zeigten die $\mathrm{P}_{\mathrm{i}}$-Werte der Bodenlösungen. Wie erwartet stiegen durch die $\mathrm{Ca}\left(\mathrm{H}_{2} \mathrm{PO}_{4}\right)_{2}$-Düngung die Werte nach einem Monat an, in den Schluffböden im Mittel im Vergleich zur Kontrolle auf 198 \% (Tabelle 14) und in den Sandböden auf $232 \%$ (Tabelle 15). Mit dem KS Sh wurden nur Werte von 124 bzw. 113 \% erreicht, mit den KS Gö sanken die Werte im Mittel auf 82 bzw. $62 \%$ ab. Auf den Böden Börry P-80, Dorstadt P-20, Düshorn P-22 und Hodenhagen P-22 sind die Absenkungen unter den Ausgangswert durch KS Gö statistisch signifikant. Das sind die Böden, die die höheren P- Ausgangswerte (vgl. Tabelle 9) besaßen. Dagegen stiegen auf den Böden mit den niedrigen PAusgangsgehalten (Elliehausen, Börry P-0, Dorstadt P-0, Rehburg-Loccum) die $\mathrm{P}_{\mathrm{i}}$ - Werte an, bei den ersten drei sogar signifikant. Der Einfluß der selben PGabe als KS Gö auf die $\mathrm{P}_{\mathrm{i}}$ - Konzentration hing also vom P- Gehalt des Bodens ab. Dieses Bild bestätigt sich prinzipiell auch nach 6 bzw. 12 Monaten. Wichtig erscheint, dass der Unterschied in der Wirkung der zwei Klärschlämme nach 12 Monaten noch immer klar zu erkennen ist. Während $\mathbf{K S}$ Sh die $\mathbf{P}_{\mathbf{i}}$ - Werte in allen neun Böden im Vergleich zur $P$ 0 anhebt, besonders deutlich in den Lehmböden, wird die $\mathrm{P}_{\mathrm{i}}$ - Konzentration durch KS Gö in den Böden Börry P80, Dorstadt P-20, Düshorn P-22 und Hodenhagen P- 22 deutlich gesenkt. 
Tabelle 14: Orthophosphatkonzentration $\left(P_{i}\right)$ in der Bodenlösung $\left(\mu g \Gamma^{-1}\right)$ von 5 Schluffböden nach Inkubation von 1, 6 bzw. 12 Monaten, P-Zugabe: 30mg P kg $\mathrm{kg}^{-1}$ Boden entsprechend $187 \mathrm{mg} P$ / Gefäß

\begin{tabular}{|c|c|c|c|c|c|}
\hline Standort & Ohne P & $\mathrm{CaHPO}_{4}$ & KS Gö & KS Sh & $\mathrm{GD}_{0,05}$ (Tukey) \\
\hline \multicolumn{6}{|c|}{--1.-- } \\
\hline & & Nach $1 \mathrm{Mc}$ & Filtration) & & \\
\hline Elliehausen & 4 & 71 & 17 & 13 & 19 \\
\hline Börry P-0 & 56 & 202 & 79 & 81 & 21 \\
\hline Dorstadt P-0 & 60 & 231 & 98 & 147 & 20 \\
\hline Börry P-80 & 279 & 800 & 181 & 355 & 33 \\
\hline Dorstadt P-20 & 713 & 890 & 536 & 781 & 26 \\
\hline Mittelwert (rel.) & $222(100)$ & $438(198)$ & $182(82)$ & 275 (124) & \\
\hline \multicolumn{6}{|c|}{ Nach 6 Monaten (mit Filtration 0,45 $\mu \mathrm{m}$ ) } \\
\hline Elliehausen & 5 & 46 & 11 & 9 & 7 \\
\hline Börry P-0 & 58 & 176 & 95 & 119 & 16 \\
\hline Dorstadt P-0 & 71 & 313 & 120 & 207 & 38 \\
\hline Börry P-80 & 311 & 576 & 232 & 448 & 16 \\
\hline Dorstadt P-20 & 748 & 1016 & 643 & 938 & 38 \\
\hline Mittelwert (rel.) & $238(100)$ & $425(179)$ & $220(92)$ & 344 (144) & \\
\hline \multicolumn{6}{|c|}{ Nach 12 Monaten (mit Filtration $0,45 \mu \mathrm{m}$ ) } \\
\hline Elliehausen & 4 & 45 & 14 & 10 & 2 \\
\hline Börry P-0 & 78 & 250 & 110 & 160 & 22 \\
\hline Dorstadt P-0 & 78 & 354 & 154 & 264 & 12 \\
\hline Börry P-80 & 385 & 692 & 273 & 615 & 28 \\
\hline Dorstadt P-20 & 728 & 1017 & 698 & 1012 & 40 \\
\hline Mittelwert (rel.) & $255(100)$ & $472(185)$ & $250(98)$ & 412 (162) & \\
\hline
\end{tabular}


Tabelle 15: Orthophosphatkonzentration $\left(P_{i}\right)$ in der Bodenlösung $\left(\mu \mathrm{I}^{-1}\right)$ von 4 Sandböden nach Inkubation von 1, 6 bzw. 12 Monaten, P-Zugabe: 30mg P kg-1 Boden entsprechend $187 \mathrm{mg} P$ / Gefäß

\begin{tabular}{|c|c|c|c|c|c|}
\hline Standort & Ohne P & $\mathrm{CaHPO}_{4}$ & KS Gö & KS Sh & $\mathrm{GD}_{0,05}$ (Tukey) \\
\hline \multicolumn{6}{|c|}{ 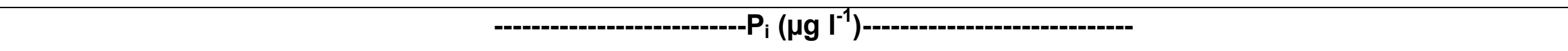 } \\
\hline \multicolumn{6}{|c|}{ Nach 1 Monat (ohne Filtration) } \\
\hline Rehburg-Loccum & 11 & 52 & 20 & 38 & 26 \\
\hline Düshorn (Brache) & 41 & 86 & 48 & 54 & 14 \\
\hline Düshorn P-22 & 276 & 677 & 131 & 293 & 70 \\
\hline Hodenhagen P-22 & 142 & 283 & 94 & 148 & 20 \\
\hline Mittelwert (rel.) & $118(100)$ & $275(232)$ & $73(62)$ & $133(113)$ & \\
\hline \multicolumn{6}{|c|}{ Nach 6 Monaten (mit Filtration $0,45 \mu \mathrm{m}$ ) } \\
\hline Rehburg-Loccum & 14 & 32 & 16 & 25 & 5 \\
\hline Düshorn (Brache) & 21 & 43 & 21 & 43 & 9 \\
\hline Düshorn P-22 & 245 & 499 & 147 & 271 & 9 \\
\hline Hodenhagen P-22 & 111 & 183 & 77 & 123 & 15 \\
\hline Mittelwert (rel.) & $98(100)$ & $189(193)$ & $65(66)$ & $115(117)$ & \\
\hline \multicolumn{6}{|c|}{ Nach 12 Monaten (mit Filtration $0,45 \mu \mathrm{m}$ ) } \\
\hline Rehburg-Loccum & 13 & 28 & 10 & 26 & 5 \\
\hline Düshorn (Brache) & 19 & 34 & 19 & 33 & 4 \\
\hline Düshorn P-22 & 280 & 477 & 177 & 299 & 19 \\
\hline Hodenhagen P-22 & 118 & 164 & 85 & 121 & 9 \\
\hline Mittelwert (rel.) & $108(100)$ & $176(163)$ & $73(68)$ & $120(111)$ & \\
\hline
\end{tabular}




\subsubsection{P-Sorption der Böden}

Aus Tabelle16 und 17 ergibt sich durch die Zufuhr von $\mathrm{CaHPO}_{4}$ nach 1 Monat wie erwartet eine Abnahme der P-Sorption bei den Schluffböden auf $81 \%$ (Tabelle16), den Sandböden auf 91\% (Tabelle17). Durch die Zufuhr von KS Sh blieb die P-Sorption nahezu auf dem gleichen Niveau wie bei den Kontrollen (im Mittel 95\% bzw. 103\%). Von 8 Böden stieg nach Zugabe von KS Gö die PSorption in 7 Fällen (nach 1 Monat) signifikant an. Auf den Lehmböden waren es im Mittel ca. 16 \% und auf den Sandböden ca. 12 \%. Auch nach 6 bzw. 12 Monaten waren die Trends die gleichen.

In der Tabelle18 kann neben der Wirkung der Zeit auf die P- Sorption auch die Wirkung der Klärschlammmenge auf die P- Sorption abgelesen werden, da neben der P- Menge von $30 \mathrm{mg} \mathrm{kg}^{-1}$ auch die doppelte bzw. dreifache KSMenge in die Böden eingebracht worden war (60 bzw. $90 \mathrm{mg} \mathrm{P} \mathrm{kg}^{-1}$ ). Betrachtet man zunächst den Faktor Zeit und setzt die P-Sorption bei den drei P-Stufen nach 1 Monat jeweils gleich 100, so sieht man, dass die Werte innerhalb von 12 Monaten auf den Schluffböden bei KS Gö je nach KS- Zugabe auf 91, 88 bzw. $89 \%$ also im Mittel um ca. 10\% absinken. Auf den Sandböden sind es im Mittel innerhalb von 12 Monaten 91, 83 bzw. 78\%, also im Mittel ca. 16\%. Nach KS Sh- Düngung ist ein ähnlicher Trend zu beobachten: Absenkung auf den Schluffböden im Mittel um ca. 10\%, auf den Sandböden ca. 7\%. Die Veränderungen in den einzelnen Böden sind in den Tabellen 8.4 und 8.5 im Anhang zu finden.

Als mögliche Ursache für diese Senkung der P- Sorption kommt eine Alterung der Eisenverbindungen in Frage. Das ist später zu diskutieren.

Betrachtet man den Effekt der angewendeten P- Mengen in Form der Klärschlämme, so zeigt sich Folgendes:

Auf den Schluffböden steigt zu allen drei Untersuchungszeitpunkten die PSorption nach KS Gö- Anwendung von der niedrigen P- Gabe bis zur höchsten P- Gabe ganz klar auf etwa $120 \%$ an (118 bis 123\%). Bei den Varianten mit KS Sh liegen die Sorptionswerte stets unter 100\% (87 - 94\%), d.h. selbst eine 
Verdreifachung der KS Sh- Menge erhöhte zu keiner Zeit die Relativwerte der P-Sorption.

Auf den Sandböden ist das Bild anders. Hier zeigten die Böden nur nach einem Monat einen klaren Trend zum Anstieg der P-Sorption nach erhöhter PZufuhr mit KS Gö: $100<114<127 \%$. Nach 12 Monaten war der Anstieg viel schwächer: $100<103<108 \%$. Die Einzelwerte in Tabelle 8.4 und $8.5 \mathrm{im}$ Anhang belegen, dass sich die einzelnen Böden etwas differenzierter verhalten. Nach 12 Monaten zeigten die Böden Düshorn P-22 und Hodenhagen P-22 noch einen klaren Anstieg der P-Sorption mit der Klärschlammmenge, während bei Düshorn (Brache) und Rehburg-Loccum die Werte zwischen den P- Stufen sehr ähnlich waren (99 bis 105\%).

Die P- Gabe in Form von KS Sh hatte zu keiner Zeit einen eindeutigen Einfluß auf die Werte der P-Sorption. Die Werte lagen zwischen 95 und 106\% bezogen auf die niedrigste P-Stufe. 
Tabelle 16: P-Sorption von 5 Schluffböden 1 Monat bzw. 6 Monate und 12 Monate nach der P-Düngung,

$30 \mathrm{mg} P$ / kg Boden entsprechend $187 \mathrm{mg} P$ / Gefäß

\begin{tabular}{|c|c|c|c|c|c|}
\hline Standort & Ohne P & $\mathrm{CaHPO}_{4}$ & KS Gö & KS Sh & $\mathrm{GD}_{0,05}$ (Tukey) \\
\hline \multicolumn{6}{|c|}{ 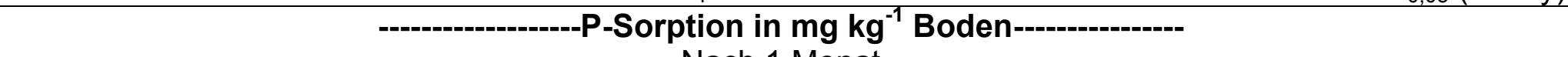 } \\
\hline \multicolumn{6}{|c|}{ Nach 1 Monat } \\
\hline Elliehausen & 66 & 48 & 67 & 53 & 11,0 \\
\hline Börry P-0 & 43 & 38 & 51 & 44 & 1,9 \\
\hline Dorstadt P-0 & 49 & 41 & 54 & 46 & 1,4 \\
\hline Börry P-80 & 27 & 24 & 43 & 33 & 3,0 \\
\hline Dorstadt P-20 & 28 & 25 & 35 & 27 & 0,8 \\
\hline Mittelwert (rel.) & $43(100)$ & $35(81)$ & $50(116)$ & $41(95)$ & \\
\hline \multicolumn{6}{|c|}{ Nach 6 Monaten } \\
\hline Elliehausen & 57 & 52 & 59 & 57 & \\
\hline Börry P-0 & 36 & 30 & 45 & 39 & 4,7 \\
\hline Dorstadt P-0 & 45 & 38 & 48 & 42 & 4,0 \\
\hline Börry P-80 & 27 & 22 & 41 & 25 & 2,3 \\
\hline Dorstadt P-20 & 30 & 25 & 36 & 29 & 6,0 \\
\hline Mittelwert (rel.) & $39(100)$ & $33(85)$ & $46(118)$ & $38(97)$ & \\
\hline \multicolumn{6}{|c|}{ Nach 12 Monaten } \\
\hline Elliehausen & 58 & 53 & 60 & 58 & 1,6 \\
\hline Börry P-0 & 42 & 37 & 48 & 42 & 3,6 \\
\hline Dorstadt P-0 & 41 & 34 & 45 & 37 & 8,1 \\
\hline Börry P-80 & 30 & 23 & 41 & 28 & 3,2 \\
\hline Dorstadt P-20 & 27 & 24 & 30 & 26 & 5,6 \\
\hline Mittelwert (rel.) & $40(100)$ & $34(85)$ & 45 (113) & $38(95)$ & \\
\hline
\end{tabular}


Tabelle 17: P-Sorption von 4 Sandböden 1 Monat bzw. 6 Monate und 12 Monate nach der P-Düngung,

$30 \mathrm{mg} P$ / kg Boden entsprechend $187 \mathrm{mg} P$ / Gefäß

\begin{tabular}{|c|c|c|c|c|c|}
\hline Standort & Ohne P & $\mathrm{CaHPO}_{4}$ & KS Gö & KS Sh & $\mathrm{GD}_{0,05}$ (Tukey) \\
\hline \multirow{2}{*}{\multicolumn{6}{|c|}{$\begin{array}{c}\text {----------P-Sorption in } \mathbf{~ m g ~ k g}^{-1} \text { Boden-------- } \\
\text { Nach } 1 \text { Monat }\end{array}$}} \\
\hline & & & & & \\
\hline Rehburg-Loccum & 82 & 78 & 83 & 81 & 2,6 \\
\hline Düshorn (Brache) & 72 & 66 & 78 & 73 & 4,6 \\
\hline Düshorn P-22 & 27 & 23 & 38 & 31 & 4,4 \\
\hline Hodenhagen P-22 & 53 & 43 & 60 & 54 & 5,5 \\
\hline Mittelwert (rel.) & $58(100)$ & $53(91)$ & 65 (112) & $60(103)$ & \\
\hline \multicolumn{6}{|c|}{ Nach 6 Monaten } \\
\hline Rehburg-Loccum & 88 & 80 & 84 & 85 & 4,5 \\
\hline Düshorn (Brache) & 83 & 78 & 82 & 82 & 2,6 \\
\hline Düshorn P-22 & 54 & 47 & 57 & 53 & 5,4 \\
\hline Hodenhagen P-22 & 61 & 54 & 82 & 69 & 3,7 \\
\hline Mittelwert (rel.) & $71(100)$ & $64(90)$ & 76 (107) & $72(101)$ & \\
\hline \multicolumn{6}{|c|}{ Nach 12 Monaten } \\
\hline Rehburg-Loccum & 85 & 83 & 87 & 85 & 9,1 \\
\hline Düshorn (Brache) & 77 & 70 & 79 & 75 & 5,9 \\
\hline Düshorn P-22 & 33 & 27 & 38 & 33 & 5,9 \\
\hline Hodenhagen P-22 & 56 & 53 & 57 & 60 & 3,2 \\
\hline Mittelwert (rel.) & $63(100)$ & $58(92)$ & $65(103)$ & $63(100)$ & \\
\hline
\end{tabular}


Tabelle 18: Einfluss von Klärschlamm (Höhe der P-Gabe) und Inkubationszeit (1, 6 und 12 Monate) auf die relative P-Sorption der Böden (P-Sorption der Böden ohne $P=100$ )

Schluffböden: Mittel aus Börry P - 0, P - 80; Dorstadt P-0, P-20

Sandböden: Mittel aus Düshorn, Rehburg, Hodenhagen

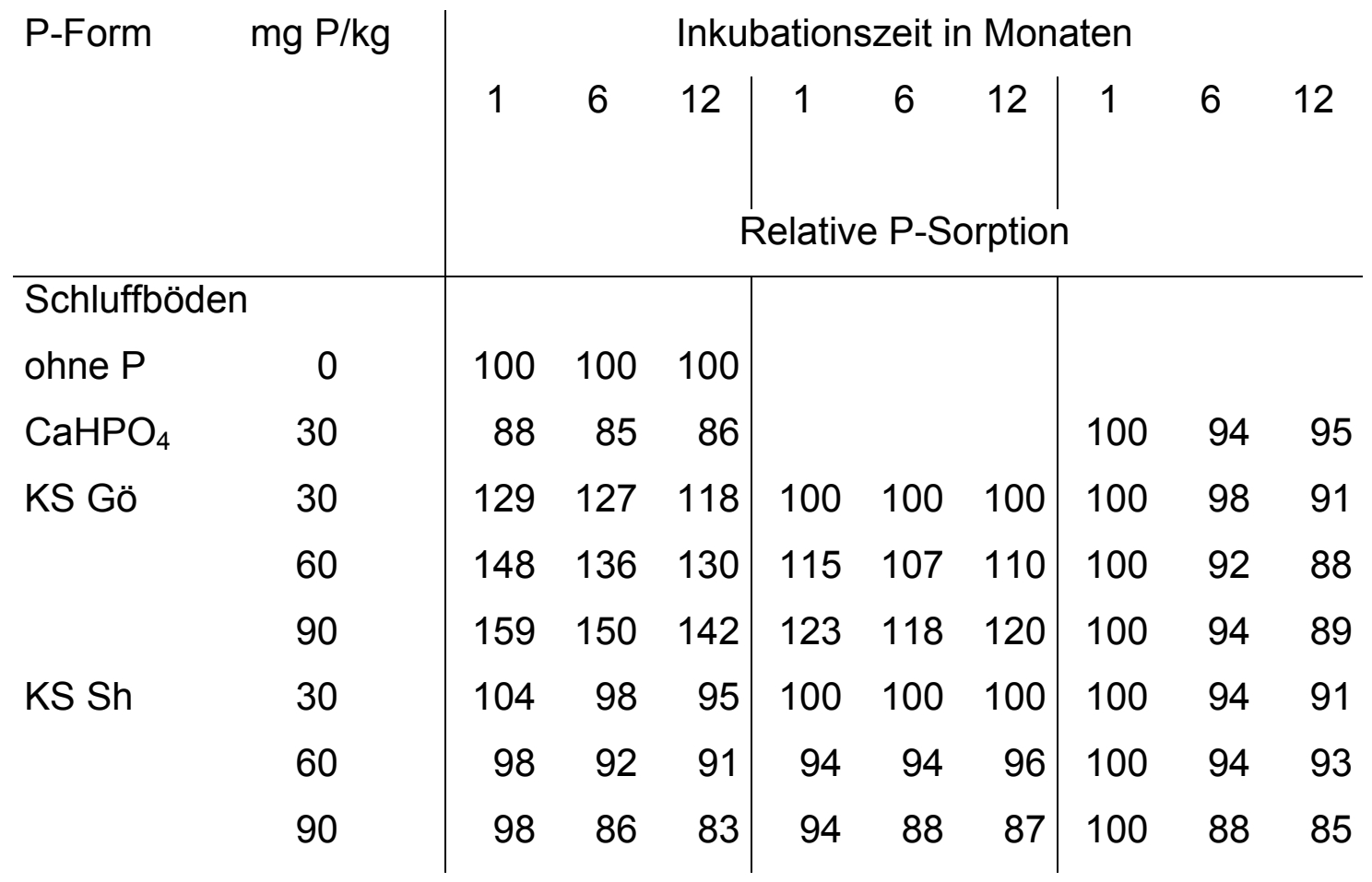

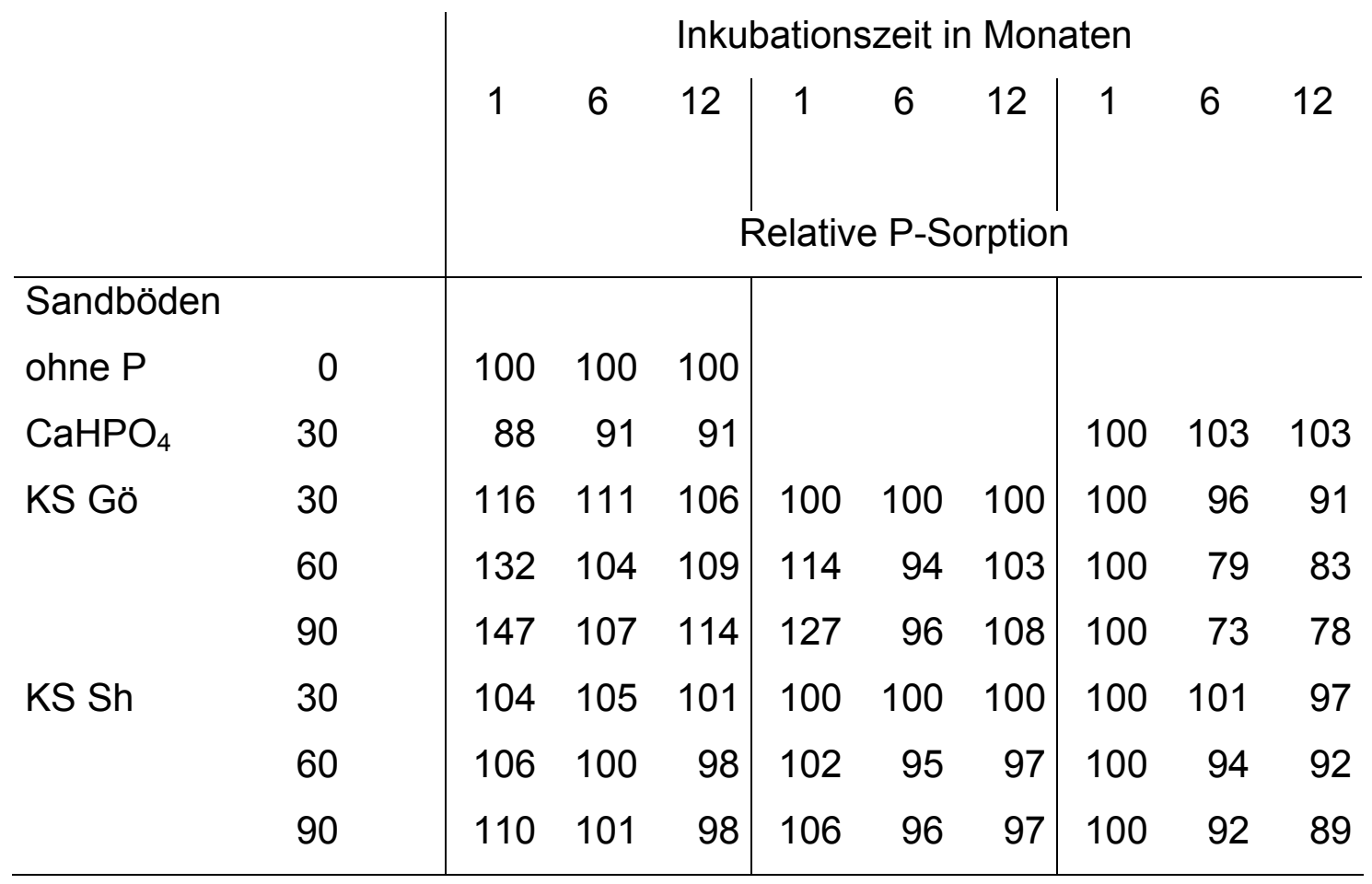




\subsubsection{Beziehung zwischen den Bodengehalten an Feox und der P- Sorption}

Die Abbildungen 7, 8 und 9 zeigen die Zusammenhänge zwischen den Gehalten der Böden an oxalatlöslichem Eisen $\left(\mathrm{Fe}_{\mathrm{ox}}\right)$ und der P-Sorption. Wiedergegeben sind stets die Messwerte, die einen Monat nach der Klärschlammapplikation in den Böden für $\left(\mathrm{Fe}_{\mathrm{ox}}\right)$ und die P-Sorption gefunden wurden. P-0 entspricht der Variante ohne P- Düngung. Gö1, Gö2 und Gö3 stehen für die gestaffelten P- Gaben (187, 374, 560 mg P / Gefäß bzw. 30, 60 und $90 \mathrm{mg} \mathrm{P} \mathrm{/} \mathrm{kg} \mathrm{Boden).} \mathrm{Vom} \mathrm{KS} \mathrm{Sh} \mathrm{wurde} \mathrm{nur} \mathrm{der} \mathrm{Wert} \mathrm{mit} \mathrm{der} \mathrm{höchsten} \mathrm{P-}$ Gabe eingezeichnet, da nur dort eine interessante Fe- Menge mit ausgebracht worden war. Diese lag in der Größenordnung der Gö1- Gabe. Ausnahmslos steigt die $\mathrm{P}$ - Sorption mit zunehmenden $\mathrm{Fe}_{\mathrm{ox}}-$ Werten an. Dabei liegen die Bestimmtheitsmaße für die Regressionsgeraden bei den Lehmböden zwischen 0,87 und 0,58 aber bei den Sandböden (Abbildung 9) über 0,92. Das bedeutet, dass die Beziehung zwischen den $\mathrm{Fe}_{\mathrm{ox}}-\mathrm{Gehalten}$ in den Böden und der PSorption eng ist.

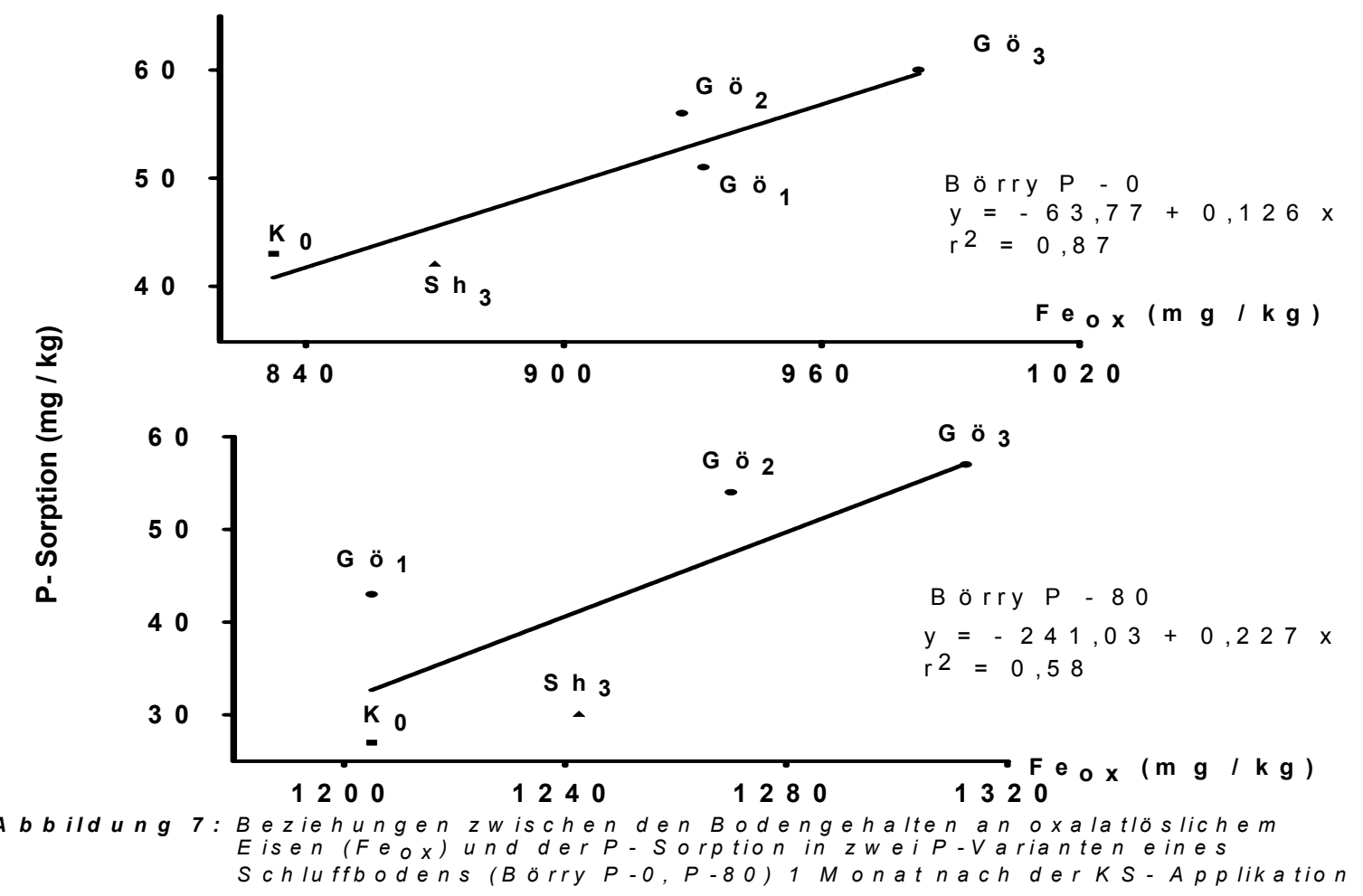




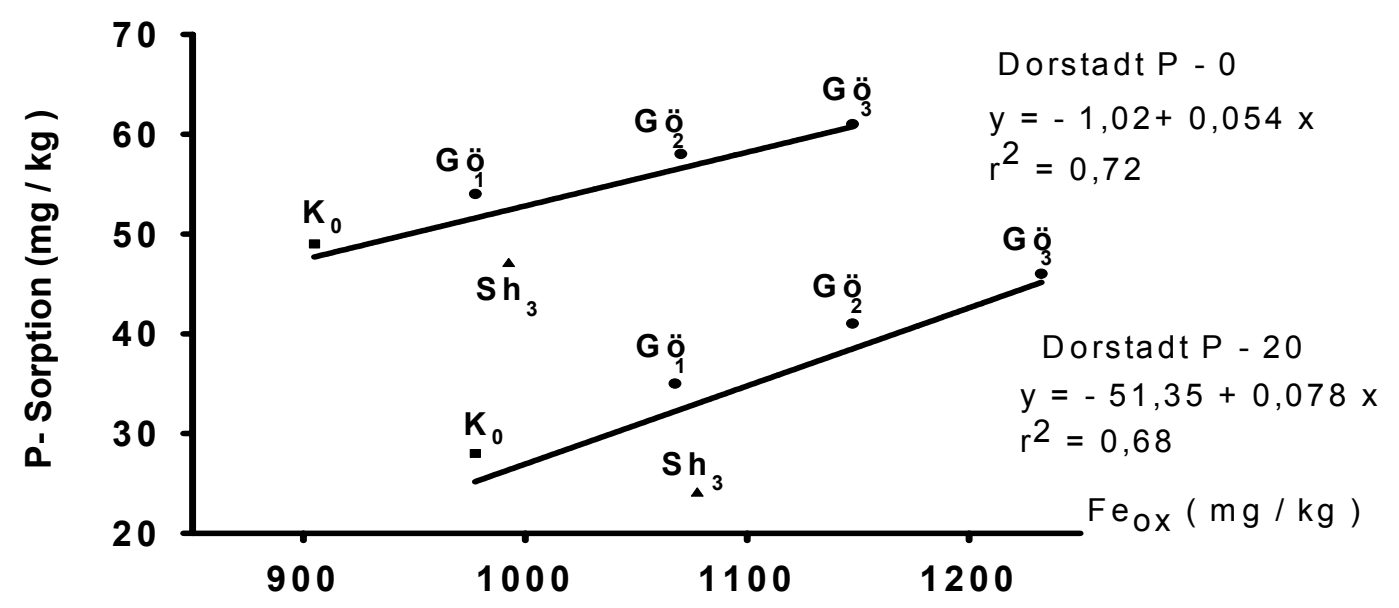

Abbildung 8: Beziehungen zwischen den Bodengehalten an oxalatlöslichem Eisen $\left(F e_{O X}\right)$ und der $P$ - Sorption in ywei Varianten des Lehmbodens Dorstadt (P-O, P-20) 1 Monat nach der KS-Applikation

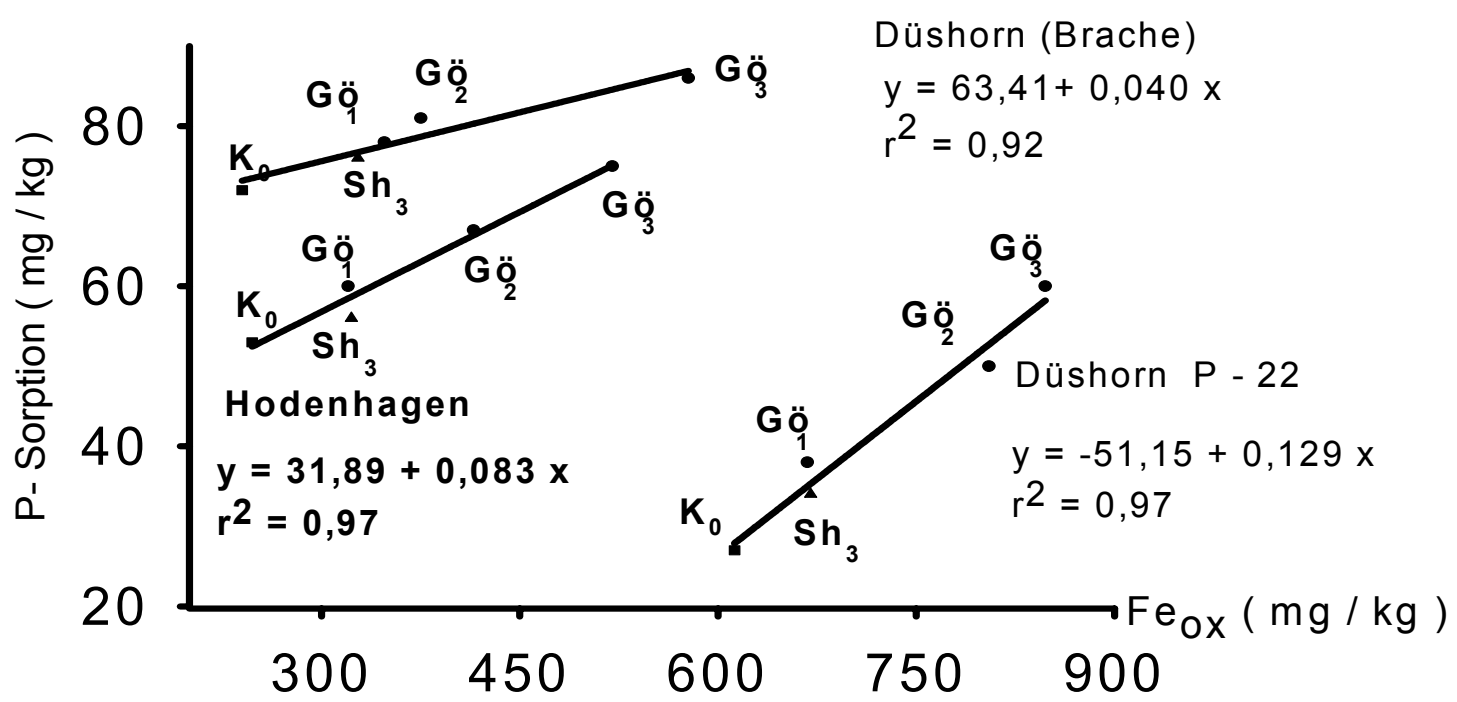

Abbildung 9: Beziehungen zwischen den Bodengehalten an oxalatlöslichem Eisen (Feox) und der P-Sorption in drei Sandböden Düshorn (Brache), Düshorn P-22, Hodenhagen P-22) 1Monat nach der KS-Applikation 


\subsection{Diskussion und Schlussfolgerungen}

Die zentrale Frage der Untersuchungen war, ob der hohe Fe-Gehalt des KS Gö bzw. dessen weites molares Fe: $P$ - Verhältnis von 1:0,3 negative Auswirkungen auf die P-Aufnahme und damit das Wachstum der Pflanzen hat und welche Ursachen dafür verantwortlich sind. Als Zweites ergab sich die Frage, ob sich die P-Aufnahme der Pflanzen aus den mit verschiedenen PQuellen gedüngten Böden längerfristig, hier im Laufe von 3 Jahren, zwischen den 3 P-Düngungsvarianten veränderte.

Berechnet man zur Beantwortung der ersten Frage zunächst die Mittelwerte der P-Aufnahmen des Maises für die Varianten mit $187 \mathrm{mg} \mathrm{P} \mathrm{/} \mathrm{Gefäß} \mathrm{(} \mathrm{ca.} \mathrm{optimale}$ $\mathrm{P}$ - Gabe), so konnte der Mais im Vergleich zur $\mathrm{CaHPO}_{4}-$ Variante aus den 5 Böden sowie dem Quarzsand, die mit KS Gö gedüngt waren, nur $76 \% \mathrm{P}$ aufnehmen, während aus den Böden mit KS Sh 92 \% P aufgenommen werden konnten (Tabelle 8). Pauschal betrachtet lagen also beide Klärschlämme in ihrer P-Bereitstellung für die Pflanzen klar unter derjenigen der OrthophosphatVariante, wobei der KS Gö durch die niedrigsten Werte besonders auffiel. Eine detailliertere Betrachtung der Resultate ergibt ein differenzierteres Bild von der Wirkung der zwei Schlämme. Die Resultate mit dem Substrat Quarzsand sind in erster Linie für die niedrigen Mittelwerte der P-Entzüge der Mais- Pflanzen verantwortlich ( 25 bzw. 37 \%). Einerseits ist der P- freie Quarzsand ein ideales Substrat, um die Aufnehmbarkeit gelösten Phosphates zu prüfen, da er keine PSorptionsplätze besitzt und damit die P-Verfügbarkeit nicht negativ beeinflussen kann. Andererseits ist er ein inertes Material und kein Boden mit all seinen biologischen und biochemischen Eigenschaften. Die Ausklammerung der Quarzsandvarianten aus der Betrachtung erhöht die Werte der P-Aufnahmen gegenüber $\mathrm{CaHPO}_{4}$ bei KS Gö auf $86 \%$ bzw. $102 \%$ bei KS Sh (Tabelle 8 ). Das heißt, bei Anwendung von KS Sh wurde im Mittel aller Ackerböden, bei geringen Unterschieden zwischen ihnen, die gleiche P-Aufnahme erreicht wie aus $\mathrm{CaHPO}_{4}$.

Bemerkenswert ist, dass sich die $86 \%$-ige P-Aufnahme aus den KS GöVarianten bei Trennung der Schluff- von den Sandböden weiter stark differenziert. Auf den Schluffböden wurde nur eine P-Aufnahme von $75 \%$ 
erreicht, bei den Sandböden aber im Mittel $104 \%$, also Gleichheit mit den $\mathrm{CaHPO}_{4}$-Varianten erzielt. Auf dieses Resultat ist später zurückzukommen.

Beim gleichen P- Düngungsniveau verhält sich Rotklee ähnlich wie Mais. Auch er nahm im Mittel aller 5 Böden und Quarzsand aus KS Gö nur 81\% P im Vergleich zur $\mathrm{CaHPO}_{4}$ - Variante auf, während aus den Böden mit KS Sh 103\% aufgenommen wurden. Läßt man auch hier die Quarzsandvariante weg, so steigen die P- Entzüge bei KS Gö auf $87 \%$ bei KS Sh auf $106 \%$. Eine Trennung in Schluff- und Sandböden brachte beim Rotklee keine so deutlichen Unterschiede wie beim Mais. Vom Boden Elliehausen abgesehen, war die PAufnahme des Rotklee aus KS Gö auf allen Böden der Variante mit $\mathrm{CaHPO}_{4}$ und der KS Sh- Variante deutlich unterlegen.

Das Weidelgras als dritte Kultur zeigte ein etwas anderes Verhalten als Mais und Rotklee. Im Mittel aller 5 Böden wurden aus KS Gö 100 \% P im Vergleich zur Ca-P-Variante, also die gleiche P-Menge von Gras aufgenommen. Aus KS Sh waren es sogar $119 \%$. Interessant ist, dass das Gras auf dem Quarzsand aus beiden KS im 3. Jahr deutlich mehr P aufnehmen konnte (KS Gö 55 \%, KS Sh $151 \%$ ) als aus $\mathrm{CaHPO}_{4}$. Auch auf dem P- armen Substrat Elliehausen bestand die Tendenz einer höheren P- Aufnahme des Grases im Vergleich zur Ca-P-Variante. Das Weidelgras war also offenbar in der Lage, den Phosphor im 3. Anbaujahr in gleicher Weise zu nutzen wie aus dem $\mathrm{CaHPO}_{4}$. Die Resultate der P- Entzüge des Weidelgrases, das erst nach einjähriger Inkubation in 8 Ackerböden angebaut worden war (Tabelle 12) verhielt sich ähnlich wie Mais bzw. Rotklee. Es hatte bei KS Gö- Düngung im Mittel der 8 Böden nur $85 \%$ des $\mathrm{P}$ der $\mathrm{CaHPO}_{4}-$ Variante aufgenommen. Eine Trennung zwischen den Schluffund Sandböden erbrachte zumindest bei der KS Gö- Anwendung keine klare Differenzierung zwischen den Böden. Die P- Entzüge lagen bei 84 bzw. 80 \% bei den Böden mit mäßiger P- Versorgung. Bei KS Sh- Anwendung erreichten die Pflanzen auf den 3 P- armen Schluff- und den drei Sandböden P- Entzüge von $109 \%$.

Aus diesen Resultaten leitet sich insgesamt der Schluss ab, dass die PAufnahme besonders aus dem KS Sh mit seinem engen Fe: $\mathbf{P}-$ Verhältnis $(1: 1,1)$, unabhängig von Bodenart und P-Gehalt der Böden, der aus 
$\mathrm{CaHPO}_{4}$ nahezu gleich war. Für Klärschlamm Gö traf das nicht zu. Unabhängig davon, ob die Pflanzen 1 Monat oder 13 Monate nach der PDüngung angebaut worden waren, lagen die P- Entzüge der Pflanzen (Mais, Rotklee, Weidelgras)nur bei ca. 76 bis $87 \%$ bezogen auf die $\mathrm{CaHPO}_{4}$ - Variante. Ausnahmen davon waren Mais auf den Böden RehburgLoccum und Düshorn (Brache) (Tabelle 8), Rotklee auf Boden Elliehausen und Weidelgras im 3. Anbaujahr, wo mit KS Gö der gleiche P- Entzug wie mit $\mathrm{CaHPO}_{4}$ erreicht wurde.

Eine wesentliche Ursache für die geringe P-Aufnahme, besonders auf den Schluffböden, ist auf die veränderte P-Dynamik in den Böden nach der Düngung mit den drei P-Quellen zurückzuführen. Durch eine $\mathrm{CaHPO}_{4}$-Düngung war auf allen Böden nach einem Monat die P-Sorption erwartungsgemäß gesunken. Aber nach der P-Applikation mit KS Gö war die P- Sorption z.B. auf den Böden Börry P-0 und P-80 auf 120 bzw. $160 \%$ der Kontrolle (P 0) signifikant angestiegen und blieb 6 Monate lang auf dieser Höhe (Tabelle 16). Bei den Sandböden stieg die P-Sorption nach 1 Monat nur maximal um $8 \%$ und war nach 6 Monaten gar nicht mehr nachweisbar (Tabelle 17). Dagegen trat auf den Sandböden infolge KS Sh- Anwendung kaum eine Anhebung der PSorption ein. Der Anstieg der P-Sorption auf den Schluffböden - insbesondere durch KS Gö - verursachte im Vergleich zur $\mathrm{CaHPO}_{4}$-Variante deutlich verminderte Konzentrationen an Orthophosphat $\left(\mathrm{P}_{\mathrm{i}}\right)$ in der Bodenlösung (Tabelle 14 und 15). Diese war in den meisten Fällen signifikant niedriger als in den KS Sh- Varianten. Die auf den Schluffböden gemessene geringere PAufnahme der Maispflanzen nach KS Gö- Düngung ist also offenbar auf die gesunkene P-Verfügbarkeit durch den eisenreichen KS Gö zurückzuführen. Interessanterweise fanden Zorn et al. (2001) auf einem Lößlehmboden, dass vier aufeinander folgende Kulturpflanzen nach Düngung mit Klärschlämmen, die bis $7 \% \mathrm{Fe}$ aus der P-Elimination enthielten, ebenfalls um bis zu $20 \%$ geringere P-Aufnahmen als Pflanzen nach $\mathrm{Ca}\left(\mathrm{H}_{2} \mathrm{PO}_{4}\right)_{2}$ - Düngung aufwiesen. Ferner konnten sie mit mehreren Methoden nachweisen, dass das Inlösunggehen von Phosphat in Böden, die mit solchen $\mathrm{Fe}$ - reichen Schlämmen gedüngt waren, deutlich geringer war als nach Zufuhr von Schlämmen mit P-Fällung durch Kalk oder mit biologischer P-Elimination. Auf diese als negativ zu wertenden Effekte 
von KS mit P-Elimination durch Eisensalze haben Fritsch und Werner (1989) bereits hingewiesen.

Während also auf den P- armen Schluffböden Börry P-0 und Elliehausen die Maispflanzen aus KS Gö weniger $\mathrm{P}$ als aus $\mathrm{CaHPO}_{4}$ aufnehmen konnten und auch weniger Trockenmasse bildeten (Abb. 5 und 6), bildeten die Pflanzen trotz ähnlich hoher P-Aufnahme aus KS Gö auf den Sandböden Rehburg und Düshorn teilweise sogar mehr Trockenmasse als Pflanzen mit $\mathrm{CaHPO}_{4}$ Düngung. Hier muss es neben der P-Versorgung noch andere Wirkungen des KS Gö geben, die für das Wachstum wichtig sind. Tabelle 19 zeigt eine mögliche Erklärung. Der KS Gö erhöhte auf Grund seines hohen Kalkgehaltes in allen Böden den $\mathrm{pH}-$ Wert. Dieser $\mathrm{pH}$ - Anstieg war für das Pflanzenwachstum aber nur auf den Sandböden bedeutsam, da deren pH-Werte in den Kontrollgefäßen bzw. den Gefäßen der $\mathrm{CaHPO}_{4}$ - und KS Sh- Varianten nicht im Optimum lagen. Bei pH-Werten $<5$ ist mit Al-Toxizität und erhöhter MnLöslichkeit zu rechnen (Scheffer/Schachtschabel, 2002). Das heißt, dass für das bessere Wachstum des Maises auf den Sandböden nach KS GöAnwendung neben der P-Zufuhr vor allem die reduzierte Bodenacidität verantwortlich gewesen sein dürfte.

Tabelle 19: $\mathrm{pH}$-Werte $\left(\mathrm{CaCL}_{2}\right)$ der Böden 1 Monat nach der Düngung mit $187 \mathrm{mg}$ P pro Gefäß (Mittelwerte aus 4 Wiederholungen)

\begin{tabular}{lcccc}
\hline Boden & Ohne P & $\mathrm{CaHPO}_{4}$ & KS Gö & KS Sh \\
\hline Börry P-80 & 6,2 & 6,2 & 7,3 & 6,2 \\
Börry P-0 & 7,2 & 7,3 & 7,6 & 7,2 \\
Elliehausen & 7,7 & 7,7 & 7,9 & 7,7 \\
Rehburg & 4,3 & 4,3 & 4,9 & 4,4 \\
Düshorn (Brache) & 4,9 & 4,9 & 5,6 & 4,9 \\
\hline
\end{tabular}

Ähnliches traf für den Rotklee zu (Tabelle 4), der auch auf Böden mit pHWerten $<5$ ebenfalls weniger gut wächst (Schilling, 2000, S. 165). Insgesamt gesehen ist also das vergleichsweise gute Wachstum des Maises und dessen 
relativ hohe P-Aufnahme auf den Sandböden Rehburg und Düshorn nach KS Gö- Applikation sowohl auf die Kalkwirkung als auch auf die P-Zufuhr zurückzuführen. Ein indirekter Beweis für diesen Schluss ergibt sich aus dem Experiment des Kapitels 2.3 und von den Ergebnissen des Weidelgrases nach der 1-jährigen Inkubationszeit. Dort gab es zusätzlich für drei Böden Varianten in denen Kalk zum Boden ohne P- Düngung zugegeben wurde. Die Auswirkung auf Wachstum und P- Entzug ging aus Tabelle 13 hervor. Man erkennt dort, dass auf dem Schluff- Boden von Dorstadt die Kalkung keinen positiven Effekt auf Wachstum und P- Aufnahme des Grases hatte. Dagegen bildeten die Pflanzen auf dem Sandböden Düshorn (Brache) und Rehburg- Loccum deutlich mehr Sprossmasse, ohne aber ihre P- Aufnahme zu erhöhen. Das ist ein klarer Hinweis, dass auf diesen Böden die $\mathrm{pH}$ - Anhebung durch die Kalkung die PDynamik der Sandböden kaum beeinflusst, aber andere negative Wachstumsfaktoren (erhöhte $\mathrm{Al}^{3+}$ - Konzentrationen bei $\mathrm{pH} 4$ 4) positiv für die Pflanzen verändert hat. Eine Nutzung von KS mit hohen Kalkgehalten ist für die Landwirtschaft dann interessant, wenn die Kosten für den KS sehr gering sind, da sonst Kalk zur Regulierung der Bodenreaktion eingesetzt werden müsste.

Ein besonderes Phänomen trat auf dem Boden Börry P-0 auf (Abbildung 6). Während der geringere Ertrag in der Variante KS Gö mit der geringen PAufnahme korrespondierte, traf das bei KS Sh nicht zu. Dort war der P-Entzug ebenso groß wie bei $\mathrm{CaHPO}_{4}$-Düngung, aber der Ertrag war signifikant um $19 \%$ niedriger. Eine mögliche Erklärung lässt sich aus der Kornbildung des Maises ablesen. Zur Ernte waren folgende Kornerträge bei den einzelnen Varianten gefunden worden: Kontrolle: 9 g; $\mathrm{CaHPO}_{4}: 25$ g; KS Gö: 17 g; KS Sh: $17 \mathrm{~g}$ (nicht in Tabellen dargestellt). Aus früheren Arbeiten (Römer und Schilling, 1986) ist bekannt, dass der P-Ernährungszustand der Jungpflanzen über die Anlage der generativen Organe entscheidet. Man erkennt, dass zwar mit der KS-Düngung (KS Gö und KS Sh) doppelt so viel Kornmasse gebildet wurde wie in der P-0-Variante, aber wesentlich weniger als bei $\mathrm{CaHPO}_{4}$-Ernährung. Das heißt, das Phosphat, welches man in den Pflanzen zur Ernte in der Variante KS Sh gefunden hat, ist vermutlich erst in späten Abschnitten des Wachstums aufgenommen worden und ist deshalb zur Anlage der generativen Organe zu 
spät gekommen. Wenn diese Zusammenhänge tatsächlich zutreffen, dann liegt die Vermutung nahe, dass ein zeitlich begrenzter hoher P-Bedarf in frühen Entwicklungsstadien mancher Kulturpflanzen mit diesem Klärschlamm (KS Sh) nicht gedeckt werden kann, da sein Phosphat erst mit der Zeit verfügbar wird.

Es bleibt die Frage nach der längerfristigen P-Düngewirkung der Klärschlämme. Was die P-Aufnahme der Rotkleepflanzen betrifft, so zeigen sie im Mittel für die 5 Ackerböden, bezogen auf die Ca-P-Variante, nahezu die gleichen relativen P-Entzüge wie der Mais (KS Gö 89 \%, KS Sh 107 \%, Tab. 5). Lässt man den extrem $P$ - armen und carbonathaltigen Unterboden (= Löß) aus Elliehausen aus der Betrachtung heraus, so liegt die P-Aufnahme bei KS Gö in Bezug auf die Ca-P-Variante nur bei $82 \%$, die des KS Sh aber bei $100 \%$. Das heißt, der nachgebaute Rotklee war im Vergleich zu Mais bis auf das Substrat Löß (Elliehausen) nicht in der Lage, das P im KS Gö im 2. Nutzungsjahr gleich oder besser zu nutzen als den Phosphor aus $\mathrm{CaHPO}_{4}$. Das überrascht, denn vom Rotklee ist bekannt, dass er bei P-Unterversorgung verstärkt organische Säureanionen (Citrat, Oxalacetat) exsudiert (Gerke, 1995). Für Mais trifft das viel weniger zu (Beißner, 1997). Solche Anionen erhöhen die P-Löslichkeit (Gerke, 1992; Keller, 2000) vermutlich im Wesentlichen über einen Ligandenaustausch des sorbierten Phosphates (Gerke, 1992). Offenbar ist aber das Phosphat im KS Gö wesentlich fester gebunden als im KS Sh bzw. als das Phosphat, das mit $\mathrm{CaHPO}_{4}$ (= Mineraldünger) in den Boden gelangt war.

Interessant ist, dass auch Weidelgras das $\mathrm{P}$ aus KS Gö nicht besser nutzen konnte als das $\mathrm{P}$ aus $\mathrm{CaHPO}_{4}$, selbst wenn beide Dünger 1 Jahr im Boden umgesetzt worden waren. Weidelgras hat eine 10 mal höhere Wurzellängendichte als manche dikotyle Pflanze (Egle, pers. Mitteilung). Das könnte bedeuten, dass mehr Wurzeln zu Düngerpartikeln Kontakt haben als z.B. die Wurzeln von Rotklee. Aber offenbar ist auch Weidelgras nicht in der Lage, das P aus KS Gö besser zu nutzen als das aus KS Sh, weil dieses fest an Eisenoxidhydrate gebunden ist.

Insgesamt gesehen gibt es in Bezug auf die längerfristige Nutzung des $\mathrm{P}$ nur geringe Unterschiede zwischen den zwei KS. Während der P- Entzug aus KS 
Sh auf den typischen Ackerböden (Börry, Rehburg, Düshorn) in allen drei Versuchsjahren dem aus $\mathrm{CaHPO}_{4}$ stets nahezu gleich war (100 bis104\%), lag der P- Entzug aus KS Gö in den ersten zwei Versuchsjahren auf den schwereren Böden nur bei $78-82 \%$ und stieg erst im 3. Jahr auf $87-95 \%$ an. Auf diesen Böden war also die Phosphorfreisetzung aus KS Gö verzögert, so dass insgesamt nur $90 \%$ der P- Ausnutzung in Vergleich zur $\mathrm{CaHPO}_{4-}$ Düngung erreicht wurden. Auf den Sandböden trat diese Verzögerung nicht ein.

\section{Zum Schluss soll noch einmal auf die Frage eingegangen werden, warum} die zwei Klärschlämme so verschiedene Eisengehalte besitzen und was dies für die praktische Anwendung bedeutet. Wenn ein Klärschlamm wie der KS Gö $65 \mathrm{~kg} \mathrm{Fe} / \mathrm{t}$ TM enthält und alle drei Jahre $5 \mathrm{t} \mathrm{TM} /$ ha ausgebracht werden dürfen, so gelangen mit einer solchen Applikation $325 \mathrm{~kg} \mathrm{Fe}$ in Form verschiedener $\mathrm{Fe}$ - Verbindungen zusätzlich zum $\mathrm{Fe}$, das schon ohne $\mathrm{Fe}-$ Zugabe im Klärschlamm ist, in den Boden. Das ist eine Menge, die in der Mehrzahl der Fälle mit hoher Sicherheit den Gehalt des Oberbodens an oxalatlöslichem Eisen erhöht (vgl. Gö1- Varianten in der Abbildung 7). Gesteigerte Schlammmengen führen zu einem weiteren linearen Anstieg der $\mathrm{Fe}_{\mathrm{ox}}-$ Gehalte sowie der P-Sorption. D. h., dass tatsächlich dem applizierten Fe eine Schlüsselrolle bei der P-Sorption zukommt. Auf diesen Zusammenhang haben Fritsch und Werner (1989) hingewiesen. Von Seiten der Abwassertechnologie wird diskutiert, dass bei der P-Elimination durch Eisensalze Eisenphosphate (etwa Strengit) gebildet werden (Scheffer, 2000). Offenbar fehlen aber bisher eindeutige Identifizierungen der PFällungsprodukte. Wird $\mathrm{FeSO}_{4}$ wie im Fall des $\mathrm{KS}$ Gö vor dem Zulauf in das Belebungsbecken zugegeben, so dürfte $\mathrm{Fe}^{2+}$ durch den starken Luftstrom rasch oxidiert werden und als $\mathrm{Fe}(\mathrm{OH})_{3}$ ausfallen, denn der $\mathrm{pH}$-Wert liegt dort bei ca. 7. Die braune Farbe im Belebungsbecken spricht für diesen Prozess. Vermutlich wird das Phosphat gleichzeitig an diese Hydroxide gebunden. Im Klärwerk Salzhausen wird Phosphat mit $\mathrm{FeCl}_{3}$ im Belebungsbecken eliminiert. Auch hier dürften Fe- Hydroxide ausfallen, die das Phosphat binden. Damit wäre in beiden Klärwerken die P-Eliminierung beendet. Im Klärwerk Göttingen werden dann aber noch zur Konditionierung des Schlammes $\mathrm{FeCl}_{3}$ und $\mathrm{Ca}(\mathrm{OH})_{2}$ zugegeben. Beides als Lösung bzw. als Suspension. Damit fällt 
weiteres Fe- Hydroxid aus. Im Filterkuchen hatte damit der KS Gö ein molares Fe : P-Verhältnis von 1:0,3. Daraus geht hervor, dass die Eisenhydroxide/ oxide vermutlich noch eine hohe $\mathrm{P}$ - Sorptionskapazität haben, was für einen Schlamm mit einem molaren Fe : P-Verhältnis von 1:1,2 wie im Fall KS Sh nicht zutrifft. Damit ist das Absinken der $\mathrm{P}_{\mathrm{i}}$ - Konzentration sowohl in den Böden, die in den Gefäßen mit KS Gö inkubiert waren, zu erklären. Die neu in die Böden eingebrachten Sorbenten haben viele freie Sorptionsplätze und sorbieren daher Orthophosphationen aus der Bodenlösung. Diese Absenkung der $\mathrm{P}_{\mathrm{i}}$ Konzentration war noch 12 Monate nach der KS-Applikation zu messen (Tabelle 15). D. h., mit einer kurzfristigen Wiederfreisetzung des spezifisch gebundenen Phosphates ist also nicht zu rechnen. Hier stellt sich die Frage, ob ein längeres Zwischenlagern der Schlämme vor der Ausbringung auf die Felder zu einer Umwandlung junger, schlecht geordneter Fe-Oxide (Ferrihydrit) in gealterte Fe-Oxide führt, die dann eine geringere $\mathrm{P}$ - Sorption hätten (Schwertmann, 1998). Allerdings müsste auch die Nutzbarkeit der Phosphate dieser Schlämme extra geprüft werden. Ein Hinweis, dass sich die P- Sorption tatsächlich nach längerer Lagerung verringert, geht aus Tabelle 18 hervor. Dort ist zu erkennen, dass sich die P- Sorption im Boden unabhängig von der Höhe der KS- Menge des KS Gö innerhalb von 12 Monaten um ca. 10\% auf den Schluffböden und ca. $10-20 \%$ bei den Sandböden verringert.

Mit $5 \mathrm{t} \mathrm{KS} \mathrm{Gö} \mathrm{werden} \mathrm{außerdem} \mathrm{fast} 1000 \mathrm{~kg} \mathrm{Ca} / \mathrm{ha}$ vorrangig als basisch wirksamer Kalk, ausgebracht. Hier muss offen bleiben, wie viel von dem ursprünglichen $\mathrm{Ca}(\mathrm{OH})_{2}$ noch als solches vorlag oder bereits neutralisierter Kalk $\left(\mathrm{CaCO}_{3}\right)$ war. Auch $\mathrm{CaCO}_{3}$-Partikel adsorbieren Phosphat (Parfitt, 1978). Mit dem KS Gö wird im Boden auch örtlich eine hohe Ca-lonenkonzentration bei hohem pH auftreten. Das fördert die P-Bindung im Boden (Schwertmann und Amann, 1998) vermutlich durch die Ca-Phosphatbildung und zusätzlich die P-Bindung an $\mathrm{CaCO}_{3}$-Partikel. Die bei KS Gö gemessenen höheren PSorptionswerte werden vermutlich durch die Adsorption an Fe- Hydroxide/ Oxide, eine $\mathrm{P}$ - Sorption an $\mathrm{CaCO}_{3}$ und die Ca-Phosphatfällung verursacht.

Kalkapplikationen zu den untersuchten Böden waren nicht erfolgt, so dass eine Gewichtung der Anteile der P-Sorptionsprozesse nicht möglich ist. Dass bei 
den Sandböden die Regressionsgeraden viel höhere Bestimmtheitsmaße aufweisen (Abbildung 9), kann vielleicht damit erklärt werden, dass in den Sandböden mit ihren pH-Werten von 4,3 bzw. 4,9 (Tabelle 19) der applizierte Kalk teilweise neutralisiert worden ist. Die pH-Werte waren auf Werte um 5 bis 5,6 angestiegen (Tabelle 19). Damit trat zumindest auf den Sandböden keine PSorption an $\mathrm{CaCO}_{3}$ auf. Im Wesentlichen waren vermutlich nur die $\mathrm{Fe}$ Verbindungen als $\mathrm{P}$ - Sorbenten wirksam.

Insgesamt ergibt sich, dass die Verringerung der $\mathrm{P}_{\mathrm{i}}$-Konzentration in vier Böden offenbar eine Folge der erhöhten P- Sorption der Böden nach KS GöApplikation ist. Diese Zusammenhänge gelten offenbar für Böden mit Gehalten an lactatlöslichem $\mathrm{P}$ von mehr als $69 \mathrm{mg} \mathrm{kg}^{-1}$ (Börry P-80, Dorstadt P-20, Düshorn P-22). Eine Ausnahme macht Hodenhagen. Bei diesem Boden und bei allen anderen (Börry P-0, Dorstadt P-0, Elliehausen, Rehburg, Düshorn-Brache) stiegen auch nach KS Gö- Applikation die $\mathrm{P}_{\mathrm{i}}$-Werte etwas an oder blieben auf dem Niveau der Kontrolle (P 0). Angesichts obiger Argumente überrascht dieser Befund zunächst. Er wird vermutlich damit erklärbar, dass die P-Bindung in den genannten Böden, die alle eine sehr geringe bis mäßige P-Verfügbarkeit (Lactatwerte) aufweisen (Tabelle 9), fester ist, als die im KS Gö. Das wäre aber zu überprüfen.

\section{Schlussfolgerungen}

- Mais, Rotklee und Weidelgras nacheinander angebaut oder Weidelgras als 1. Kultur können sowohl auf schluffigen Böden als auch Sandböden $P$ aus einem $\mathrm{KS}$ mit $\mathrm{P}$ - Elimination $\left(\mathrm{FeCl}_{3}\right.$ im Belebungsbecken) ohne Konditionierung und einem molaren Fe: P- Verhältnis von 1:1,16 bzw. 0,86:1 nahezu gleiche $\mathrm{P}$ - Mengen aufnehmen wie bei $\mathrm{CaHPO}_{4}$ - Düngung.

Die Ausbringung eines solchen Klärschlamm ist zu empfehlen.

- Nicht generell empfohlen werden kann der KS Gö mit seinem weiten molaren Fe: P-Verhältnis von 1:0,33 bzw. 3:1 das aus der P- Elimination im Belebungsbecken $\left(\mathrm{FeSO}_{4}\right)$ sowie der Konditionierung mit $\mathrm{FeCl}_{3}+$ $\mathrm{Ca}(\mathrm{OH})_{2}$ resultiert. Dieser KS erlaubt P- Entzüge auf Schluffböden von nur 
$75 \%$ bei Mais, von $89 \%$ bei Rotklee und $99 \%$ bei Weidelgras im 3. Jahr. Auf Sandböden werden bei Mais und Weidelgras ca. 100\%, bei Rotklee nur 85\% der P- Entzüge von CaHPO4 erreicht. Diese befriedigende Wirkung auf Sandböden beruht weniger auf der P- Zufuhr als auf der Kalk- Zufuhr, die die $\mathrm{pH}-$ Werte der Sandböden anhob und damit "Säureschäden" minderte.

Klärschlämme wie der KS Gö mit dem molaren Fe: P- Verhältnis von 1:0,3 und dem hohen Kalkanteil verursachen in Böden mit mittlerer und hoher PVersorgung eine Erhöhung der P-Sorptionskapazität und eine Senkung der Orthophosphatkonzentration der Bodenlösung. Die Änderung dieser Parameter ist Ausdruck einer verminderten P- Verfügbarkeit in den Böden. Letztere reduziert die P- Aufnahme der Pflanzen. Diese als grundsätzlich zu betrachtenden Vorgänge wurden nur nach Anwendung von $2 \mathrm{KS}$ mit hohen bzw. niedrigen Fe- Gehalt gefunden. Sie sollten an einem größeren Spektrum von eisenhaltigen KS überprüft werden. Sollten sich diese Resultate bestätigen, ist der Schluss zu ziehen, dass zumindest bei der Schlammkonditionierung keine Eisensalze eingesetzt werden sollten. 
Teil II

Einjährige Experimente zur P-Aufnahme von Kulturpflanzen und $\mathrm{P}$ Verfügbarkeit im Boden nach Applikation von 13 Klärschlämmen und verschiedener P- Dünger in einen humosen Sandboden

\subsection{Vorbemerkungen und Versuchsziel}

In den vorangegangenen Versuchsserien wurde die P- Düngewirkung von zwei eisenhaltigen Klärschlämmen auf 9 Böden bzw. Substraten längerfristig (2 bis 3 Jahre) geprüft. In den nun folgenden Experimenten sollte das Spektrum der Klärschlämme beträchtlich erweitert werden (von 2 auf 13). Die Absicht war, Klärschlämme mit einem möglichst weiten Fe: P-Verhältnis (Einsatz von Fe zur P- Elimination und Schlammkonditionierung) bis hin zu einem engen Verhältnis (KS ohne Fe- Zugabe) auf ihre P- Düngewirkung zu prüfen. Das Ziel war, eine begründete Entscheidung über den zu tolerierenden Eisengehalt der Klärschlämme zu treffen. Um eventuell eine Aussage über eine mögliche Auswirkung von frisch gefällten Eisenhydroxiden in den Klärschlämmen auf die P- Verfügbarkeit zu erhalten, wurden auch Gefäße mit frisch gefällten FeHydroxiden angesetzt. Im Spektrum der 13 Klärschlämme gab es auch einige mit hohem Kalkgehalt. Da damit eine Beeinflussung der Boden- pH-Werte und der P- Löslichkeit in den Böden zu erwarten war, sind auch Gefäße ohne P aber mit Kalk gedüngt worden, um Aussagen über eine mögliche Kalkwirkung der KS machen zu können. Außerdem gab es kombinierte Applikationen von FeHydroxiden plus Kalk. Alle Klärschlämme wurden gefriergetrocknet und als gemahlene Pulver eingesetzt. Auf Grund der großen Zahl der KS wurde nur ein einzelner Boden benutzt. Es war ein Sandboden (Tabelle 21), sehr ähnlich dem Boden Düshorn (Brache) wie er bereits im Teil I benutzt worden war (Tabelle 3). Es wurde Weidelgras als Kulturpflanze ausgewählt. Im Drahthaus wurde Weidelgras (3 Schnitte) in Mitscherlichgefäßen kultiviert. Die P- Düngewirkung der KS wurde in diesen Experimenten mit einem in der Praxis üblichen PDünger, dem Triplephosphat, also $\mathrm{Ca}\left(\mathrm{H}_{2} \mathrm{PO}_{4}\right)_{2}$, verglichen. Des Weiteren kam auch Hyperphosphat (gemahlenes Rohphosphat) zur Anwendung, da der pHWert des Sandbodens Düshorn 5,4 war. Neben dem Hauptziel des Experimentes, die P- Düngewirkung der KS zu messen (TM- Bildung, P- 
Entzug), sollte auch der Einfluss auf die P- Verfügbarkeit der KS bzw. der anderen Zusätze getestet werden. Deshalb sind 4 Wochen nach Düngerapplikation (direkt vor der Aussaat) die Böden auf folgende Eigenschaften geprüft worden:

$\mathrm{P}_{\mathrm{i} \text { - Konzentration in der Bodenlösung }}$

P-Sorptionsvermögen

Gehalt an DL - löslichem P

Gehalt an oxalatlöslichem Fe

$\mathrm{pH}-$ Wert $\left(\mathrm{CaCl}_{2}, 0,01 \mathrm{M}\right)$

Nach der Ernte wurden die Boden- $\mathrm{pH}-$ Werte wieder gemessen. $\mathrm{Zu}$ den Methoden siehe Kapitel 2.3.3

\subsubsection{Verwendete Klärschlämme}

Die 13 benutzten Klärschlämme stammen aus 12 niedersächsischen Klärwerken und einer nordrhein-westfälischen Kläranlage (Gütersloh). Alle Klärschlammproben wurden als frisches Material im Juli 2000 gewonnen. Für die Analysen und Gefäßversuche wurden Unterproben gefriergetrocknet. Die wichtigen Kenndaten der 13 benutzten Klärschlämme sind in Tabelle 20 wiedergegeben. Weitere Angaben siehe Tabelle $8.14 \mathrm{im}$ Anhang. Die Klärschlämme sind nach ihrem molaren Fe / P-Verhältnis geordnet worden.

Man erkennt, die absoluten P- Gehalte der Klärschlämme schwankten zwischen $8777 \mathrm{mg} \mathrm{kg}^{-1}$ (Dollbergen) und $38690 \mathrm{mg} \mathrm{kg}^{-1}$ (Uelzen). Große Differenzen gab es auch in den Eisengehalten. KS Schöppenstedt besaß nur $7031 \mathrm{mg} \mathrm{kg}^{-1}$ während Einbeck $79018 \mathrm{mg} \mathrm{kg}^{-1}$ enthielt. Die P- Düngewirkung wurde aber bei gleichen Werten der P- Applikation geprüft, so dass die absoluten P- und FeGehalte für das Versuchsziel weniger wichtig sind als die molaren $\mathrm{Fe}$ : $\mathrm{P}$ Verhältnisse des jeweiligen Schlammes. Dieses Verhältnis ist im Klärschlamm Dollbergen am weitesten. Bei $1 \mathrm{Mol} F e$ enthält der Schlamm nur 0,24 Mol Phosphat bzw. auf 1 Mol P entfallen 4,2 Mole Fe. Das engste Verhältnis hat der 
KS Schöppenstedt mit $1: 7,94$ bzw. 0,1:1. Aus den Werten der CaO- Gehalte und $\mathrm{pH}$-Werte geht hervor, dass die KS aus Einbeck, Uelzen, Salzhausen und Schöppenstedt nicht mit Kalk konditioniert waren.

Tabelle 20: Wichtige Kenndaten der gefriergetrockneten Klärschlämme (Daten der LUFA Hameln)

\begin{tabular}{|c|c|c|c|c|c|c|}
\hline $\begin{array}{l}\mathrm{KS} \\
\mathrm{Nr}:\end{array}$ & Ort & $\begin{array}{c}P \\
\mathrm{mg} \mathrm{kg}^{-1}\end{array}$ & $\begin{array}{c}\mathrm{Fe} \\
\mathrm{mg} \mathrm{kg}^{-1}\end{array}$ & $\begin{array}{l}\text { Molar. Fe:P } \\
\text { Verhältnis }\end{array}$ & $\begin{array}{c}\mathrm{CaO} \\
\mathrm{mg} \mathrm{kg}^{-1}\end{array}$ & $\begin{array}{c}\mathrm{pH} \\
\mathrm{CaCl}_{2}\end{array}$ \\
\hline 1 & Dollbergen & 8777 & 65045 & $1: 0,24(4,2)$ & 235000 & 10,8 \\
\hline 2 & Göttingen & 11354 & 61810 & $1: 0,33(3,0)$ & 271000 & 11,2 \\
\hline 3 & Gronau & 12096 & 53118 & $1: 0,40(2,5)$ & 320000 & 12,0 \\
\hline 4 & Hameln & 19650 & 55783 & $1: 0,63(1,6)$ & 249000 & 10,1 \\
\hline 5 & Einbeck & 34236 & 79018 & $1: 0,77(1,3)$ & 58600 & 7,5 \\
\hline 6 & Herzberg & 24410 & 54325 & $1: 0,80(1,3)$ & 222000 & 7,95 \\
\hline 7 & Gütersloh & 13799 & 27493 & $1: 0,89(1,1)$ & 338000 & 12,1 \\
\hline 8 & Northeim & 13974 & 26434 & $1: 0,94(1,1)$ & 314000 & 10,3 \\
\hline 9 & Uelzen & 38690 & 54610 & $1: 1,26(0,8)$ & 64700 & 7,4 \\
\hline 10 & Osterode & 30087 & 42050 & $1: 1,27(0,8)$ & 218000 & 12,0 \\
\hline 11 & Salzhausen & 28602 & 37728 & $1: 1,35(0,7)$ & 35300 & 7,0 \\
\hline 12 & Uetze & 15197 & 10704 & $1: 2,51(0,4)$ & 390000 & 12,3 \\
\hline 13 & Schöppenstedt & 31485 & 7031 & $1: 7,94(0,1)$ & 48200 & 7,4 \\
\hline
\end{tabular}

\subsubsection{Verwendeter Boden}

Die Kenndaten des eingesetzten Bodens gibt Tabelle 21 wieder. Das Bodenmaterial wurde aus der Ackerkrume $(0-30 \mathrm{~cm})$ entnommen. Der Boden Düshorn (Kreis Soltau-Fallingbostel) ist ein anlehmiger Sandboden pleistozänen Ursprungs mit niedrigem Lactat-P- Gehalt, der sich an der Grenze zwischen Gehaltsklasse B und C befindet. 
Tabelle 21: Kenngrößen des Versuchsbodens

\begin{tabular}{cccccccc}
\hline Ort & $\begin{array}{c}\text { Ton } \\
\text { Schluff }\end{array}$ & $\begin{array}{c}\text { Sand } \\
\%\end{array}$ & $\begin{array}{c}\mathrm{pH} \\
\%\end{array}$ & $\begin{array}{c}\text { Humus } \\
\mathrm{CaCl}_{2}\end{array}$ & $\begin{array}{c}\text { DL-Extr. } \\
\%\end{array}$ & $\begin{array}{c}\mathrm{Fe}_{\text {ox. }} \\
\mathrm{mg} \mathrm{P} \mathrm{kg}^{-1}\end{array}$ & $\mathrm{mg} \mathrm{Fe} \mathrm{kg}^{-1}$ \\
\hline $\begin{array}{c}\text { Düshorn } \\
\text { (Brache) }\end{array}$ & 1 & 6 & 93 & 5,4 & 3,8 & 45 & 1445 \\
\hline
\end{tabular}

\subsubsection{Genereller Versuchsaufbau}

Tabelle 22 gibt die Varianten des geplanten Gefäßversuches wieder. Dem Ziel entsprechend gab es eine Kontrolle (K0) ohne P- Düngung sowie die 13 Varianten mit den gefriergetrockneten Klärschlämmen (KS 1 bis KS 13). Verglichen werden sollten die KS- Varianten mit den $P$ - Düngern Hyperphosphat (Hy) bzw. Triplesuperphosphat (TSP). Es wurde nur mit einer PMenge pro Gefäß gearbeitet. Da sich die P-Applikation von $187 \mathrm{mg} \mathrm{P} \mathrm{je}$ Mitscherlichgefäß in den Experimenten mit den zwei Klärschlämmen KS Gö und KS Sh bewährt hatte (vgl. dortige Resultate), wurde hier mit der gleichen PMenge, also mit ca. $30 \mathrm{mg} \mathrm{P} \mathrm{kg}{ }^{-1}$ Boden (187 mg / $6 \mathrm{~kg}$ Boden je Mitscherlichgefäß), gedüngt. Die entsprechenden KS- Mengen $\mathrm{kg}^{-1}$ Boden finden sich in Tabelle $8.15 \mathrm{im}$ Anhang. Da mit den KS auch gefälltes Eisenhydroxid und Kalk in die Böden eingebracht wurde und deren Wirkung auf die P- Verfügbarkeit unklar war, wurden in die Gefäße mehrerer Varianten gestaffelte Mengen an Fe (gefälltes Fe- Hydroxid) oder / und $\mathrm{CaO}$ eingebracht. Die angegebenen Eisenmengen wurden aus definierten $\mathrm{FeCl}_{3}$ - Lösungen gewonnen, die mit $\mathrm{NaOH}$ versetzt wurden. Das gefällte $\mathrm{Fe}(\mathrm{OH})_{3}$ wurde abfiltriert, luftgetrocknet, mit wenig Boden zerrieben und unter die Böden der Gefäße gemischt. $\mathrm{CaO}$ wurde als p.A. Chemikalie eingesetzt. 
Tabelle 22: Versuchsvarianten der Gefäßversuche zur Prüfung von 13 Klärschlämmen auf ihre P-Düngewirksamkeit (P-Applikation: $30 \mathrm{mg}$ $P \mathrm{~kg}^{-1}$ Boden)

P- Varianten P $0 \quad$ Kontrolle, Versuchsboden ohne P

Fe $1 \quad$ Gefälltes Eisenhydroxid, $735 \mathrm{mg} \mathrm{Fe} \mathrm{kg}^{-1}$ Boden, ohne P

Fe 2 Gefälltes Eisenhydroxid, $235 \mathrm{mg} \mathrm{Fe} \mathrm{kg}^{-1}$ Boden, ohne P

Ca $1 \quad K a l k$ als $\mathrm{CaO}, 484 \mathrm{mg} \mathrm{CaO} \mathrm{kg}^{-1}$ Boden, ohne $\mathrm{P}$

Ca 2 Kalk als $\mathrm{CaO}, 161 \mathrm{mg} \mathrm{CaO} \mathrm{kg}^{-1}$ Boden, ohne $\mathrm{P}$

Fe 1 / Ca 1 Gefälltes Eisenhydroxid, $735 \mathrm{mg} \mathrm{Fe}$ plus $484 \mathrm{mg} \mathrm{CaO} \mathrm{kg}^{-1}$ Boden, ohne $\mathrm{P}$

Hy Hyperphosphat $13,5 \% \mathrm{P}$

TSP Triplesuperphosphat $20 \% \mathrm{P}$

KS 1 Klärschlamm Dollbergen

KS 2 Klärschlamm Göttingen

KS 3 Klärschlamm Gronau

KS $4 \quad$ Klärschlamm Hameln

KS $5 \quad$ Klärschlamm Einbeck

KS $6 \quad$ Klärschlamm Herzberg

KS $7 \quad$ Klärschlamm Gütersloh

KS 8 Klärschlamm Northeim

KS 9 Klärschlamm Uelzen

KS 10 Klärschlamm Osterode

KS 11 Klärschlamm Salzhausen

KS 12 Klärschlamm Uetze

KS 13 Klärschlamm Schöppenstedt

\subsubsection{Durchführung des Gefäßversuches mit Weidelgras unter Freilandbedingungen (Drahthaus)}

In diesem Experiment wurden Mitscherlich-Gefäße benutzt. Die P- Gabe betrug $30 \mathrm{mg} \mathrm{kg}^{-1}$ Boden (187 mg $P$ je Gefäß bzw. je 6 kg Boden). Am 18.01.2001 
wurde der Versuch angesetzt und die folgenden Nährstoffmengen je Gefäß eingearbeitet:

187 mg P als Klärschlamm bzw. als Triplesuper- oder Hyperphosphat

$1,0 \mathrm{~g} \mathrm{~N} \quad$ als Calciumnitrat $\mathrm{Ca}\left(\mathrm{NO}_{3}\right)_{2} \cdot 4 \mathrm{H}_{2} \mathrm{O}$

$1,0 \mathrm{~g} \mathrm{~K} \quad$ als Kaliumsulfat $\mathrm{K}_{2} \mathrm{SO}_{4}$

$0,2 \mathrm{~g} \mathrm{Mg} \quad$ als Magnesiumsulfat $\mathrm{MgSO}_{4} \cdot 7 \mathrm{H}_{2} \mathrm{O}$

Die Gefäße wurden im feuchten Zustand (ca. $50 \%$ der maximalen Wasserkapazität) 30 Tage ruhen gelassen. Dann wurden vor der Aussaat Bodenproben genommen, um die Orthophosphatkonzentrationen der Bodenlösungen, das P-Sorptionsvermögen, das DL- Phosphat, die pH-Werte und die $\mathrm{Fe}_{\mathrm{ox}^{-}}$Gehalte $\mathrm{zu}$ analysieren. Am 29.03.2001 wurden die Weidelgrassamen, Sorte LIRASAND (3g / Gefäß, entspricht ca. 100 Samen) ausgesät. Die Pflanzen wurden im Drahthaus kultiviert. Gegossen wurde auf ca. 65\% der maximalen Wasserkapazität. Es wurden 3 Schnitte geerntet. Nach dem ersten und zweiten Schnitt wurden jeweils $0,6 \mathrm{~g} N$ in Form von Ammoniumnitrat nachgedüngt. Das Weidelgras wurde am 29.05, am 30.06 und am 04.08.2001 geschnitten. Die Trockenmassen der Schnitte wurden ermittelt und das Erntegut zur P- Analyse aufbereitet.

\subsubsection{Ergebnisse}

\subsubsection{Erträge und P- Entzüge des Grases}

Tabelle 23 gibt die Erträge und P- Entzüge nur der Kontrollgefäße (ohne $P$ ) und der Gefäße mit den zwei P- Düngern TSP und Hyperphosphat wieder, um zu sehen, ob auf dem Boden $P$ ertragsbegrenzend und damit die Versuchsanstellung zur Prüfung der P- Düngewirkung der 13 KS geeignet ist.

Man erkennt, dass bereits mit Hyperphosphat signifikant 10\% Mehrertrag erzielt und $11 \%$ mehr $\mathrm{P}$ aufgenommen wurden als in der Kontrollvariante. Mit Triplephosphat betrug die Ertragssteigerung sogar 39\% und die Erhöhung der 
P- Aufnahme 49\%. Bei allen 3 Grasschnitten gab es signifikant erhöhte PAufnahmen.

Damit ist die Versuchsanstellung gut geeignet, die P- Düngewirkung der KS im Vergleich zum Triplephosphat zu prüfen.

Tabelle 23: Trockenmasseertrag und P-Entzug des Weidelgrases in Abhängigkeit von der mineralischen P-Quelle (1. bis 3. Schnitt) sowie die Relativwerte bezogen auf die Kontrolle $(P 0)$ ohne $P$ Düngung

\begin{tabular}{|c|c|c|c|c|c|c|c|c|}
\hline \multirow[t]{3}{*}{ Variante } & \multicolumn{4}{|c|}{ TM-Ertrag g/Gefäß } & \multicolumn{4}{|c|}{ Relativ \% } \\
\hline & \multicolumn{3}{|c|}{ Ernte } & \multirow[b]{2}{*}{ Gesamt } & \multicolumn{3}{|c|}{ Ernte } & \multirow[b]{2}{*}{ Gesamt } \\
\hline & 1. & 2. & 3. & & 1. & 2. & 3. & \\
\hline Kontrolle & 9,11 & 20,14 & 17,83 & 47,09 & 100 & 100 & 100 & 100 \\
\hline Hyper-P & 9,77 & 23,36 & 18,92 & 52,04 & 107 & 113 & 106 & 110 \\
\hline Triple-P & 26,03 & 23,77 & 16,30 & 66,10 & 286 & 115 & 91 & 139 \\
\hline \multicolumn{9}{|l|}{$\mathrm{GD}_{5 \%}$} \\
\hline \multirow[t]{2}{*}{ Tukey } & & & & 4,25 & & & & 9 \\
\hline & \multicolumn{4}{|c|}{ P-Entzug mg/Gefäß } & \multicolumn{4}{|c|}{ Relativ \% } \\
\hline Kontrolle & 16,2 & 41,3 & 35,0 & 92,6 & 100 & 100 & 100 & 100 \\
\hline Hyper-P & 17,2 & 46,1 & 39,8 & 103,1 & 106 & 112 & 114 & 111 \\
\hline Triple-P & 48,8 & 47,1 & 42,4 & 138,4 & 301 & 114 & 121 & 149 \\
\hline \multicolumn{9}{|l|}{$\mathrm{GD}_{5 \%}$} \\
\hline Tukey & & & & 9,92 & & & & 11 \\
\hline
\end{tabular}

Die 13 KS hatten folgende Wirkung auf die Graserträge (Tabelle 24): Bezogen auf die Kontrollvariante ( $P$ 0) wurden im Mittel aller KS $23 \%$ mehr Sprossmasse gebildet. Mit KS 1 und 2 gab es keine signifikanten Ertragssteigerungen, alle anderen KS bewirkten signifikante Ertragserhöhungen. Auf Basis der statistischen Analyse (GD 5\%) und ausgehend von der TSP- Variante ergaben sich folgende 2 Gruppen: 
(1) 0 - 15\% Mehrertrag: KS 1 und 2 (nicht signifikant verschieden von P 0)

(2) $>15 \%$ Mehrertrag: KS 3 bis 13

Die Gruppe aus KS 3, 6, 8, 9, 11, 12 und 13 war nicht signifikant verschieden von der Triplesuperphosphatvariante. Mit den betreffenden KS wurde also statistisch gesehen die gleiche Biomasse wie mit Triplesuperphosphat produziert.

Die P- Entzüge gehen aus Tabelle 25 hervor. Aus allen mit KS gedüngten Gefäßen, außer KS1, wurde signifikant mehr P aufgenommen im Vergleich zur Kontrolle. Die insgesamt 7\% mehr P- Entzug der Variante KS 1 resultierten vorzugsweise aus dem P- Entzug des 1. Schnittes (+ 108\%). Aber mit dem 3. Schnitt wurden nur 56 \% im Vergleich zu P 0 aufgenommen.

In Bezug auf die P- Entzüge ergab sich für die $\mathrm{KS}$ entsprechend der Grenzdifferenz von $11 \%$ und bei Bezug auf die TSP- Variante folgende Gruppierung:

(1) 0 - 11\% mehr P- Entzug: KS 1 (nicht signifikant verschieden von P 0)

(2) $>11 \%$ mehr P- Entzug erreichten die KS 2 bis 13

Die Gruppe aus KS 6, 8, 9, 10, 11, 12 und 13 unterschied sich in Bezug auf den P- Entzug des Grases nicht signifikant von dem aus Triplesuperphosphat, d.h. ihr Phosphat war für die Pflanzen gleich verfügbar wie aus TSP.

Man erkennt, dass mit den KS 6, 8, 9, 11, 12 und 13 sowohl die höchsten PEntzüge als auch die größten Mehrerträge erzielt wurden. Ihre Werte waren von denen des Triplesuperphosphat statistisch nicht verschieden. Die völlige Gleichheit im P- Entzug wurde nur mit den KS 12 und 13 erreicht (Tabelle 25).

Setzt man die P- Menge, die das Gras aus dem Triplesuperphosphat aufgenommen hat (138 mg - $93 \mathrm{mg}$ aus der Kontrollvariante $=45 \mathrm{mg}$ ) gleich $100 \%$, so ergeben sich die Relativwerte der P- Entzüge für die $13 \mathrm{KS}$ in Tabelle 26. 
Tabelle 24: Trockenmasseerträge des Weidelgrases in Abhängigkeit von der PQuelle (1. bis 3. Schnitt) sowie die Relativerträge bezogen auf die Kontrolle $(P$ O) ohne P-Düngung

\begin{tabular}{|c|c|c|c|c|c|c|c|c|}
\hline \multirow[t]{3}{*}{ Variante } & \multicolumn{4}{|c|}{ TM-Ertrag g/Gefäß } & \multicolumn{4}{|c|}{ Relativ \% } \\
\hline & \multicolumn{3}{|c|}{ Ernte } & \multicolumn{5}{|c|}{ Ernte } \\
\hline & 1. & 2. & 3. & Gesamt & 1. & 2. & 3. & Gesamt \\
\hline P 0 & 9,1 & 20,1 & 17,8 & 47,1 & 100 & 100 & 100 & 100 \\
\hline TSP & 26,0 & 23,8 & 16,3 & 66,1 & 286 & 115 & 91 & 139 \\
\hline KS 1 & 19,0 & 20,9 & 6,8 & 46,7 & 209 & 101 & 38 & 98 \\
\hline KS 2 & 21,3 & 21,2 & 10,2 & 52,7 & 233 & 103 & 57 & 111 \\
\hline KS 3 & 25,2 & 23,7 & 11,5 & 60,4 & 276 & 115 & 65 & 127 \\
\hline KS 4 & 22,4 & 24,0 & 8,9 & 55,3 & 246 & 116 & 50 & 116 \\
\hline KS 5 & 19,7 & 25,2 & 11,4 & 56,3 & 216 & 122 & 64 & 118 \\
\hline KS 6 & 25,0 & 26,1 & 11.0 & 62,1 & 274 & 126 & 62 & 130 \\
\hline KS 7 & 23,0 & 21,6 & 11,5 & 56,0 & 252 & 104 & 64 & 118 \\
\hline KS 8 & 24,3 & 25.0 & 10,1 & 59,3 & 266 & 121 & 57 & 125 \\
\hline KS 9 & 20,2 & 26,3 & 14,1 & 60,5 & 221 & 127 & 79 & 127 \\
\hline KS 10 & 22,4 & 23,5 & 10,9 & 56,8 & 246 & 114 & 61 & 119 \\
\hline KS 11 & 27,4 & 24,7 & 12,1 & 64,1 & 300 & 120 & 68 & 135 \\
\hline KS 12 & 28,5 & 26,8 & 12,8 & 68,0 & 313 & 130 & 72 & 143 \\
\hline KS 13 & 25,9 & 22,9 & 12,1 & 61.0 & 284 & 111 & 68 & 128 \\
\hline KS $\varnothing$ & 23,4 & 24.0 & 11,0 & 58,4 & 257 & 116 & 62 & 123 \\
\hline $\mathrm{GD}_{5 \%}$ Tukes & & & & 7,16 & & & & 15 \\
\hline
\end{tabular}


Tabelle 25: P-Entzüge des Weidelgrases in Abhängigkeit von den P-Quellen (1. bis 3. Schnitt) sowie die Relativwerte bezogen auf die Kontrolle (P 0) ohne P-Düngung

\begin{tabular}{|c|c|c|c|c|c|c|c|c|}
\hline \multirow[t]{3}{*}{ Variante } & \multicolumn{4}{|c|}{ P-Entzug mg/Gefäß } & \multicolumn{4}{|c|}{ Relativ \% } \\
\hline & \multicolumn{3}{|c|}{ Ernte } & \multicolumn{5}{|c|}{ Ernte } \\
\hline & 1. & 2. & 3. & Gesamt & 1. & 2. & 3. & Gesamt \\
\hline P0 & 16,2 & 41,3 & 35,0 & 92,6 & 100 & 100 & 100 & 100 \\
\hline TSP & 48,8 & 47,1 & 42,4 & 138,4 & 301 & 114 & 121 & 149 \\
\hline KS 1 & 33,8 & 45,4 & 19,5 & 98,6 & 208 & 110 & 56 & 107 \\
\hline KS 2 & 44,3 & 45,5 & 28,9 & 118,7 & 273 & 110 & 83 & 128 \\
\hline KS 3 & 40,6 & 49,7 & 33,7 & 124,0 & 250 & 120 & 96 & 134 \\
\hline KS 4 & 45,3 & 50,3 & 26,1 & 121,6 & 279 & 122 & 74 & 131 \\
\hline KS 5 & 40,0 & 52,3 & 33,7 & 126,0 & 247 & 127 & 96 & 136 \\
\hline KS 6 & 48,0 & 53,3 & 32,2 & 133,4 & 296 & 129 & 92 & 144 \\
\hline KS 7 & 48,6 & 45,3 & 31,1 & 125,0 & 300 & 110 & 89 & 135 \\
\hline KS 8 & 45,2 & 52,9 & 29,7 & 127,8 & 278 & 158 & 85 & 138 \\
\hline KS 9 & 36,8 & 55,3 & 38,1 & 130,2 & 227 & 134 & 109 & 141 \\
\hline KS 10 & 42,9 & 54,1 & 34,0 & 130,9 & 264 & 131 & 97 & 141 \\
\hline KS 11 & 44,8 & 54,0 & 36,5 & 135,3 & 276 & 131 & 104 & 146 \\
\hline KS 12 & 55,4 & 48,3 & 34,8 & 138,4 & 341 & 117 & 99 & 149 \\
\hline KS 13 & 53,8 & 48,2 & 35,2 & 137,2 & 332 & 117 & 100 & 148 \\
\hline KS $\varnothing$ & 44,0 & 50,3 & 31,6 & 126,0 & 271 & 122 & 90 & 136 \\
\hline $\mathrm{GD}_{5 \%}$ Tukey & & & & 9,77 & & & & 11 \\
\hline
\end{tabular}


Tabelle 26: Relative P-Entzüge aus den 13 Klärschlämmen bezogen auf die $P$ Entzüge aus TSP (3 Gruppen) (P-Entzug aus Triplesuperphosphat $=100 \%)$

\begin{tabular}{lllc}
\hline Variante & $\begin{array}{l}\text { Gruppe 1 } \\
0-62 \%\end{array}$ & $\begin{array}{l}\text { Gruppe 2 } \\
63-81 \%\end{array}$ & $\begin{array}{c}\text { Gruppe 3 } \\
82-100 \%\end{array}$ \\
\hline KS 1 & 13 & & \\
KS 2 & 57 & 69 & \\
KS 3 & & 63 & \\
KS 4 & & \\
KS 5 & & & \\
KS 6 & & 71 & \\
KS 7 & 77 & 89 \\
KS 8 & & & 84 \\
KS 9 & & 93 \\
KS 10 & & & 100 \\
KS 11 & & 97 \\
KS 12 & & & 100 \\
KS 13 & & & \\
TSP & & & \\
\hline
\end{tabular}

$\mathrm{GD}_{5 \%}($ Tukey $)=18 \%$

\section{Man erkennt:}

82 - 100\% der P-Aufnahme aus TSP (Gruppe 3) erreichten die KS 6, 9, 10, 11, 12,13 . Ihre Werte des P- Entzuges waren statistisch von dem aus TSP nicht zu trennen. Also waren sie statistisch gesehen dem TSP gleichwertig.

Weniger als $82 \%$ der P- Aufnahme aus TSP (Gruppe 2) erreichten die KS 1, 2 , 3, 4, 5, 7 und 8. Ihre Werte lagen signifikant unter dem aus TSP und weniger als $62 \%$ der P- Aufnahme aus TSP (Gruppe 1)erreichten der KS 1 und KS 2. Ihre Werte lagen signifikant unter den P- Aufnahmewerten der Gruppe 3. Ihre $\mathrm{P}$ - Verwertung war am geringsten. 
Tabelle 27 zeigt den Einfluss gestaffelter Fe- Hydroxid-, Ca- Hydroxid - bzw. Fe I Ca- Hydroxidmengen im Versuchsboden ohne P- Düngung.

Tabelle 27: TM-Ertrag und P-Entzug von Weidelgras nach Applikation von $\mathrm{Fe}(\mathrm{OH})_{3}$ (Variante $\mathrm{Fe}$ 1, $\mathrm{Fe} 2$ ), $\mathrm{Ca}(\mathrm{OH})_{2}$ (Ca 1, Ca 2) bzw. $\mathrm{Fe}(\mathrm{OH})_{3} / \mathrm{Ca}(\mathrm{OH})_{2}$ (Variante $\mathrm{Fe} / \mathrm{Ca}$ ); Kontrolle $(\mathrm{P} \mathrm{O})=100 \%$

\begin{tabular}{|c|c|c|c|c|c|c|c|c|}
\hline \multirow[t]{3}{*}{ Variante } & \multicolumn{4}{|c|}{ TM-Ertrag g/Gefäß } & \multicolumn{4}{|c|}{ Relativ \% } \\
\hline & \multicolumn{3}{|c|}{ Ernte } & \multicolumn{5}{|c|}{ Ernte } \\
\hline & 1. & 2. & 3. & Gesamt & 1. & 2. & 3. & Gesamt \\
\hline Kontrolle & 9,1 & 20,6 & 17,8 & 47,6 & 100 & 100 & 100 & 100 \\
\hline Fe 1 & 11,8 & 23,3 & 17,9 & 53,0 & 130 & 113 & 100 & 111 \\
\hline Fe 2 & 12,3 & 23,8 & 16,2 & 52,3 & 135 & 115 & 91 & 110 \\
\hline Ca 1 & 16,9 & 26,0 & 15,6 & 58,5 & 186 & 126 & 88 & 123 \\
\hline Ca 2 & 16,0 & 27,0 & 14,6 & 57,7 & 176 & 131 & 82 & 121 \\
\hline $\mathrm{Fe} / \mathrm{Ca}$ & 16,5 & 23,0 & 15,0 & 54,5 & 181 & 111 & 84 & 115 \\
\hline \multirow[t]{2}{*}{$\mathrm{GD}_{5 \%}$ Tuk. } & & & & 4,83 & & & & 10 \\
\hline & \multicolumn{4}{|c|}{ P-Entzug mg/Gefäß } & \multicolumn{4}{|c|}{ Relativ \% } \\
\hline Kontrolle & 16,2 & 41,3 & 35,0 & 92,6 & 100 & 100 & 100 & 100 \\
\hline Fe 1 & 19,4 & 47,6 & 39,8 & 106,8 & 120 & 115 & 114 & 115 \\
\hline $\mathrm{Fe} 2$ & 21,0 & 46,3 & 38,0 & 105,4 & 130 & 112 & 109 & 114 \\
\hline Ca 1 & 26,51 & 48,2 & 38,8 & 113,5 & 163 & 117 & 111 & 123 \\
\hline Ca 2 & 26,3 & 51,0 & 35,6 & 112,8 & 162 & 123 & 102 & 122 \\
\hline $\mathrm{Fe} / \mathrm{Ca}$ & 27,7 & 44,7 & 39,0 & 111,4 & 171 & 108 & 111 & 120 \\
\hline $\mathrm{GD}_{5 \%}$ Tuk. & & & & 9,67 & & & & 10 \\
\hline
\end{tabular}

Was die Trockenmasseerträge betrifft, so wurden sie durch die FeHydroxidzugaben im 1. Schnitt um ca. 30\%, im 2. Schnitt um 14\% und im 3. Schnitt nicht erhöht, so dass im Mittel die TM- Erträge um ca. 10\% anstiegen. Dieser Wert liegt auf der Sicherungsgrenze, ist also wenig sicher. Die $\mathrm{Ca}(\mathrm{OH})_{2}$ - Zufuhr erhöhte die Erträge des 1. und 2. Schnittes deutlich, senkte aber die des 3. Schnittes. Insgesamt stieg der TM- Ertrag aber signifikant um ca. 22\%. 
Prinzipiell gleich verhielt sich das Gemisch Eisen- / Calciumhydroxid. Nach inm stieg der TM- Ertrag im Mittel um 15\%.

Interessant ist, dass nicht wie erwartet die P- Entzüge durch eine $\mathrm{Fe}(\mathrm{OH})_{3}-$ Zufuhr abfielen, sondern sogar etwas anstiegen. Es waren im Mittel der 3 Schnitte $14-15 \%$. War $\mathrm{Ca}(\mathrm{OH})_{2}$ gedüngt worden, stiegen die P- Entzüge um signifikant 20 bis $23 \%$ an.

Damit ergab sich insgesamt, dass die $\mathrm{Ca}(\mathrm{OH})_{2^{-}}$Zugabe Wachstum und PAufnahme eindeutig positiv beeinflusste, die $\mathrm{Fe}(\mathrm{OH})_{3^{-}}$Zugabe aber keinen gesicherten Einfluss auf diese Parameter ausübte.

\subsubsection{Bodenkenngrößen}

Die Tabellen 28 und 29 geben die Werte der Bodenkenngrößen wieder, die 30 Tage nach der Düngerapplikation in die Böden bestimmt wurden.

Die DL- Werte (Tabelle 28) wurden durch die Mineraldüngeranwendung (30 mg $\mathrm{P} / \mathrm{kg}$ ) von 38 auf $54 \mathrm{mg} \mathrm{P} / \mathrm{kg}$ Boden, also um $42 \%$, angehoben. Die Düngung mit den $13 \mathrm{KS}$ bewirkte ebenfalls Anstiege um 40 bis $66 \%$. Damit bewirkte kein KS einen geringeren Anstieg der DL- Werte als die Mineraldünger. Die DL- Werte von KS 3, 7, 8 und 12 erreichten sogar Werte von über $160 \%$ bezogen auf die Kontrolle ohne P. Eine Kalkapplikation (Varianten Ca 1, Ca 2, Fe / Ca) ohne P- Zufuhr ließ die DL- Werte um 8-13\% ansteigen.

Die Orthophosphatkonzentration ( $\mathrm{P}_{\mathrm{i}}$ - Konz.) in den Bodenlösungen verhielten sich dagegen anders (Tabelle 28). Triplephosphat erhöhte die $\mathrm{P}_{\mathrm{i}}$ - Werte signifikant auf $211 \%$, während Hyperphosphat keinen Effekt hatte. Die KS hatten sehr differenzierte Wirkungen. Die KS 1 und 2 senkten die $\mathrm{P}_{\mathrm{i}}$ - Werte stark ab (32 bzw. $61 \%$ ), während die KS 9, 11 und 13 die $P_{\mathrm{i}}$ - Werte auf über $120 \%$ anhoben. Die Werte der anderen Behandlungen lagen zwischen diesen zwei Gruppen. Die Applikation von Fe- Hydroxid bzw. Ca- Hydroxid senkte die $\mathrm{P}_{\mathrm{i}}$ - Werte $\mathrm{ab}$, wobei die Kalkapplikation deutlichere Veränderungen bewirkte und die Kombination Eisen/Calcium- Hydroxid die Pi- Werte besonders stark reduzierte. 
Die pH-Werte (Tabelle 28) wurden durch die zwei Mineraldünger nicht beeinflusst. Durch die KS gab es Anstiege um bis zu $20 \%$, d.h. einen Anstieg um ca. eine ganze Einheit von 5,4 auf 6,5. Während die Applikation von FeHydroxid keine $\mathrm{pH}$ - Änderung bewirkte, stiegen nach Kalkapplikation die $\mathrm{pH}$ Werte wie erwartet an.

Tabelle 28: Einfluss der P-Mineral- und Klärschlammdüngung (KS) auf den

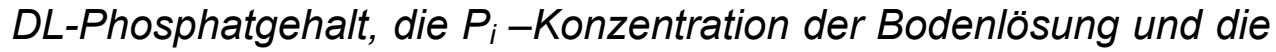
pH-Werte des humosen Sandbodens 30 Tage nach der Düngerapplikation

\begin{tabular}{|c|c|c|c|c|c|c|c|}
\hline \multirow{2}{*}{\multicolumn{2}{|c|}{ P-Form }} & \multicolumn{2}{|c|}{ DL-Phosphat } & \multicolumn{2}{|c|}{$\mathrm{P}_{\mathrm{i}}$-Konzentration } & \multicolumn{2}{|c|}{$\mathrm{pH}\left(\mathrm{CaCl}_{2}\right)$} \\
\hline & & $\mathrm{mg} \mathrm{P} \mathrm{kg}{ }^{-1}$ & relativ & $\mu g \mathrm{PL}^{-1}$ & relativ & absolut & relativ \\
\hline \multicolumn{2}{|l|}{ ohne } & 38 & 100 & 28 & 100 & 5,4 & 100 \\
\hline \multicolumn{2}{|l|}{ TSP } & 54 & 142 & 59 & 211 & 5,4 & 100 \\
\hline \multicolumn{2}{|c|}{ Hyper-P } & 54 & 142 & 27 & 96 & 5,5 & 102 \\
\hline KS 1 & Dollbergen & 56 & 147 & 9 & 32 & 6,3 & 117 \\
\hline KS 2 & Göttingen & 56 & 147 & 17 & 61 & 6,3 & 117 \\
\hline KS 3 & Gronau & 63 & 166 & 29 & 104 & 6,4 & 119 \\
\hline KS 4 & Hameln & 57 & 150 & 31 & 111 & 6,0 & 111 \\
\hline KS 5 & Einbeck & 53 & 140 & 23 & 82 & 5,5 & 102 \\
\hline KS 6 & Herzberg & 54 & 142 & 30 & 107 & 6,0 & 111 \\
\hline KS 7 & Gütersloh & 61 & 161 & 26 & 93 & 6,2 & 115 \\
\hline KS 8 & Northeim & 62 & 163 & 21 & 75 & 6,3 & 117 \\
\hline KS 9 & Uelzen & 53 & 140 & 34 & 121 & 5,4 & 100 \\
\hline KS 10 & Osterode & 56 & 147 & 27 & 96 & 5,9 & 109 \\
\hline KS 11 & Salzhausen & 57 & 150 & 34 & 121 & 5,6 & 104 \\
\hline KS 12 & Uetze & 63 & 166 & 28 & 100 & 6,5 & 120 \\
\hline KS 13 & Schöppenst. & 57 & 150 & 38 & 136 & 5,5 & 108 \\
\hline \multicolumn{2}{|l|}{$\mathrm{KS} \varnothing$} & 58 & 152 & 27 & 95 & 6,0 & 111 \\
\hline \multicolumn{2}{|l|}{ Fe 1} & 41 & 106 & 24 & 86 & 5,5 & 102 \\
\hline \multicolumn{2}{|l|}{ Fe 2} & 41 & 106 & 25 & 89 & 5,5 & 102 \\
\hline \multicolumn{2}{|l|}{ Ca 1} & 43 & 113 & 21 & 75 & 6,1 & 113 \\
\hline \multicolumn{2}{|l|}{ Ca 2} & 42 & 108 & 23 & 82 & 5,7 & 106 \\
\hline \multicolumn{2}{|l|}{$\mathrm{Fe} / \mathrm{Ca}$} & 43 & 113 & 8 & 29 & 6,1 & 113 \\
\hline \multicolumn{2}{|c|}{ GD $_{5 \%}$, Tukey } & 3,6 & 9,5 & 3,2 & 11 & 0,1 & 2 \\
\hline
\end{tabular}


Tabelle 29: Einfluss der P-Mineral- und Klärschlammdüngung (KS) auf die $P$ Sorption, die Bodengehalte an oxalatlöslichem $\mathrm{Fe}\left(\mathrm{Fe}_{\mathrm{ox}}\right)$ und Phosphor $\left(P_{o x}\right)$ des humosen Sandbodens 30 Tage nach der Düngerapplikation

\begin{tabular}{|c|c|c|c|c|c|c|c|}
\hline \multirow[t]{2}{*}{ P-Forn } & & \multicolumn{2}{|c|}{ P-Sorption } & \multicolumn{2}{|c|}{$\mathrm{Fe}_{\mathrm{ox}}$} & \multicolumn{2}{|c|}{$P_{\text {ox }}$} \\
\hline & & $\mathrm{mg} / \mathrm{kg}$ & relativ & $\mathrm{mg} / \mathrm{kg}$ & relativ & $\mathrm{mg} / \mathrm{kg}$ & relativ \\
\hline ohne & & 66 & 100 & 1706 & 100 & 319 & 100 \\
\hline TSP & & 62 & 94 & 1489 & 87 & 301 & 94 \\
\hline Hyper-F & & 69 & 105 & 1543 & 91 & 294 & 92 \\
\hline KS 1 & Dollbergen & 81 & 123 & 1749 & 103 & 308 & 97 \\
\hline KS 2 & Göttingen & 82 & 124 & 1699 & 100 & 320 & 100 \\
\hline KS 3 & Gronau & 80 & 121 & 1538 & 90 & 292 & 91 \\
\hline KS 4 & Hameln & 74 & 112 & 1629 & 96 & 329 & 103 \\
\hline KS 5 & Einbeck & 68 & 103 & 1560 & 92 & 293 & 92 \\
\hline KS 6 & Herzberg & 72 & 109 & 1650 & 97 & 340 & 107 \\
\hline KS 7 & Gütersloh & 80 & 121 & 1559 & 92 & 316 & 98 \\
\hline KS 8 & Northeim & 76 & 115 & 1533 & 90 & 307 & 95 \\
\hline KS 9 & Uelzen & 70 & 106 & 1637 & 96 & 316 & 98 \\
\hline KS 10 & Osterode & 69 & 105 & 1520 & 89 & 299 & 94 \\
\hline KS 11 & Salzhausen & 71 & 108 & 1627 & 95 & 316 & 98 \\
\hline KS 12 & Uetze & 76 & 115 & 1441 & 85 & 288 & 91 \\
\hline KS 13 & Schöppenst. & 73 & 111 & 1568 & 92 & 323 & 102 \\
\hline $\mathrm{KS} \varnothing$ & & 75 & 113 & 1587 & 93 & 311 & 97 \\
\hline $\mathrm{Fe} 1$ & & 71 & 108 & 1734 & 102 & 292 & 91 \\
\hline $\mathrm{Fe} 2$ & & 71 & 108 & 1878 & 110 & 333 & 105 \\
\hline Ca 1 & & 77 & 117 & 1558 & 91 & 289 & 91 \\
\hline Ca 2 & & 72 & 109 & 1568 & 92 & 304 & 95 \\
\hline $\mathrm{Fe} / \mathrm{Ca}$ & & 83 & 126 & 1707 & 100 & 290 & 91 \\
\hline $\mathrm{GD}_{5 \%}$ & ukey & 3,8 & 6 & 259 & 15 & 46 & 14 \\
\hline
\end{tabular}


Tabelle 29 gibt die Werte der P-Sorption wieder. Wie erwartet, reduzierte eine Triplephosphatgabe die P-Sorption und zwar auf $94 \%$. Durch die KS stieg die P-Sorption an. In Mittel waren es $13 \%$. Die Höchstwerte zeigten die KS 1, 2, 3 und 7 mit Werten von größer $120 \%$. Eine Eisenhydroxidzugabe erhöhte die PSorption um ca. $8 \%$, die mit Kalk um bis zu $17 \%$ und die Kombination um 26 $\%$, was nach einem additiven Effekt aussieht.

Die Werte der Oxalatextraktion für Fe und $\mathrm{P}$ (Tabelle 29) ergaben nur wenige signifikante Differenzen zwischen den Gehalten der Böden an $\mathrm{Fe}_{\mathrm{ox}}$ und $\mathrm{P}_{\mathrm{ox}}$. TSP reduzierte $\mathrm{Fe}_{\text {ox }}$ um $13 \%$, eine Kalkung um ca. 8 - $9 \%$ (Ca 1, Ca 2). Beide Differenzen waren aber im Vergleich zur Kontrolle nicht signifikant. Die KS 1 und 2 bewirkten signifikant höhere $\mathrm{Fe}_{\mathrm{ox}}$ - Werte als KS 12 .

\subsubsection{Beziehungen zwischen den P- Entzügen und Bodenkenngrößen bzw. zwischen verschiedenen Bodenkenngrößen (Regressions- analysen)}

Um zu erkennen, welche durch die variierte Düngung veränderten Kenngrößen des Versuchsbodens Einfluss auf die P- Entzüge der Pflanzen nehmen, wenn sie mit KS gedüngt werden, wurden Regressionsanalysen durchgeführt. Die folgenden Abbildungen (Abbildung 10, 11, 12, 13, 14 und 15) zeigen die Resultate.

Abbildung 10 zeigt die Abhängigkeit des P- Entzuges vom DL- P- Gehalt des Bodens nach KS- Düngung. Man erkennt, dass die DL- P- Werte nur zwischen etwa 53 und $63 \mathrm{mg} \mathrm{P} / \mathrm{kg}$ liegen. Diese Differenz von $10 \mathrm{mg} \mathrm{P} / \mathrm{kg}$ hatte keinen Einfluss auf die P- Entzüge.

Aus Abbildung 11 geht eindeutig hervor, die Anwendung der $13 \mathrm{KS}$ hatte zu stark differenzierten $\mathrm{P}_{\mathrm{i}}$ - Werten (ca. 8 - $38 \mu \mathrm{g} \mathrm{P} / \mathrm{I}$ ) geführt. Der P- Entzug der Pflanzen hing stark $r^{2}=0,67(r=0,82)$ von den $P_{i}$ - Werten ab, das heißt, mit dem Steigen der Orthophosphatkonzentration in der Bodenlösung nahm die PAufnahme der Pflanzen zu. 
Abbildung 12 zeigt die Abhängigkeit des P- Entzuges vom PSorptionsverhalten der Böden. Der Zusammenhang ist negativ, denn mit steigender P- Sorption sinkt die P- Aufnahme ab. Der Zusammenhang ist aber mit $r^{2}=0,33(r=0,57)$ lockerer als mit den $P_{i}$ - Werten.

Interessant in diesen Zusammenhang ist, dass der P- Entzug auch negativ vom Fe $e_{\text {ox }}$ Gehalt des Bodens beeinflusst wird (Abbildung 13). Hier war $r^{2}=0,44(r=$ $0,66)$ für den Zusammenhang sogar größer als bei dem Parameter P-Sorption.

Der Zusammenhang zwischen P- Entzug und Boden- pH-Werten (Abbildung 14) war im Bereich 5,5 bis 6,5 sehr locker $r^{2}=0,14$. Ohne den niedrigsten Wert für den P- Entzug (KS 1) wäre kein Trend zu erkennen.

Die Abbildung 15 zeigt die Abhängigkeit des $\mathrm{P}$ - Entzuges vom $\mathrm{Fe}$ : PVerhältnis der eingesetzten Klärschlämme. Das Bestimmtheitsmaß mit $r^{2}=0,86$ ist hoch $(r=0,93)$. Damit erhält diese Eigenschaft der KS eine große Bedeutung für die P- Verfügbarkeit der $\mathrm{KS}$, zumindest für den benutzten Sandboden.

Die Tabelle 30 zeigt zusammenfassend die Bestimmtheitsmaße für die linearen Regressionen zwischen P- Entzug und den geprüften Kenngrößen.

Tabelle 30: Bestimmtheitsmaße für 6 lineare Regressionen

\begin{tabular}{clccc}
\hline \multicolumn{1}{c}{$\mathrm{Y}$} & $\mathrm{X}$ & Zusammenhang & $\mathrm{r}^{2}$ & $\mathrm{r}$ \\
\hline P- Entzug & $\mathrm{DL}-\mathrm{P}$ & positiv & 0,02 & $0,14^{\mathrm{ns}}$ \\
& $\mathrm{P}_{\mathrm{i}}$ & positv & 0,67 & $0,82^{* * *}$ \\
& P-Sorp. & negativ & 0,33 & $0,58^{*}$ \\
& Fe & negativ & 0,44 & $0,66^{* *}$ \\
& $\mathrm{pH}$ & negativ & 0,14 & $0,37^{\text {ns }}$ \\
& Fe : P-Verh. der KS & negativ & 0,86 & $0,92^{* * *}$ \\
\hline
\end{tabular}

Statistische Sicherungen: nach E. Weber, 1972
$\mathrm{ns}=$ nicht signifikant
* = signifikant bei $p=5 \%$
** $=$ signifikant bei $p=1 \%$
$* * *=$ signifikant bei $p=0,1 \%$ 
Die engsten positiven Beziehungen bestehen zur $\mathrm{P}_{\mathrm{i}}$ - Konzentration der Bodenlösung $\left(r^{2}=0,67\right)$ und die engsten negativen zum Fe : P-Verhältnis des $\mathrm{KS}\left(\mathrm{r}^{2}=0,86\right)$.

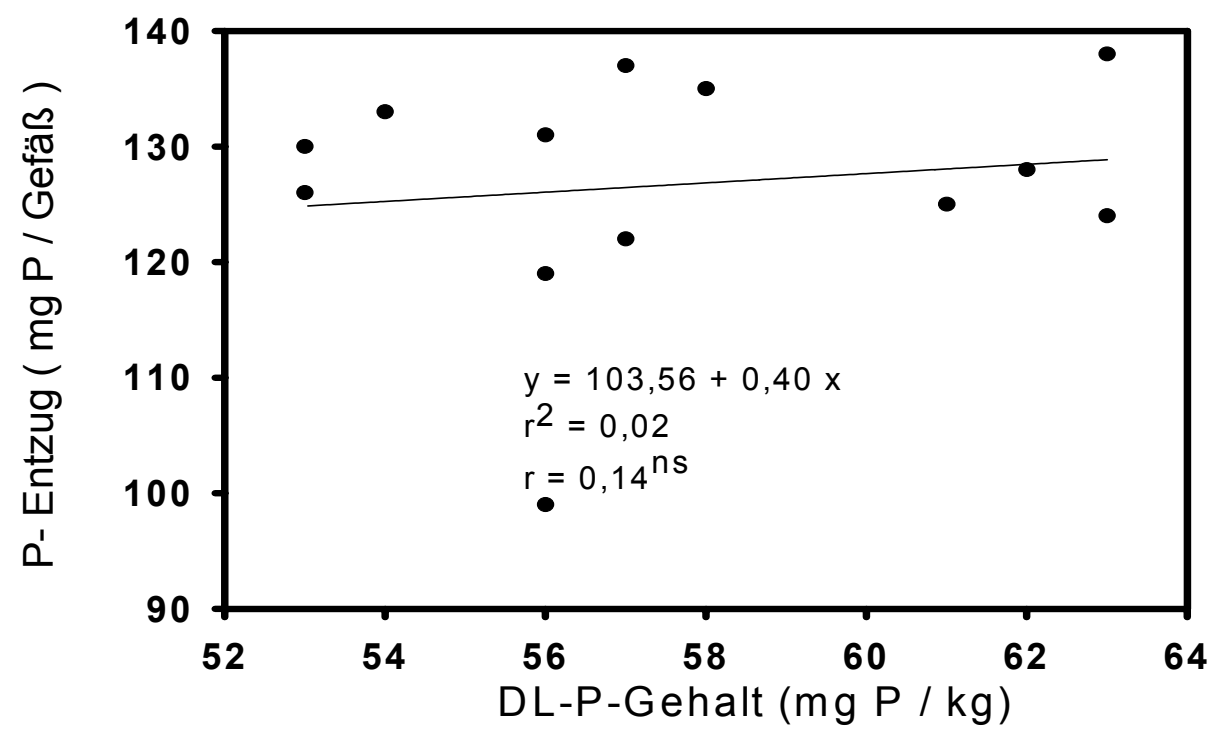

Abbildung 10: Abhängigkeit des $P$-Entzuges des Weidelgrases vom DL-P-Gehalt des Bodens, 30 Tage nach KS-Applikation $n s=$ nicht signifikant

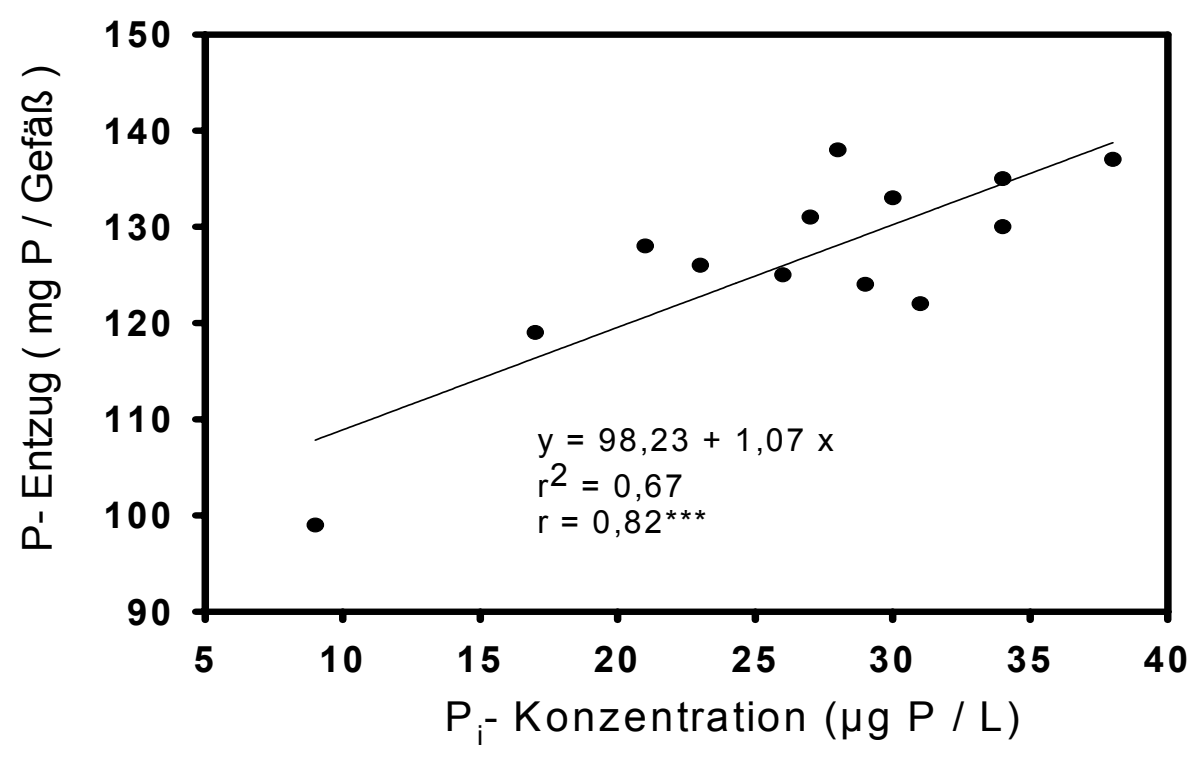

Abbildung 11: Abhängigkeit des P-Entzuges des Weidelgrases von der $P_{i^{-}}$Konzentration der Bodenlösung, 30 Tage nach KS-Applikation

${ }^{* * *}$ signifikant bei $p=0,1 \%$ 


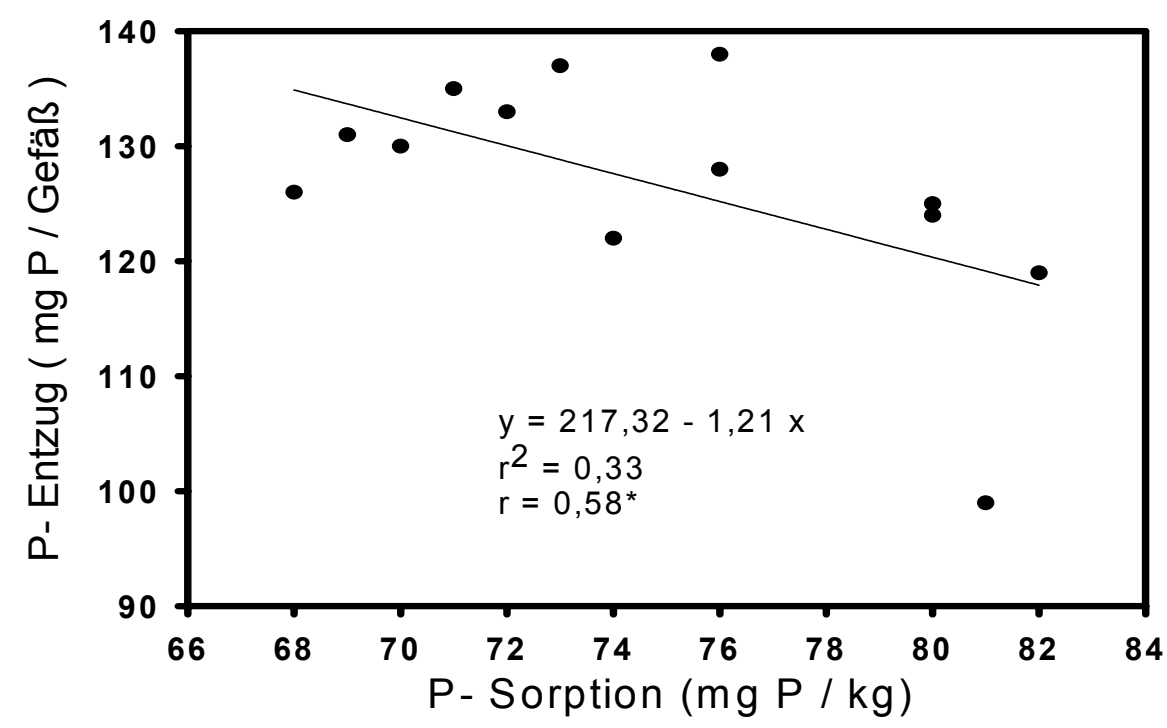

Abbildung 12: Abhängigkeit des $P$ - Entzuges des Weidelgrases von der P-Sorption des Bodens, 30 Tage nach KS-Applikation

* $=$ signifikant bei $p=5 \%$

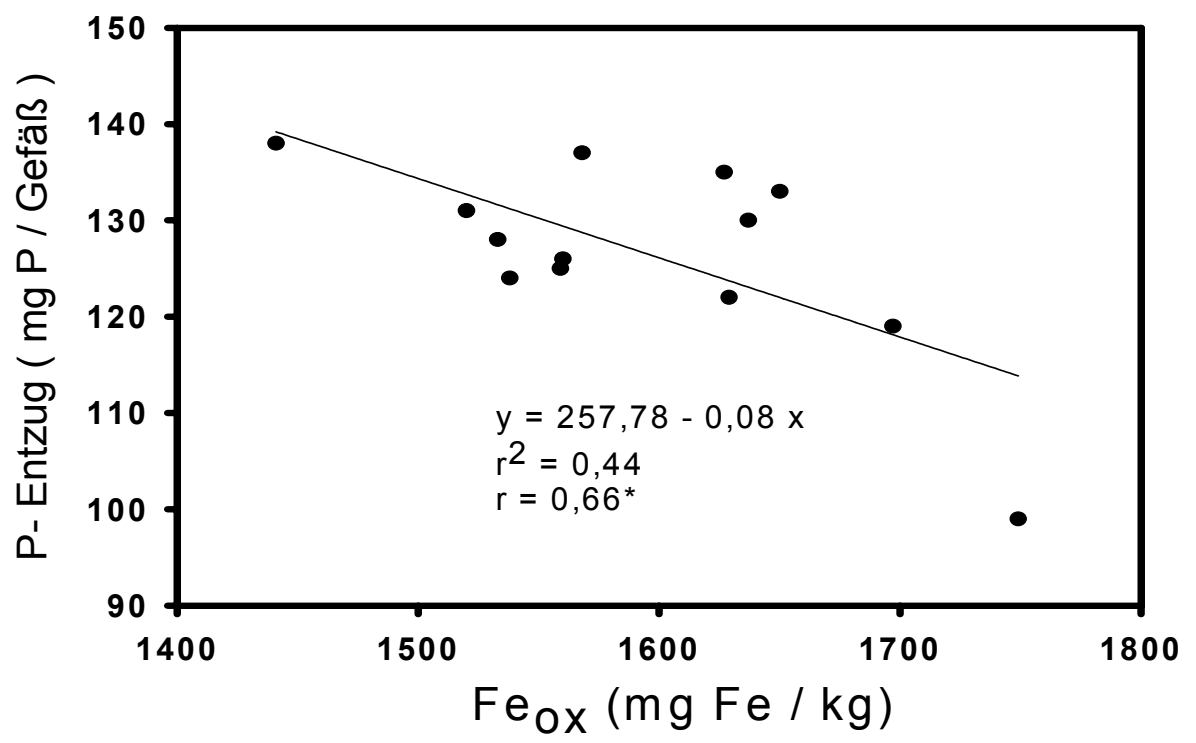

Abbildung 13: Abhängigkeit des P-Entzuges des Weidelgrases vom $\mathrm{Fe}_{\text {ox }}-$ Gehalt des Bodens, 30 Tage nach KS-Applikation ${ }^{*}=$ signifikant bei $p=5 \%$ 


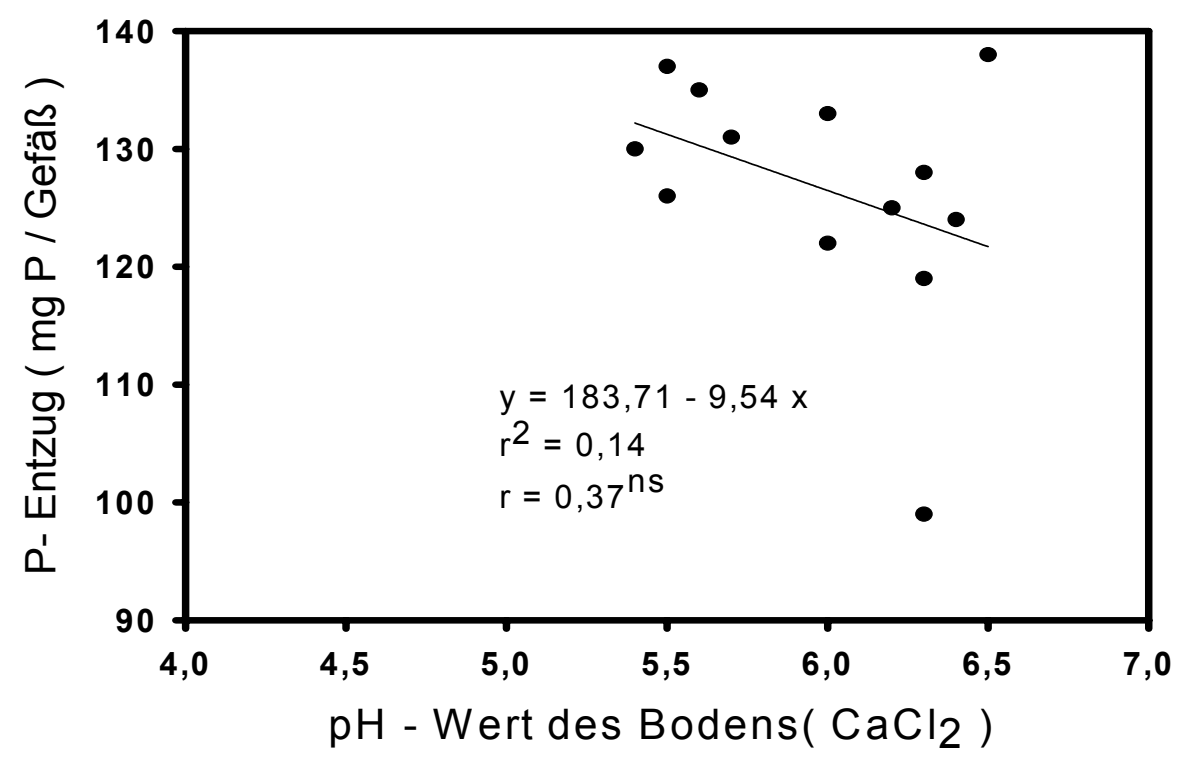

Abbildung 14: Abhängigkeit des P-Entzuges des Weidelgrases vom $\mathrm{pH}$ des Bodens, 30 Tage nach KS-Applikation $n s=$ nicht signifikant

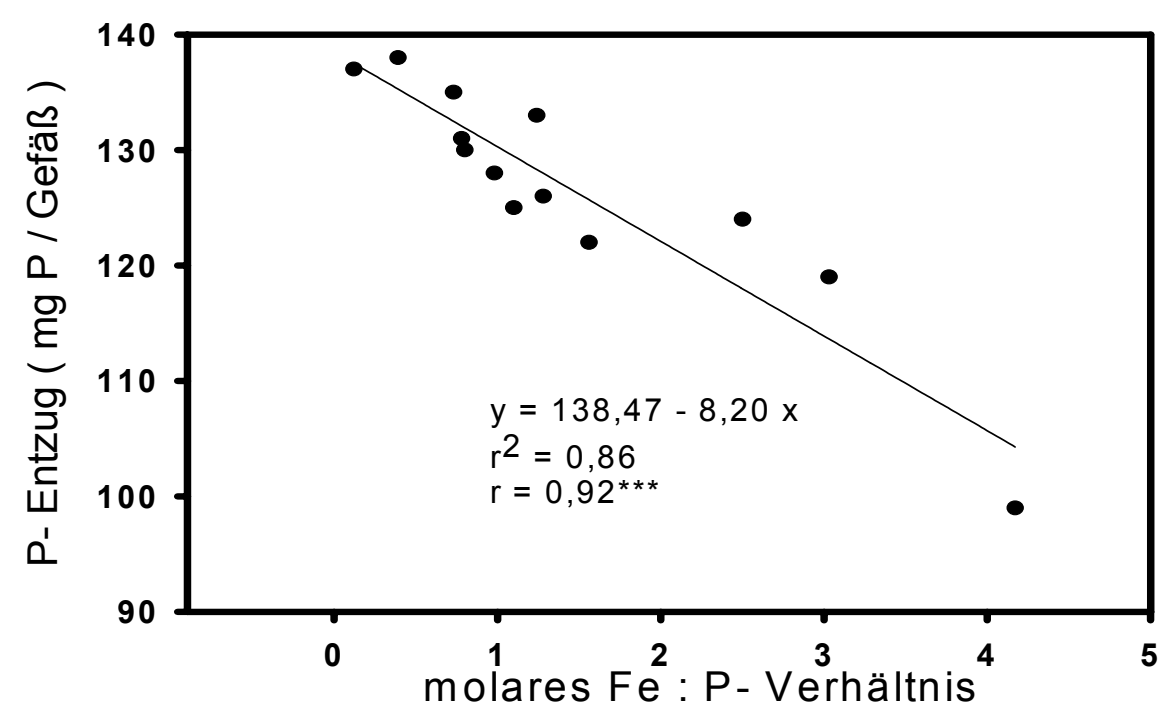

Abbildung 15: Abhängigkeit des P-Entzuges des Weidelgrases vom molaren $F e$ : P-Verhältnis der Klärschlämme

$$
{ }^{* * *}=\text { signifikant bei } p=0,1 \%
$$


Die folgenden Abbildungen (Abbildung 16, 17, 18, 19, 20, 21, 22, und 23) zeigen die Ergebnisse von Regressionsanalysen zwischen den verschiedenen Bodenkenngrößen, um eventuelle Zusammenhänge zwischen ihnen zu erkennen. Sie könnten bedeutsam sein für die P-Aufnahme der Pflanzen.

Abbildung 16 zeigt den Zusammenhang zwischen zwei Kenngrößen des Bodens, die für die Beurteilung der P- Verfügbarkeit genutzt werden. Man erkennt, dass bei DL-P- Gehalten zwischen ca. 53 und $63 \mathrm{mg} \mathrm{P} \mathrm{/} \mathrm{kg} \mathrm{Boden} \mathrm{die}$ $\mathrm{P}_{\mathrm{i}}$ - Werte keine Tendenz einer Abhängigkeit zeigen.

Anders ist das Verhältnis zwischen $\mathrm{P}_{\mathrm{i}}$ - Werten und $\mathrm{P}$ - Sorption (Abbildung 17). Hier ist ein klarer Trend zu erkennen. Mit steigender $\mathrm{P}$ - Sorption sinken die $\mathrm{P}_{\mathrm{i}}$ Werte ab.

Die Abbildungen 18 und 19 zeigen die Abhängigkeit der $\mathrm{P}_{\mathrm{i}}$ - Konzentration vom $\mathrm{Fe}_{\text {ox }}-$ Gehalt des Bodens bzw. vom Boden- pH. Beide Größen führen bei ansteigenden Werten zu einem Absinken der $\mathrm{P}_{\mathrm{i}}$ - Konzentration. Dabei ist der Zusammenhang mit den $\mathrm{pH}-$ Werten offenbar enger $\left(\mathrm{r}^{2}=0,29\right)$ als mit den $\mathrm{Fe}_{\mathrm{ox}}{ }^{-}$ Werten $\left(r^{2}=0,15\right)$.

Abbildung 20 zeigt, inwieweit die $\mathrm{P}_{\mathrm{i}}$ - Konzentration direkt vom $\mathrm{Fe}$ : P-Verhältnis der KS abhängig ist. Dieser Zusammenhang ist relativ eng $\left(r^{2}=0,58\right)$. Das heißt, mit weiten molaren Fe: $\mathrm{P}$ - Verhältnissen sinken die $\mathrm{P}_{\mathrm{i}}$ - Werte stark ab. Also ist z.B. im Boden mit KS 1 (Dollbergen) mit dem weitesten Fe: PVerhältnis von 4,2 (ca. $1: 0,24)$ (vgl. Tabelle 20) der $\mathrm{P}_{\mathrm{i}}$ - Wert am geringsten.

Die Orthophosphatkonzentration ( $\mathrm{P}_{\mathrm{i}}$ - Konz.) hängt, wie zu sehen ist, von den Größen wie $\mathrm{Fe}_{o x}$ und $\mathrm{pH}$ bzw. direkt vom $\mathrm{Fe}$ : P- Verhältnis der KS ab. Diese Größen regulieren aber auch die P- Sorption, die letztlich über die P- Pufferung die Höhe der $\mathrm{P}_{\mathrm{i}}$ - Werte mitbestimmt. Deshalb wurden auch die Beziehungen der P-Sorption zu einigen Bodenkenngrößen untersucht.

Aus Abbildung 21 geht hervor, dass die Fe ox $^{-W e r t e ~ v o n ~ c a . ~} 1450$ bis $1750 \mathrm{mg}$ $\mathrm{kg}^{-1}$ durch die KS angestiegen sind, dass aber die Beziehung zur P-Sorption sehr locker ist $\left(r^{2}=0,06\right)$. Dagegen hatte der $\mathrm{pH}$ - Anstieg von ca. 5,5 auf 6,5 eine eindeutig positive Wirkung auf den Anstieg der P-Sorption $\left(r^{2}=0,68\right)$. 
Bemerkenswert ist ferner, dass mit einem weiteren Fe: P- Verhältnis in den KS ein deutlicher Anstieg der P- Sorption erfolgt (Abbildung 23).

Die Tabelle 31 zeigt zusammenfassend die Bestimmtheitsmaße für die linearen Regressionen zwischen den ausgewählten Kenngrößen.

Tabelle 31: Bestimmtheitsmaße zwischen verschiedenen Kenngrößen des Bodens bzw. der KS

\begin{tabular}{|c|c|c|c|c|}
\hline $\mathrm{Y}$ & $x$ & Zusammenhang & $r^{2}$ & $r$ \\
\hline \multirow[t]{5}{*}{ Pi- Konz. } & DL-P & Kein & 0,00 & $0,00^{\text {ns }}$ \\
\hline & P-Sorp. & negativ & 0,31 & $0,56^{\mathrm{ns}}$ \\
\hline & $\mathrm{Fe}_{\mathrm{ox}}$ & negativ & 0,15 & $0,39^{\text {ns }}$ \\
\hline & $\mathrm{pH}$ des Bodens & negativ & 0,29 & $0,54^{\mathrm{ns}}$ \\
\hline & Fe : P-Verh. des KS & negativ & 0,58 & $0,76^{* *}$ \\
\hline \multirow[t]{3}{*}{ P-Sorp. } & $\mathrm{Fe}_{\mathrm{ox}}$ & positiv & 0,06 & $0,25^{\text {ns }}$ \\
\hline & $\mathrm{pH}$ des Bodens & positiv & 0,68 & $0,83^{* * *}$ \\
\hline & Fe : P-Verh. des KS & positiv & 0,44 & $0,66^{*}$ \\
\hline
\end{tabular}

Statistisch: (nach E. Weber 1972)
ns = nicht signifikant
* = signifikant bei $p=5 \%$
** $=$ signifikant bei $p=1 \%$
${ }^{* * *}=$ signifikant bei $p=0,1 \%$ 


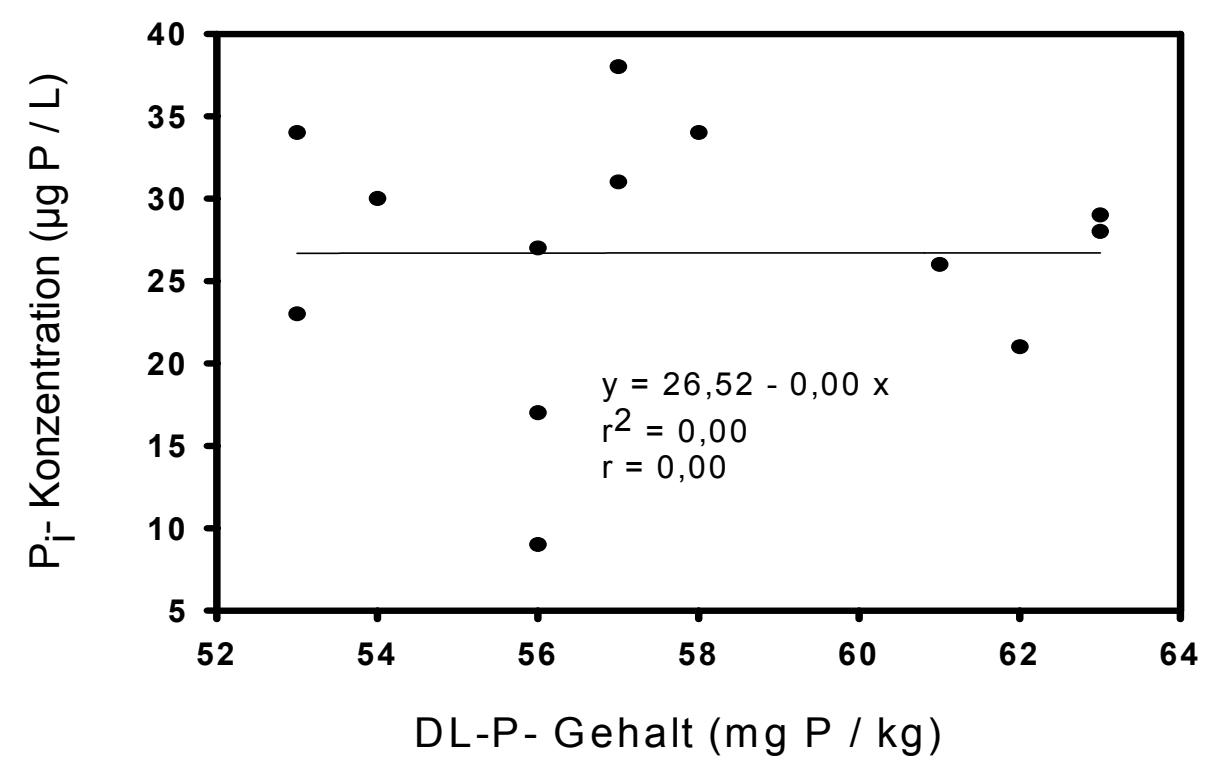

Abbildung 16: Abhängigkeit der $P_{i^{-}}$Konzentration vom $D L-P$ Gehalt des Bodens, 30 Tage nach KS-Applikation

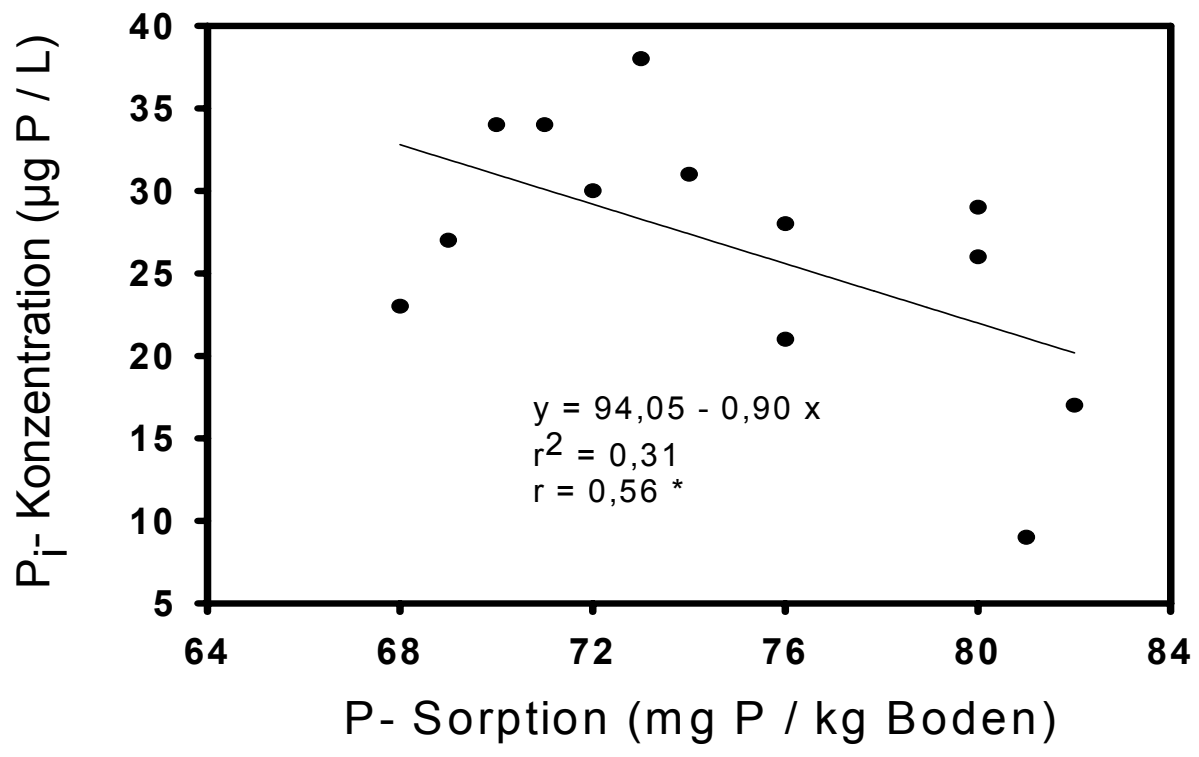

Abbildung 17: Abhängigkeit der $P_{i-}$ Konzentration von der $P$ - Sorption des Bodens nach KS-Applikation * $=$ signifikant bei $p=5 \%$ 


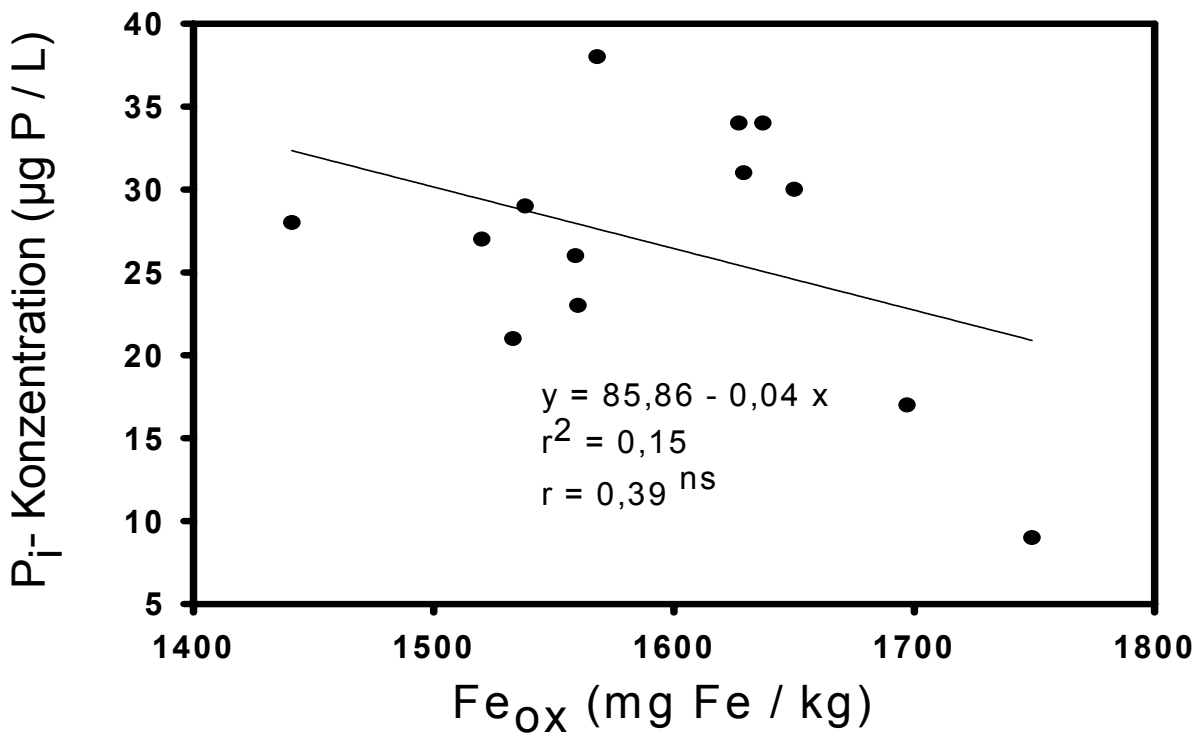

Abbildung 18: Abhängigkeit der $P_{i-}$ Konzentration vom Bodengehalt an $\mathrm{Fe}_{\mathrm{ox}}, 30$ Tage nach KS-Applikation $n s=$ nicht signifikant

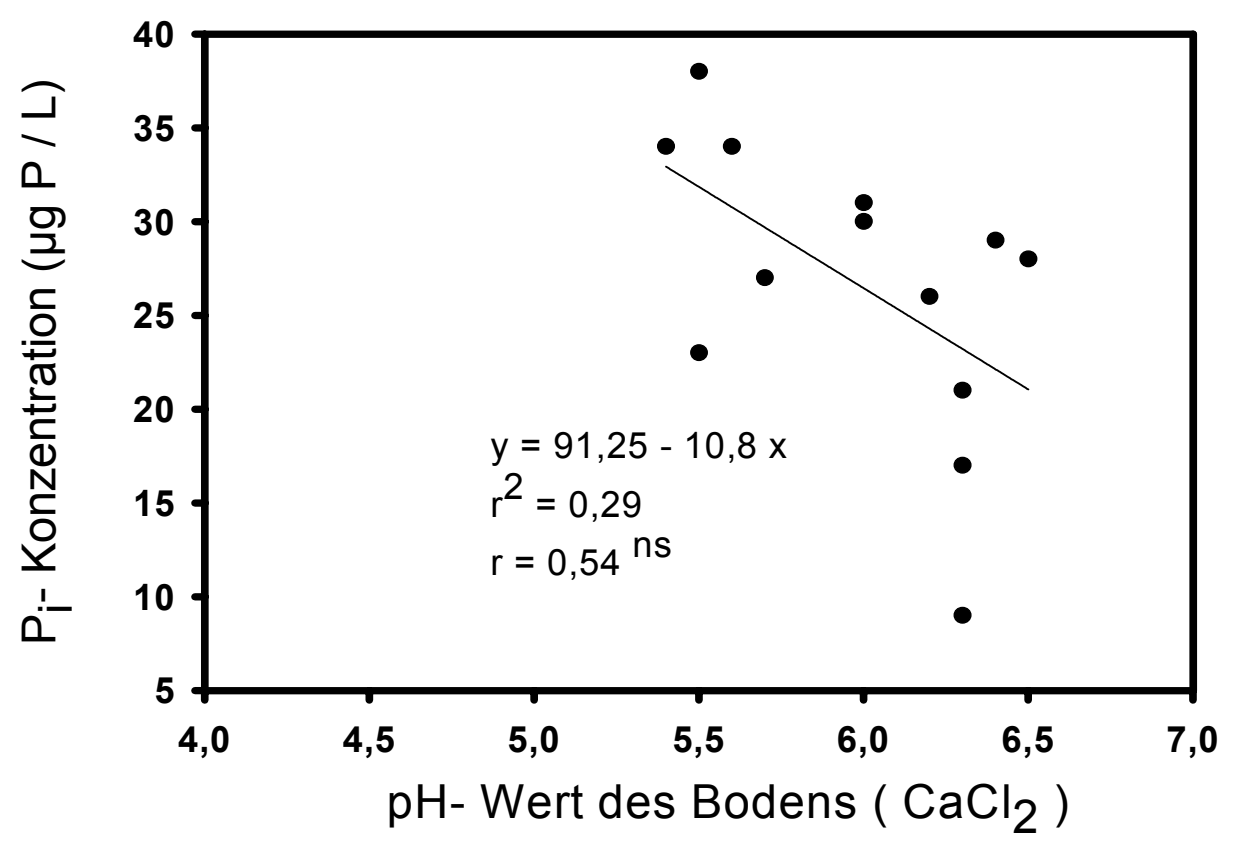

Abbildung 19: Abhängigkeit der $P_{i^{-}}$Konzentration in der Bodenlösung vom Boden- $\mathrm{pH}, 30$ Tage nach KS-Applikation $n s=$ nicht signifikant 


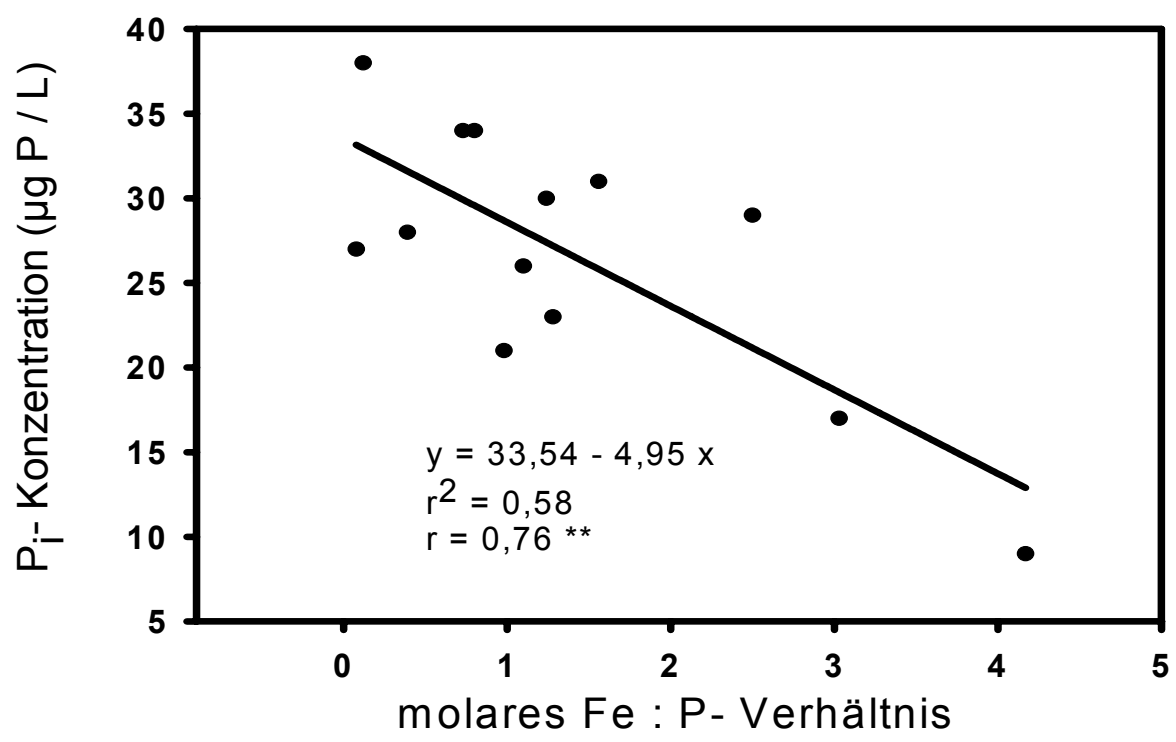

Abbildung 20: Abhängigkeit der $P_{i}$-Konzentration vom molaren $\mathrm{Fe}: \mathrm{P}$-Verhältnis der Klärschlämme ${ }^{* *}=$ signifikant bei $p=1 \%$

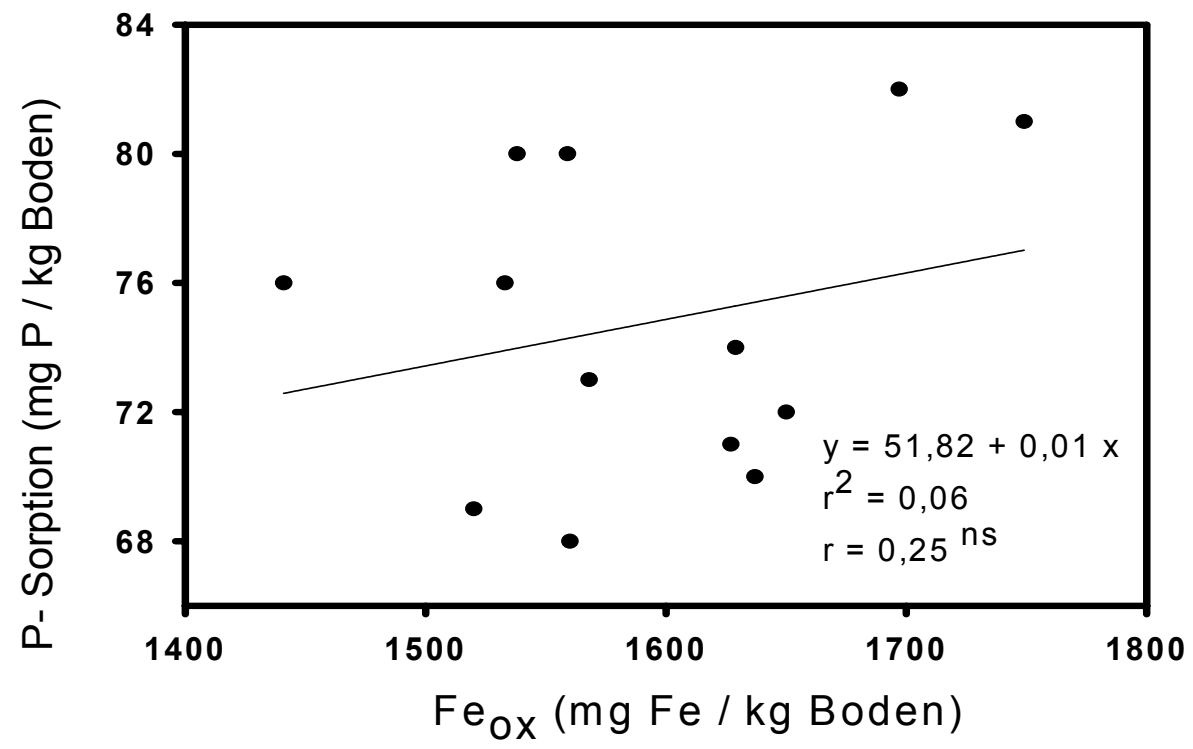

Abbildung 21: Abhängigkeit der P-Sorption vom Fe ox $^{-G e h a l t ~}$ des Bodens, 30 Tage nach KS-Applikation $n s=$ nicht signifikant 


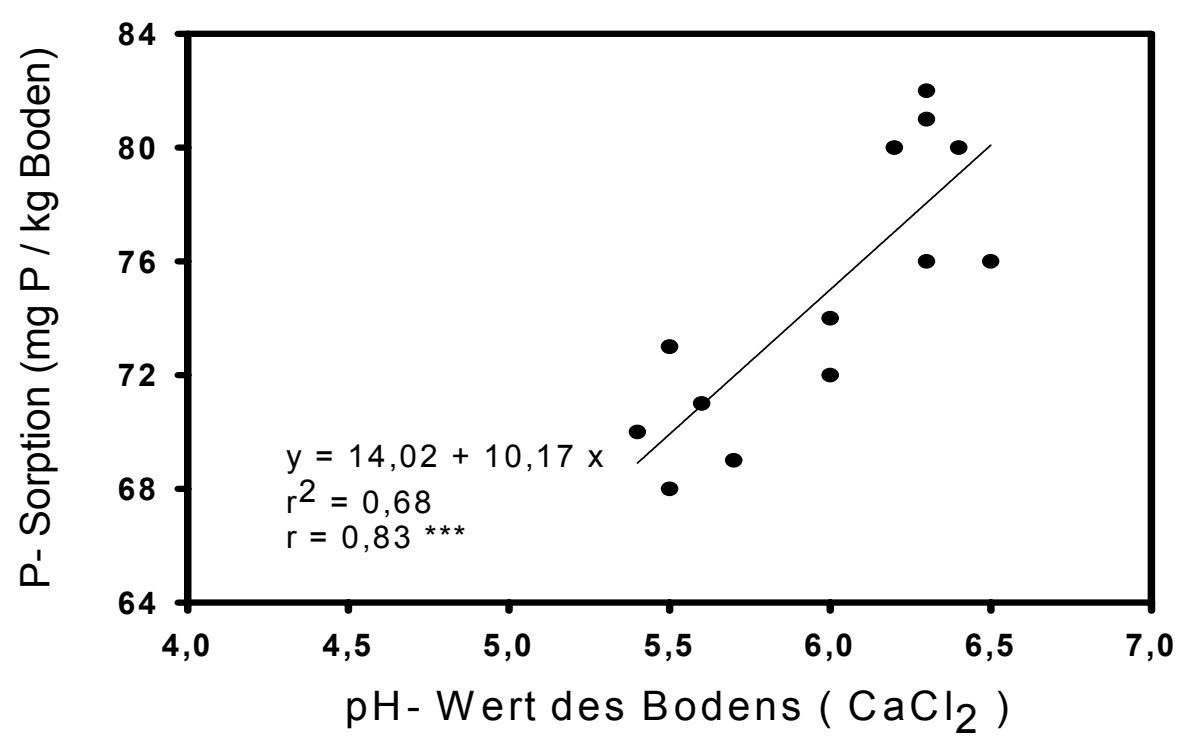

Abbildung 22: Abhängigkeit der P-Sorption vom BodenpH nach der KS-Applikation

$* * *=$ signifikant bei $p=0,1 \%$

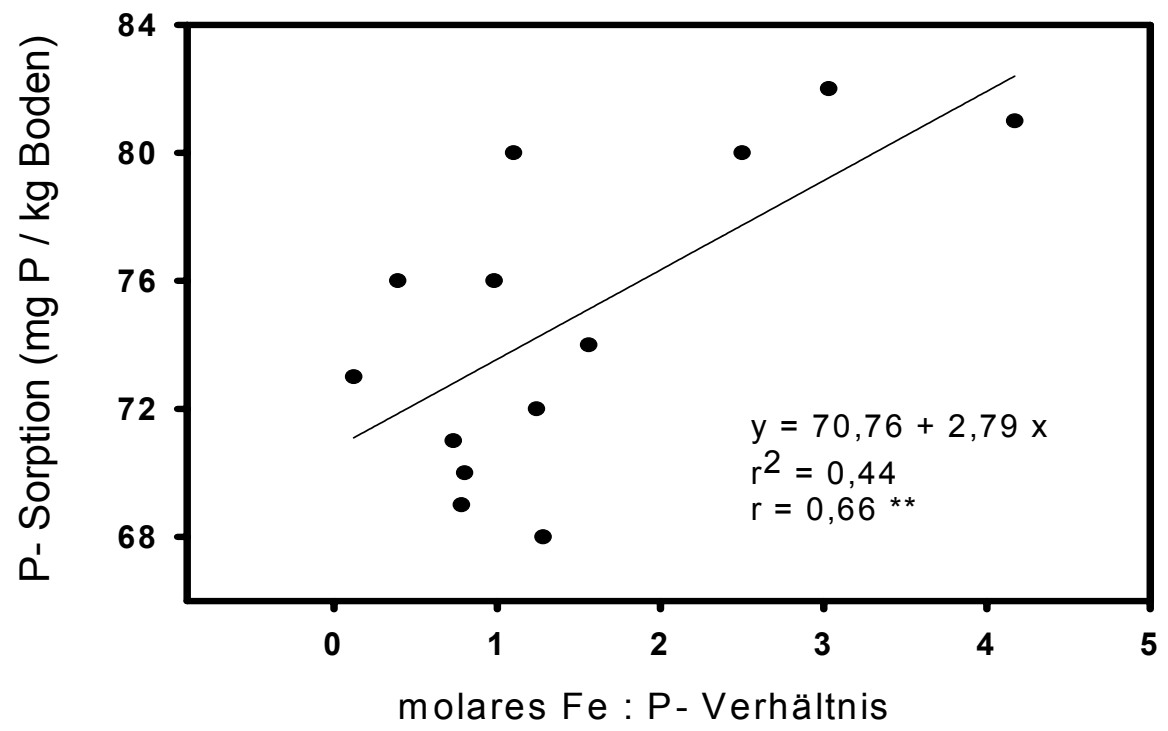

Abbildung 23: Abhängigkeit der P-Sorption vom molaren $\mathrm{Fe}:$ P-Verhältnis der Klärschlämme ${ }^{* *}=$ signifikant bei $p=1 \%$ 


\subsubsection{Diskussion}

Der P-Entzug des Weidelgrases aus den 13 Klärschlämmen war sehr differenziert. Bezogen auf das aus TSP aufnehmbare $P$ wurden von 6 Klärschlämmen (KS) 82 bis $100 \%$, von 5 KS nur 63 bis $81 \%$ und von 2 KS nur weniger als $62 \%$ des applizierten $\mathrm{P}$ aufgenommen. Dieses differenzierte Verhalten der KS dürfte, da nur ein Boden benutzt wurde, im Wesentlichen auf die Eigenschaften der KS zurückzuführen sein. Sie beeinflussen mit ihren Phosphaten, ihren Eisenverbindungen, aber auch ihren basischen Bestandteilen die P-Löslichkeit in der Bodenlösung und die P-Sorption. Beide Größen sind für die P-Aufnahme der Pflanzen verantwortlich. Mit steigendem $\mathrm{Fe}_{\mathrm{ox}^{-}}$Gehalt und steigender Kalkzufuhr sank die Pi-Konzentration ab (Abbildung 18), am besten demonstriert durch die Abhängigkeit vom Fe:PVerhältnis der KS selbst (Abbildung 20). Für die P- Sorption gilt die umgekehrte Aussage: Mit steigenden $\mathrm{Fe}_{\mathrm{ox}}-$ Gehalten und steigender Kalkzufuhr stieg die PSorption an, besonders infolge der Kalkzufuhr (Abbildung 21). Sie stieg aber auch mit dem Weiterwerden des Fe: P- Verhältnisses (Abbildung 23).

Es ist bemerkenswert, dass diese Resultate bereits mit einer einzigen KS-Gabe in Höhe von nur 5 t TM ha-1 (bezogen auf den KS 2 (Göttingen) erfolgte, also einer Gabe wie sie in der Praxis angewendet wird. In Teil I der Arbeit waren KSGaben auch in doppelter (10 t) bzw. in 3-facher Höhe (15 t) appliziert worden. Die P-Sorption war dort linear mit dem $\mathrm{Fe}_{\mathrm{ox}}-$ Gehalt angestiegen. Auf dem sehr ähnlichen Boden Düshorn (Brache) betrug dort (Abb. 9) $r^{2}$ für die Beziehung zwischen diesen zwei Kenngrößen 0,92. Die Beziehung zwischen P- Sorption und oxalatlöslichem Eisen war also eng. Wenn infolge steigender P-Sorption, die $\mathrm{P}_{\mathrm{i}}$ - Konzentration niedriger wird, so wird verständlich, dass damit die $\mathrm{P}$ Aufnahme der Pflanzen ebenfalls erniedrigt wird. Damit werden folgende Literaturbefunde bestätigt bzw. plausibel:

Mit eisenhaltigem KS stieg die P-Sorption im Boden an (Fritsch und Werner, 1989). Die P-Ausnutzung von eisenhaltigem KS im Vergleich zu Ca $\left(\mathrm{H}_{2} \mathrm{PO}_{4}\right)_{2}$ lag nur bei 62 \% (Frossard, E., et al., 1996). Suntheim und Dittrich (1996) fanden beim Vergleich von Superphosphat mit $3 \mathrm{KS}$ verschieden starker PEliminierung eine deutlich geringere P-Aufnahme von Mais, wenn Fe- Salze 
zur P- Eliminierung verwendet worden waren. Auch Weidelgras, Raps und Buchweizen konnten aus KS mit P- Eliminierung durch Eisensalze bzw. Eisensalze mit $\mathrm{CaO}$ soviel $\mathrm{P}$ aufnehmen wie aus Superphosphat (Baran, 1985). Das In-Lösung-gehen von $\mathrm{P}$ aus $\mathrm{KS}$ mit 7 \% Fe war deutlich geringer als aus Fällungsprodukten mit Kalk. Außerdem war die P-Aufnahme aus den Fehaltigen KS gegenüber anderen deutlich geringer (Zorn, et al., 2001).

Wenn also der Eisengehalt der KS eine Rolle für die P-Verfügbarkeit im Boden spielt, so stellt sich die Frage, welcher Fe- Gehalt toleriert werden kann, wenn die P-Ausnutzung möglichst der von Triplephosphat entsprechen sollte. Aus Tabelle 26 geht hervor, dass die P- Entzugswerte (82 - $100 \%$ ) aus den Klärschlämmen 9 bis 13 nicht signifikant von der aus TSP verschieden sind. KS 6 bildet einen Sonderfall, dessen relativ hohe PAusnutzung (89 \%) die sonst klare Einteilung der KS (entsprechend ihrer Fe: PVerhältnisse in die 3 Gruppen) etwas stört. Die Ursache ist unklar. KS 6 wird nicht weiter berücksichtigt.

Die Schlämme 9 bis 13 haben molare Fe: P- Verhältnisse von weiter als 1:1,26, sind also zu Gunsten des P verschoben. D.h. in den KS 9 bis 11 sind die FeVerbindungen stöchiometrisch betrachtet mehr als 1-fach, im KS 12 fast vollständig und im KS $13 \mathrm{mehr}$ als vollständig mit Phosphat gesättigt. Interessant ist, dass mit den KS 12 und 13 die absolut gleichen P-Entzüge wie mit TSP erreicht werden (Tabelle 25). Dieser Befund spricht sehr dafür, die FeGehalte so niedrig wie möglich zu halten. Der umgekehrte Schluss ist, die KS 1 bis 8 enthalten zu viele Eisenverbindungen mit geringer P-Sättigung.

Ein Problem ist noch anzusprechen. Die Kalkgehalte der KS sind sehr verschieden (Tabelle 20) und nehmen somit differenzierenden Einfluss auf die P-Dynamik im Boden und auf das Wachstum der Pflanzen. Für das Wachstum (Sprossmasse) konnte allerdings keine Abhängigkeit von der $\mathrm{pH}-$ WertÄnderung infolge der KS-Zufuhr festgestellt werden. Das Bestimmtheitsmaß für diese Beziehung betrug $r^{2}=0,05$ ! In den Gefäßen der Varianten ohne Kalkzufuhr P 0, TSP, KS 5, 9, 11 und 13 werden pH-Werte von 5,4 bis 5,6 gemessen (Tabelle 28). Für einen Sandboden mit $1 \%$ Ton plus $7 \%$ Schluff ist dieser Wert nahe dem Optimum. Also ist durch eine Kalkzufuhr nur ein relativ 
geringer Ertragszuwachs zu erwarten. In den Varianten mit Klärschlamm, die hohe Kalkmengen enthielten, waren die $\mathrm{pH}$-Werte durch diese eine KSApplikation in gesetzlich zulässiger Höhe von ca. $5 \mathrm{t} \mathrm{TM} \mathrm{ha}^{-1}$ auf Werte von 6,3 bis 6,5 angestiegen! Im Jahr 2002, in dem der Versuch fortgeführt wurde, hier aber nicht mit betrachtet wird, trat in einigen KS- Varianten mit hohen $\mathrm{CaO}$ Begleitgaben (KS Göttingen, Dollbergen u.a.) die Dörrfleckenkrankheit bei Hafer auf. Das ist ein Hinweis auf Mn- Mangel. Dieser pH-Wert von 6,5 ist vermutlich für diesen Boden schon zu hoch, denn die durch eine Kalkdüngung erzielten höheren Trockenmassenerträge von 15 - 23\% (Tabelle 27) wurden bei pH-Werten von 5,7 bis 6,1 erzielt (Tabelle 28). Für Sandböden ist also die Kalkzufuhr durch Klärschlämme strikt zu kontrollieren, denn höhere pHWerte können dort zu Mikronährstoffmangel (z.B. Mangan) führen.

In Bezug auf die P-Dynamik hat sich gezeigt (Abbildung 19), dass die $\mathrm{P}_{\mathrm{i}}$ Konzentration mit steigendem $\mathrm{pH}$ (steigende Kalkzufuhr) sinkt und die $\mathrm{P}$ Sorption steigt (Abbildung 17). Offenbar geht hier das Phosphat Bindungen mit den $\mathrm{Ca}$ - Ionen ein bzw. wird an $\mathrm{CaCO}_{3}$ - Partikel adsorbiert. Diese Prozesse geschehen bei der KS- Herstellung und im Boden. Die Konsequenzen für die PAufnahme der Pflanzen sind aber offenbar gering, denn das Bestimmtheitsmaß für die Beziehung zwischen $\mathrm{P}$ - Entzug und $\mathrm{pH}$ ist mit $\mathrm{r}^{2}=0,14$ gering, aber mit $\mathrm{Fe}_{\mathrm{ox}}\left(\mathrm{r}^{2}=0,44\right)$ deutlich höher. Das kann bedeuten, dass das an CaVerbindungen gebundene Phosphat besser pflanzenverfügbar ist als das an Fe-Verbindungen gebundene.

\section{Schlussfolgerungen}

Enthalten Klärschlämme (KS) je 1 mol Fe (56 g) weniger als 1,3 mol P (40 g), so sinkt inre P- Ausnutzung signifikant unter die von Triplesuperphosphat ab.

- Solche Klärschlämme sind in der Landwirtschaft unerwünscht. Klärschämme sollten so wenig $\mathrm{Fe}$ enthalten wie das Verfahren erlaubt. Auf jeden Fall sollten bei der Schlammkonditionierung über die vorher erfolgte P-Fällung hinaus keine weiteren Eisensalze eingesetzt werden. 
Den Landwirten sind neben den Nährstoffgehalten, Schwermetallgehalten, basischen Bestandteilen auch die Eisengehalte und damit die $\mathrm{Fe}$ : PVerhältnisse in Absolutzahlen oder im molarem Verhältnis mitzuteilen.

- Bei Sandböden ist die Kalkzufuhr mit dem KS für die Einhaltung optimaler $\mathrm{pH}-$ Werte unbedingt zu beachten. 
Teil III

$4 \quad$ Kleingefäßversuch zur Prüfung der P-Aufnahme aus drei

Klärschlämmen mit unterschiedlichen Werten des molaren Fe: $\mathbf{P}$.

Verhältnisses auf zwei deutlich verschiedenen ägyptischen Böden

\subsection{Vorbemerkungen und Versuchsziele}

Zur Prüfung der P- Wirksamkeit von drei eisenhaltigen Klärschlämmen (Tabelle 32) wurden zwei ägyptische Böden (Tabelle 33) in einem Kleingefäßversuch (1 kg Boden) benutzt. Die Versuchspflanze war Mais. Der Versuch erfolgte in der Klimakammer.

Als Bezugsbasis wurde eine mineralische $\mathrm{P}$ - Düngung mit Triplesuperphosphat benutzt. Die drei Klärschlämme wurden so ausgewählt, dass das molekulare Fe: $P$ Verhältnis möglichst einen hohen, mittleren bzw. einen niedrigen Wert haben sollte, um die Wirksamkeit dieses Verhältnisses auf die P- Aufnahme von Pflanzen prüfen zu können. Die drei benutzten Klärschlämme (KS- Göttingen, KS- Dollbergen, KS- Osterode) stammen aus niedersächsischen Kläranlagen. Wichtige Kenndaten stehen in Tabelle 32.

Tabelle 32: Kenndatenanalysen der gefriergetrockneten, applizierten Klärschlämme

\begin{tabular}{lcccccc}
\hline \multicolumn{1}{c}{ Ort } & $\begin{array}{c}\mathrm{P} \\
\mathrm{mg} \mathrm{kg}\end{array}$ & $\begin{array}{c}\mathrm{Fe} \\
\mathrm{mg} \mathrm{kg}^{-1}\end{array}$ & $\begin{array}{c}\mathrm{Fe} / \mathrm{P} \\
\text { direkt }\end{array}$ & \multicolumn{2}{c}{$\mathrm{Mol}}$. & $\mathrm{Fe}: \mathrm{P}$ \\
\hline Dollbergen & 8777 & 65045 & $7,4: 1$ & $1: 0,24$ & $(4,2)$ & 235000 \\
Göttingen & 11354 & 61810 & $5,4: 1$ & $1: 0,33$ & $(3,0)$ & 271000 \\
Osterode & 30087 & 42050 & $1,4: 1$ & $1: 1,27$ & $(0,79)$ & 218000 \\
\hline
\end{tabular}

\subsection{Versuchsanlage und Durchführung}

Die verwendeten Böden aus Ägypten (Moschtohor und Meetkenana) entstammen landwirtschaftlich genutzten Ackerflächen aus 0-30 cm Tiefe. Der Boden aus Moschtohor ist ein lehmiger Tonboden, der aus Meetkenan ist ein 
toniger Sandboden. Wichtige Kenndaten der eingesetzten Böden gibt Tabelle 33 wieder. Die pH - Werte beider Böden liegen im basischen Bereich. Die CALP- Gehalte sind als hoch (Gehaltsklasse D des VDLUFA) zu bezeichnen.

Tabelle 33: Kenngrößen der ägyptischen Versuchsböden

\begin{tabular}{cccccccc}
\hline Ort & $\begin{array}{c}\text { Ton } \\
\%\end{array}$ & $\begin{array}{c}\text { Schluff } \\
\%\end{array}$ & $\begin{array}{c}\text { Sand } \\
\%\end{array}$ & $\begin{array}{c}\mathrm{CaCO}_{3} \\
\%\end{array}$ & $\begin{array}{c}\mathrm{pH} \\
\left(\mathrm{CaCl}_{2}\right)\end{array}$ & $\begin{array}{c}\text { Humus } \\
\%\end{array}$ & $\begin{array}{c}\mathrm{CAL}-\mathrm{P} \\
\mathrm{mg} \mathrm{P} \mathrm{kg}^{-1}\end{array}$ \\
\hline Moschtohor & 56 & 19 & 25 & 1,4 & 8,0 & 2,20 & 158 \\
Meetkenana & 14 & 8 & 78 & 0,6 & 7,7 & 1.98 & 106 \\
\hline
\end{tabular}

In die Böden wurden die gefriergetrockneten und fein gemahlenen Klärschlämme eingemischt. Zusätzlich wurden die Kontrollvarianten ohne PDüngung (P 0) angelegt. Eine Variante wurde mit Triplesuperphosphat (TSP) gedüngt. Vor dem Befüllen der Kleingefäße wurden in die jeweils $1 \mathrm{~kg}$ Boden die folgenden Nährstoffmengen eingearbeitet:

- $31 \mathrm{mg} \mathrm{P}$ als Triplesuperphosphat oder Klärschlamm in fester Form,

- $0,15 \mathrm{~g} \mathrm{~N}$ als $\mathrm{NH}_{4} \mathrm{NO}_{3}$,

- $\quad 0,15 \mathrm{~g} \mathrm{~K}$ als $\mathrm{K}_{2} \mathrm{SO}_{4}$ und

- $\quad 0,03 \mathrm{~g} \mathrm{Mg}$ als $\mathrm{MgSO}_{4} \cdot 7 \mathrm{H}_{2} \mathrm{O}$ in gelöster Form.

Die Varianten wurden in vierfacher Wiederholung angesetzt. Den Versuchsaufbau und das Versuchsdesign gibt Tabelle 34 wieder. Bei der Vorbereitung des Versuchs wurde der Boden in den Gefäßen auf ca. 50\% der max. Wasserkapazität eingestellt und abgedeckt. Nach 4 Wochen wurden sie mit Mais der frühreifen Sorte „BIRKO“ bepflanzt. Zunächst wurde das verwendete Saatgut in Fließpapier vorgekeimt. Nach drei Tagen wurden drei einheitlich große Keimlinge pro Gefäß eingesetzt und nach 5 Tagen auf zwei Pflanzen pro Gefäß reduziert. Eine Woche und 3 Wochen nach der Aussaat wurden die Pflanzen mit jeweils $0,15 \mathrm{~g} \mathrm{~N}$ in Form von Ammoniumnitrat nachgedüngt. Die Pflanzen wurden in der Klimakammer 50 Tage kultiviert. Gegossen wurde auf $65 \%$ der maximalen Wasserkapazität. Die Wasserversorgung erfolgte täglich auf der Waage mit entmineralisierten 
Wasser. Die Kultivierung der Pflanzen fand unter kontrollierten Bedingungen in der Klimakammer statt. Die Temperatur lag zwischen $26^{\circ} \mathrm{C}$ am Tage und $21^{\circ} \mathrm{C}$ in der Nacht. Die Luftfeuchtigkeit war tagsüber $70 \%$ und in der Nacht $60 \%$. Die Gefäße wurden, unabhängig von der Variante, zufällig in der Klimakammer aufgestellt und innerhalb eine Woche dreimal umgesetzt, um der Randeffekt zu minimieren. Nach 50 Tagen wurden die Sprosse abgeschnitten und im Trockenschrank bei $105^{\circ}$ C getrocknet. Danach wurden die Trockenmasseerträge und die P- Konzentration in der Pflanzen bestimmt (Scheffer und Pajenkamp, 1952), so dass der P- Entzug berechnet werden konnte.

Tabelle 34: Versuchsaufbau und Design des Gefäßversuches in der Klimakammer

\begin{tabular}{|c|c|c|c|}
\hline \multicolumn{2}{|c|}{ Versuchsaufbau } & \multicolumn{2}{|l|}{$\begin{array}{l}2 \text { ägyptische Böden, } 5 \text { Versuchs- } \\
\text { glieder, } 4 \text { Wiederholungen }\end{array}$} \\
\hline Kulturpflanze & & \multicolumn{2}{|l|}{$\begin{array}{l}\text { Mais, Sorte BIRKO, } 2 \text { Pflanzen } \\
\text { Gefäß }^{-1}\end{array}$} \\
\hline P- Düngung & & \multicolumn{2}{|l|}{$\begin{array}{l}\text { Alle P- Varianten erhielten } 31 \mathrm{mg} \mathrm{P} \\
\text { Gefäß }^{-1}\end{array}$} \\
\hline \multirow[t]{5}{*}{ P- Varianten } & (1) & Kontrolle, Versuchsboden ohne P & P 0 \\
\hline & (2) & Klärschlamm- Dollbergen & $5,87 \mathrm{~g} \mathrm{KS}$ \\
\hline & (3) & Klärschlamm- Göttingen & $4,54 \mathrm{~g} \mathrm{KS}$ \\
\hline & (4) & Klärschlamm- Osterode & $1,71 \mathrm{~g} \mathrm{KS}$ \\
\hline & (5) & Triplephosphat $20 \% \mathrm{P}$ & $155 \mathrm{mg}$ \\
\hline \multirow[t]{3}{*}{ Düngung } & & Stickstoff $\quad 450 \mathrm{mg} \mathrm{N}$ als $\mathrm{NH}_{4} \mathrm{NO}_{3}$ & $0,429 \mathrm{~g}$ Gefäß $^{-1}$ \\
\hline & & $150 \mathrm{mg} \mathrm{K}$ als $\mathrm{K}_{2} \mathrm{SO}_{4}$ & $0,334 \mathrm{~g} \mathrm{Gefäß}^{-1}$ \\
\hline & & $\begin{array}{l}\text { Magnesium } 30 \quad \mathrm{mg} \quad \mathrm{Mg} \text { als } \\
\text { MgSO } 4 \cdot 7 \mathrm{H} 2 \mathrm{O}\end{array}$ & $0,304 \mathrm{~g}_{\text {Gefäß }}{ }^{-1}$ \\
\hline Bewässerung & & $\begin{array}{l}\text { täglich auf ca. } 65 \quad \% \text { der max. } \\
\text { Wasserkapazität }\end{array}$ & \\
\hline Inkubationszeit & & 4 Wochen bei ca. $20^{\circ} \mathrm{C}$ vor der Saat & \\
\hline
\end{tabular}




\subsection{Ergebnisse}

Abbildung 24 gibt die Erträge und P- Entzüge des Maises auf dem lehmigen Tonboden Moschtohor wieder. Weder mit TSP noch mit den drei Klärschlämmen gab es signifikante Mehrerträge. Aber die P-Aufnahme aus TSP war auf $154 \%$ angestiegen. Ein signifikanter Anstieg des P- Entzuges bei den KS gab es nur bei dem KS von Osterode (20\%).
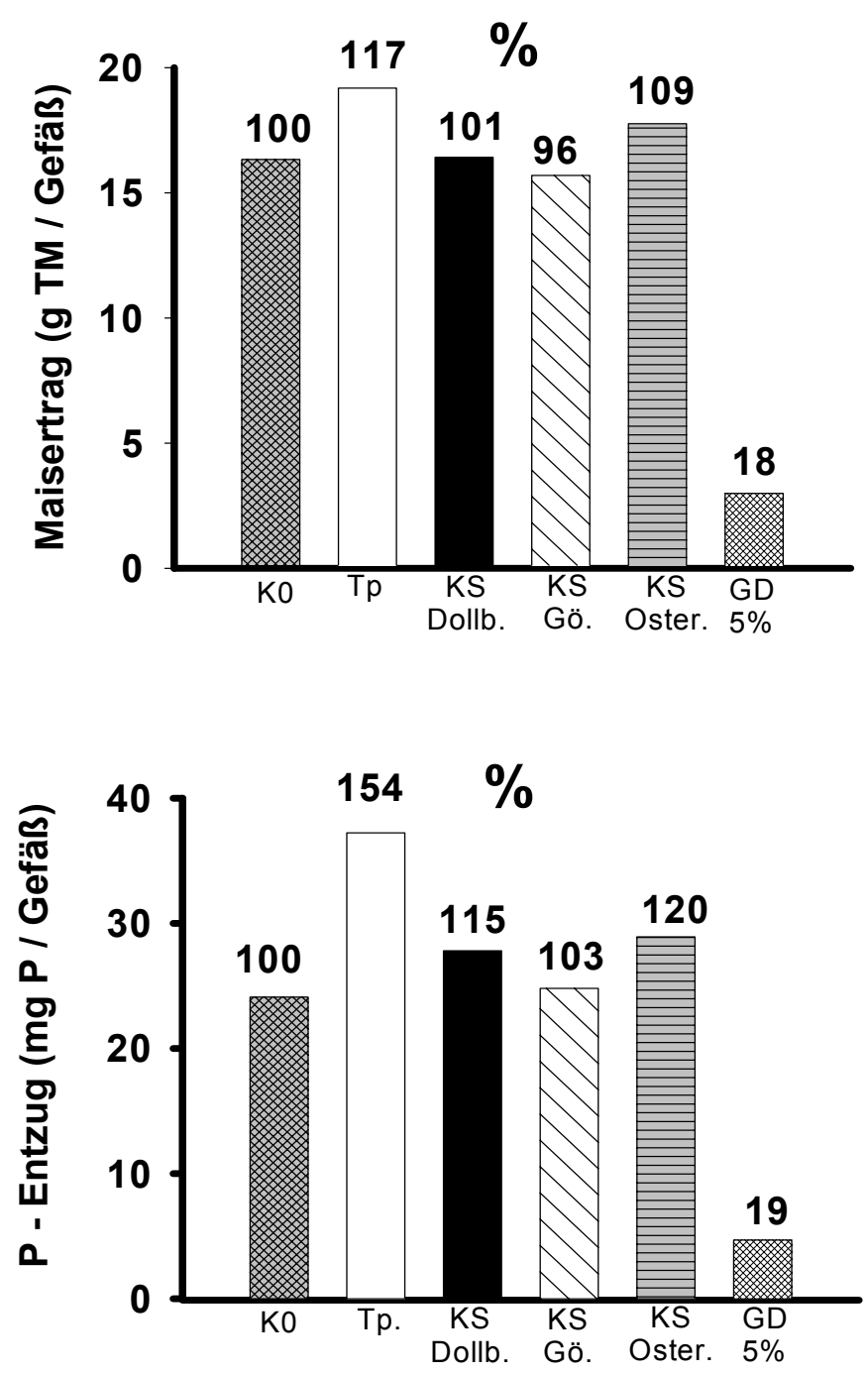

Abbildung 24: Abhängigkeit des Maisertrages und des $P$ - Entzuges von der P - Applikation zum Lehmboden Moschtohor $(K O=$ Kontrolle ohne $P, T p=T S P)$ 
Abbildung 25 gibt die Erträge und P- Entzüge des Maises auf dem tonigen Sandboden Meetkenana wieder. Das absolute Ertragsniveau lag deutlich niedriger als auf dem Boden Moschtohor. Auch hier gab es durch Düngung in keinem Fall signifikante Mehrerträge. Die statistische Analyse der P- Entzüge zeigt ein überraschendes Ergebnis, denn die P- Aufnahme aus der Variante KS- Osterode (152 \%) war höher als die aus TSP (129 \%).
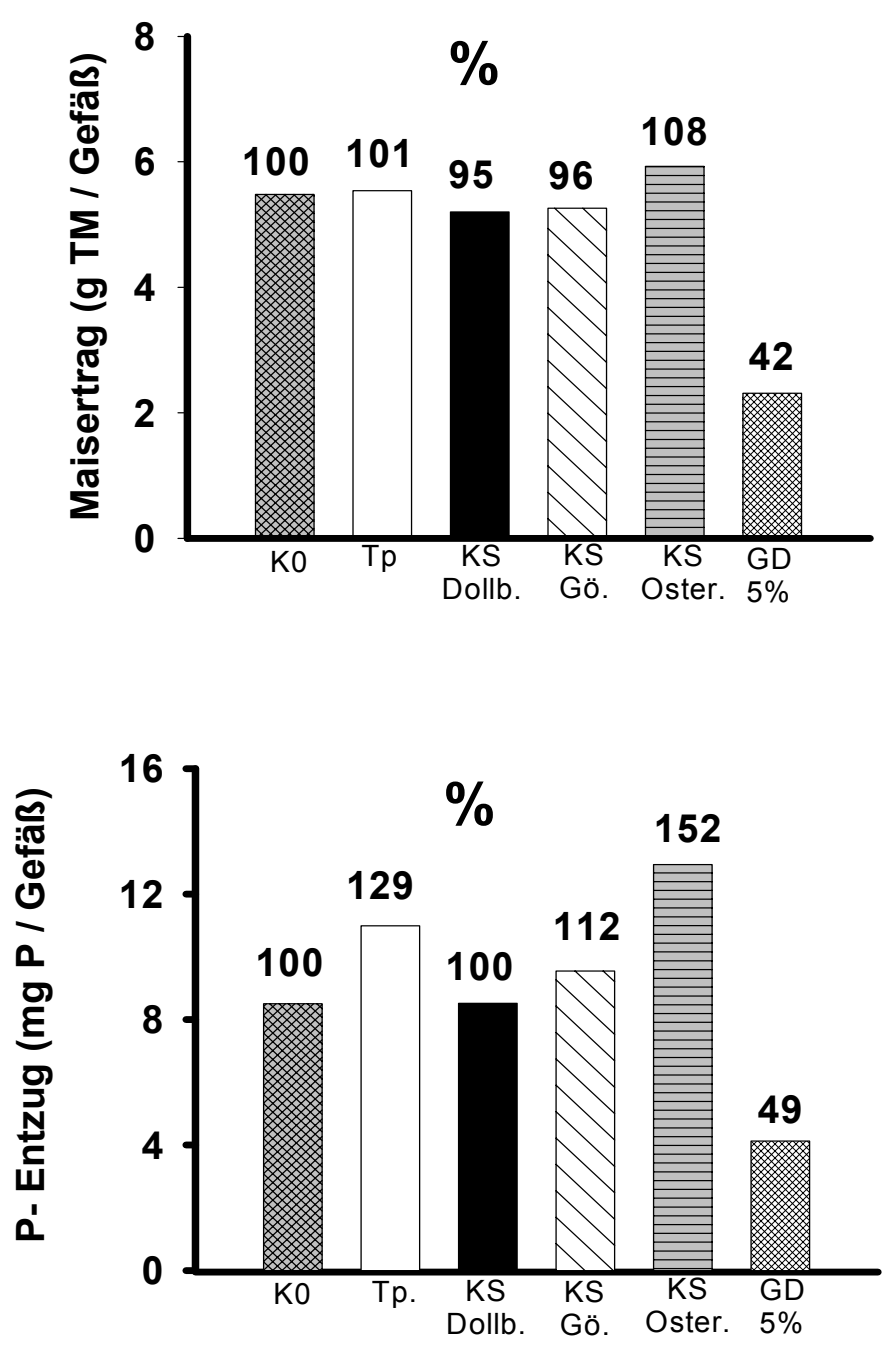

Abbildung 25: Abhängigkeit des Maisertrages und des $P$ - Entzuges von der $P$ - Applikation zum Sandboden Meetkenana $(K O=$ Kontrolle ohne $P, T p=T S P$ ) 
Das wesentlichste Resultat aus beiden Versuchen ist, dass die Maispflanzen von allen drei Klärschlämmen nur aus dem KS Osterode stets die höchsten PMengen aufnahmen. Die P- Aufnahmen aus den Klärschlämmen Dollbergen und Göttingen blieben stets unter der Signifikanzschwelle. Damit bestätigen diese Experimente die Einordnung der $3 \mathrm{KS}$ wie sie auch für den Sandboden Düshorn getroffen wurden. KS Dollbergen und Göttingen vertreten dort die Nr. 1 und 2 und KS Osterode die Nr. 10. Die KS Nr.1 und 2 verursachten auf dem Sandboden die geringsten P- Entzüge der $13 \mathrm{KS}$ (Tabelle 25) während sich der P- Entzug des KS Nr.10 (Osterode) mit 84\% nicht von P- Entzug der TSPVariante unterschied (Tabelle 26). 


\section{$5 \quad$ Abschließende Bemerkungen}

Versucht man alle Resultate im Zusammenhang zu betrachten, so ergibt sich, dass die P- Eliminierung aus dem Abwasser durch Eisensalze und besonders die Schlammkonditionierung mit Fe-Salzen zwar die P- Konzentration der geklärten Abwässer drastisch reduziert (Umweltbundsamt, 1997) und damit der Eutrophierung der Gewässer entgegenwirkt, aber den Landwirten, die den Klärschlamm verwerten wollen, ein Problem bereitet. Es konnte in beiden Teilen (Teil I, II) der Arbeit nachgewiesen werden, dass Klärschlämme mit einem Fe : P- Verhältnis, das stark zu Gunsten des Fe verschoben ist und damit eine geringe P-Sättigung aufweist, wichtige Parameter der P- Verfügbarkeit im Boden (P-Sorption, Orthophosphatkonzentration in der Bodenlösung) negativ beeinflusst.

Solche Veränderungen sind für den Pflanzenbau als ungünstig zu beurteilen. Deshalb sind solche KS als Sekundärrohstoffdünger abzulehnen. Der Landwirt hat einen Anspruch auf KS mit hoher P- Verfügbarkeit. Da es Verfahren zur PEliminierung ohne solche Eisensalze gibt (Klaus und Schönborn, 1999), ist seitens des Bodenschutzes daraufhin zu wirken, dass Klärschlämme erzeugt werden, die sowohl das Phosphat der Abwässer eliminieren (Vermeidung der Gewässereutrophierung) als auch das Phosphat in gut aufnehmbar Form enthalten. Denn die P- Vorräte der Welt sind begrenzt und das P- Recycling mit den KS kann helfen, die P-Vorräte zu schonen. Dass dies möglich ist, wurde mit einigen eisenarmen $\mathrm{KS}$ sehr gut demonstriert. 
In deutschen Kläranlagen werden 500000 t Eisensalze zur P- Eliminierung eingesetzt. Von den insgesamt 2,4 Mill. t TM Klärschlamm (KS) werden 38\% in der Landwirtschaft eingesetzt. Damit dürften beträchtliche Eisenmengen in Ackerböden gelangen. Da Eisenhydroxide /- oxide eine hohe Affinität zum Phosphat besitzen, bleibt zweifelhaft, ob das Phosphat derartiger EisenPhosphat- Verbindungen gut für Pflanzen verfügbar ist. Ferner ist unklar, ob derartige Eisenverbindungen im Boden vorkommendes Phosphat, z.B. aus der Bodenlösung adsorbieren, also die P- Verfügbarkeit im Boden senken, was nicht erwünscht wäre. Aus Sicht der Pflanzenernährung und des Phosphatrecyclings erschien es deshalb sinnvoll, diesen Problemen experimentell nachzugehen. Die Untersuchungen (Gefäßversuch mit Mitscherlichgefäßen) gliedern sich in drei Teile: I, II und III.

\section{Teil I}

\section{Ziel und Vorgehensweise}

Dieser Teil hatte zwei Ziele:

1. Zeitliche Verfolgung der Änderung der P-Sorption und der Konzentration an Orthophosphat $\left(\mathrm{P}_{\mathrm{i}}\right)$ in der Bodenlösung von Sand- und Schluffböden mit unterschiedlichem P- Gehalt, wenn zwei KS unterschiedlichen Eisengehaltes in die Böden eingebracht werden.

2. Ermittlung des Biomasseertrages und P-Aufnahme von Kulturpflanzen, die auf solch gedüngten Böden wachsen.

$\mathrm{CaHPO}_{4}$ diente als P- Mineraldünger zum Vergleich.

\section{Lösungsweg:}

In diesen Versuchen kamen stets zwei Klärschlämme mit folgenden Parametern zur Anwendung: 
KS Göttingen KS Salzhausen

(KS Gö)

(KS Sh)

$\mathrm{kg} \mathrm{t}^{-1} \mathrm{TM}$

P-Gehalt

Fe-Gehalt

Ca-Gehalt

$\mathrm{Fe}: \mathrm{P}$

mol Fe : mol P
12

65

197

$1: 0,18$

$1: 0,3$
25

39

21

$1: 0,64$

$1: 1,2$

Die P-Düngung basierte auf der Applikation von 5 t TM ha ${ }^{-1}$ als KS Gö mit $60 \mathrm{~kg}$ $P$ ha ${ }^{-1}$ bzw. $30 \mathrm{mg} \mathrm{P} \mathrm{kg}^{-1}$ Boden im Gefäßversuch. Gleiche P-Mengen wurden als KS Sh bzw. als $\mathrm{CaHPO}_{4}$ (stellvertretend für P-Mineraldünger) zugegeben.

\section{(A) Gefäßversuche zur Verfolgung der P- Verfügbarkeit im Boden}

Die Inkubation der Böden mit KS bzw. die Düngung mit $\mathrm{CaHPO}_{4}$ erfolgte in 8 niedersächsischen Lössböden (Parabraunerden) bzw. Sandböden und Quarzsand, z.T. in gestaffelten Mengen in einer Klimakammer für 12 Monate. In definierten Abständen (1 Monat, 6 bzw. 12 Monate) erfolgte die Bestimmung der P-Sorption, der Orthophosphatkonzentration in den Bodenlösungen, der pH-Werte und der Gehalte an oxalatlöslichem (amorphen) Eisen. Nach 12 Monaten erfolgte in dem auf kleinere Gefäße verteilten Boden ein Anbau von Weidelgras in der Drahthalle des Gewächshauses und eine 3-malige Ernte des Grases und Bestimmung seiner P-Aufnahme.

\section{Ergebnisse}

Das P-Sorptionsvermögen blieb in allen Böden nach Applikation von KS Sh (Fe $: P=1: 0,64)$ annähernd auf gleichem Niveau wie im Boden ohne $K S$, aber nach KS Gö (Fe : $P=1: 0,18)$ stieg es im Mittel um $16 \%(0-59 \%)$ an. Die $\mathrm{P}_{\mathrm{i}^{-}}$ Konzentration stieg bei KS Sh-Gabe im Mittel der Lehmböden um 34 \%, die der Sandböden um $15 \%$ an, sank aber nach KS Gö-Gabe um 13 bzw. 36 \% im 
Vergleich zur Kontrolle ab. Mit steigenden Applikationen von KS Gö (5, 10, 15 t $\mathrm{TM} \mathrm{ha}^{-1}$ ) stiegen nach 1 Monat die Konzentration des Bodens an oxalatlöslichem Fe und dazu die P-Sorption linear an $\left(r^{2}=0,6\right.$ bis 0,97$)$. Das Weidelgras nahm nach KS Gö Anwendung weniger $P$ auf als nach KS ShDüngung und viel weniger als aus $\mathrm{CaHPO}_{4}$ auf. Die geringe $\mathrm{P}$ - Aufnahme der Pflanzen nach KS Gö- Düngung korrespondierte mit der in den Böden verringerten $\mathrm{P}$ - Verfügbarkeit (erhöhte $\mathrm{P}$ - Sorption, verringerte $\mathrm{P}_{\mathrm{i}}$ Konzentration). KS mit einem $\mathrm{Fe}$ : P-Verhältnis von 1:0,18 kann dem Pflanzenbau nicht als Sekundärrohstoffdünger empfohlen werden.

\section{(B) Gefäßversuch zur Prüfung der P- Aufnahme von drei Kulturpflanzen}

Das Ziel war, Wachstum (Trockenmassebildung) und P- Aufnahme von den drei aufeinander folgenden Kulturpflanzen Mais, Rotklee und Weidelgras in Abhängigkeit von der P- Düngung mit den zwei eisenhaltigen Klärschlämmen (KS) im Vergleich zum "P- Mineraldünger" $\mathrm{CaHPO}_{4}$ in den Jahren 1999, 2000 und 2001 zu prüfen. Dazu wurden die zwei KS bzw. CaHPO $430 \mathrm{mg} \mathrm{P} / \mathrm{kg}$ Boden) in 4 Ackerböden (2 Schluffböden mit pH 6,3 bzw. 7,2; 2 Sandböden mit pH 4,3 bzw. 4,9), in Löß (C - Horizont einer Parabraunerde) und in Quarzsand (Mitscherlich - Gefäße) eingebracht. Die Böden bzw. Substrate besaßen unterschiedliche Ausgangsgehalte an Lactat-P. Die Versuchsglieder wurden vierfach wiederholt.

\section{Ergebnisse}

Im Vergleich zu CaHPO ${ }_{4}$ konnten die Kulturpflanzen nach Anwendung von KS Sh auf allen Ackerböden die gleichen P-Mengen aufnehmen. Der KS Gö wirkte anders: Aus den schluffreichen Ackerböden nahm Mais nur 64-82 \% und Rotklee 77-82 \% P im Vergleich zur $\mathrm{CaHPO}_{4}$-Variante auf. Dieses aus Sicht der P-Ausnutzbarkeit ungünstige Resultat für den KS Gö ist auf eine erhöhte PSorption im Boden und eine verringerte Orthophosphatkonzentration in der Bodenlösung zurückzuführen. Auf den sauren Sandböden hingegen erreichte der Mais 96-106 \% und der Rotklee 83-87 \% der P-Aufnahme im Vergleich zur $\mathrm{CaHPO}_{4}$ - Anwendung. Diese relativ günstige Wirkung des KS Gö (kalkkonditioniert) auf die P-Aufnahme und die TM-Bildung des Maises auf den 
Sandböden resultierte sowohl aus der Anhebung des Boden-pH von 4,3 auf 4,9 bzw. 4,9 auf 5,6 als auch aus der P-Aufnahme.

Insgesamt ergab sich aus den Gefäßversuchen mit den Kulturpflanzen, dass sie insbesondere auf den schweren Böden bei KS Gö- Düngung deutlich in ihrer $\mathrm{P}$ - Aufnahme hinter der aus $\mathrm{CaHPO}_{4}$ - und KS Sh- Düngung zurückblieben. Das steht in Übereinstimmung mit den Veränderungen der Kenngrößen der PVerfügbarkeit nach KS Gö- Anwendung.

\section{Teil II}

\section{Ziel und Vorgehensweise}

In Teil I war deutlich geworden, dass ein KS mit weitem Fe : P- Verhältnis $(1: 0,18)$ wie KS Gö nicht als direkter Ersatz für den P- Mineraldünger $\mathrm{CaHPO}_{4}$ geeignet ist. Es ergab sich die Frage, bis zu welchem Fe : P-Verhältnis der KS$P$ genau die gleiche $P$ - Düngewirkung wie der Mineraldünger hat. Ein solches Fe : P- Verhältnis wäre tolerierbar. Zu diesem Zweck wurden aus 13 Klärwerken (12 Niedersachsen, $1 \mathrm{NRW}$ ) KS mit weit gestaffelten molaren $\mathrm{Fe}$ : P- Verhältnissen von $1: 0,24$ bis $1: 7,94$ beschafft, gefriergetrocknet und im Vergleich zu Tripelsuperphosphat $\left(\mathrm{Ca}\left(\mathrm{H}_{2} \mathrm{PO}_{4}\right)_{2}\right)$ in einen mäßig mit $\mathrm{P}$ versorgten Sandboden (4,5 mg DL- P / 100g), $\left(\mathrm{pH}\left(\mathrm{CaCl}_{2}\right)\right.$ : 5,5) mit $30 \mathrm{mg} \mathrm{P} \mathrm{/}$ $\mathrm{kg}$ Boden eingebracht. Als Versuchspflanze diente Weidelgras (Drahthalle des Gewächshaus). Die P- Entzüge wurden mit mehreren Bodenkenngrößen korreliert

\section{Ergebnisse}

TSP steigerte die Sprossmasse auf $139 \%$, den P-Entzug auf $149 \%$. Bezogen auf die Kontrolle bewirkten die KS Sprosserträge von 98 bis $143 \%$, im Mittel $123 \%$, P-Entzüge von 107 bis $149 \%$, im Mittel $136 \%$.

Der P-Entzug der Pflanzen stieg in den Varianten mit KS linear mit der Orthophosphat-(Pi)-Konzentration der Bodenlösung an $\left(r^{2}=0,67\right)$ und sank ab: 
1. mit steigender P-Sorption $\left(r^{2}=0,33\right)$, 2. mit steigendem Gehalt an oxalatlöslichem Fe im Boden $\left(r^{2}=0,44\right)$ und 3. mit weiter werdendem Fe:PVerhältnis der KS $\left(r^{2}=0,86\right)$.

Die Nutzbarkeit des $\mathrm{P}$ aus den KS sinkt signifikant unter die von TSP ab, wenn Klärschlamm pro $1 \mathrm{~mol} \mathrm{Fe}(56 \mathrm{~g})$ weniger als $1,3 \mathrm{~mol} P(40 \mathrm{~g})$ enthält. Bei Sandböden sind die Kalkgehalte der KS für die Bodenreaktion unbedingt zu berücksichtigen.

\section{Teil III}

\section{Ziel und Vorgehensweise}

In Erweiterung der Problematik auf nicht deutsche Böden, sollte die PDüngewirkung von KS in zwei ägyptischen Böden mit Mais in einem Kurzzeitversuch getestet werden.

Drei ausgewählte KS mit deutlich variierten Fe : P- Verhältnissen (1:7,4; 1:5,4; $1: 1,4)$ wurden in zwei ägyptische Böden (lehmiger Tonboden und toniger Sandboden mit pH - Werten über 7,7) eingebracht. Die Kleingefäße mit $1 \mathrm{~kg}$ Boden standen in der Klimakammer. Zum Vergleich diente Triplesuperphosphat (TSP). Die P-Gabe betrug $31 \mathrm{mg} \mathrm{P} \mathrm{kg}^{-1}$ Boden. Die Pflanzen wuchsen 50 Tage und wurden danach auf TM und P- Entzug analysiert.

\section{Ergebnisse}

Die TM- Erträge des Maises auf dem sehr P- reichen lehmigen Tonboden wurden wie erwartet durch die P- Zugabe nicht beeinflusst. Die P- Aufnahme aus TSP war aber auf $154 \%$ im Vergleich zur Kontrolle (P 0) angestiegen. Ein signifikanter Anstieg des P- Entzuges bei den drei KS gab es nur mit dem KS Osterode (Fe : P-Verhältnissen 1:1,4), aber die P-Entzüge aus allen drei KS blieben signifikant unter denen aus TSP.

Die TM- Erträge des Maises auf dem tonigen Sandboden wurden ebenfalls durch die P- Zugabe nicht beeinflusst; denn nach VDLUFA- Vorgaben lag der 
Boden an der Grenze von Gehaltsklasse C/D. Die statistische Analyse der PEntzüge zeigt ein überraschendes Ergebnis, denn die P-Aufnahme aus der Variante KS Osterode mit dem Fe : P- Verhältnissen 1:1,4 war höher (152\%) als die aus TSP (129\%).

Im Prinzip bestätigte sich aber auch an den ägyptischen Böden, dass die PAufnahme aus KS mit molaren Fe : P- Verhältnissen größer als 0,79 (Tab. 32) gegenüber TSP deutlich geringer wird.

\section{Schlussfolgerungen:}

Aus Sicht der Pflanzenernährung dürfte ein wiederholtes Ausbringen von $\mathrm{KS}$ mit weitem $\mathrm{Fe}$ : P-Verhältnis die P-Dynamik im Boden in unerwünschter Richtung (zunehmende P-Sorption, Senkung der Orthophosphatkonzentration in der Bodenlösung) beeinflussen- ein Phänomen, das längerfristig verfolgt werden muss, denn eine Verringerung der P-Verfügbarkeit im Boden durch $\mathrm{KS}$ ist unerwünscht.

Aus Rücksicht auf die mögliche Erhöhung der P-Sorption und damit Reduzierung der Phosphatverfügbarkeit in den Böden wäre eine PEliminierung im Abwasser ohne Eisensalze als Fällungsmittel zu empfehlen. Auf alle Fälle sollte bei der Schlammkonditionierung auf eine weitere Eisensalzzugabe verzichtet werden.

$>\quad$ Damit die Landwirtschaftskammern eine bessere Beurteilung der PVerfügbarkeit der Klärschlämme vornehmen können, sollten in Zukunft die Fe-Gehalte generell mitanalysiert werden. Das ist bisher nicht der Fall. So könnten die Fe : P-Verhältnisse stets errechnet und Hinweise für die P-Verfügbarkeit gegeben werden.

Enthalten Klärschlämme je Mol Fe (56 g) weniger als 1,3 Mol P (40 g), so unterscheiden sie sich in Bezug auf die P- Aufnahme der Pflanzen auf einem Sandboden signifikant negativ von der TriplesuperphosphatWirkung. Solche Klärschlämme sollten nicht auf Ackerböden gelangen.

$>$ Insbesondere auf Sandböden sind die Kalkmengen in den Klärschlämmen zu berücksichtigen, da es bei einer Überkalkung solcher Böden zu Manganmangel kommen kann (eigene Beobachtungen). 


\section{$7 \quad$ Literaturverzeichnis}

Abwassertechnische Vereinigung (ATV, Hrsg.), 1996: Landwirtschaftliche Klärschlammverwertung. ATV-Information, 1-30.

Adams, F. (1974): Soil solution. In: The plant root and its environment. E.W. Carson (ed.), University Press of Virginia, Charlottesville, 441-481.

Bannick, C. und Hahn, J., (2001): Klärschlammverwertung aus Sicht des Bodenschutzes unter Berücksichtigung der Harmonisierung von Schadstoffeinträgen. Umweltbundesamt Berlin. ATV-DVWK-Schriftenreihe 27, Klärschlamm aktuell 1, 38-57.

Bannick, C.G. (2001): Grundsätze und Maßnahmen für eine vorsorgeorientierte Begrenzung von Schadstoffeinträgen in landwirtschaftlich genutzte Böden. Zeitschrift: Bodenschutz 3, 101-103.

Bannick, C.G., Bergs, C.G.\& Lindner, K. -H. (2002): Verwertung von Bioabfällen und Klärschlämmen. Einführung- Verordnung- VollzugshilfenAbfallwirtschaft in Forschung und Praxis, Bd. 123. Erich Schmidt Verlag. ISBN 3-503-06093-6.

Baran, E. (1985): Zusammensetzung und Düngewirkung von PhosphatFällungsprodukten aus der dritten Abwasserreinigungsstufe. Dissertation, Fachbereich Agrarwissenschaften, Universität Göttingen.

Beißner, L. (1997): Mobilisierung von Phosphor aus organischen und anorganischen P-Verbindungen durch Zuckerrübenwurzeln. Cuvillier Verlag Göttingen.

Bergs, C.G. (1998): Rechtliche Regelungen für die Verwertung von Klärschlämmen und sonstigen Sekundärrohstoffdüngern. Vortrag zur 9. Klärschlammberatertagung der LK Hannover am 1.12.1998 in Nienburg.

Egner, H., Riehm, H., u. Domingo, W.R. (1960): Untersuchungen über die chemische Bodenanalyse als Grundlage für die Beurteilung des 
Nährstoffzustandes der Böden. Kungl. Lantbrukshögskolans Annalen 26: 199215

Fritsch, F. und Werner, W. (1989): Einfluss langjähriger Klärschlammdüngung auf Löslichkeitskriterien der Bodenphosphate. Landwirtschaftliche Forschung 41, 305-311.

Frossard, E., Sinaj, S., Zhang, L.-M. and Morel, J.L. (1996): The fate of phosphorus in soil - plant systems. Soil Sci. Soc. Am. J. 60, 1248-1253.

Gerke, J. (1992): Phosphate, aluminium and iron in the soil solution of three different soils in relation to varying concentrations of citric acid. Z. Pflanzenern. Bodenk. 155, 339-343.

Gerke, J. (1995): Chemische Prozesse der Nährstoffmobilisierung in der Rhizosphäre und ihre Bedeutung für den Übergang vom Boden in die Pflanze. Cuvillier Verlag, Göttingen.

Gunreben, M. (2000): Landwirtschaftliche Klärschlammverwertung in Niedersachsen (Hrsg. Nieders. Landesamt für Ökologie). 1 - 164

Hahn, J. (2000): Ausstieg aus der "landwirtschaftlichen Klärschlammverwertung" - eine notwendige Harmonisierung im vorsorgenden Umweltschutz. Bodenschutz 3, 72-73.

Hahn, J. (2001): Vorsorgeorientierte Begrenzung von Schadstoffeinträgen in Böden am Beispiel der Klärschlammverwertung. Zeitschrift: Wasser, Luft und Boden 9, 60-67.

Keller, H. (2000): Einfluss wurzelbürtiger organischer Säuren auf das Cu-, Znund Cd-Aneignungsvermögen von Spinatgenotypen. Dissertation, Fachbereich Chemie, Universität Kaiserslautern.

Klaus, I. und Schönborn, Ch. (1999): Phosphatelimination - Mechanismen und Einflussfaktoren. Wasserwirtschaft Wassertechnik, 4; 22-26. 
Murphy, J., Riley, J. (1962): A modified single solution method for the determination of phosphate in natural waters. Anal. Chim. Acta, 27, 31-36.

Parfitt, R.L. (1978): Anionenadsorption. Adv. Agronomy, 30, 1-50.

Römer, W. und Gerke, J. (1998): Phosphat- und Eisenmobilisierung durch Wurzelexsudate bei Lupinus albus L. ;In: Lupinen in Forschung und Praxis (Hrsg. M. Wink), 1, 15 - 25

Römer, W. und Schilling, G. (1986): Phosphorus requirements of the wheat plant in various stages of its life cycle. Plant and Soil 91, 221-229.

Schaaf, H. und Janßen, E. (2000): Schwermetallgehalte von Wirtschafts- und Sekundärrohstoffdüngern und Schwermetallfrachten bei Anwendung nach den anerkannten Regeln der guten fachlichen Praxis. 112. VDLUFA-Kongress, Stuttgart-Hohenheim, im Druck.

Scheffer, F. und Pajenkamp, H. (1952): Phosphatbestimmung in Pflanzenaschen nach der Molybdän-Vanadin-Methode. Z. Pflanzenernähr. Bodenk. 56, 2-8.

Scheffer, F. und Schachtschabel, P. (2002): Lehrbuch der Bodenkunde, 15. Auflage, Spektrum Akademischer Verlag, Heidelberg. Berlin.

Scheffer, W. (2000): Abwasserbehandlung in der Kläranlage, Fällungs- und Flockungsmittel zur chemischen P-Elimination. Tagung der VDLUFAFachgruppen I, II und X in Dresden, 15.3.2000

Schilling, G. (2000): Pflanzenernährung und Düngung, Verlag Eugen Ulmer, Stuttgart.

Schüller, H. (1969): Die CAL- Methode, eine neue Methode zur Bestimmung des pflanzenverfügbaren Phosphates in Böden. Z. Pflanzenernährung und Bodenkunde 123, 48-63

Schwertmann und Amann (1998): zitiert in Scheffer und Schachtschabel: Lehrbuch der Bodenkunde, 14. Auflage, Enke-Verlag, Stuttgart, S. 265. 
Schwertmann, U. (1964): Differenzierung der Eisenoxide des Bodens durch Extraktion mit Ammonium-Oxalatlösung. Z. Pflanzenernähr. Bodenk., 105, 194202.

Schwertmann, U. (1998): in Scheffer und Schachtschabel: Lehrbuch der Bodenkunde, 14. Auflage, Enke-Verlag, Stuttgart, Kapitel 2, 6.2.5.

Suntheim, L. und Dittrich, B. (1996): Klärschlamm - (k)ein wertvoller P-Dünger. VDLUFA-Schriftenreihe 44, Kongressband 1996, 313-316.

Umweltbundesamt (Hrsg.) (1997): Daten zur Umwelt, Erich Schmidt-Verlag, Berlin.

Wachtel, H. (1976): Die Phosphatvorkommen und die Versorgung der Landwirtschaft. Z. Die Bodenkultur. 27, $318-325$

Weber, E. (1967): Grundriß der biologischen Statistik. 6. Auflage. Verlag Gustav- Fischer Stuttgart.

Werner, W., (1976): Untersuchungen zur Phosphatwirkung von Klärschlämmen aus der chemischen Abwasserreinigung. Landwirtschaftliche Forschung, Sonderheft 32/l, 177-185.

Zorn, W., Krause, O. und Kießling, G. (2001): Wirkung unterschiedlicher Abwasserbehandlungsverfahren auf die P-Verfügbarkeit von Klärschlamm im Gefäßversuch. 113. VDLUFA - Kongress am 20.9.2001 in Berlin (im Druck). 
Tabelle 8.1: EU-Grenzwertvorschläge für organische Schadstoffe im Klärschlamm bei landwirtschaftlicher Verwertung ${ }^{\text {a) }}$

\begin{tabular}{|c|c|}
\hline Schadstoff & $\begin{array}{c}\text { Grenzwertvorschlag } \\
\text { (mg/kg TS) }\end{array}$ \\
\hline $\begin{array}{l}\text { AOX } \\
\text { LAS } \\
\text { DEHP } \\
\text { Nonylphenol }{ }^{11} \\
\text { PAK (9) } \\
\text { PCB }(7)^{2)} \\
\text { PCDD/-F (TE) }\end{array}$ & $\begin{array}{c}500 \\
2600 \\
100 \\
50 \\
6 \\
0,8 \\
100 \mathrm{ng} \\
\end{array}$ \\
\hline
\end{tabular}

a) Stand: Arbeitspapier der EU vom 27.04.2000

1) $90 \%$ der Schlämme, die landwirtschaftlich verwertet werden, sollen mittelbzw. langfristig die „Zielwerte“ unterschreiten.

In Klammern: Auf den Phosphorgehalt bezogene Grenzwerte

2) Summe der PCB-Kongenere 28, 52, 101, 118, 138, 153, 180

Tabelle 8.2: EU-Grenzwertvorschläge für Schwermetalle im Klärschlamm zur landwirtschaftlichen Verwertung a) (Grenzwerte können alternativ auf Trockenrückstand oder auf Phosphorgehalt bezogen werden)

\begin{tabular}{lcccc}
\hline Schwermetall & \multicolumn{2}{c}{ Grenzwerte } & \multicolumn{2}{c}{ Zielwerte $^{1)}$} \\
& \multicolumn{2}{c}{$(\mathrm{mg} / \mathrm{kg}$ TS) } & Mittelfristig & (mg TS) \\
& Richtlinie & & & \\
& $86 / 278 /$ EWG & Vorschlag & (ca. 2015) & (ca. 2025) \\
\hline Cadmium & $20-40$ & $10(250)$ & 5 & 2 \\
Kupfer & $1000-1750$ & $1000(25000)$ & 800 & 600 \\
Quecksilber & $16-25$ & $10(250)$ & 5 & 2 \\
Nickel & $300-400$ & $300(7500)$ & 200 & 100 \\
Blei & $750-1200$ & $750(18750)$ & 500 & 200 \\
Zink & $2500-4000$ & $2500(62500)$ & 2000 & 1500 \\
Chrom & --- & $1000(25000)$ & 800 & 600 \\
\hline
\end{tabular}

a) Stand: Arbeitspapier der EU vom 27.04.2000

1) $90 \%$ der Schlämme, die landwirtschaftlich verwertet werden, sollen mittelbzw. langfristig die "Zielwerte“ unterschreiten.

In Klammern: Auf den Phosphorgehalt bezogene Grenzwerte 
Tabelle 8.3: Analysendaten zu den zwei gefriergetrockneten, applizierten Klärschlämme

\begin{tabular}{|c|c|c|c|}
\hline \multicolumn{2}{|c|}{ 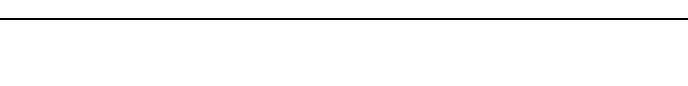 } & KS Göttingen (Gö) & KS Salzhausen (SH) \\
\hline \multicolumn{2}{|c|}{ TS } & $2,4 \%$ & $3,55 \%$ \\
\hline \multicolumn{2}{|c|}{$\mathrm{P}_{2} \mathrm{O}_{5}$} & $27,3 \mathrm{~kg} \mathrm{t}^{-1} \mathrm{TM}$ & $57,6 \mathrm{~kg} \mathrm{t}^{-1} \mathrm{TM}$ \\
\hline \multicolumn{2}{|c|}{$\mathrm{CaO}$} & & $30 \mathrm{~kg} \mathrm{t}^{-1} \mathrm{TM}$ \\
\hline \multicolumn{2}{|c|}{$\mathrm{pH}$} & & 6,98 (direkt) \\
\hline \multicolumn{2}{|c|}{$\mathrm{Fe}+$} & & $39158 \mathrm{mg} \mathrm{kg}^{-1} \mathrm{TM}$ \\
\hline \multicolumn{2}{|l|}{$\mathrm{Al}$} & & $12817 \mathrm{mg} \mathrm{kg}^{-1} \mathrm{TM}$ \\
\hline \multicolumn{2}{|c|}{ Org. Substanz } & & $70 \%$ in d. TM \\
\hline \multicolumn{2}{|c|}{ Gesamt- N } & & $33 \mathrm{~kg} \mathrm{t}^{-1} \mathrm{TM}$ \\
\hline \multicolumn{2}{|c|}{$\mathrm{NH}_{4}-\mathrm{N}$} & & $2 \mathrm{~kg} \mathrm{t}^{-1} \mathrm{TM}$ \\
\hline$>$ & $\mathrm{FeSO}_{4}$ zum aeroben Prozess & \multicolumn{2}{|c|}{$\mathrm{FeCl}_{3}$ zum aeroben Prozeß } \\
\hline$>$ & $\mathrm{FeCl}_{3}$ zum Dickschlamm & & \\
\hline$>$ & $\mathrm{Ca}(\mathrm{OH})_{2}$ zum Dickschlamm & & \\
\hline
\end{tabular}


Tabelle 8.4: P-Sorption von 5 Lehmböden (absolut und relativ) 1,6 und 12 Monate nach der P-Düngung

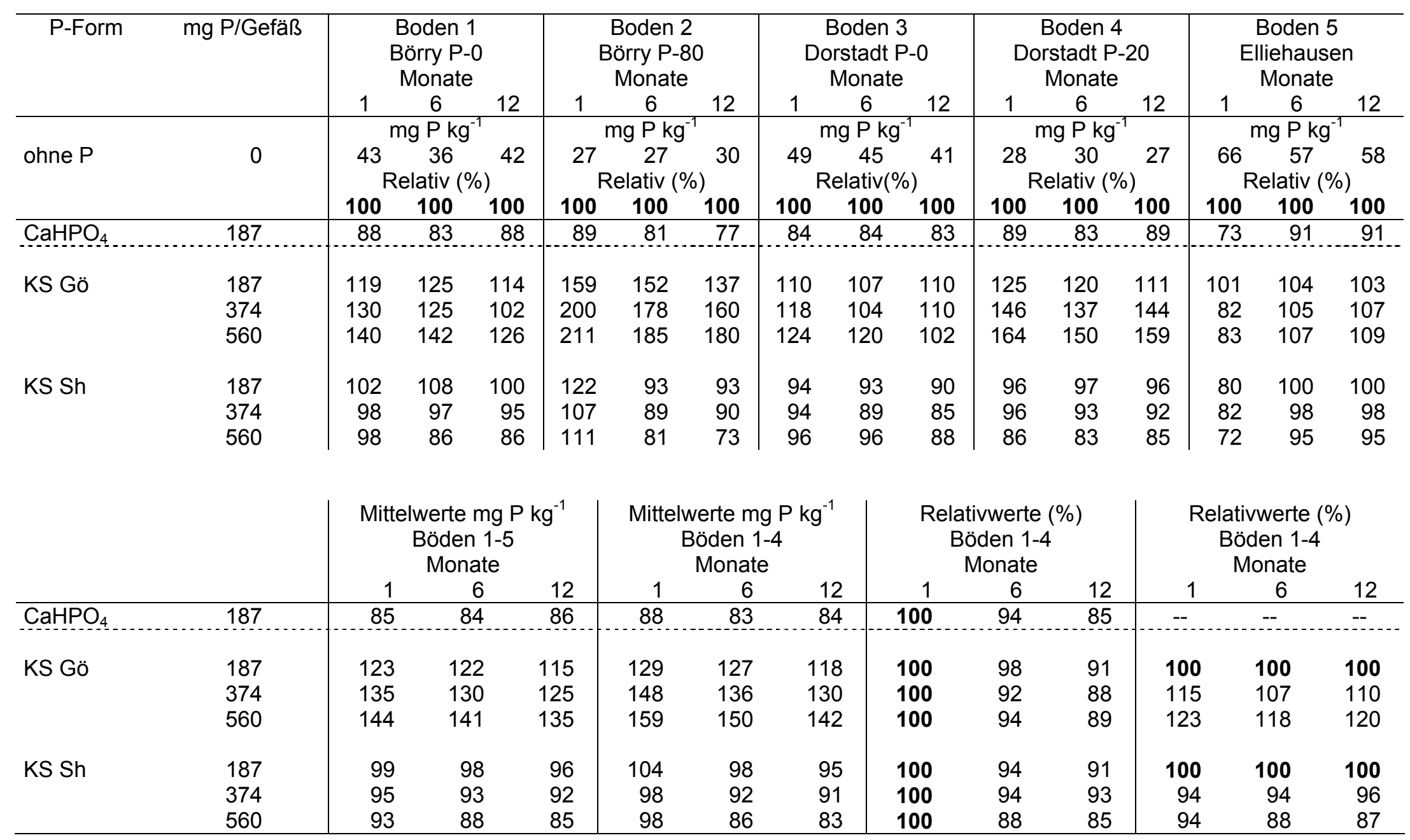


Tabelle 8.5: P-Sorption von 4 Sandböden (absolut und relativ) 1,6 und 12 Monate nach der P-Düngung

\begin{tabular}{|c|c|c|c|c|c|c|c|c|c|c|c|c|c|}
\hline \multirow[t]{2}{*}{ P-Form } & \multirow[t]{2}{*}{$\begin{array}{c}\mathrm{mg} \\
\mathrm{P} / \mathrm{Gefäß}\end{array}$} & \multicolumn{3}{|c|}{$\begin{array}{c}\text { Boden } 1 \\
\text { üshorn (Brache) } \\
\text { Monate }\end{array}$} & \multicolumn{3}{|c|}{$\begin{array}{c}\text { Boden } 2 \\
\text { Düshorn P-22 } \\
\text { Monate }\end{array}$} & \multicolumn{3}{|c|}{$\begin{array}{l}\text { Boden } 3 \\
\text { Rehburg } \\
\text { Monate }\end{array}$} & \multicolumn{3}{|c|}{$\begin{array}{c}\text { Boden } 4 \\
\text { Hodenhagen P-22 } \\
\text { Monate }\end{array}$} \\
\hline & & & 6 & 12 & 1 & 6 & 12 & 1 & 6 & 12 & 1 & 6 & 12 \\
\hline & & \multicolumn{3}{|c|}{$\mathrm{mg} \mathrm{P} \mathrm{kg}^{-1}$} & \multicolumn{3}{|c|}{$\mathrm{mg} \mathrm{P} \mathrm{kg}^{-1}$} & \multicolumn{3}{|c|}{$\mathrm{mg} \mathrm{P} \mathrm{kg}^{-1}$} & \multicolumn{3}{|c|}{$\mathrm{mg} \mathrm{P} \mathrm{kg}^{-1}$} \\
\hline ohne $\mathrm{P}$ & 0 & 72 & 83 & 77 & 27 & -- & 33 & 82 & 88 & 85 & 53 & 61 & 56 \\
\hline \multirow[t]{2}{*}{ ohne P } & \multirow[t]{2}{*}{0} & \multicolumn{3}{|c|}{ Relativ (\%) } & \multicolumn{3}{|c|}{ Relativ (\%) } & \multicolumn{3}{|c|}{ Relativ (\%) } & \multicolumn{3}{|c|}{ Relativ (\%) } \\
\hline & & 100 & 100 & 100 & 100 & -- & 100 & 100 & 100 & 100 & 100 & 100 & 100 \\
\hline $\mathrm{CaHPO}_{4}$ & 187 & 92 & 94 & 91 & 85 & $\therefore-$ & 82 & 95 & 91 & 97 & 82 & 89 & 95 \\
\hline \multirow{3}{*}{ KS Gö } & 187 & 108 & 99 & 103 & 140 & -- & 115 & 102 & 95 & 102 & 113 & 139 & 102 \\
\hline & 674 & 113 & 100 & 99 & 185 & -- & 127 & 105 & 95 & 102 & 126 & 118 & 109 \\
\hline & 560 & 119 & 101 & 105 & 222 & -- & 130 & 106 & 97 & 100 & 142 & 125 & 121 \\
\hline \multirow[t]{3}{*}{ KS Sh } & 187 & 102 & 99 & 97 & 115 & -- & 100 & 99 & 97 & 100 & 102 & 119 & 107 \\
\hline & 374 & 104 & 99 & 99 & 119 & -- & 94 & 100 & 100 & 100 & 102 & 100 & 100 \\
\hline & 560 & 106 & 100 & 97 & 126 & -- & 94 & 102 & 100 & 100 & 105 & 102 & 100 \\
\hline
\end{tabular}

\begin{tabular}{|c|c|c|c|c|c|c|c|c|c|c|}
\hline \multirow[b]{2}{*}{$\mathrm{CaHPO}_{4}$} & \multirow[b]{2}{*}{187} & \multicolumn{3}{|c|}{$\begin{array}{c}\text { Mittelwerte }\left(\mathrm{mg} \mathrm{P} \mathrm{kg}^{-1}\right) \\
\text { Böden 1-4 }\end{array}$} & \multicolumn{3}{|c|}{$\begin{array}{l}\text { Relativwerte (\%) } \\
\text { Böden 1-4 }\end{array}$} & \multicolumn{3}{|c|}{$\begin{array}{c}\text { Relativwerte (\%) } \\
\text { Böden 1-4 }\end{array}$} \\
\hline & & 91 & 90 & 91 & 100 & 100 & 100 & -- & - & -- \\
\hline \multirow[t]{3}{*}{ KS Gö } & 187 & 112 & 107 & 106 & 100 & 96 & 91 & 100 & 100 & 100 \\
\hline & 674 & 132 & 104 & 109 & 100 & 79 & 83 & 114 & 94 & 103 \\
\hline & 560 & 147 & 107 & 114 & 100 & 73 & 78 & 127 & 96 & 108 \\
\hline \multirow[t]{3}{*}{ KS Sh } & 187 & 102 & 105 & 101 & 100 & 102 & 97 & 100 & 100 & 100 \\
\hline & 374 & 106 & 100 & 98 & 100 & 94 & 92 & 102 & 95 & 97 \\
\hline & 560 & 110 & 101 & 98 & 100 & 92 & 89 & 106 & 96 & 97 \\
\hline
\end{tabular}


Tabelle8.6: $P_{i-}$ Konzentration $\left(\mathrm{mg} \mathrm{L}^{-1}\right)$ in der Bodenlösung 1 Monat, 6 und 12 Monate nach der P-Applikation zu den Lehmböden

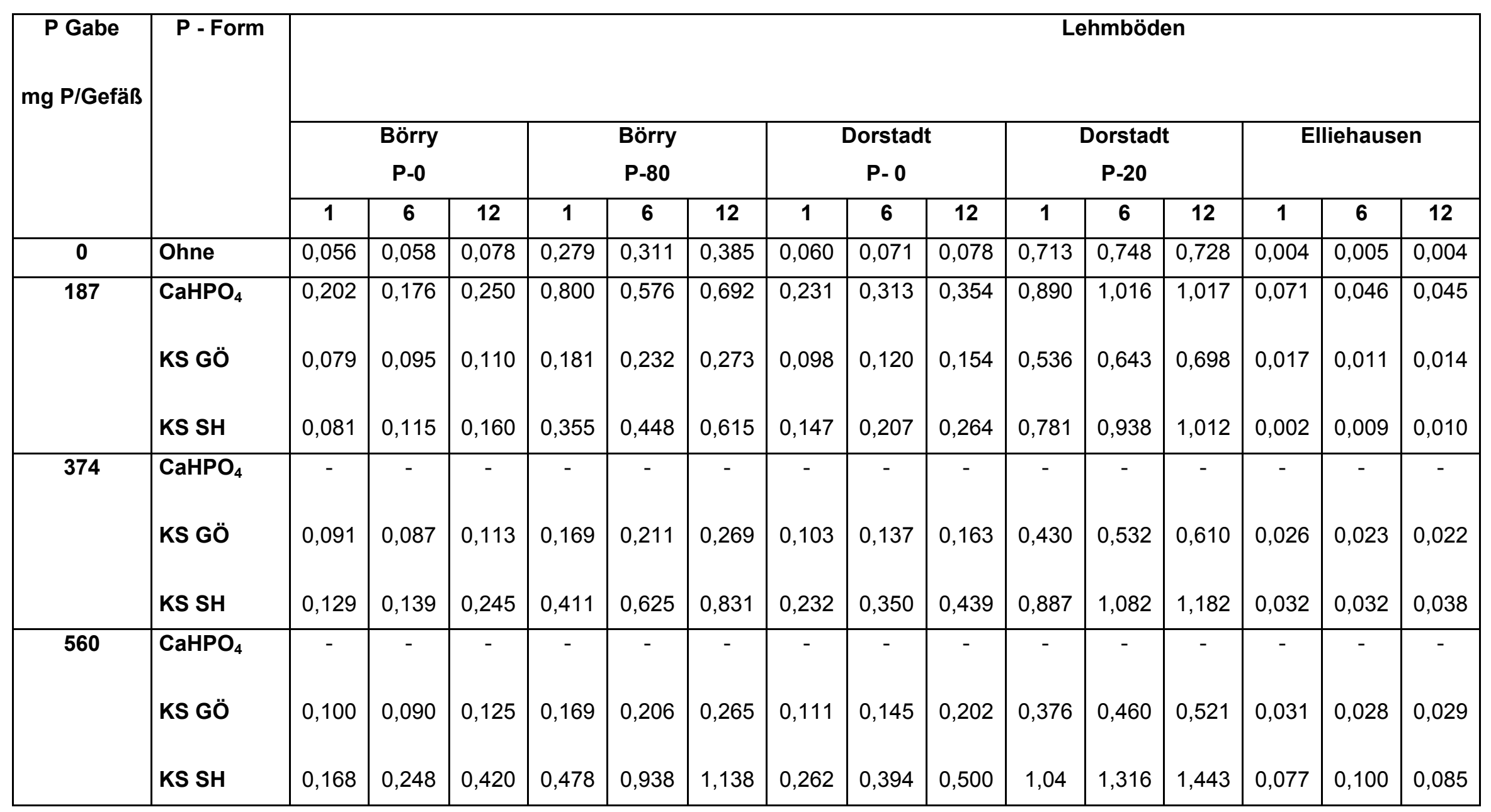


Tabelle 8.7: $P_{i-}$ Konzentration $\left(\mathrm{mg} \mathrm{L}^{-1}\right)$ in der Bodenlösung 1 Monat, 6 und 12 Monate nach der P-Applikation zu den Sandböden

\begin{tabular}{|c|c|c|c|c|c|c|c|c|c|c|c|c|c|}
\hline \multirow{3}{*}{$\begin{array}{c}\text { P Gabe } \\
\text { mg P Gefäß }{ }^{-1}\end{array}$} & \multirow[t]{3}{*}{ P - Form } & \multicolumn{12}{|c|}{ Sandböden } \\
\hline & & \multicolumn{3}{|c|}{$\begin{array}{l}\text { Düshorn } \\
\text { (Brache) }\end{array}$} & \multicolumn{3}{|c|}{$\begin{array}{l}\text { Düshorn } \\
(P-22)\end{array}$} & \multicolumn{3}{|c|}{$\begin{array}{l}\text { Rehburg } \\
\text { Loccum }\end{array}$} & \multicolumn{3}{|c|}{$\begin{array}{c}\text { Hodenhagen } \\
(P-22)\end{array}$} \\
\hline & & 1 & 6 & 12 & 1 & 6 & 12 & 1 & 6 & 12 & 1 & 6 & 12 \\
\hline 0 & Ohne & 0,041 & 0,021 & 0,019 & 0,276 & 0,245 & 0,280 & 0,011 & 0,014 & 0,013 & 0,142 & 0,111 & 0,118 \\
\hline 187 & $\begin{array}{l}\mathrm{CaHPO}_{4} \\
\text { KS GÖ } \\
\text { KS SH }\end{array}$ & $\begin{array}{l}0,086 \\
0,048 \\
0,054\end{array}$ & $\begin{array}{l}0,043 \\
0,021 \\
0,043\end{array}$ & $\begin{array}{r}0,034 \\
0,019 \\
0,033\end{array}$ & $\begin{array}{l}0,677 \\
0,131 \\
0,293\end{array}$ & $\begin{array}{l}0,499 \\
0,147 \\
0,271\end{array}$ & $\begin{array}{l}0,477 \\
0,177 \\
0,299\end{array}$ & $\begin{array}{l}0,052 \\
0,020 \\
0,038\end{array}$ & $\begin{array}{l}0,032 \\
0,016 \\
0,025\end{array}$ & $\begin{array}{r}0,028 \\
0,010 \\
0,026\end{array}$ & $\begin{array}{r}0,283 \\
0,094 \\
0,148\end{array}$ & $\begin{array}{l}0,183 \\
0,077 \\
0,123\end{array}$ & $\begin{array}{r}0,164 \\
0,085 \\
0,121\end{array}$ \\
\hline 374 & $\begin{array}{l}\mathrm{CaHPO}_{4} \\
\text { KS GÖ } \\
\text { KS SH }\end{array}$ & $\begin{array}{c}- \\
0,049 \\
0,081\end{array}$ & $\begin{array}{c}- \\
0,061 \\
0,058\end{array}$ & $\begin{array}{c}- \\
0,019 \\
0,048\end{array}$ & $\begin{array}{c}- \\
0,191 \\
0,378\end{array}$ & $\begin{array}{c}- \\
0,105 \\
0,388\end{array}$ & $\begin{array}{c}- \\
0,116 \\
0,446\end{array}$ & $\begin{array}{c}- \\
0,025 \\
0,059\end{array}$ & $\begin{array}{c}- \\
0,019 \\
0,037\end{array}$ & $\begin{array}{c}- \\
0,012 \\
0,035\end{array}$ & $\begin{array}{c}- \\
0,089 \\
0,174\end{array}$ & $\begin{array}{c}- \\
0,057 \\
0,160\end{array}$ & $\begin{array}{c}- \\
0,064 \\
0,172\end{array}$ \\
\hline 560 & $\begin{array}{l}\mathrm{CaHPO}_{4} \\
\mathrm{KS} \mathrm{GÖ} \\
\mathrm{KS} \mathrm{SH}\end{array}$ & $\begin{array}{c}- \\
0,083 \\
0,111\end{array}$ & $\begin{array}{c}- \\
0,020 \\
0,073\end{array}$ & $\begin{array}{c}- \\
0,020 \\
0,064\end{array}$ & $\begin{array}{c}- \\
0,238 \\
0,418\end{array}$ & $\begin{array}{c}- \\
0,116 \\
0,541\end{array}$ & $\begin{array}{c}- \\
0,136 \\
0,624\end{array}$ & $\begin{array}{c}- \\
0,036 \\
0,051\end{array}$ & $\begin{array}{c}- \\
0,023 \\
0,044\end{array}$ & $\begin{array}{c}- \\
0,018 \\
0,057\end{array}$ & $\begin{array}{c}- \\
0,145 \\
0,211\end{array}$ & $\begin{array}{c}- \\
0,048 \\
0,0194\end{array}$ & $\begin{array}{c}- \\
0,062 \\
0,231\end{array}$ \\
\hline
\end{tabular}


Tabelle 8.8: pH-Werte der Lehmböden 1 Monat, 6 und 12 Monate nach der P-Applikation (CaCl 20,01 M)

\begin{tabular}{|c|c|c|c|c|c|c|c|c|c|c|c|c|c|c|c|c|}
\hline \multirow{3}{*}{$\begin{array}{c}\text { P Gabe } \\
\text { mg P/Gefäß }\end{array}$} & \multirow[t]{3}{*}{$P$ - Form } & \multicolumn{15}{|c|}{ Lehmböden } \\
\hline & & \multicolumn{3}{|c|}{$\begin{array}{l}\text { Börry } \\
\text { P-0 }\end{array}$} & \multicolumn{3}{|c|}{$\begin{array}{l}\text { Börry } \\
\text { P-80 }\end{array}$} & \multicolumn{3}{|c|}{$\begin{array}{c}\text { Dorstadt } \\
\text { P-0 }\end{array}$} & \multicolumn{3}{|c|}{$\begin{array}{c}\text { Dorstadt } \\
\text { P-20 }\end{array}$} & \multicolumn{3}{|c|}{ Elliehausen } \\
\hline & & 1 & 6 & 12 & 1 & 6 & 12 & 1 & 6 & 12 & 1 & 6 & 12 & 1 & 6 & 12 \\
\hline 0 & Ohne & 7,20 & 7,20 & 7,40 & 6,22 & 6,24 & 6,25 & 7,17 & 7,15 & 7,20 & 7,07 & 7,10 & 7,20 & 7,70 & 7,65 & 7,65 \\
\hline \multirow[t]{3}{*}{187} & $\mathrm{CaHPO}_{4}$ & 7,26 & 7,3 & 7,45 & 6,23 & 6,24 & 6,20 & 7,12 & 7,00 & 7,30 & 7,05 & 7,10 & 7,20 & 7,70 & 7,70 & 7,70 \\
\hline & KS GÖ & 7,64 & 7,55 & 7,65 & 7,29 & 7,15 & 7,15 & 7,53 & 7,40 & 7,50 & 7,38 & 7,30 & 7,45 & 7,9 & 7,80 & 7,80 \\
\hline & KS SH & 7,21 & 7,25 & 7,4 & 6,17 & 6,15 & 6,10 & 7,08 & 7,15 & 7,25 & 7,03 & 7,10 & 7,20 & 7,70 & 7,70 & 7,70 \\
\hline \multirow[t]{3}{*}{374} & $\mathrm{CaHPO}_{4}$ & - & - & - & - & - & - & - & - & - & - & - & - & - & - & - \\
\hline & KS GÖ & 7,69 & 7,6 & 7,75 & 7,47 & 7,37 & 7,55 & 7,59 & 7,45 & 7,60 & 7,47 & 7,40 & 7,50 & 7,90 & 7,85 & 7,80 \\
\hline & KS SH & 7,06 & 7,15 & 7,3 & 5,97 & 5,90 & 5,85 & 7,06 & 7,05 & 7,20 & 7,03 & 7,00 & 7,05 & 7,70 & 7,70 & 7,70 \\
\hline \multirow[t]{3}{*}{560} & $\mathrm{CaHPO}_{4}$ & - & - & - & - & - & - & - & - & - & - & - & - & - & - & - \\
\hline & KS GÖ & 7,73 & 6,65 & 7,8 & 7,53 & 7,47 & 7,60 & 7,62 & 7,50 & 7,65 & 7,51 & 7,45 & 7,60 & 7,88 & 7,85 & 7,80 \\
\hline & KS SH & 7,01 & 7,10 & 7,20 & 5,95 & 5,70 & 5,65 & 6,96 & 7,00 & 7,10 & 6,92 & 6,90 & 7,00 & 7,65 & 7,65 & 7,60 \\
\hline
\end{tabular}


Tabelle 8. 9: $\mathrm{pH}$ - Werte der Sandböden 1 Monat, 6 und 12 Monate nach der P-Applikation $\left(\mathrm{CaCl}_{2}\right.$ 0,01 M)

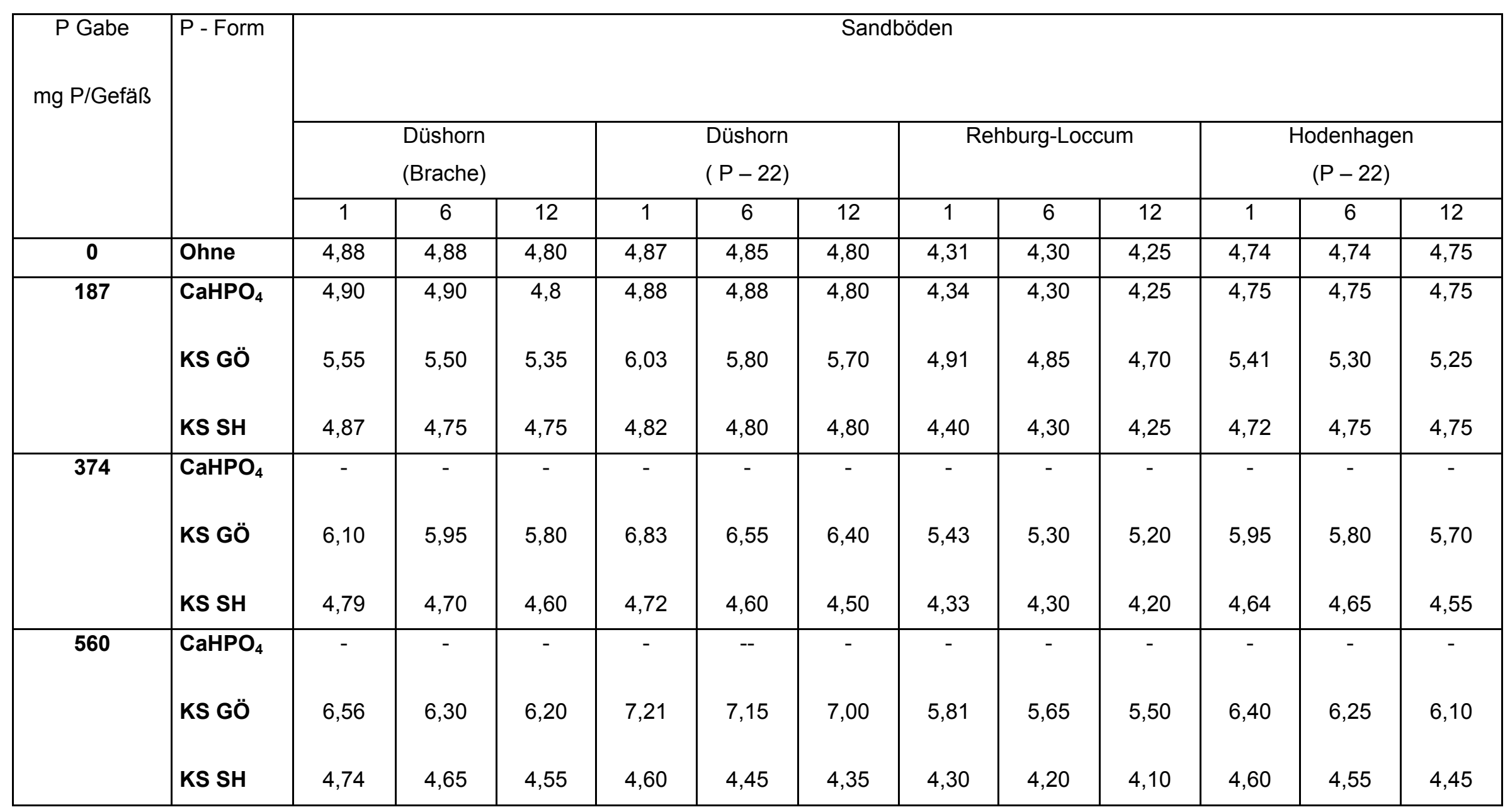


Tabelle 8.10: P - Gehalte der Lehmböden (CAL-Extraktion) 1 Monat, 6 und 12 Monate nach der P-Applikation (mg P 100g ${ }^{-1}$ )

\begin{tabular}{|c|c|c|c|c|c|c|c|c|c|c|c|c|c|c|c|c|}
\hline \multirow{3}{*}{$\begin{array}{c}\text { P Gabe } \\
\text { mg P/Gefäß }\end{array}$} & \multirow[t]{3}{*}{ P - Form } & \multicolumn{15}{|c|}{ Lehmböden } \\
\hline & & \multicolumn{3}{|c|}{$\begin{array}{l}\text { Börry } \\
\text { P- } 0\end{array}$} & \multicolumn{3}{|c|}{$\begin{array}{l}\text { Börry } \\
\text { P- } 80\end{array}$} & \multicolumn{3}{|c|}{$\begin{array}{c}\text { Dorstadt } \\
\text { P- } 0\end{array}$} & \multicolumn{3}{|c|}{$\begin{array}{c}\text { Dorstadt } \\
\text { P- } 20\end{array}$} & \multicolumn{3}{|c|}{ Elliehausen } \\
\hline & & 1 & 6 & 12 & 1 & 6 & 12 & 1 & 6 & 12 & 1 & 6 & 12 & 1 & 6 & 12 \\
\hline 0 & Ohne & 7,9 & 8,12 & 8 & 16,3 & 15,59 & 15,55 & 9,5 & 9,04 & 9,33 & 26,3 & 24,84 & 24,45 & 1,7 & 1,67 & 1,62 \\
\hline 187 & $\begin{array}{l}\mathrm{CaHPO}_{4} \\
\text { KS GÖ } \\
\text { KS SH }\end{array}$ & $\begin{array}{r}16,5 \\
11,2 \\
11,3\end{array}$ & $\begin{array}{r}11,00 \\
11,91 \\
11,62\end{array}$ & $\begin{array}{r}7,15 \\
12,27 \\
11,8\end{array}$ & $\begin{array}{l}20,2 \\
20,8 \\
20,0\end{array}$ & $\begin{array}{r}19,23 \\
19,78 \\
20,10\end{array}$ & $\begin{array}{r}18,5 \\
18,82 \\
20,15\end{array}$ & $\begin{array}{r}13,6 \\
12,8 \\
13,6\end{array}$ & $\begin{array}{l}13,32 \\
11,73 \\
12,57\end{array}$ & $\begin{array}{r}13,22 \\
10,64 \\
11,38\end{array}$ & $\begin{array}{r}32,1 \\
28,9 \\
31,4\end{array}$ & $\begin{array}{l}30,63 \\
26,63 \\
29,36\end{array}$ & $\begin{array}{c}29 \\
23,99 \\
27,3\end{array}$ & $\begin{array}{l}, 9 \\
3,6 \\
3,4\end{array}$ & $\begin{array}{l}3,4 \\
2,6 \\
2,51\end{array}$ & $\begin{array}{r}2,52 \\
1,9 \\
1,79\end{array}$ \\
\hline 374 & $\begin{array}{l}\mathrm{CaHPO}_{4} \\
\text { KS GÖ } \\
\text { KS SH }\end{array}$ & $\begin{array}{c}- \\
15,2 \\
13,8\end{array}$ & $\begin{array}{c}- \\
12,46 \\
15,15\end{array}$ & $\begin{array}{c}- \\
10,21 \\
17,3\end{array}$ & $\begin{array}{c}- \\
23,7 \\
24,3\end{array}$ & $\begin{array}{c}- \\
21,85 \\
23,01\end{array}$ & $\begin{array}{c}- \\
19,7 \\
21,92\end{array}$ & $\begin{array}{c}- \\
15,5 \\
18,3\end{array}$ & $\begin{array}{c}- \\
13,77 \\
17,59\end{array}$ & $\begin{array}{c}- \\
12,42 \\
16,54\end{array}$ & $\begin{array}{c}- \\
32,2 \\
33,2\end{array}$ & $\begin{array}{c}- \\
28,01 \\
32,89\end{array}$ & $\begin{array}{c}- \\
26,65 \\
31,9\end{array}$ & $\begin{array}{c}- \\
3,5 \\
6,2\end{array}$ & $\begin{array}{c}- \\
2,8 \\
4,85\end{array}$ & $\begin{array}{c}- \\
2,1 \\
3,52\end{array}$ \\
\hline 560 & $\begin{array}{l}\mathrm{CaHPO}_{4} \\
\text { KS GÖ } \\
\text { KS SH }\end{array}$ & $\begin{array}{c}- \\
19,3 \\
17,7\end{array}$ & $\begin{array}{c}- \\
14,06 \\
19,34\end{array}$ & $\begin{array}{c}- \\
9,8 \\
20,25\end{array}$ & $\begin{array}{c}- \\
26,1 \\
28,5\end{array}$ & $\begin{array}{c}- \\
23,82 \\
24,69\end{array}$ & $\begin{array}{c}- \\
21,5 \\
21,00\end{array}$ & $\begin{array}{c}- \\
18,1 \\
23,3\end{array}$ & $\begin{array}{c}- \\
16,10 \\
22,47\end{array}$ & $\begin{array}{c}- \\
13,73 \\
21,52\end{array}$ & $\begin{array}{c}- \\
35,3\end{array}$ & $\begin{array}{c}- \\
31,00 \\
37,04\end{array}$ & $\begin{array}{c}- \\
26,5 \\
33,75\end{array}$ & $\begin{array}{c}- \\
7,8\end{array}$ & $\begin{array}{c}- \\
7,49 \\
6,72\end{array}$ & $\begin{array}{c}- \\
6,75 \\
5,05\end{array}$ \\
\hline
\end{tabular}


Tabelle 8.11: P - Gehalte der Sandböden (DL-Extraktion) 1 Monat, 6 und 12 Monaten nach der P-Applikation (mg P 100g-1)

\begin{tabular}{|c|c|c|c|c|c|c|c|c|c|c|c|c|c|}
\hline \multirow{3}{*}{$\begin{array}{c}\text { P Gabe } \\
\mathrm{mg} \mathrm{P} / \text { Gefäß }\end{array}$} & \multirow[t]{3}{*}{ P - Form } & \multicolumn{12}{|c|}{ Sandböden } \\
\hline & & \multicolumn{3}{|c|}{$\begin{array}{l}\text { Düshorn } \\
\text { (Brache) }\end{array}$} & \multicolumn{3}{|c|}{$\begin{array}{l}\text { Düshorn } \\
(P-22)\end{array}$} & \multicolumn{3}{|c|}{ Rehburg-Loccum } & \multicolumn{3}{|c|}{$\begin{array}{l}\text { Hodenhagen } \\
\qquad(P-22)\end{array}$} \\
\hline & & 1 & 6 & 12 & 1 & 6 & 12 & 1 & 6 & 12 & 1 & 6 & 12 \\
\hline 0 & Ohne & 12,9 & 12,06 & 12,42 & 16,3 & 12,47 & 12,63 & 7,9 & 6,93 & 7,35 & 22,6 & 17,56 & 18,2 \\
\hline 187 & $\begin{array}{l}\mathrm{CaHPO}_{4} \\
\text { KS GÖ } \\
\text { KS SH }\end{array}$ & $\begin{array}{r}17,5 \\
14,63 \\
16,96\end{array}$ & $\begin{array}{r}13,50 \\
11,48 \\
14,40\end{array}$ & $\begin{array}{c}10,15 \\
9.0 \\
13,5\end{array}$ & $\begin{array}{l}22,6 \\
24,7 \\
17,7\end{array}$ & $\begin{array}{r}14,85 \\
14,63 \\
14,72\end{array}$ & $\begin{array}{r}12,63 \\
13,49 \\
12,6\end{array}$ & $\begin{array}{r}10,4 \\
10,1 \\
11,9\end{array}$ & $\begin{array}{l}10,13 \\
7,88 \\
9,90\end{array}$ & $\begin{array}{r}9,65 \\
5,95 \\
7,98\end{array}$ & $\begin{array}{l}26,6 \\
25,3 \\
25,3\end{array}$ & $\begin{array}{r}20,88 \\
18,09 \\
17,73\end{array}$ & $\begin{array}{r}15,65 \\
12,66 \\
12,55\end{array}$ \\
\hline 374 & $\begin{array}{l}\mathrm{CaHPO}_{4} \\
\text { KS GÖ } \\
\text { KS SH }\end{array}$ & $\begin{array}{c}- \\
19,1 \\
20,5\end{array}$ & $\begin{array}{c}- \\
13,68 \\
18,23\end{array}$ & $\begin{array}{c}- \\
9,6 \\
16,95\end{array}$ & $\begin{array}{c}- \\
27,9 \\
21,6\end{array}$ & $\begin{array}{c}- \\
21,15 \\
16,92\end{array}$ & $\begin{array}{c}- \\
22,09 \\
13,65\end{array}$ & $\begin{array}{c}- \\
11,3 \\
16,3\end{array}$ & $\begin{array}{c}- \\
8,64 \\
12,69\end{array}$ & $\begin{array}{l}6,42 \\
9,56\end{array}$ & $\begin{array}{c}- \\
27,7 \\
26,5\end{array}$ & $\begin{array}{c}- \\
20,43 \\
21,78\end{array}$ & $\begin{array}{c}- \\
14,78 \\
17,4\end{array}$ \\
\hline 560 & $\begin{array}{l}\mathrm{CaHPO}_{4} \\
\mathrm{KS} \mathrm{GÖ} \\
\text { KS SH }\end{array}$ & $\begin{array}{c}- \\
21,4 \\
22,8\end{array}$ & $\begin{array}{c}- \\
16,20 \\
19,26\end{array}$ & $\begin{array}{c}- \\
12,5 \\
16,49\end{array}$ & $\begin{array}{c}- \\
29,4 \\
28,6\end{array}$ & $\begin{array}{c}- \\
25,74 \\
18,81\end{array}$ & $\begin{array}{c}- \\
28,5 \\
15,2\end{array}$ & $\begin{array}{c}- \\
14,0 \\
19,9\end{array}$ & $\begin{array}{c}- \\
10,13 \\
15,30\end{array}$ & $\begin{array}{c}- \\
7,31 \\
12,4\end{array}$ & $\begin{array}{c}- \\
29,9 \\
29,9\end{array}$ & $\begin{array}{c}- \\
23,04 \\
22,86\end{array}$ & $\begin{array}{c}- \\
17,38 \\
17,74\end{array}$ \\
\hline
\end{tabular}


Tabelle 8.12: Relativerträge an Maistrockenmasse je Gefäß

\begin{tabular}{|c|c|c|c|c|c|c|c|c|c|c|c|c|}
\hline \multirow[t]{4}{*}{ Böden } & \multicolumn{12}{|c|}{ P- Düngungsvarianten (mg $\mathrm{P} \mathrm{kg}^{-1}$ Boden) } \\
\hline & \multicolumn{3}{|c|}{ Kontrolle } & \multicolumn{2}{|c|}{$\mathrm{CaHPO}_{4}$} & \multicolumn{3}{|c|}{ KS Gö } & \multicolumn{3}{|c|}{ KS Sh } & \multirow[t]{3}{*}{ GD 5\% } \\
\hline & 0 & & 44 & 87 & 187 & 44 & 87 & 187 & 44 & 87 & 187 & \\
\hline & g TM & rel. & rel. & rel. & rel. & rel. & rel. & rel. & rel. & rel. & rel. & \\
\hline Quarzsand & - & - & 100 & 100 & 100 & 30 & 28 & 26 & 15 & 25 & 37 & 18 \\
\hline Elliehausen & 29,9 & 100 & 217 & 300 & 471 & 137 & 186 & 276 & 171 & 250 & 450 & 45 \\
\hline Rehburg-Loccum & 90,5 & 100 & 134 & 149 & 157 & 125 & 152 & 171 & 112 & 148 & 149 & 60 \\
\hline Düshorn (Brache) & 116,6 & 100 & - & - & 117 & - & - & 138 & - & - & 130 & 15 \\
\hline Börry P- 0 & 149,6 & 100 & - & - & 141 & - & - & 121 & - & - & 122 & 13 \\
\hline Börry P- 80 & 231,3 & 100 & - & - & 110 & - & - & 112 & - & - & 107 & 15 \\
\hline
\end{tabular}


Tabelle 8.13: Relative P-Entzüge des Mais je Gefäß

\begin{tabular}{|c|c|c|c|c|c|c|c|c|c|c|c|c|}
\hline \multirow[t]{4}{*}{ Böden } & \multicolumn{12}{|c|}{ P- Düngungsvarianten (mg $\mathrm{P} \mathrm{kg}^{-1}$ Boden) } \\
\hline & \multicolumn{3}{|c|}{ Kontrolle } & \multicolumn{2}{|c|}{$\mathrm{CaHPO}_{4}$} & \multicolumn{3}{|c|}{ KS Gö } & \multicolumn{3}{|c|}{ KS Sh } & \multirow[t]{3}{*}{ GD 5\% } \\
\hline & 0 & & 44 & 87 & 187 & 44 & 87 & 187 & 44 & 87 & 187 & \\
\hline & g TM & rel. & rel. & rel. & rel. & rel. & rel. & rel. & rel. & rel. & rel. & \\
\hline Quarzsand & - & - & 100 & 100 & 100 & 30 & 32 & 25 & 20 & 36 & 37 & 36 \\
\hline Elliehausen & 21,6 & 100 & 181 & 220 & 375 & 140 & 167 & 239 & 160 & 230 & 380 & 48 \\
\hline Rehburg-Loccum & 100,5 & 100 & 114 & 107 & 119 & 110 & 121 & 131 & 111 & 111 & 131 & 27 \\
\hline Düshorn (Brache) & 116,5 & 100 & - & - & 114 & - & - & 111 & - & - & 123 & 22 \\
\hline Börry P- 0 & 105,1 & 100 & - & - & 163 & - & - & 133 & - & - & 168 & 21 \\
\hline Börry P- 80 & 240,8 & 100 & - & - & 127 & - & - & 99 & - & - & 111 & 18 \\
\hline
\end{tabular}


Tabelle 8.14: Analysendaten zu den 13 Klärschlämmen (Gehalte in der TM), nach LUFA-Hameln

\begin{tabular}{|c|c|c|c|c|c|c|c|c|c|c|c|c|c|c|}
\hline Parameter & & KS 1 & KS 2 & KS 3 & KS 4 & KS 5 & KS 6 & KS 7 & KS 8 & KS 9 & KS 10 & KS 11 & KS 12 & KS 13 \\
\hline Org. & $\%$ & 45,3 & 38,2 & 35,3 & 38,4 & 50,8 & 38,3 & 27,3 & 30,6 & 62 & 44,8 & 71,1 & 27,7 & 67,8 \\
\hline \multicolumn{15}{|l|}{ Substanz } \\
\hline $\mathrm{N}_{\text {ges. }}$ & $\mathrm{kg} / \mathrm{t}$ & 26,3 & 22,2 & 22,1 & 18,2 & 34,8 & 17,2 & 23,1 & 14,8 & 34,7 & 23,9 & 51,6 & 24 & 53,1 \\
\hline $\mathrm{P}_{2} \mathrm{O}_{5}$ & $\mathrm{~kg} / \mathrm{t}$ & 20,1 & 26 & 27,7 & 45 & 78,4 & 55,9 & 31,6 & 32 & 88,6 & 68,9 & 65,5 & 34,8 & 72,1 \\
\hline $\mathrm{K}_{2} \mathrm{O}$ & $\mathrm{kg} / \mathrm{t}$ & 1,12 & 1,95 & 2,41 & 1,49 & 1,90 & 2,66 & 2,12 & 3,46 & 2,27 & 1,75 & 10,3 & 3,39 & 16,5 \\
\hline $\mathrm{CaO}$ & $\mathrm{kg} / \mathrm{t}$ & 235 & 271 & 320 & 249 & 58,6 & 222 & 338 & 314 & 64,7 & 218 & 35,3 & 390 & 48,2 \\
\hline $\mathrm{MgO}$ & $\mathrm{kg} / \mathrm{t}$ & 3,59 & 6,78 & 8,44 & 9,87 & 5,11 & 9,28 & 4,44 & 10,4 & 6,2 & 8 & 6,31 & 6 & 10,7 \\
\hline Bas. wirk. & $\mathrm{kg} / \mathrm{t}$ & 195 & 242 & 284 & 207 & 51,5 & 201 & 319 & 272 & 34,1 & 180 & 19 & 343 & 24,8 \\
\hline \multicolumn{15}{|l|}{$\mathrm{CaO}$} \\
\hline $\mathrm{Fe}$ & $\mathrm{kg} / \mathrm{t}$ & 65,1 & 61,8 & 53,1 & 55,8 & 79 & 54,3 & 27,5 & 26,4 & 54,6 & 42,1 & 37,7 & 10,7 & 7,0 \\
\hline $\mathrm{Al}$ & $\mathrm{kg} / \mathrm{t}$ & 68,1 & 7,9 & 12,4 & 11,8 & 18,6 & 17,6 & 11,9 & 16,7 & 15,7 & 15,7 & 19 & 7,8 & 21,5 \\
\hline
\end{tabular}

KS = Klärschlämme, siehe Tab. 20 Seite 59 
Tabelle 8.15: Mengen an gefriergetrocknetem Klärschlamm, die bei einer Applikation von ca. $30 \mathrm{mg} P \mathrm{~kg}^{-1}$ Boden in die Gefäße eingearbeitet wurden sowie die je $\mathrm{kg}$ Boden eingesetzten Fe- und CaO- Mengen

\begin{tabular}{lcccc}
\hline Klärwerk /Boden & $\mathrm{Nr}$. & $\begin{array}{c}\mathrm{g} \mathrm{KS} / \mathrm{kg} \\
\text { Boden }\end{array}$ & $\begin{array}{c}\mathrm{mg} \mathrm{Fe} / \mathrm{kg} \\
\text { Boden }\end{array}$ & $\begin{array}{c}\mathrm{g} \mathrm{CaO} / \mathrm{kg} \\
\text { Boden }\end{array}$ \\
\hline Dollbergen & 1 & 3,4 & 221,2 & 0,80 \\
Göttingen & 2 & 2,64 & 163,2 & 0,72 \\
Gronau & 3 & 2,48 & 131,7 & 0,80 \\
Hameln & 4 & 1,52 & 84,8 & 0,38 \\
Einbeck & 5 & 0,87 & 68,8 & 0,05 \\
Herzberg & 6 & 1,22 & 66,3 & 0,27 \\
Gütersloh & 7 & 2,17 & 59,7 & 0,73 \\
Northeim & 8 & 2,13 & 56,3 & 0,67 \\
Uelzen & 9 & 0,78 & 42,6 & 0,05 \\
Osterode & 10 & 1,00 & 42,1 & 0,22 \\
Salzhausen & 11 & 1,05 & 39,6 & 0,04 \\
Ütze & 12 & 1,97 & 21,1 & 0,77 \\
Schöppenstedt & 13 & 0,95 & 6,7 & 0,05 \\
hohe Fe- Gabe & & & 735 & --- \\
niedrige Fe- Gabe & & & 235 & -- \\
hohe CaO- Gabe & & & --- & 484 \\
niedrige CaO- Gabe & & & --- & 161 \\
hohe Fe + hohe CaO- & & & 735 & 484 \\
Gabe & & & & \\
\hline
\end{tabular}


Tabelle 8.16: Absolute und relative Trockenmasseerträge und P-Entzüge von Mais in Abhängigkeit von der P-Quelle auf dem Lehmboden (Moschtohor)

\begin{tabular}{lcrcc}
\hline \multicolumn{1}{c}{ Variante } & $\begin{array}{c}\text { TM- Ertrag } \\
\text { g / Gefäß }\end{array}$ & $\begin{array}{r}\text { rel. } \\
\%\end{array}$ & $\begin{array}{c}\text { P- Entzug } \\
\text { mg / Gefäß }\end{array}$ & $\begin{array}{c}\text { rel. } \\
\%\end{array}$ \\
\hline K 0 & 16,34 & 100 & 24,12 & 100 \\
Triple-P & 19,19 & 117 & 37,25 & 154 \\
\hline KS- Dollbergen & 16,43 & 101 & 27,83 & 115 \\
KS- Göttingen & 15,71 & 96 & 24,81 & 103 \\
KS- Osterode & 17,78 & 109 & 28,95 & 120 \\
KS $\varnothing$ & $\mathbf{1 6 , 6 4}$ & $\mathbf{1 0 2}$ & $\mathbf{2 7 , 2 0}$ & $\mathbf{1 1 3}$ \\
\hline GD $_{5 \%}$ (Tukey) & 3,01 & 18,4 & 4,69 & 19,44 \\
\hline
\end{tabular}

Tabelle 8.17: Absolute und relative Trockenmasseerträge und P-Entzüge von Mais in Abhängigkeit von der P-Quelle auf dem Sandboden (Meetkenana)

\begin{tabular}{lcccc}
\hline \multicolumn{1}{c}{ Variante } & $\begin{array}{c}\text { TM- Ertrag } \\
\text { g / Gefäß }\end{array}$ & $\begin{array}{r}\text { rel. } \\
\%\end{array}$ & $\begin{array}{c}\text { P- Entzug } \\
\text { mg / Gefäß }\end{array}$ & $\begin{array}{c}\text { rel. } \\
\%\end{array}$ \\
\hline K 0 & 5,48 & 100 & 8,51 & 100 \\
Triple-P & 5,54 & 101 & 10,99 & 129 \\
\hline KS- Dollbergen & 5,20 & 95 & 8,52 & 100 \\
KS- Göttingen & 5,26 & 96 & 9,55 & 112 \\
KS- Osterode & 5,93 & 108 & 12,94 & 152 \\
KS $\varnothing$ & $\mathbf{5 , 4 6}$ & $\mathbf{1 0 0}$ & $\mathbf{1 0 , 3 4}$ & $\mathbf{1 2 1}$ \\
\hline GD $_{5 \%}$ (Tukey) & 2,31 & 42,15 & 4,13 & 48,53 \\
\hline
\end{tabular}



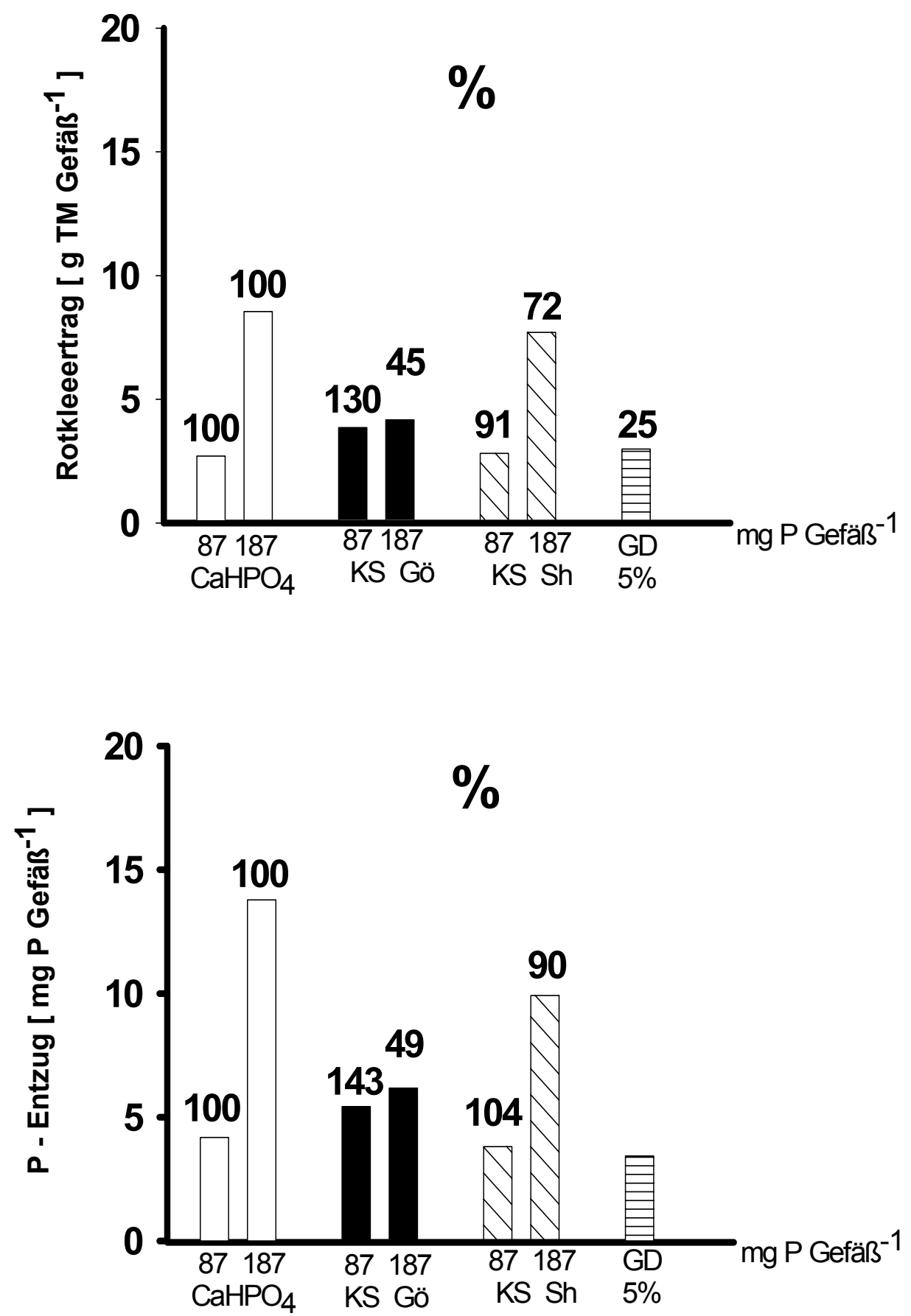

Abb.8.1: $\quad$ Abhängigkeit des Rotkleeertrages und des $P$ - Entzuges von der P - Applikation zum Quarzsand 

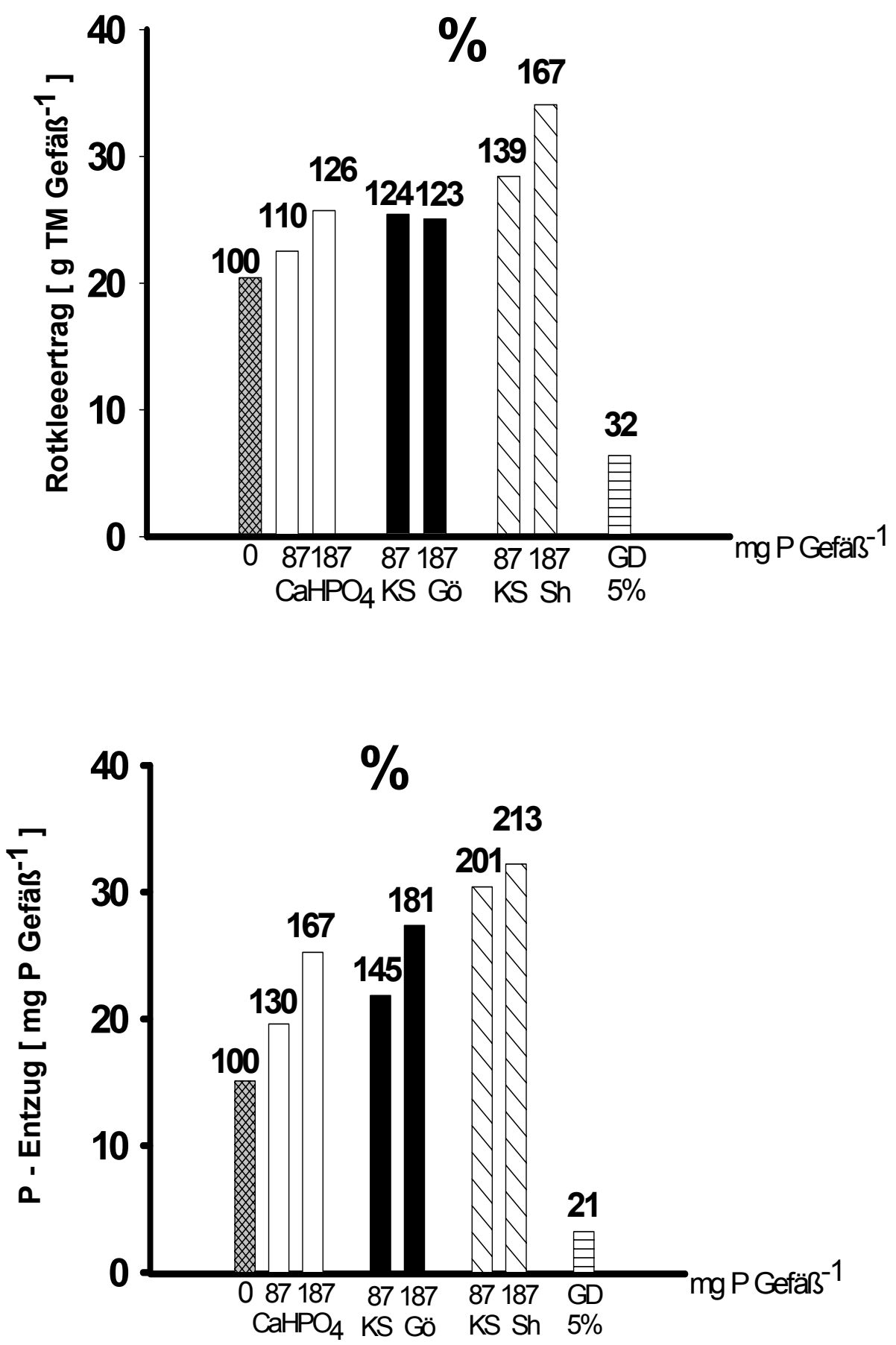

Abbildung 8.2: Abhängigkeit des Rotkleeertrages und des $P$ - Entzuges von der $P$ - Applikation zum Boden Eliehausen 

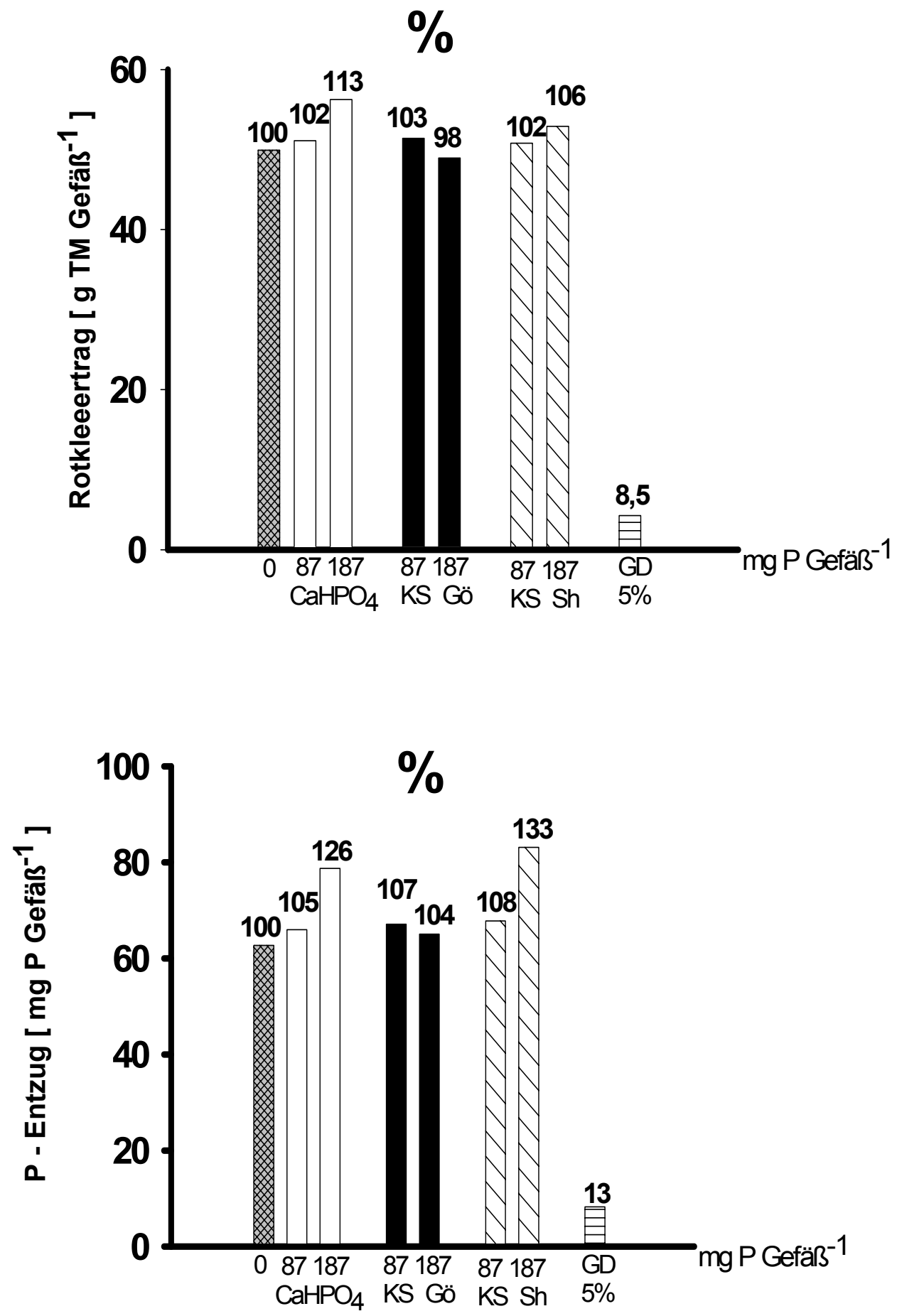

Abbildung 8.3: Abhängigkeit des Rotkleeertrages und des $P$ - Entzuges von der $P$ - Applikation zum Boden Rehburg - Loccum 

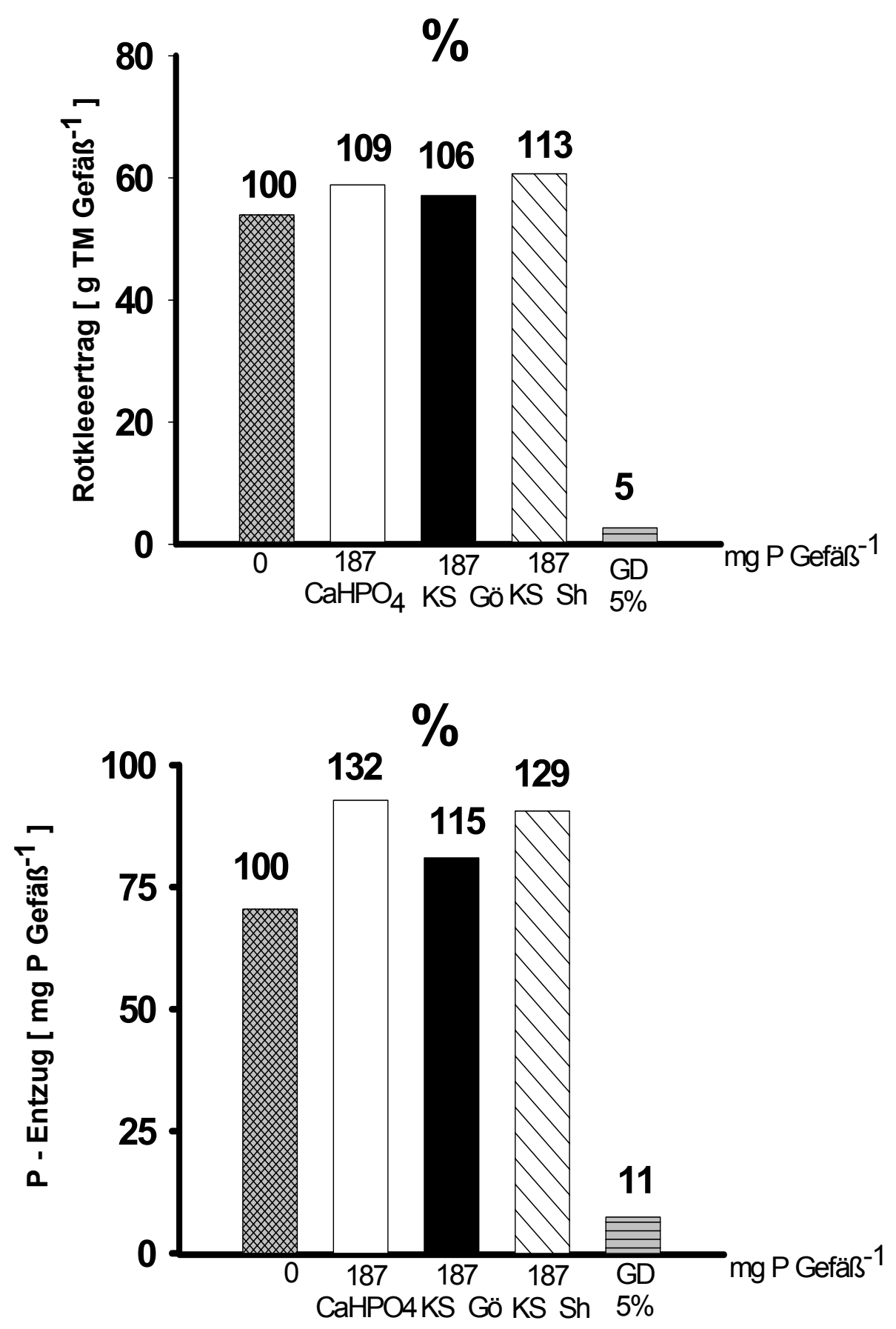

Abb.8.4: Abhängigkeit des Rotkleeertrages und des $\mathrm{P}$ - Entzuges von der P - Applikation zum Boden Düshorn (Brache) 

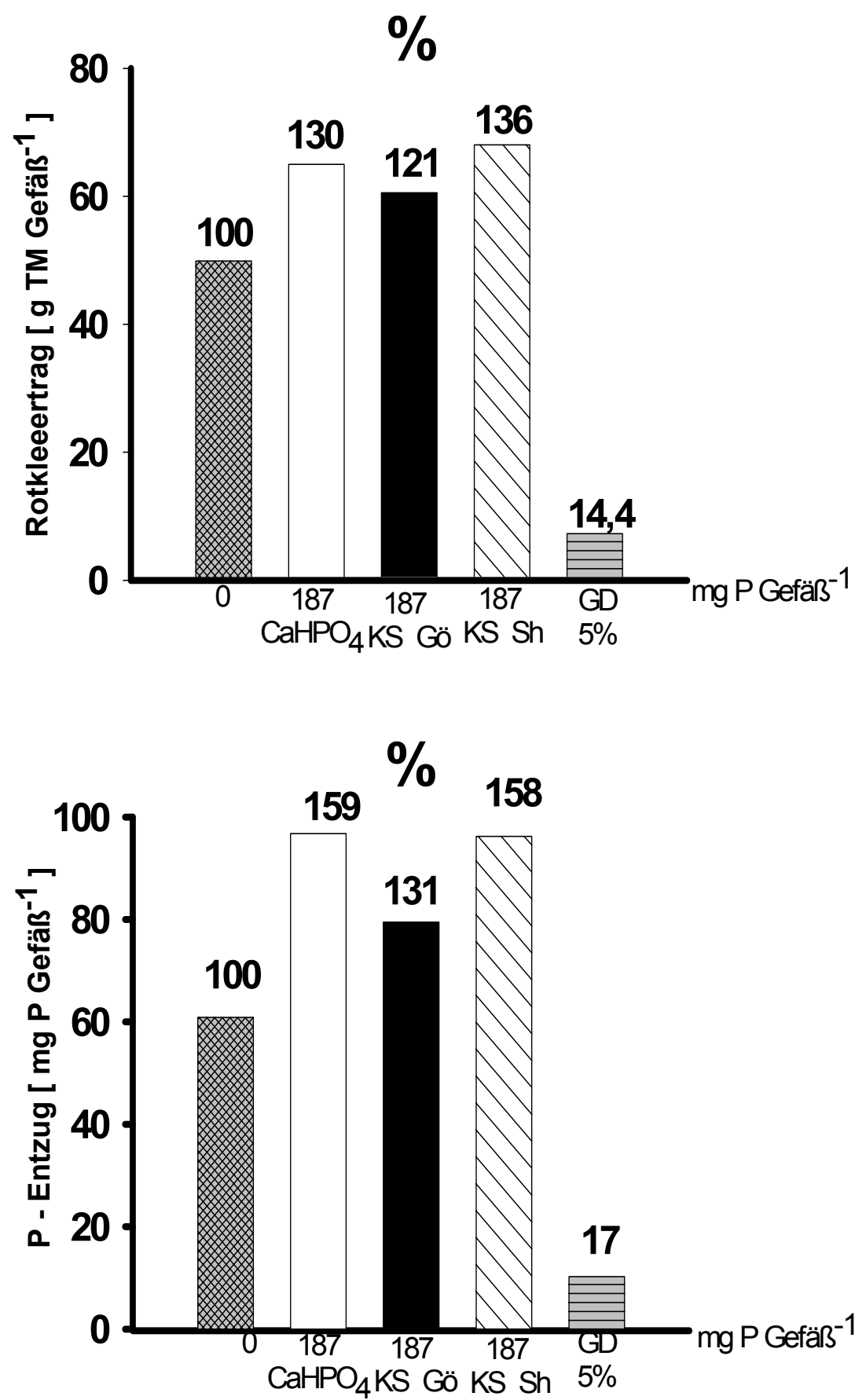

Abbildung 8.5: Abhängigkeit des Rotkleeertrages und des $P$ - Entzuges von der $P$ - Applikation zum Boden Börry P - 0 

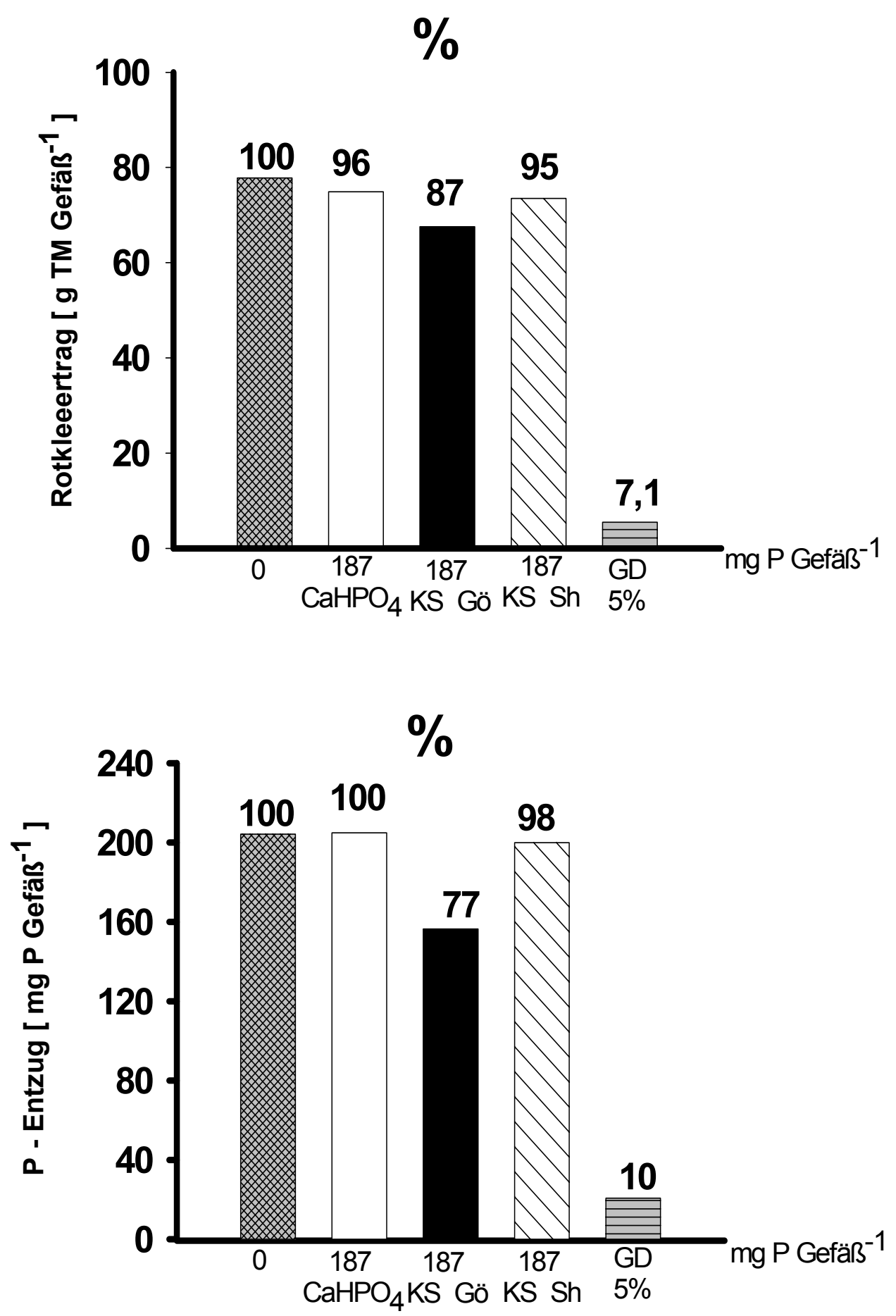

Abbildung 8.6: Abhängigkeit des Rotkleeertrages und des $P$ - Entzuges von der $P$ - Applikation zum Boden Börry P - 80 

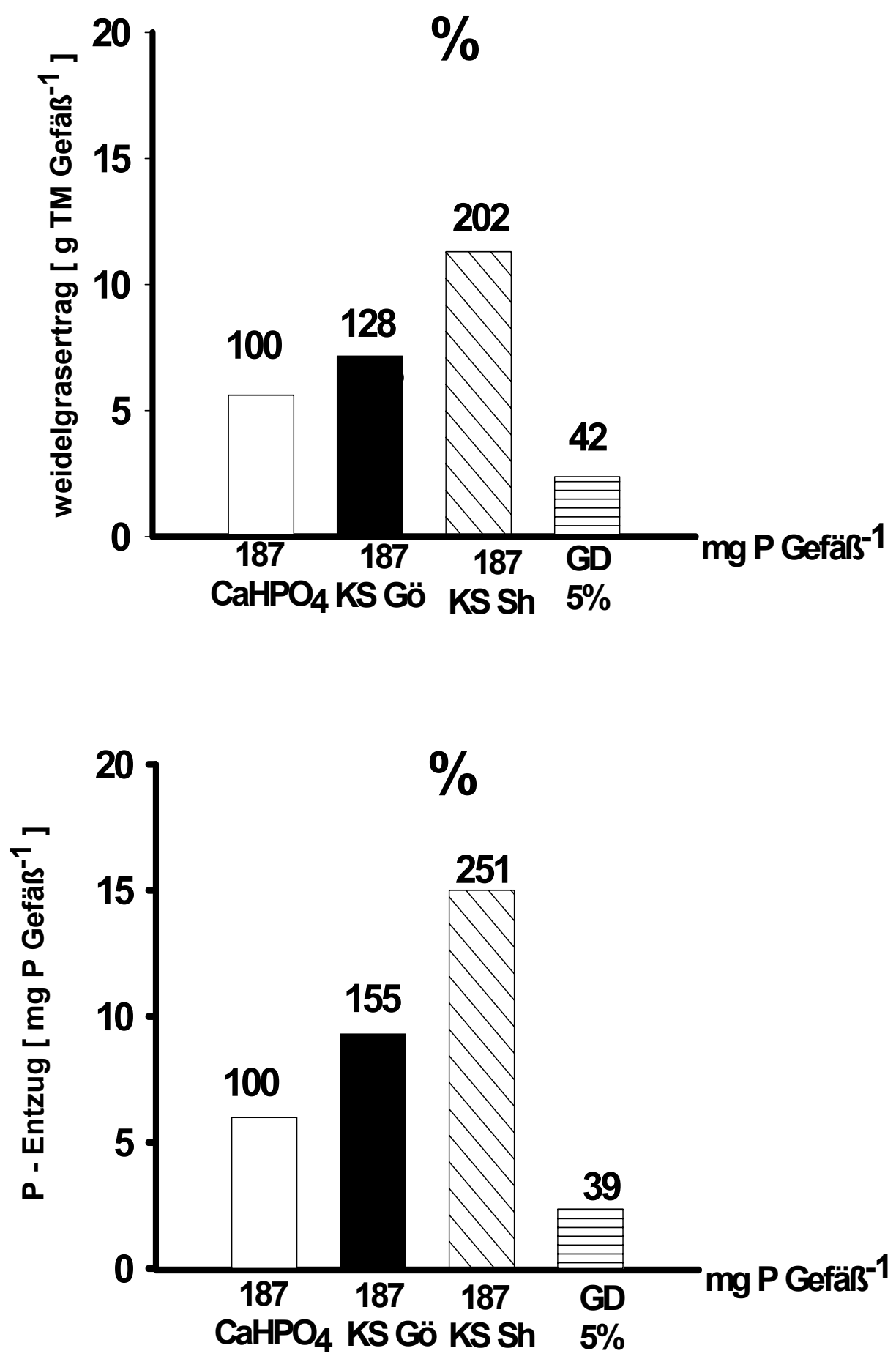

Abb.8. 7: Abhängigkeit des Weidelgrasertrages und des $P$ - Entzuges von der P - Applikation zum Quarzsand 

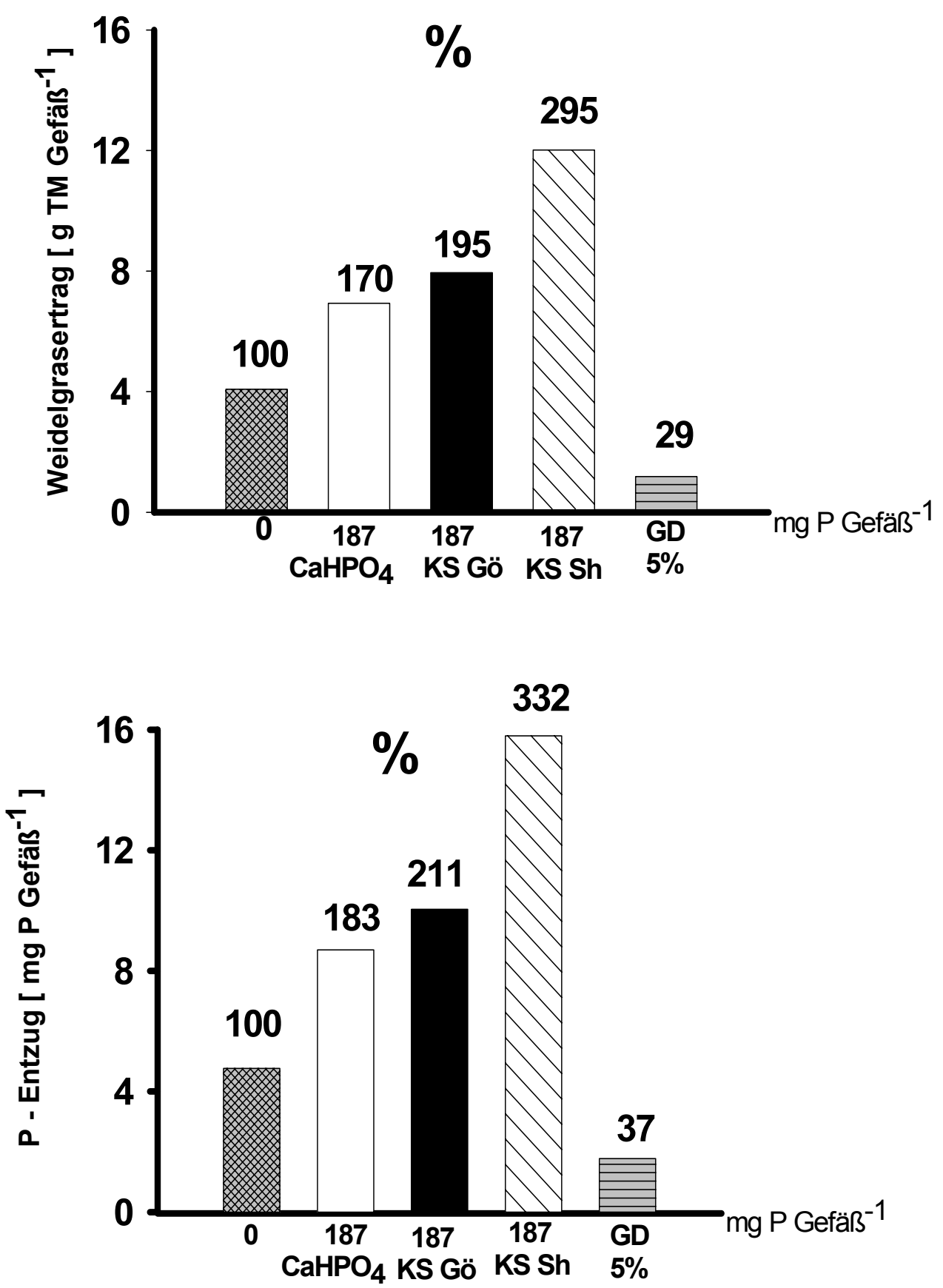

Abbildung 8.8: Abhängigkeit des Weidelgrasertrages und des $P$ - Entzuges von der P - Applikation zum Boden Elliehausen 

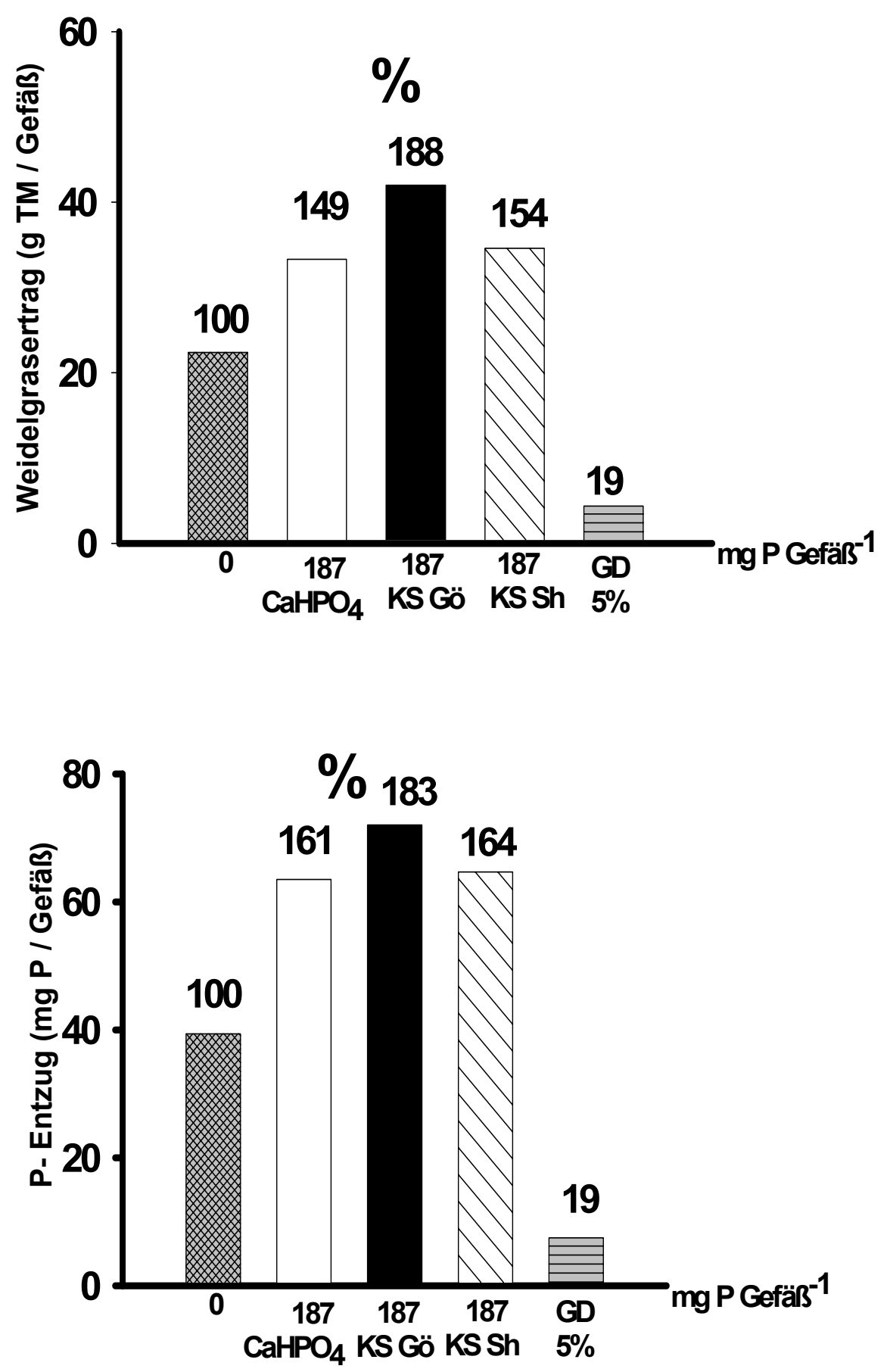

Abbildung 8.9: Abhängigkeit des Weidelgrasertrages und des $P$ - Entzuges von der P - Applikation zum Boden Rehburg - Loccum 

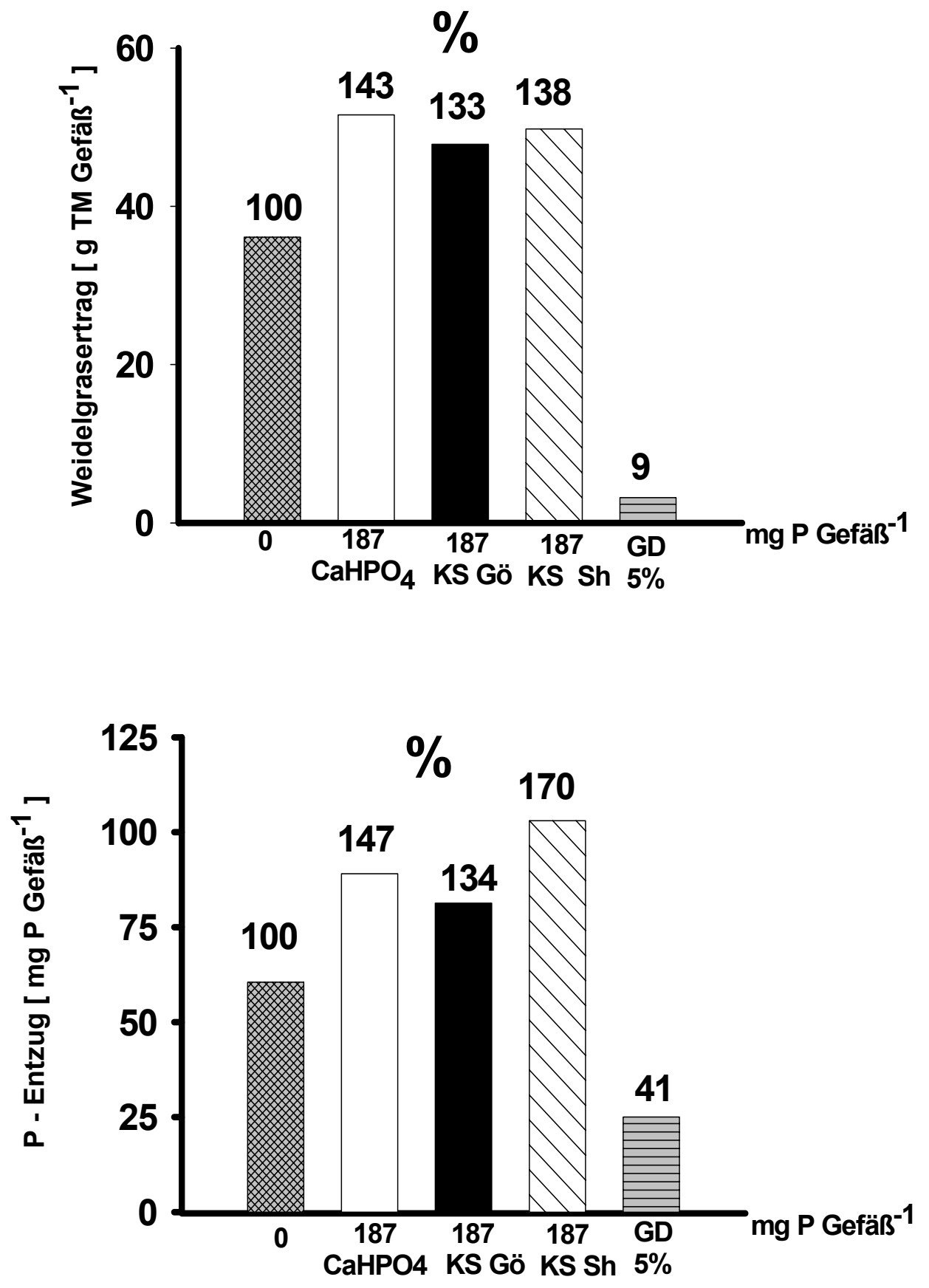

Abbildung 8.10: Abhängigkeit des Weidelgrasertrages und des $P$ - Entzuges von der $P$ - Applikation zum Boden Düshorn (Brache) 

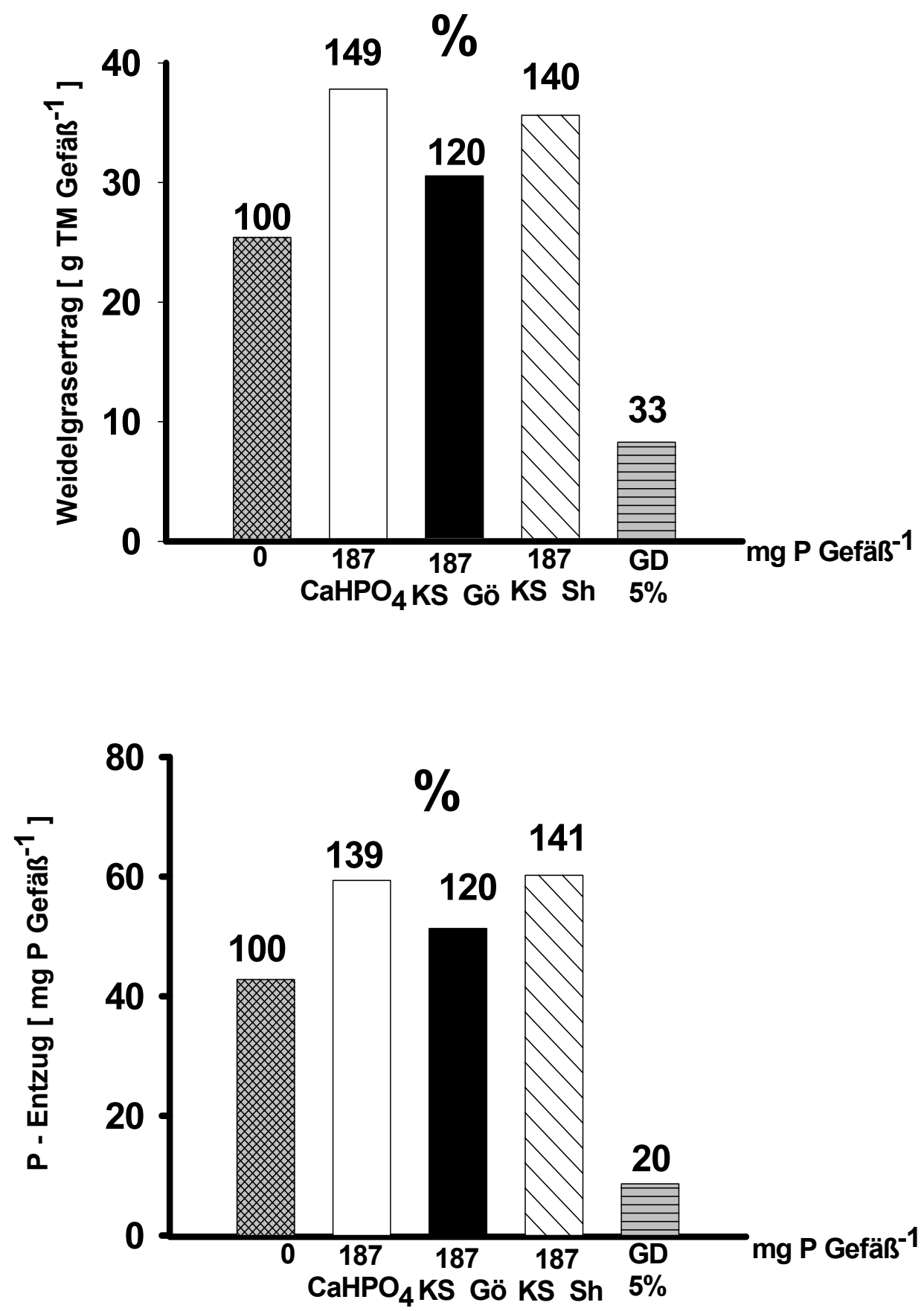

Abbildung 8.11: Abhängigkeit des Weidelgrasertrages und des $P$ - Entzuges von der $P$ - Applikation zum Boden Börry $P$ - 0 

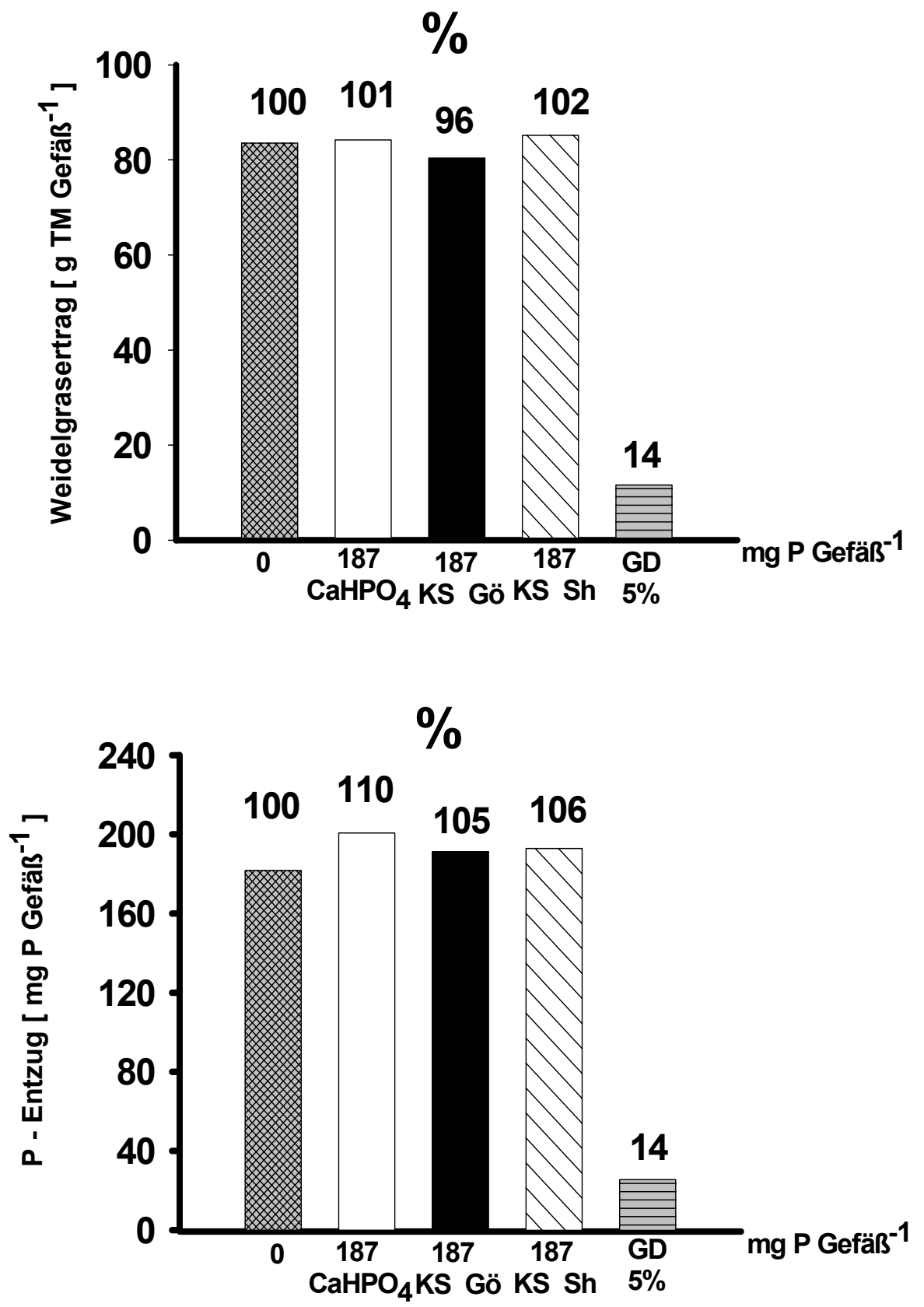

Abbildung 8.12: Abhängigkeit des Weidelgrasertrages und des $P$ - Entzuges von der $P$ - Applikation zum Boden Börry P - 80 


\section{Danksagung}

Mein Dank gilt vor allem Herrn Prof. Dr. W. Römer, der sich als eine nie versiegende Quelle an nützlichen Informationen erwiesen und immer viel Geduld für mich aufgebracht hat. Mein herzlicher Dank gilt dem entgegengebrachten Vertrauen und der Unterstützung bei der Durchführung dieser Arbeit. Ohne seine väterliche Betreuung wäre diese Arbeit erfolglos geblieben.

Ferner danke ich Herrn Prof. Dr. B. Meyer für die Abnahme der mündlichen Prüfung (Februar 2000) und die freundliche Übernahme des Korreferates.

Insbesondere Frau B. Eichenberg und Frau S. Koch sowie Frau M. Niebuhr und den Technikern Herrn E. Schreiber, H. Schröder, J. Kobbe, H.-D. Vohrmann und Herrn R. Hilmer möchte ich für die tatkräftige Mitarbeit bei zahlreichen Versuchen, vom Ansetzen von Gefäßen bis hin zur Durchführung von Analysen und Anfertigung von Postern, ganz herzlich danken.

Unserer Sekretärin Frau M. Noltkämper möchte ich für ihre Hilfe im Laufe meiner Promotion ganz herzlich danken.

Bei allen Professorinnen und Professoren, Mitarbeitern und Kollegen des Institutes für Agrikulturchemie möchte ich mich für die gute Zusammenarbeit in den letzten Jahren ganz herzlich bedanken.

Die Arbeiten wurden durch die Niedersächsische Umweltstiftung und durch das Niedersächsische Ministerium für Ernährung, Landwirtschaft und Forsten gefördert.

Für die finanzielle Unterstützung danke ich der ägyptische Regierung im Laufe meiner Promotion in Deutschland aufrichtig.

Bei allen Professorinnen und Professoren, Kollegen und Mitarbeitern an der Landwirtschaftlichen Fakultät in Moschtohor der Universität Zagazig / Banha, besonders der Abteilung Bodenkunde, möchte ich mich für das entgegengebrachte Vertrauen und die Unterstützung während meines Studiums an der Fakultät bedanken.

Viel mehr als sich mit Worten sagen lässt, gilt letztlich mein herzlicher Dank meiner lieben Frau Hanan Fadlalla und meiner lieben Tochter Jasmine. Sie haben mich mit viel Liebe und Geduld durch alle Motivationstiefs begleitet und mir während meines Forschungsstudiums viel Freude geschenkt. 


\section{Lebenslauf}

\section{Persönliche Daten}

Name:

Vorname:

Geburtsdatum:

Geburtsort:

Familienstand:

Staatsangehörigkeit:
Abd El - Samie

Ihab Mohamed Farid

17.05 .1968

Kairo, Ägypten

Verheiratet, 3 Kinder

Arabische Republik Ägypten

\section{Schulbildung}
$1974-1979$
Grundschule in Kairo, Ägypten
$1979-1982$
Mittelschule in Kairo, Ägypten
$1982-1985$
Gymnasium in Kairo, Abschluß mit Abitur, Kairo, Ägypten

\section{Hochschulausbildung}

$1985-1987$

$1987-1990$

$1991-1995$

$1995-1997$

$1998-2002$
Studium am Institut für Technische Chemie, Kairo, Ägypten

Studium an der Landwirtschaftlichen Fakultät in Moschtohor der Universität Zagazig / Banha, Abschluß: B.Sc. im Fachbereich Bodenkunde

Magister an der Landwirtschaftlichen Fakultät in Moschtohor der Universität Zagazig / Banha, Abschluß: M.Sc. im Fachbereich Bodenkunde und Pflanzenernährung

Doktorand an der Landwirtschaftlichen Fakultät in Moschtohor der Universität Zagazig / Banha

Stipendiat der ägyptischen Regierung und Doktorand mit dem Ziel der Promotion am Institut für Agrikulturchemie der Georg-August-Universität Göttingen

\section{Bisherige Tätigkeiten}

$1990-1994$

$1994-1995$

ab 1995

$1998-2002$
Forschungsstudent an der Landwirtschaftlichen Fakultät in Moschtohor der Universität Zagazig / Banha, in der Abteilung Bodenkunde.

Assistent an der Landwirtschaftlichen Fakultät in Moschtohor der Universität Zagazig / Banha, in der Abteilung Bodenkunde.

Assistentslehrer an der Landwirtschaftlichen Fakultät in Moschtohor der Universität Zagazig / Banha, in der Abteilung Bodenkunde.

Doktorand am Institut für Agrikulturchemie der Georg-August-Universität Göttingen 


\section{PUBLIKATIONEN}

\section{Geleistete Öffentlichkeitsarbeit}

1. W. Römer und I. F. Samie (2000): „Einfluss eisenhaltiger Klärschlämme auf Kenngrößen der P-Verfügbarkeit von Lehm- und Sandböden“. 112. VDLUFAKongress in Stuttgart-Hohenheim, 2000, 219-226.

2. Wilhelm Römer und Ihab F. Samie (2001): Einfluss eisenhaltiger Klärschlämme auf Kenngrößen der P-Verfügbarkeit in Ackerböden Zeitschrift „Bodenkunde und Pflanzenernährung. 164, 321-328.

3. Ihab F. Samie und Wilhelm Römer (2001): Poster „Phosphorus availability to maize plants from sewage sludge treated with Fe compounds" auf dem Greidinger Symposium in Lissabon, organisiert von 'The International Fertiliser Society', März 2001, 531- 534.

4. Ihab F. Samie und Wilhelm Römer (2001):Poster „Phosphorus availability to maize plants from sewage sludge treated with Fe compounds" auf dem XIV. International Plant Nutrition Colloquium in Hannover, 2001 (vgl.; In :W. J. Horst et al.: Plant Nutrition - Food security and sustainability of agro-ecosystems, 846-847, 2001).

5. Vortrag auf der Tagung am 3. International Symposium - cum - Workshop in Kairo zum Thema „Phosphorus availability to maize plants from sewage sludge treated with Fe compounds“.Kairo, Ägypten im Sept. 2001.

6. Wilhelm Römer und Ihab F. Samie (2002): „Phosphordüngewirkung eisenhaltiger Klärschlämme“ Zeitschrift „Bodenkunde und Pflanzenernährung. $165,83-91$.

7. Wilhelm Römer und Ihab F. Samie (2002): „Eisenreiche Klärschlämme sind für das P-Recycling im Ackerbau ungeeignet“ Zeitschrift „Wasser und Boden. 54/6, 28-32.

8. Wilhelm Römer, Ihab F. Samie, Matthias Neubert und Detlef Merkel (2003): „PDüngewirkung von 13 Klärschlämmen mit unterschiedlichen Eisengehalten." KA Wasserwirtschaft, Abwasser, Abfall; angenommen Dez. 2002.

9. W. Römer, I. F. Samie, M. Neubert und D. Merkel (2002): Poster „PDüngewirkung von 13 eisenhaltigen Klärschlämmen“ 114. VDLUFA-Kongress in Leipzig, (Sept. 2002), Text auf CD. 


\section{Three Abstracts}

Influence of iron content in sewage sludges on parameters of phosphate availability in arable soils

Wilhelm Römer und Ihab F. Samie

(J. Plant Nutr. Soil Sci. (2001), 164, 321-328)

\section{Abstract}

The use of iron salts for the $\mathrm{P}$ elimination in purification plants is widely used. But it is not clear whether the $\mathrm{P}$ availability in arable soils is negatively influenced by iron compounds or not. Therefore the aim of the investigations was to study the influence of two sewage sludges with a high and a low Fe content respectively on $\mathrm{P}$ sorption and phosphate concentration $\left(\mathrm{P}_{\mathrm{i}}\right)$ in the soil solution after application of $\mathrm{CaHPO}_{4}$ or sewage sludge to 5 loamy and 4 sandy soils (pot experiments and 1 silty loam (field experiment)). Soils were analysed 1, 6 and 13 months after $P$ application. Sludge Gö contained $12 \mathrm{~kg} \mathrm{P}$ and $65 \mathrm{~kg}$ $\mathrm{Fe} \mathrm{t}^{-1} \mathrm{DM}(\mathrm{P}: \mathrm{Fe}=1: 5,4)$ and sludge Sh $25 \mathrm{~kg} \mathrm{P}$ and $39 \mathrm{~kg} \mathrm{Fe} \mathrm{t}^{-1} \mathrm{DM}(\mathrm{P}: \mathrm{Fe}=1: 1,5)$. The basic $\mathrm{P}$ application was $60 \mathrm{~kg} \mathrm{P} \mathrm{ha}^{-1}$ (= $30 \mathrm{mg} \mathrm{P} \mathrm{kg}^{-1}$ soil in the pot experiment, as sludge or as $\mathrm{CaHPO}_{4}$ ). $\mathrm{P}$ uptake by maize was determined in a separate pot experiment with a loamy soil and the same $\mathrm{P}$ application rate.

The $\mathrm{P}$ sorption capacity remained similar in all soils after application of sludge $\mathrm{Sh}(\mathrm{P}: \mathrm{Fe}=$ $1: 1,5)$ compared with soils without sludge, however, after application of sludge Gö the $P$ sorption increased to $16 \%$ (0-59\%). After application of sludge Sh the mean $\mathrm{P}_{\mathrm{i}}$ concentration increased in loamy soils to $34 \%$ and in sandy soils to $15 \%$. On the other hand the $\mathrm{P}_{\mathrm{i}}$ concentration decreased after applying sludge Gö to $13 \%$ and $36 \%$ as compared to the controls of the respective soils. In the field experiment the $P_{i}$ concentration of plots with a high $\mathrm{P}$ level $\left(50 \mathrm{mg}\right.$ lactate soluble $\mathrm{P} \mathrm{kg}^{-1}$ soil) was also significantly decreased after application of $10 \mathrm{t}$ sludge $\mathrm{Gö} \mathrm{(126} \mathrm{kg} \mathrm{P} \mathrm{ha-1)} \mathrm{in} \mathrm{comparison}$ with triple phosphate. One month after the application of increasing amounts of sludge Gö $\left(5,10,15 \mathrm{t} \mathrm{DM} \mathrm{ha}^{-1}\right)$ both the concentration of oxalate-soluble $\mathrm{Fe}$ in the soil and the $\mathrm{P}$ sorption were increased. The elevated relationship between these two parameters was highly significant $\left(r^{2}=0.6-0.97\right)$. Plant uptake of $P$ was less after application of sludge $G o ̈$ than after application of sludge $\mathrm{Sh}$ and much less than $\mathrm{P}$ uptake from $\mathrm{CaHPO}_{4}$. Sewage sludges with a P : Fe ratio of $1: 5$ should not be recommended for agricultural use, as the $P$ availability is significantly reduced. Iron salts should not be used for conditioning of sludges. 


\section{Phosphorus fertilization effects of sewage sludges treated with iron compounds.}

Wilhelm Römer und Ihab F. Samie

(J. Plant Nutr. Soil Sci. (2002), 165, 83-91)

\section{Abstract}

The result of waste water treatment with iron salts are sewage sludges with higher $\mathrm{P}$ but also Fe contents. The effect of such sludges on $\mathrm{P}$ availability in soils is not clear and was, therefore, compared with $\mathrm{CaHPO}_{4}$ as a $\mathrm{P}$ mineral fertilizer. In experiments (Mitscherlich pots, $6 \mathrm{~kg}$ soil) two sandy soils ( $\mathrm{pH} 4.3$ and 4.9), two luvisols ( $\mathrm{pH} 6.3$ and 7.2), a loess $\left(13 \% \mathrm{CaCO}_{3}\right)$ and quartz sand all differing in their $\mathrm{P}$ status were treated with two sewage sludges which differed in their molar Fe:P ratios (sludge Gö: 1:0.3, sludge Sh: 1:1.2). For sludge $\mathrm{Gö}$ the $\mathrm{P}$ elimination had been carried out with $\mathrm{FeSO}_{4}$ and the sludge was stabilized with $\mathrm{FeCl}_{3}+\mathrm{Ca}(\mathrm{OH})_{2}$ (filter press). For sludge Sh $\mathrm{P}$ was eliminated by $\mathrm{FeCl}_{3}$ in the aerobic basin.

The first crop was maize (total shoot dry matter), the second red clover. In case of sludge Sh both crops took up in comparison to $\mathrm{CaHPO}_{4}$ the same amount of $\mathrm{P}$ from all substrates with the exception of quartz sand. The effect of sludge Gö was quite different: from the neutral luvisol soils maize was able to take up only 64-82 \% $\mathrm{P}$ and red clover $77-82 \% \mathrm{P}$ only as compared to $\mathrm{CaHPO}_{4}$. On the sandy soils maize and red clover grew after sludge Gö as good or better than after $\mathrm{CaHPO}_{4}$ application and $\mathrm{P}$ uptake was quite similar (83$106 \%$ ). This result was concomitant to an increase of $\mathrm{pH}$ values (from 4.3 to 4.9, 4.9 to 5.6 , respectively). The negative efficiency of $P$ uptake in heavy soils after sludge $G o ̈$ application resulted from an increase of $\mathrm{P}$ sorption and decrease of orthophosphate concentration in soil solution. This sludge contains high proportion of iron hydroxides / oxides with free sorption sites for $P$. In future $P$ elimination from waste water should be done without iron salts. At least during the process of conditioning no Fe salts should be used. 


\section{Phosphate fertilization effects of 13 sewage sludges from purification plants with P precipitation by iron salts}

W. Römer, I.F. Samie, M. Neubert, D. Merkel

(KA, Wasserwirtschaft, Abwasser, Abfall, accepted for printing, 2003)

\section{Abstract}

In Germany, 500.000 t of iron salts are annually used to precipitate $\mathrm{P}$ in purification plants. The sewage sludge (SS) is used as a fertilizer. However, it can be assumed that the iron compounds of the SS may negatively influence the $\mathrm{P}$ availability in soils. Therefore, the $\mathrm{P}$ fertilization effect of 13 SS with different Fe : P ratios (from $7.4: 1$ to $0.2: 1$ ) was tested in comparison to triple superphosphate (TSP) in an experiment with rye grass grown on a humic sandy soil moderate in P supply. TSP increased P removal by plants to $149 \%$ of the unfertilized control, whereas the removal in the SS treatments ranged from 107 to $149 \%$ (average 136\%). Phosphate uptake of the grass was negatively correlated with $\mathrm{P}$ sorption in the soil $\left(r=-0.57^{*}\right)$ and oxalate soluble Fe of soil $\left(r=-0.66^{* *}\right)$, but positively correlated with orthophosphate concentration in soil solution $\left(r=0.82^{* * *}\right)$. The $P$ uptake was also negatively correlated with the Fe : $\mathrm{P}$ ratio of the SS $\left(r=-0.93^{* * *}\right)$ and was significantly lower than in the TSP treatment at $\mathrm{Fe}: \mathrm{P}$ ratios higher than $1.4: 1$. These SS are not recommendable as $\mathrm{P}$ fertilizer. 